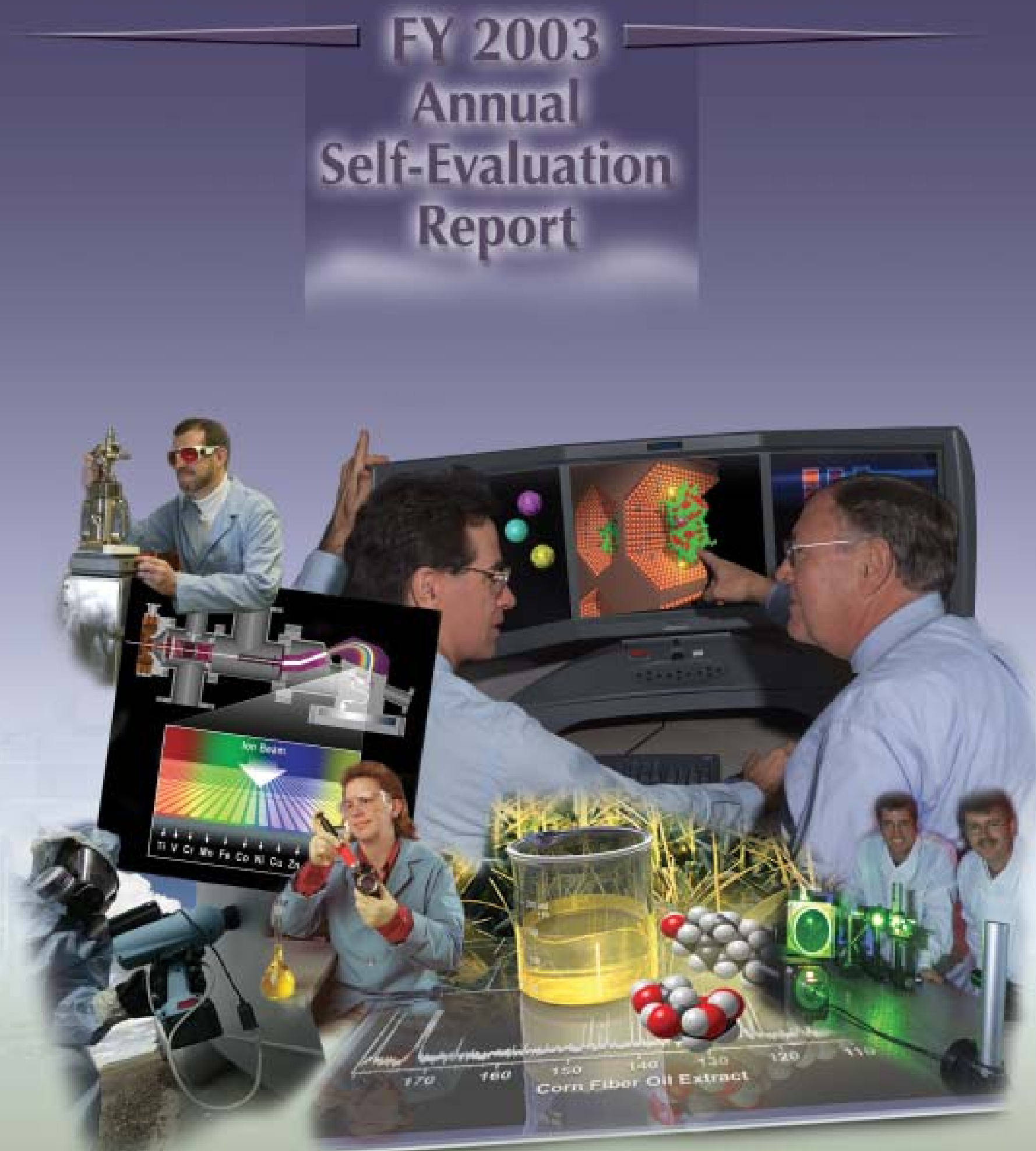

Pacific Northwest

National Laboratory

Operated by Battelle for the

U.S. Department of Energy 


\title{
DISCI.ATMER
}

This report was prepared as an sccount of wotk sponsored by an agency of the United Seases Government.

Reference herein to any specific comenefcial prodact, process, of service by trade name, trademark,

manufacturer, or otherwise does not necessarily constitute of imply its endorsement, recommendation, or favoring, by the United States Government ot any apency theneof, or Battelle Memotial Institute.

\author{
PACIFIC NORTHWEST NATIONAL LABORATORY \\ operated by \\ BATTEI.E \\ for the \\ UNTTED STATES DEPARTMFNT OF ENERGY \\ under Coninct DE-AO06-76RL01830
}

Printed in the United States of Ameriea

Avwilable to DOE and DOF contractors from the

Office of Scientific and Technical Information, P.O. Bax 62, Oak Ridge, TN 378.31; prices svalabie from (615) 576-8401.

Avalibicic to the public from the National Technical Information Service, US. Department of Commerec, S285 Port Royal Rd., Springfield, VA 22161

PNNL - 14421

\section{On the cover...}

2 The Al tie Sigral, Na the Tine ishatiocal place amap datecior novilutueizes iont catection in mrss spectrometry instrumets. What thes

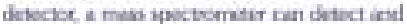
measue a brosd arye of kon mases form a

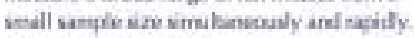
Whik iraitional mins gectireneyes provide a kevhole view of a witall postion of the sectrum, the Asat driector opern the

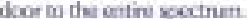

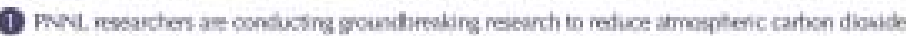

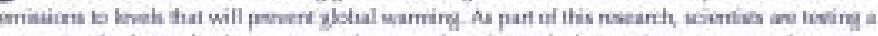

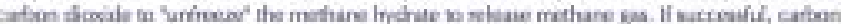

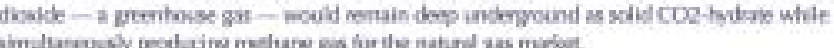

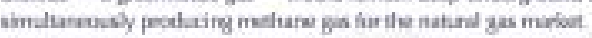

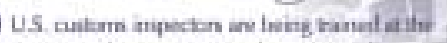
Departnerk of Enegr' ' Hazanbis Maieriah

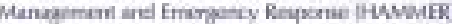
Traning Center on the Haniond ste bo ase acount:

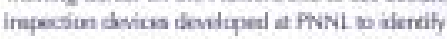
wespons and esplonien. This colahoratican hetss It imale fumainal secinity a liele figlat

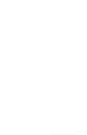




\section{FY2003 Annual Self-Evaluation Report for the Pacific Northwest National Laboratory}

October 2003

This document directly supports the DOE-RL Contract for the Management and Operation of the Pacific Northwest National Laboratory Contract DE-AC06-76RL01830, Appendix E. 


\subsection{Purpose and Format of this Report}

\section{This FY2003 Annual Self-Evaluation Report, a deliverable under Contract DE-AC06-76RL01830, satisfies the requirements of PNNL's Performance Evaluation and Fee Agreement and DOE Order 224.1. This year's document continues the modular design format initiated in FY2002 and responds to DOE comments and suggestions for even further clarity and coherence.}

\section{Purpose of this Self-Evaluation Report}

This year's FY2003 Self-Evaluation Report presents a focused, quantitative, and objective approach to evaluating the performance of the Laboratory. Its purpose is to summarize Pacific Northwest National Laboratory's (PNNL) progress toward accomplishment of the Critical Outcomes, objectives, and performance indicators that were developed in partnership with the U.S. Department of Energy (DOE) and codified in the FY2003 Performance Evaluation and Fee Agreement (PEFA). In addition, this report summarizes the adequacy of PNNL's integrated assessment processes and PNNL's strengths and opportunities for improvement, and provides a history of PNNL accomplishments and awards.

This report consists of the following sections

Executive Summary-This section provides an overall performance summary and individual performance summaries for each of the three Critical Outcomes.

Part I - Status of Performance Against Critical Outcomes-This part of the report presents the results and analysis of progress made against the key outcomes and expectations. Modules for Objectives $1.1-1.6$ are grouped by mission area; their relation to quality, relevance, research facilities, research program management, leading-edge scientific capabilities, or EMSL user program is shown in footers. All other module numbers correlate to those in the FY2003 PEFA.

Part II - Strengths and Opportunities for Improvement-This portion of the report presents PNNL's analysis of its strengths and opportunities for improvement and, using our strategy mapping efforts this year, identifies the actions most critical to achieving our vision and outcomes.
Part III - Adequacy of PNNL's Integrated Assessment Processes-This portion of the report describes the adequacy of PNNL's integrated assessment processes to establish compliance with key internal controls.

Appendix A shows the adjectival and value ratings for all of the FY2003 Critical Outcomes, objectives and performance indicators

Appendix B shows a history of PNNL's key accomplishments.

Appendix C shows a history of PNNL's significant awards.

Appendix D contains a list of all acronyms listed in this report.

\section{Format of this Report}

The format of this report continues the well-received twopage modular design started last year. In response to DOE suggestions, PNNL has improved the clarity and readability of the modules under Critical Outcome 1.0, Scientific and Technological Excellence, in three ways.

- First, each module in Sections 1.1 through 1.4 is explicitly tied to one of the four performance criteria - quality, relevance, research facilities, or research program management.

- Second, modules that belong together thematically are placed together physically, even though their numbering would suggest otherwise. For example, the modules that addresses 1.5 and 1.6 of the PEFA come along with, not after, the modules in Sections 1.1-1.4 to which they relate.

- Third, each major section in Part I: Status of Performance Against Critical Outcomes, is prefaced by a module summarizing that section in terms of quality, relevance, facilities (if applicable), and management. 


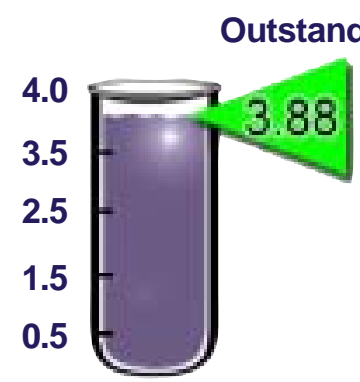

Measurement tube indicating adjectival and numerical rating
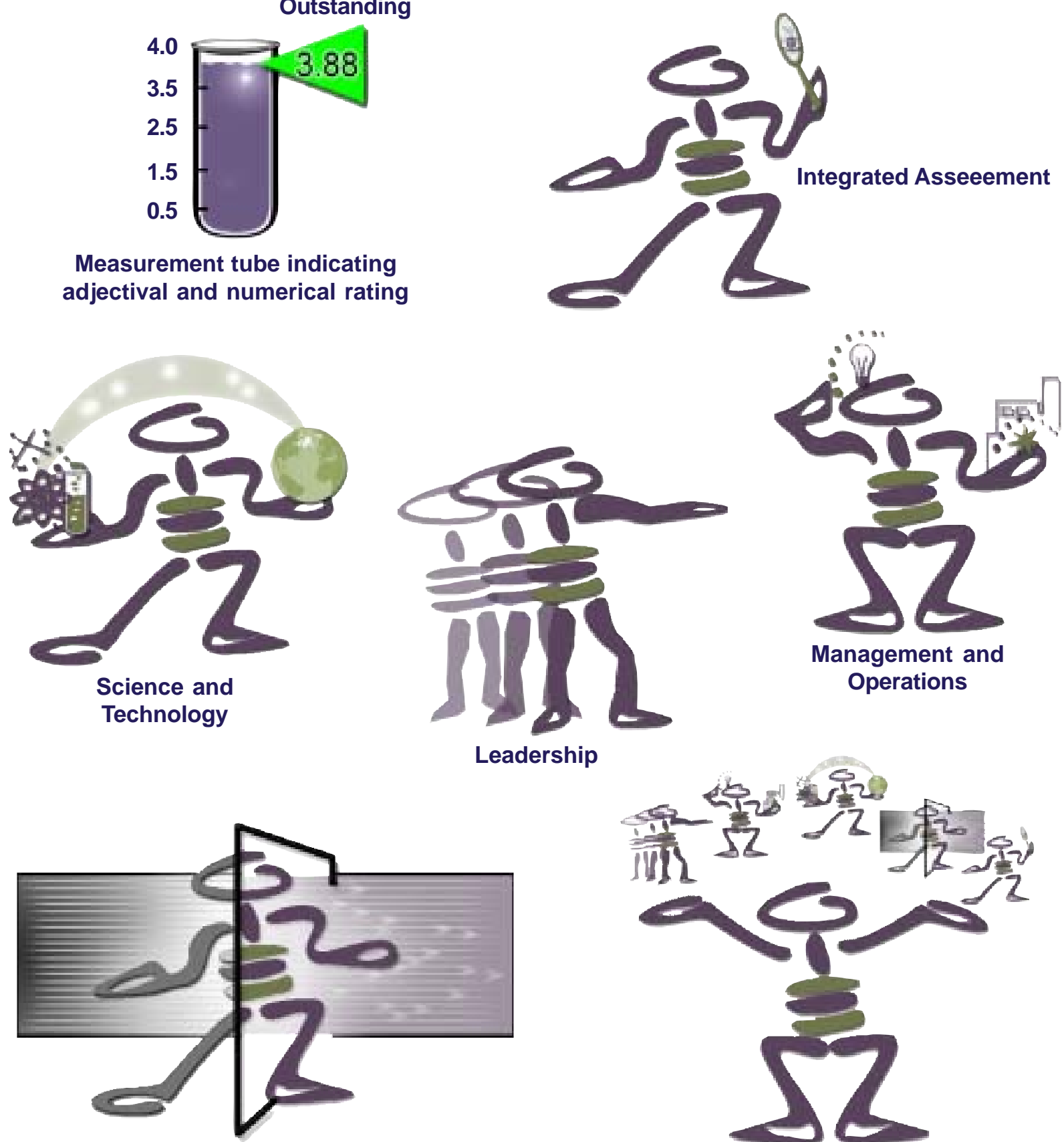

Strengths and Opportunities for Improvements

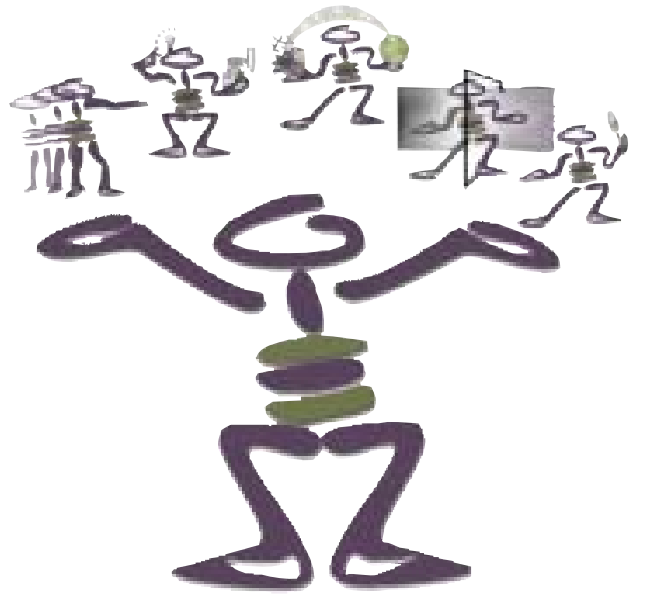

Executive Summary

Figure $\mathbf{0 . 0}$. Icons key the reader to the report's content areas. 

Executive Summary

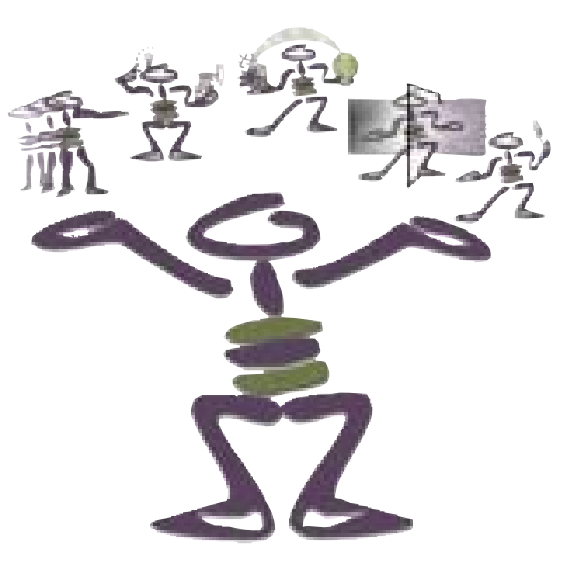


In FY2003, Battelle met or exceeded nearly all U.S. Department of Energy (DOE) expectations in Scientific and Technological Excellence, Management and Operations Excellence, Leadership Excellence, and other specified areas. Based on the results of this self-evaluation, we calculated an overall performance score of 3.9 out of a possible 4.0 points, which translates to an adjectival rating of "Outstanding."

\section{Critical Outcome Performance}

Our assessment of the Laboratory's performance against our critical outcomes is "outstanding." The basis for this assessment is provided in Part I of this report and the scoring details are provided in Appendix A. This does not mean that there is no room for improvement. Part II of this report describes the strengths and weaknesses of our management approach and, in the context of our new contract with DOE, describe important steps for improvement.

\section{Scientific and Technological Excellence}

The Laboratory's science and technology programs are designed to address critical DOE mission needs and provide new insights and solutions to challenges facing the nation and the world. Progress in our science programs during FY2003 supported Office of Science goals and led to significant advances in the fields of chemistry, biology, and computing among others. These advances have beneficial application to all four DOE missions. We delivered significant program accomplishments in the applied mission areas of national security, environmental quality, and energy. External peer reviews confirmed we have achieved national and international recognition in research, the breadth of our research programs, and the high caliber of our staff and facilities.

Our external peer reviewers validated the quality, relevance, and future directions of PNNL's science and technology development. Overall, our programmatic work was considered highly relevant to the national research agenda and in some areas are judged to be world-class.

The Laboratory received important external recognition in FY2003, including three R\&D 100 and three FLC awards. The quality of our scientific efforts are shows in the long list of staff recognized for scientific and engi- neering excellence through awards, invited talks, and participation on scientific committees. In addition, our publication rate is higher than ever, with eight of our top 10 publication venues being among the top ten journals in their respective fields.

\section{Management and Operational Excellence}

Battelle continue to manage and operate the Laboratory with distinction, and is becoming widely known as the DOE benchmark for Laboratory management, stewardship of DOE's assets, and protecting the health and safety of workers, the public, and the environment. Overall, Laboratory operations are exemplary, including strength in Environmental Safety and Health (ES\&H) management, business management, integrated safeguards and security, facility maintenance, and energy conservation. The Laboratory has maintained and enhanced its capabilities and physical infrastructure to meet current and future mission needs, and it developed new tools to integrate management systems for effective, efficient business performance.

\section{Leadership Excellence}

Battelle's leadership in research and development (R\&D) and regional partnerships helps ensure PNNL's stature as an enduring national asset. Our programs are helping establish a robust, sustainable regional economy, and serve to attract, develop, and retain critical staff necessary to achieve our mission, and establish community trust. We did this by identifying "best in class" workgroups, demonstrating the Laboratory's relevance to the economic and Science and Technology (S\&T) needs of the region, enhancing the Laboratory's ability to generate revenues from commercialization, and enhancing leadership and diversity in science and engineering education through Lab-sponsored programs for students and educators.

FY2003 PNNL Annual Self-Evaluation Report 
And more. ...

In addition to these three critical outcomes, Battelle is committed to continual improvement and a strong integrated assessment process. Although these management functions are not scored per se in the performance rating, they underpin and reinforce our ability to achieve outstanding performance in the critical outcomes.

\section{Strengths and Opportunities for Improvement}

Through our strategy mapping efforts this past year, we have more clearly focused and articulated our vision, outcomes, and strategy. Our strategy captures the actions critical to achieving our vision and outcomes actions that capitalize on the strengths and opportunities for improvement that will leverage those laboratory systems, processes, and competencies most essential to realizing our vision and outcomes. We sustained the strengths we had before, we've made significant progress on all the improvements we said we needed to, and we see further opportunities to improve how we deliver important, high-quality science-based solutions.

For more information, please refer to Part II of this report.

\section{Adequacy of PNNL's Integrated Assessment Process}

PNNL's robust process for promulgating appropriate contractual and regulatory requirements and assessing performance against them forms a strong foundation to meet DOE expectations for corporate assurance. This process will be further improved during FY2004 by systematizing internal controls for assurance at the Battelle-
Corporate level. We look forward to working with DOE and the PNSO to realize the intention of the new contract in this regard.

For more information, please refer to Part III of this report.

\section{Partnering for Success}

The Laboratory's success hinges on a strong partnership with DOE. Noteworthy examples include:

- A new contract extending our relationship into 2007, with partnering a major theme. Outstanding performance will mean higher fees; for the first time, Mission Stretch Goals have been added to further incentivize great science. In return, DOE will hold Battelle to a higher standard of accountability for meeting the Government's requirements and expectations. Finally, mutual trust will be the critical component when Battelle uses the contract's flexibility to propose alternative standards that provide greater effectiveness and efficiency while still meeting DOE's needs.

- Development of a plan for our orderly transition of the Radiological Processing Laboratory

- Design of a new fee agreement that reflects the intentions and guidelines of the new contract

- Helped resolve contractual issues regarding the 900 MHz NMR

- Development of an evolving plan for transmitting critical capabilities in the 300 area while establishing the capacity for modernizing and enhancing overall Lab capabilities that are important to DOE and the future of this Laboratory.

Table 0.1. The value points and associated adjectival ratings PNNL believes are justified based on our performance lead to an overall FY2003 self-rating of "Outstanding." Details for all FY2003 Critical Outcomes, objectives, and performance indicators are provided in Appendix A.

\begin{tabular}{|l|c|c|c|c|}
\hline Critical Outcome & $\begin{array}{c}\text { Value } \\
\text { Points }\end{array}$ & $\begin{array}{c}\text { Adjectival } \\
\text { Rating }\end{array}$ & Weight & $\begin{array}{c}\text { Weighted } \\
\text { Score }\end{array}$ \\
\hline $\begin{array}{l}\text { 1.0 } \\
\text { Scientific and Technological } \\
\text { Excellence }\end{array}$ & 3.98 & Outstanding & $60 \%$ & 2.39 \\
$\begin{array}{l}\text { Management and Operations } \\
\text { Excellence }\end{array}$ & 3.88 & Outstanding & $25 \%$ & 0.97 \\
3.0 & 3.70 & Outstanding & $15 \%$ & 0.56 \\
\hline
\end{tabular}




\section{Overall Self-Evaluation of Critical Outcome 1.0, Scientific and Technological Excellence}

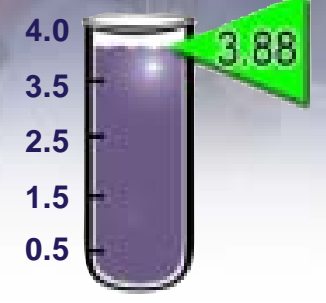

PNNL conducts high-quality, leading-edge scientific and technological research and development programs that are recognized by the scientific community. The Laboratory creates scientific capabilities and user programs that support critical DOE mission needs and address other national priorities. In FY2003, PNNL met or exceeded nearly all DOE expectations in Scientific and Technological Excellence.

Battelle's evaluation of its performance under Critical Outcome 1.0, Scientific and Technological Excellence, indicates a score of 3.98 out of 4.0, for a rating of Outstanding. Details of the evaluation are provided in Part I, Section 1 of this report. Adjectival and value ratings for Critical Outcome 1.0 and its supporting objectives and performance indicators are summarized in Appendix A, Tables A.3 - A.7.

\section{Highlights of the Six Performance Objectives}

\section{1/1.2 Quality of Science and Technology and Relevance to DOE Mission and National Needs}

Our FY2003 peer reviews and customer results indicate that PNNL's scientific and technical output meet or exceeded expectations across all our mission areas. Accomplishments related to this objective reside within the modules in sections 1.1, 1.2, 1.3, and 1.4, and are specifically called out in page footers.

\subsection{Success in Constructing and Operating Research Facilities}

In FY2003, PNNL made important progress towards increasing the effectiveness and efficiency of its user access program. Accomplishments related to this objective reside in modules 1.1.6 and 1.1.7.

\subsection{Effectiveness and Efficiency of Research Program Management}

PNNL continues to do an outstanding job of delivering science and technology that contributes to our customers goals and objectives in an efficient and effective manner. We deliver products on time while meeting customer expectations, and we team effectively with our customers to develop plans and alternatives for using science and technology to meet their needs. Accomplishments related to this objective reside within the modules in sections 1.1,1.2,1.3, and 1.4, and are specifically called out in page footers.

1.5 Create Leading-Edge Scientific Capabilities To Support Evolving DOE Mission Needs

$\checkmark$ 1.5.1.1 Goal: BSI recruiting; targeted offers for new hire are met for key staff. Result: PNNL exceeded the number of target hires. (Module 1.1.10)

$\checkmark$ 1.5.1.2 Goal: BSI partnerships; $\$ 60 \mathrm{M}$ in high quality proposals submitted to DOE and NIH. Result: PNNL exceeded its outstanding target, achieving $\$ 72 \mathrm{M}$ in proposals. (Module 1.1.10)

$\checkmark$ 1.5.1.3 Goal: BSI technical achievements; complete milestones for three LDRD projects. Result: All three projects completed. (Module 1.1.10)

$\checkmark$ 1.5.1.4 Goal: BSI S\&T progress; 50 or more articles submitted for publication in peer-reviewed journals. Result: More than 60 papers have been submitted for publication. (Module 1.1.10)

$\checkmark$ 1.5.1.5 Goal: Feedback from BSI External Advisory/Review Committee. Result: Committee feedback was positive. (Module 1.1.10)

- 1.5.2.1 Goal: Complete 5 or more of CS\&EI science and technology goals. Result: All 6 technical research goals completed. (Module 1.1.11) 
1.5.2.2 Goal: CS\&E visibility activities; submission of papers, host seminars, organize research exhibit, and up date CS\&E website. Result: All activities either fully met or exceeded expectations. (Module 1.1.11)

$\checkmark$ 1.5.2.3 Goal: Feedback from CS\&EI Advisory/Committee. Result: Committee feedback was positive. (Module 1.1.11)

$\checkmark$ 1.5.3.1 Goal: NSTI visibility activities; submit 11 new papers, present 10 technical papers, host 5 seminars, and overhaul NSTI website. Result: All activities completed. (Module 1.1.12)

$凶$ 1.5.3.2 Goal: NSTI program development; prepare three proposals, reconvene outside steering committee, and hire two staff. Result: Four proposals prepared, committee reconvened but only one hire was made. (Module 1.1.12)
\ 1.5.3.3 Goal: NSTI scientific impact; 4 scientific goals achieved. Result: Three scientific goals achieved. (Module 1.1.12)

\subsection{Improve Scientific Impact of EMSL User Program}

$凶$ 1.6.1 Goal: Develop and issue a plan; establish a steering committee; facilitate a workshop; and provide input for call for grand challenges proposals. Result: Plan issued; steering committee established; BGGC workshop postponed until November 2003; and calls delayed until BGGC and BGC workshops completed. (Module 1.1.6)

- 1.6.2 Goal: Hold a workshop; develop an EMSL Operations Manual; implement the new model. Result: Workshop was held; manual was developed; and model is now operational. (Module 1.1.7)

Table 0.2. PNNL's overall self-evaluation of performance under Critical Outcome 1.0 indicates a score of 3.98 out of 4.0 , for a rating of "Outstanding."

\begin{tabular}{|c|c|c|c|c|}
\hline Element & $\begin{array}{l}\text { Total } \\
\text { Points }\end{array}$ & Adjectival Rating & $\begin{array}{l}\text { Objective } \\
\text { Weight }\end{array}$ & $\begin{array}{l}\text { Total } \\
\text { Points }\end{array}$ \\
\hline $\begin{array}{l}\text { 1.1 Quality of Science and } \\
\text { Technology; } \\
\text { 1.2 Relevance to DOE Mission } \\
\text { and National Needs; } \\
\text { 1.3 Success in Constructing } \\
\text { and Operating Research } \\
\text { Facilities } \\
\text { 1.4 Effectiveness and } \\
\text { Efficiency of Research } \\
\text { Program Management }\end{array}$ & 4.00 & Outstanding & $80 \%$ & 3.20 \\
\hline $\begin{array}{l}1.5 \text { Create leading-edge } \\
\text { scientific capabilities to } \\
\text { support evolving DOE } \\
\text { Mission needs }\end{array}$ & 3.93 & Outstanding & $10 \%$ & 0.39 \\
\hline $\begin{array}{l}\text { 1.6 Improve scientific impact } \\
\text { of EMSL user program }\end{array}$ & 3.85 & Outstanding & $10 \%$ & 0.39 \\
\hline & & \multicolumn{2}{|c|}{ Critical Outcome 1.0 Total } & 3.98 \\
\hline
\end{tabular}




\title{
Overall Self-Evaluation of Critical Outcome 2.0, Management and Operations Excellence

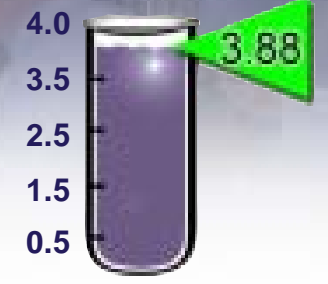

\begin{abstract}
Battelle managed and operated the Laboratory with distinction, becoming the DOE benchmark for Laboratory management, providing stewardship of DOE's assets, and protecting the health and safety of workers, the public, and the environment. In FY2003, PNNL met or exceeded nearly all DOE expectations in Management and Operations Excellence.
\end{abstract}

Battelle's evaluation of its performance under Critical Outcome 2.0, Management and Operations Excellence, indicates a score of 3.88 out of 4.0 , for a rating of Outstanding. Details of the evaluation are provided in Part I, Section 1 of this report. Adjectival and value ratings for Critical Outcome 2.0 and its supporting objectives and performance indicators are summarized in Appendix A, Tables A.8 - A.14.

\section{Highlights of the Three Performance Objectives}

2.1 Provide Management and Operational Excellence in Achieving Key Contract Performance Requirements

$\checkmark$ 2.1.1 Goal: Meet targets for 7 or more of 8 ESH\&Q measures. Result: PNNL met or exceeded targets for all 8 measures.

$\checkmark$ 2.1.2.1 Goal: Achieve overhead costs of $51 \%$ or less of the total 1830 average charge out rate. Result: PNNL achieved an overhead cost of 50.4\%.

$凶$ 2.1.2.2 Goal: Improve labor cost multiplier by $2 \%$ or more over FY2002. Result: PNNL improved labor cost multiplier by $1 \%$.

凶 2.1.2.3 Goal: Achieve FY2003 direct FTEs greater than or equal to $51 \%$ of total Laboratory FTE's. Result: PNNL achieved a direct FTE rate of $50.4 \%$.

$\checkmark$ 2.1.3 Goal: Achieve a composite score of $3.5-4.0$ for 11 ISSM indicators. Result: PNNL achieved a composite score of 3.6.

$\checkmark$ 2.1.4.1 Goal: Achieve a stewardship index of .98 or better. Result: PNNL achieved a stewardship index of 1.0.
2.1.4.2 Goal: Meet at least 6 of 7 energy conservation milestones. Result: PNNL met all 7 milestones.

2.2 Maintain and Enhance Laboratory Capabilities/Infrastructure to Meet Current and Future Mission Needs

$\checkmark$ 2.2.1.1 Goal: Meet all $6900 \mathrm{MHz}$ magnet milestones. Result: PNNL accomplished 6 of 6 actions.

$\checkmark$ 2.2.1.2 Goal: Bring Phase 1 and Phase 2 HP Supercomputer to full operational status. Result: PNNL met the goal.

$\checkmark$ 2.2.2.1 Goal: Establish an operating pilot proteomics facility in LSL II and characterize 4 or more complexes. Result: PNNL established the facility and characterized 5 complexes.

$凶$ 2.2.2.2 Goal: Complete all three deliverables critical to meeting computational sciences needs across major PNNL research areas. Result: PNNL completed (1) the requirements assessment and (2) procurement, but decided to postpone (3) hiring a Division Director.

$\checkmark$ 2.2.3.1 Goal: Complete all three Integrated Nuclear Strategy milestones and achieve Management Council decision on path forward. Result: PNNL met this goal.

2 2.3.2 Goal: Establish 4 or more joint nuclear R\&D projects with academia, or create 4 or more student positions. Result: PNNL met the goal by creating at least 22 new student positions; the stage is set for R\&D collaborations.

2.2.4.1 Goal: Meet at least 5 of 6 internet connection technology infrastructure milestones. Result: PNNL met 5 milestones. 
2.2.4.2 Goal: Meet at least 4 of 5 classified computing infrastructure milestones. Result: PNNL met 4 of 5 milestones.

2.2.4.3 Goal: Meet all 5 milestones relative to developing and implementing a Hanford 300 Area strategy. Result: PNNL met all 5 milestones.

\subsection{Provide Integrated Management Systems that Enable Effective and Efficient Business Performance}

2.3.1 Goal: Meet at least 8 of 9 milestones relative to selected improvement initiatives on the Laboratory's "Operations Improvement Agenda." Result: PNNL met all 9 milestones - and more.

Table 0.3. PNNL's overall self-evaluation of performance under Critical Outcome 2.0 indicates a score of 3.88 out of 4.0 , for a rating of "Outstanding."

\begin{tabular}{|l|c|c|c|c|}
\hline Element & $\begin{array}{c}\text { Total } \\
\text { Points }\end{array}$ & Adjectival Rating & $\begin{array}{c}\text { Objective } \\
\text { Weight }\end{array}$ & $\begin{array}{c}\text { Total } \\
\text { Points }\end{array}$ \\
\hline $\begin{array}{l}2.1 \text { Provide management and } \\
\text { operational excellence in } \\
\text { achieving key contract } \\
\text { performance requirements } \\
2.2 \text { Maintain and enhance } \\
\text { Laboratory capabilities/ } \\
\text { infrastructure to meet current } \\
\text { and future mission needs } \\
\begin{array}{l}2.3 \text { Provide integrated } \\
\text { management systems that } \\
\text { enable effective and efficient } \\
\text { business performance }\end{array}\end{array}$ & 3.81 & Outstanding & $50 \%$ & 1.91 \\
\hline
\end{tabular}




\section{Overall Self-Evaluation of Critical Outcome 3.0, Leadership Excellence}

PNNL has become recognized as a local, regional, and national asset. In FY2003, PNNL met or exceeded all but one of DOE's expectations in Leadership Excellence.

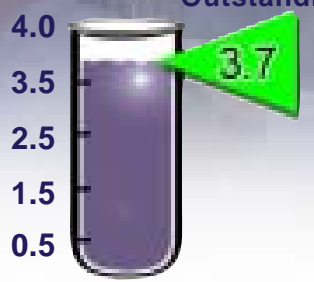

Result: PNNL assisted 46 firms and achieved a

Battelle's evaluation of its performance under Critical Outcome 3.0, Leadership Excellence, indicates a score of 3.70 out of 4.0, for a rating of Outstanding. Details of the evaluation are provided in Part I, Section 1 of this report. Adjectival and value ratings for Critical Outcome 3.0 and its supporting objectives and performance indicators are summarized in Appendix A, Tables A.15 - A.17.

\section{Highlights of the Three Performance Objectives}

\subsection{Attract, Develop, and Retain the Critical Staff Necessary to Achieve Simultaneous Excellence in S\&T, Operations, and Community Trust}

$\checkmark$ 3.1.1 Goal 1: Complete 50 StrengthsFinder assessments for Technical Group Managers (TGMs). Result: PNNL conducted 92 such assessments.

$\checkmark$ 3.1.1 Goal 2: Complete an analysis to define talent profiles for TGMs. Result: PNNL hired Gallup to conduct focus groups based on the first six of Gallup's Q12 characteristics of "Best Practices Managers."

$\checkmark$ 3.1.1 Goal 3: Define productivity measures for Research Division workgroups. Result: PNNL enlisted our Council of Fellows, which identified 12 productivity-defining characteristics for workgroups.

$凶$ 3.1.1 Goal 4: Increase the overall Laboratory workplace engagement assessment GrandMean score by .09. Result: PNNL achieved two-thirds of this stretch goal, increasing the score by .06.

\subsection{Demonstrate the Relevance of Pacific Northwest National Laboratory to the Needs of the Community and the Region}

$\checkmark$ 3.2.1.1 Goal: Start or expand a total of six or more businesses in the area where Battelle had a material role in their establishment. Result: PNNL achieved the goal of six.

$\checkmark$ 3.2.1.2 Goal: Provide technical assistance to at least 40 firms, with $85 \%$ of them reporting "very satisfied."

\author{
satisfaction rating of $100 \%$.
}

$\checkmark$ 3.2.1.3 Goal: Develop and champion at least one new economic development initiative to help grow and diversify the local and regional technology sector. Result: PNNL developed and implemented five such initiatives.

$\checkmark$ 3.2.2.1 Goal: Identify and describe how two technologies and/or capabilities from northwest institutions might address one or more regional needs. Result: PNNL focused on water resource management needs and identified 10 new capabilities.

$\checkmark$ 3.2.2.2 Goal: Develop a mechanism for commercializing an applicable technology or capability. Result: PNNL worked with students from the University of Oregon to develop a prize-winning business plan based on a water treatment technology.

$\checkmark$ 3.2.2.3 Goal: Formally present the Linking Regional Resources program and accomplishments to one governmental and one non-governmental agency in one or more states of the region. Result: PNNL made presentations to two governmental and two nongovernmental organizations.

$\checkmark$ 3.2.3 Goal: Generate at least $\$ 900,000$ in licensing revenue from DOE-derived inventions. Result: PNNL generated $\$ 1,326,746$ in such revenue, exceeding the mark by $47 \%$.

3.3 Impact Leadership and Diversity in Science and Engineering Education through Lab-Sponsored Programs for Students and Educators

$\checkmark$ 3.3.1 Goal: Receive an evaluation of 10 or higher out of 12 from $75 \%$ of LASER leadership teams surveyed about the impact of the program. Result: PNNL received the desired ratings from $93 \%$ of those surveyed.

$\checkmark$ 3.3.2 Goal: Achieve a 50\% increase in the diversity of the pool of applicants for PNNL project-funded fellowships. Result: PNNL far exceeded its outstanding target, achieving an increase of $76 \%$. 
Table 0.4. PNNL's overall self-evaluation of performance under Critical Outcome 3.0 indicates a score of 3.70 out of 4.0 , for a rating of "Outstanding."

\begin{tabular}{|c|c|c|c|c|}
\hline Element & $\begin{array}{l}\text { Total } \\
\text { Points }\end{array}$ & Adjectival Rating & $\begin{array}{c}\text { Objective } \\
\text { Weight }\end{array}$ & $\begin{array}{l}\text { Total } \\
\text { Points }\end{array}$ \\
\hline $\begin{array}{l}3.1 \text { Attract, develop and retain } \\
\text { the critical staff necessary to } \\
\text { achieve simultaneous } \\
\text { excellence in } S \& T \text {, operations, } \\
\text { and community trust }\end{array}$ & 3.00 & Excellent & $30 \%$ & 0.90 \\
\hline $\begin{array}{l}\text { 3.2 Demonstrate the relevance } \\
\text { of Pacific Northwest National } \\
\text { Laboratory to the needs of the } \\
\text { community and the region }\end{array}$ & 4.00 & Outstanding & $50 \%$ & 2.00 \\
\hline $\begin{array}{l}3.3 \text { Impact leadership and } \\
\text { diversity in science and } \\
\text { engineering education through } \\
\text { Lab-sponsored programs for } \\
\text { students and educators }\end{array}$ & 4.00 & Outstanding & $20 \%$ & 0.80 \\
\hline & & \multicolumn{2}{|c|}{ Critical Outcome 3.0 Total } & 3.70 \\
\hline
\end{tabular}





\section{Contents}

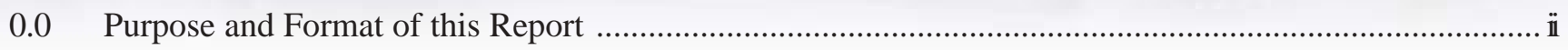

\section{Executive Summary}

$0.1 \quad$ Basis Battelle's “Outstanding” Rating in FY2003 _................................................................................. vi

0.2 Overall Self-Evaluation of Critical Outcome 1.0, Scientific and Technological Excellence .................... viii

0.3 Overall Self-Evaluation of Critical Outcome 2.0, Management and Operations Excellence .....................

0.4 Overall Self-Evaluation of Critical Outcome 3.0, Leadership Excellence ............................................... xii

\section{Part I-Status of Performance Against Critical Outcomes, 1.0 Science and Technology Excellence}

1.0 Status of Performance Against Critical Outcomes, 1.0 Science and Technology Excellence PNNL's FY2003 Performance on Behalf of DOE Missions ..........................................................2

1.0.1 FY2003 Recognition for PNNLAccomplishments: Publications, and Professional Honors .....................4

1.0.2 FY2003 Recognition for PNNL Accomplishments: Technology Awards ............................................... 6

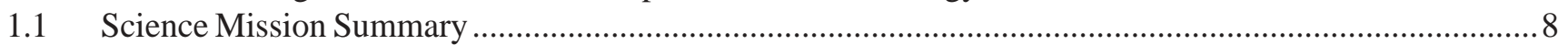

1.1.1 Customer and Peer Review of PNNL Asset Enhancements to Better Accomplish the SC Mission ....... 10

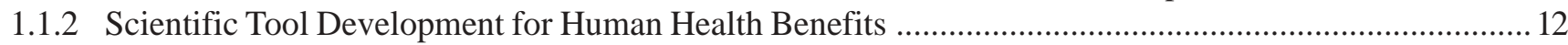

1.1.3 Potential Benefits of Single-Chain Antibody Technology Development ............................................. 14

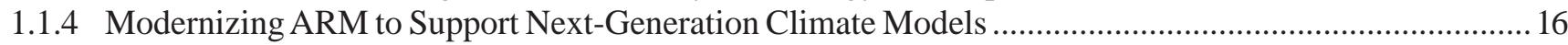

1.1.5 Advanced Simulation Software for Subsurface Science ….............................................................. 18

1.1.6 (PEFA 1.3 and 1.6.1) Science Grand Challenges to Increase Impact of EMSL User Program .............20

1.1.7 (PEFA 1.3 and 1.6.2) Optimal Model for EMSL User Facility Operations (User Program) ....................22

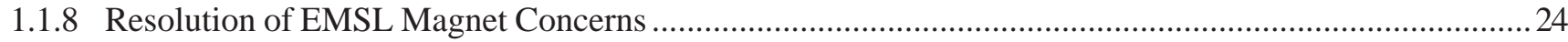

1.1.9 Benefits of New EMSL User Project and Resource Management Systems ......................................26

1.1.10 (PEFA 1.5.1) Continuing to Build a Strong Multidisciplinary Program in Systems Biology ......................28

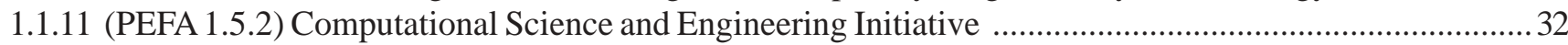

1.1.12 (PEFA 1.5.3) Nanoscience and Technology Initiative: Applications-Driven Fundamental Science ..........34

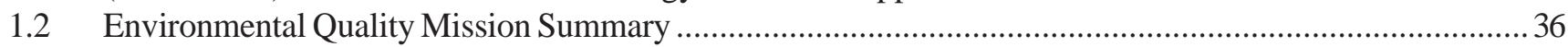

1.2.1 Results of Environmental Technology Directorates Division Review Committee Review .......................38

1.2.2 PNNL's Research and Development and Assessment Projects and their Relevance to Hanford's Groundwater Protection Mission .......................................................40

1.2.3 Hanford Site Work on Tank Waste, Safe Storage and Retrieval,

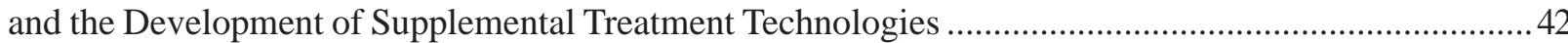

1.2.4 PNNL's Work in Support of Hanford's Central Plateau Restoration Activities ......................................44

1.2.5 PNNL's Program Management Performance in Support of RL and the Office of River Protection ....... 46

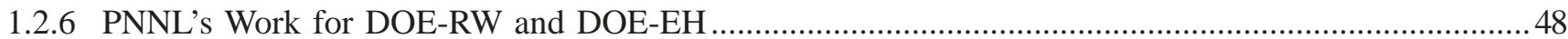

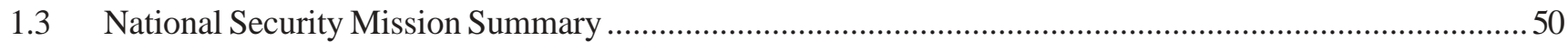

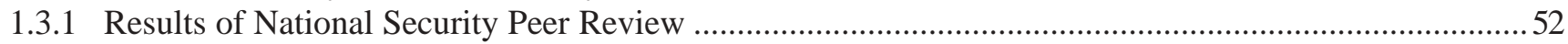

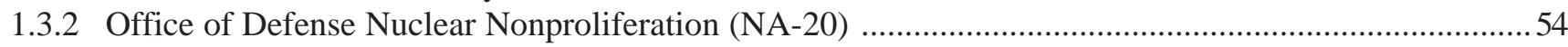

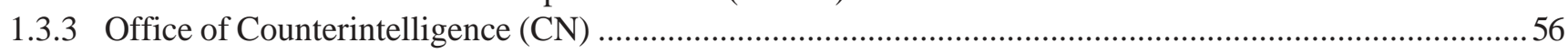

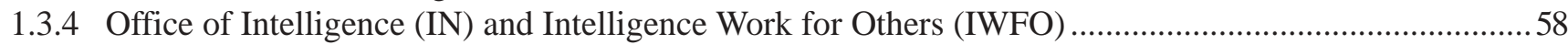

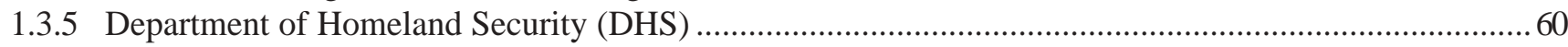

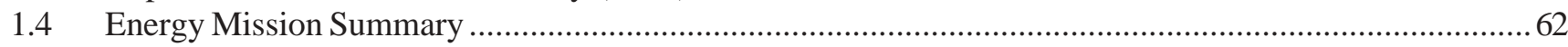

1.4.1 Validating Laboratory Energy Science and Technology through External Peer Review ........................64

1.4.2 Advancing Developments in Distributed Energy and Energy Systems ...............................................66

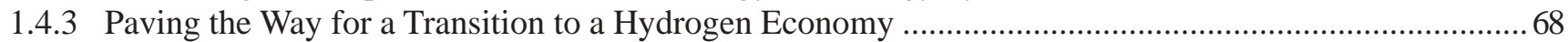

1.4.4 Advancing Transportation Technologies for High-Efficiency Transportation Systems ...........................70

1.4.5 Creating Innovative and Economically Viable Bio-Based Products and Processes ................................ 72 
1.4.6 Developing Technologies for Clean and Efficient Power Generation

1.4.7 Support of DOE Energy and National Security Missions with Advanced Nuclear Science and Technology

Part I-Status of Performance Against Critical Outcomes, 2.0 Management and Operations Excellence

2.0 Status of Performance Against Critical Outcome 2.0: Management and Operations Excellence ...........8 80

2.1.1 ES\&H Management Systems that Sustain and Enhance Excellence in Laboratory Operations ...............82

2.1.2 Performance Against Financial Management Metrics ...................................................................... 84

2.1.3 Sustaining the Effectiveness of Integrated Safeguards and Security ................................................ 86

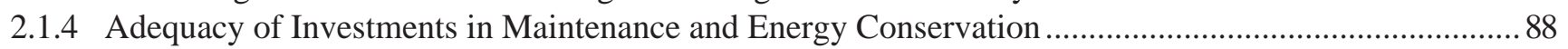

2.2.1 Enhancing EMSL to Support the User Community ........................................................................... 90

2.2.2 Status of Systems Biology and Computational Capabilities Required to Realize Strategy .......................99

2.2.3 Aligning PNNL's Nuclear Capability with Future Needs .....................................................................94

2.2.4 Identifying and Providing IT and Physical Infrastructure to Support PNNL's Strategy .........................96

2.3.1a Commitments to Improve the Effectiveness and Efficiency

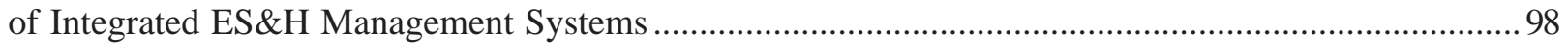

2.3.1b Commitments to Improve the Effectiveness and Efficiency of Business-Related Management Systems

Part I-Status of Performance Against Critical Outcomes, 3.0 Leadership Excellence

3.0 Status of Performance Against Critical Outcome 3.0: Leadership Excellence .................................... 104

3.1.1 How PNNL Creates a Great Place to Work ............................................................................... 106

3.2.1 PNNL's Role in Growing and Diversifying the Local and Regional Economy ................................... 108

3.2.2 PNNL's Role in Linking Regional Resources ............................................................................... 110

3.2.3 Enhancing the Laboratory's Ability to Generate Revenues from Commercialization ............................ 112

3.3.1/3.3.2 PNNL Programs for Leadership and Diversity in Science and Engineering Education ................. 114

Part II-Strengths and Opportunities for Improvement

II.1 Strengths and Opportunities for Improvement .............................................................................. 118

Part III-Adequacy of PNNL's Integrated Assessment Processes

III.1 Adequacy of Integrated Assessment Performance to Establish Compliance

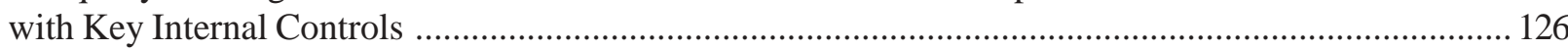

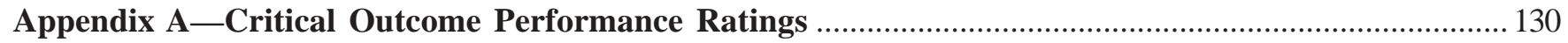

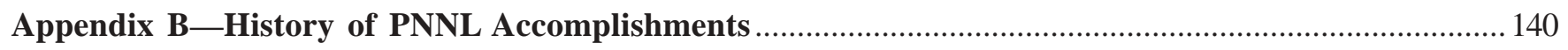

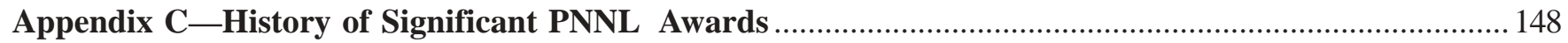

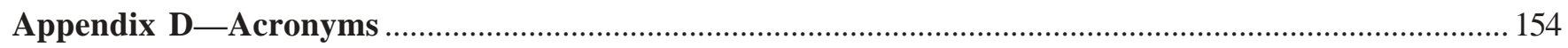




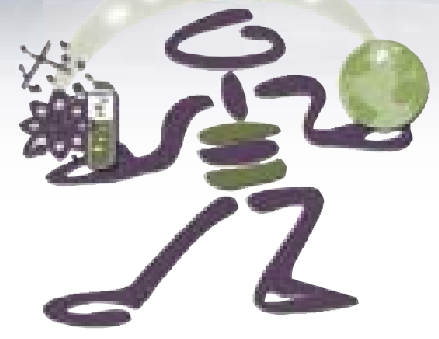

\section{Part I \\ Status of Performance Against Critical Outcomes 1.0 Science and Technology Excellence}

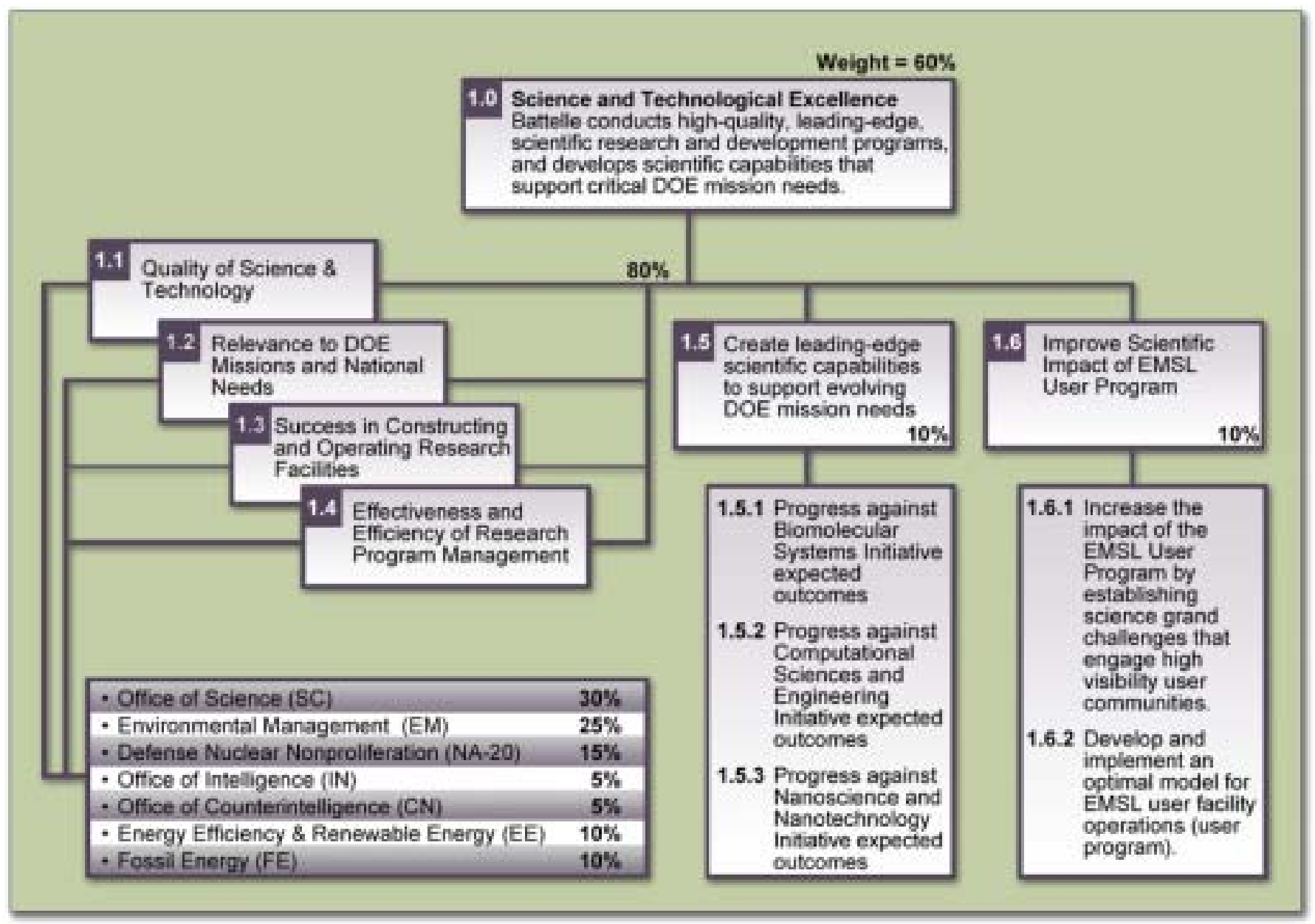




\subsection{Status of Performance Against Critical Outcome 1.0: Science and Technology Excellence-PNNL's FY2003 Performance on Behalf of DOE Missions}

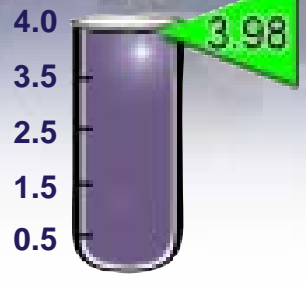

\section{PNNL conducts high-quality, leading-edge scientific and technological research and development programs that are recognized by the scientific community, and creates scientific capabilities and user programs that support critical DOE mission needs and address other national priorities.}

\section{FY2003 Recognition for the Relevance and Quality of PNNL Accomplishments}

The Laboratory received significant external recognition in FY2003, including three R\&D 100 and three FLC awards. PNNL ranks fifth and first, respectively, among the other multiprogram labs in R\&D 100 and FLC awards. The quality of our scientific efforts is reflected by the list of staff that were recognized for their scientific and engineering excellence in terms of awards, invited talks, and participation on scientific committees. In addition, our publication rate is the highest ever, and eight of our top 10 publication venues are among the top ten journals in their respective fields.

\section{Science Mission - (Section 1.1)}

The Fundamental Science Directorate (FSD) manages PNNL's role in DOE's Science mission and is the primary customer interface for DOE's Office of Science.

Researchers in FSD are

- Making advances in mass spectrometry technology to enable progress across a broad spectrum of biological research, including diagnoses of breast cancer, prostate cancer, and heart disease;

- Making advances in single-chain antibody technology, which could have major impacts on fundamental biological science as well as industries that use antibodies for sensors, biodetectors, diagnostic tools, and therapeutic agents;

- Using parallel processing to address large, highresolution, three-dimensional subsurface multiphase problems;
- Increasing the impact of EMSL as a user facility;

- Building a strong foundation to apply systems biology methods and transition to systems biology workflows;

- Increasing the visibility of PNNL's computational science; and

- Creating the capability to manipulate structures at an atomic scale to fundamentally change the properties of materials and make possible new materials, chemistry, and functions.

\section{Environmental Quality Mission - (Section 1.2)}

The Environmental Technology Directorate (ETD) manages PNNL's role in DOE's Environmental Quality mission and is the primary customer interface for DOE's Assistant Secretary for Environmental Management (EM).

Researchers in ETD are providing science and technology to

- Make sound decisions necessary to protect and remediate Hanford Site groundwater and the Columbia River;

- Ensure the safe storage and retrieval of Hanford's radioactive tank wastes;

- Resolve critical spent nuclear fuel issues and enable the stabilization and safe storage of Hanford's plutonium;

- Provide radiation protection services for DOE-RL and Hanford contractors; and

- Support the nation's High-Level Waste Repository and protect the workers, the public, and the environment. 


\section{National Security Mission - (Section 1.3)}

The National Security Directorate (NSD) manages PNNL's role in DOE's National Security mission and is the primary customer interface for the Department of Homeland Security (DHS) and three key DOE-HQ Program Offices: the Office of Defense Nuclear Nonproliferation (NA-20); the Office of Intelligence (IN); and the Office of Counterintelligence $(\mathrm{CN})$.

\section{Researchers in NSD are}

- Addressing U.S. national security challenges of detecting and preventing proliferation of weapons of mass destruction;

- Conducting counterintelligence activities, developing technologies, and deploying cyber security tools for DOE;

- Providing analytical support for DOE-IN and other armed services and national intelligence agencies; and

- Addressing U.S. challenges of sensing, analysis, and evaluation within radiological, biological, and chemical national requirements with the objective of detecting the unexpected.

\section{Energy Mission - (Section 1.4)}

The Energy Science \& Technology Directorate (ESTD) manages PNNL's role in DOE's Energy Resources mission and is the primary customer interface for the Assistant Secretary for Energy Efficiency and Renewable Energy (EERE), Assistant Secretary for Fossil Energy (FE), and Office of Nuclear Energy (NE).

Researchers in ESTD continue to demonstrate leadership, technical quality, innovation, and impact in converting, transporting, and using energy consistent with national priorities and DOE's Energy missions and program plans. They are

- Leading in the development of advanced communication and control technologies to benefit a highly connected electricity grid and distributed energy systems;

- Developing science and technology solutions that will bridge the gap to a hydrogen economy;

- Developing advanced transportation technologies for essential power systems, lightweight materials, and emissions aftertreatment;

- Developing bio-based products and processes to displace imported petroleum and implement integrated biorefineries;

- Advancing the objectives of the DOE FutureGen Initative; and

- Sustaining and building national capabilities in nuclear science and engineering.

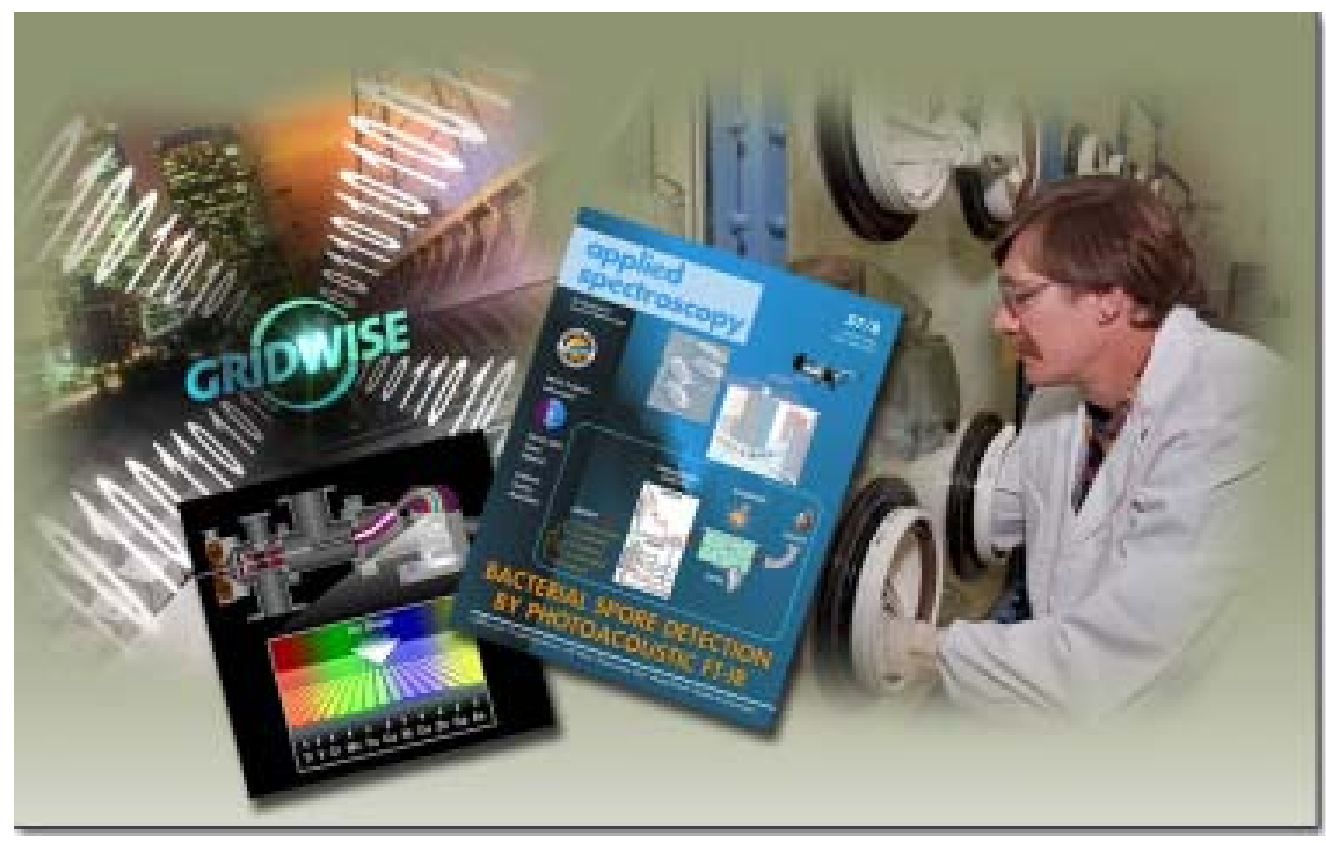

Figure 1.0. PNNL's contributions to fundamental science, environmental quality, national security, and energy continue to place the Laboratory at the forefront of human knowledge and capability. 


\subsubsection{FY2003 Recognition for PNNL Accomplishments: Publications, and Professional Honors}

\section{Prestigious awards, publications in important journals, and recognition by professional peers attest to PNNL's continuing success in producing original, creative, and relevant science and technology.}

\section{Publications in Peer- Reviewed Journals}

PNNL researchers produced a record 715 publications, making FY2003 our most prolific year ever. Of these publications, which include 567 articles in peer-reviewed journals, 11 book chapters, and 137 conference proceedings, we emphasize articles accepted by journals with a high impact factors, based on citation rates, as determined by Thompson ISI ${ }^{\circ}$. As shown in Figure 1.0.1 eight of the top ten publication venues that PNNL authors selected have impact factors that place these journals among the top ten journals in their respective fields.

\section{Recognition by Professional Peers}

\section{Professional Societies/Honors}

John Abrefah was appointed a member of the Meetings, Proceedings, and Transactions Committee and Honors and Awards Committee of the American Nuclear Society.

Obie Amacker was awarded the Meritorious Achievement Award of the Institute of Nuclear Materials Management.

Michael Bowman received the Silver Medal in Chemistry by the International EPR/ESR Society.

Greg Exarhos has been named a Fellow in the American Vacuum Society

Darrell Fisher and Paul Stansbury were elected Fellows of the Health Physics Society.

Linda Lasure received the Charles Porter Award from the Society of Industrial Microbiology for her exceptional record of sustained services to the society.

Walt Laity was elected Vice President, Engineering Education of the American Society of Mechanical Engineers.
Pete Martin was elected a Mentor/Fellow of the Society of Vacuum Coaters.

Dean Matson was awarded the Regional Industrial Innovation Award by the American Chemical Society.

Prabhakar Singh was elected a Fellow in the American Ceramic Society.

Subhash Singhal was appointed President (2004-2006) of the International Society for Solid State Ionics.

Sriram Somasundaram was appointed to the Advanced Energy Systems Division Chair of the American Society of Mechanical Engineers.

Darby Stapp has been named a Fellow in the Society for Applied Anthropology.

John Zachara was recognized as a highly cited researcher in ecology/environment by the Institute for Scientific Information.

\section{Governmental Awards}

J. David Briggs received the Information Classification and Control Policy Award from the DOE Office of Security.

\section{Other Honors/Appointments}

Cindy Bruckner-Lea was selected as a participant at the National Academy of Engineering Frontiers of Engineering Symposium.

Jean Futrell was appointed to the 1) Chemical Sciences Roundtable of the National Research Council of the National Academy of Sciences; 2) Board on Chemical Sciences and Technology of the National Research Council of the National Academy of Sciences; and 3) Scientific Advisory Board for High Magnetic Fields Laboratory of the National Science Foundation.

Sotiris Xantheas received the Friedrich Wilhelm Bessel Research Award from the Alexander von Humboldt Foundation. 


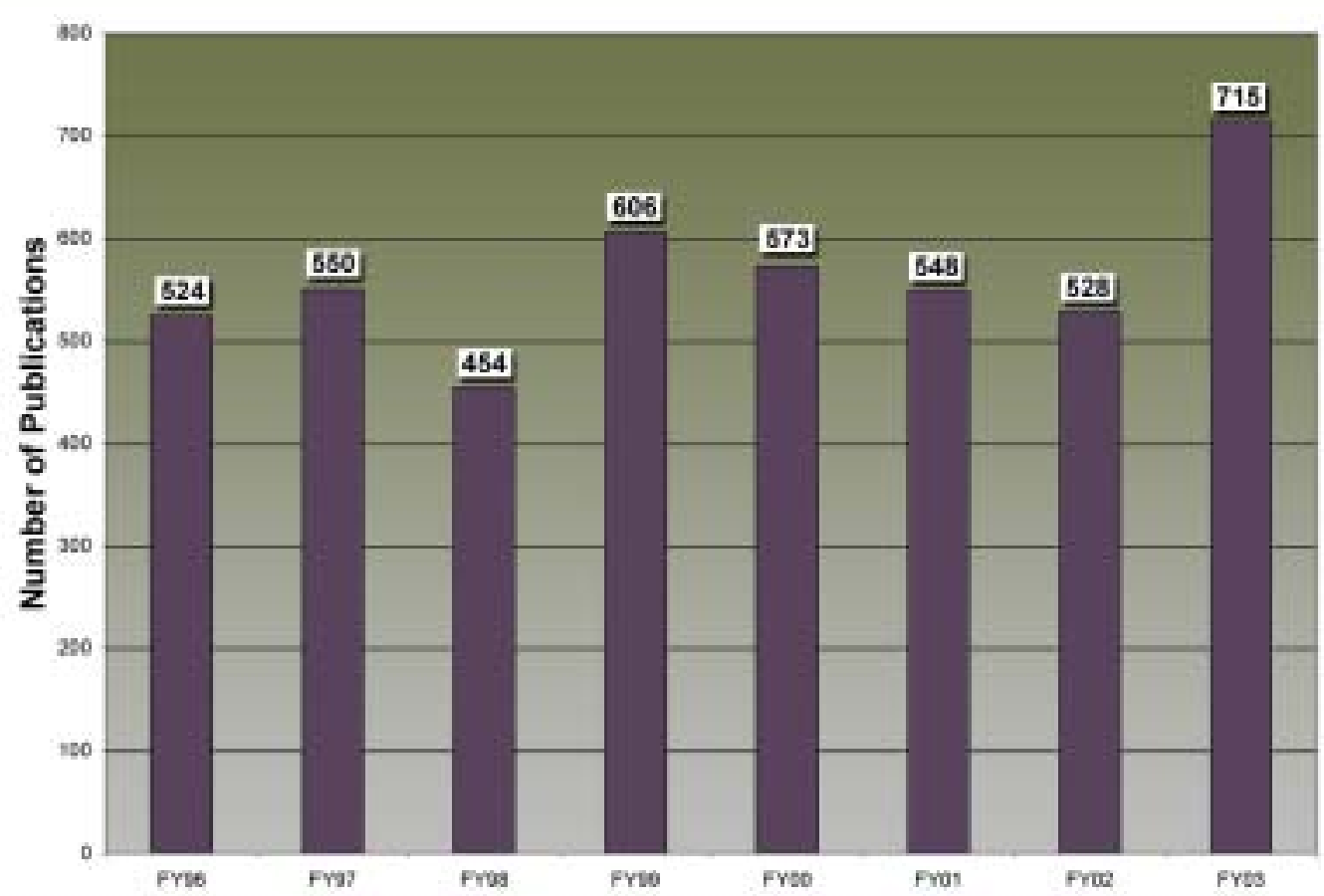

Selected Journal Titles

PNNL Articles FY2003

*Journal of Nuclear Materials

*Journal of Chemical Physics

Journal of Physical Chemistry A

Journal of Geophysical Research-Atmospheres (Section D)

${ }^{*}$ Geochemica et Cosmochimica Acta

*Environmental Science and Technology

*Journal of Physical Chemistry B

*Journal of the American Chemical Society

*Analytical Chemistry

*Nuclear Instruments and Methods in Physics Research B

Figure 1.0.1. Technical results are largely communicated through peer-reviewed publications. Publications include journal articles, conference papers, and book chapters. In FY2003, the Lab published 715 articles compared to 528 in FY2002. (Astericks indicate Thompson ISI ${ }^{\circledR}$ "top ten" status.) 


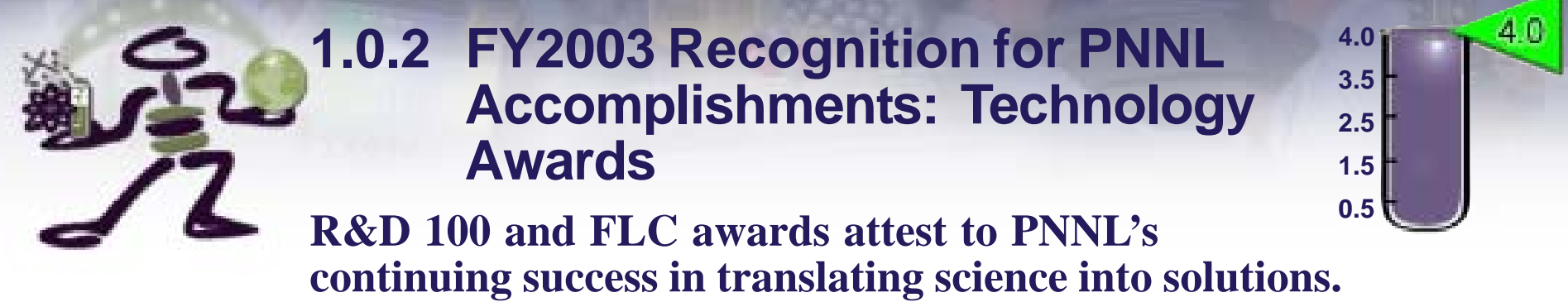

\section{Technology Awards}

\section{R\&D 100 Awards}

Three of the five entries PNNL submitted this year received awards. PNNL ranks fifth in all federal laboratories in the number of R\&D 100 Awards won (62). Brief descriptions of the three awards follow:

FT-MS Proteome Express-This breakthrough technology both significantly accelerates proteome analysis and also provides unprecedented accuracy and depth. It is the first-ever high-throughput Fourier-transform ion cyclotron resonance mass spectrometer (FT-MS), with a unique ability to characterize and identify proteins, especially small quantities. Providing quantitative analyses of "proteomes," collections of proteins that make up cells or organisms under specific conditions at a specific time, it can be applied to understanding the role of proteins in diseases, such as cancer, and provides a basis for developing treatment drugs. This technology will revolutionize our ability to understand biological systems and to develop biotechnological solutions for the nation's most pressing energy and environmental problems.

Product Acoustic Signature System (PASS)_PASS is an acoustic inspection device using ultrasonic pulse echo technology to non-intrusively identify the contents of sealed containers. Using PASS, Customs inspectors can tell if the tanker truck or barrel in front of them contains crude oil, vegetable oil, or chemical weapons agents. PASS can also detect hidden packages and compartments and determine the container's fill level, avoiding long, potentially hazardous physical sampling and searches. Developed at PNNL, PASS is licensed to Mehl, Griffin, \& Bartek, Ltd., for manufacturing and marketing. A valuable tool for identifying weapons of mass destruction, preventing smuggling, enforcing tariffs, and deterring illicit drug trafficking, PASS provides one of the safest, simplest means available to keep commerce moving safely, support homeland security, and perform verification activities to help maintain world stability.
Starlight Information Visualization System-Starlight launches a new generation in visualization technology by uncovering key relationships hidden in large, complex, dynamic information collections. A unique analysis tool, Starlight integrates structured, unstructured, spatial, and multimedia data, comparing information at multiple levels of abstraction simultaneously and in near real-time, capturing and graphically depicting complex relationships in data from multiple sources. By making such relationships simultaneously visible, Starlight enables exciting, rapid, and powerful new forms of concurrent information exploitation. The result is an unprecedented approach to information management and sensemaking.

\section{FLC Awards}

This year, all three entries received awards. PNNL continues to lead federal laboratories in the number of Federal Laboratory Consortium (FLC) Awards won (54). Brief descriptions of the awards follow:

Acoustic Inspection Device-This is another way of referring to the Product Acoustic Signature System (PASS), which won an R\&D 100 Award and is thus doubly distinguished.

EMADVANTAGE: Emergency Management Capabilities to Support Multiple Users and Jurisdictions-In an emergency, getting the right information to the right people in time to make the right decisions can save lives and property. PNNL developed an emergency management software system that accomplishes that goal and transferred it to a refinery in Mexico, a small business in Maryland, the National Aeronautics and Space Administration (NASA), and the U.S. Army. The system is based on components of the Federal Emergency Management Information System (FEMIS), the breakthrough technology PNNL developed to safeguard communities near chemical weapons depots. Recognizing the need for a general operations and emergency management system for natural disasters and human-caused 
hazards, the PNNL nominees enhanced, copyrighted, and packaged FEMIS into a suite of capabilities called EMADVANTAGE, the only system that supports all phases of emergency management and makes information available with constant, dynamic updates via desktop computer, the Internet, and personal digital assistants (PDAs).

\section{Engine Exhaust After-Treatment System Based on Non-Thermal Plasma-Assisted Catalysis-PNNL} and its industry partners have developed an engine exhaust after-treatment system that converts harmful oxides of nitrogen (NOx) and particulate matter (PM) into components of clean air. PNNL's system, based on non-thermal plasma- (NTP) assisted catalysis, addresses industry's serious need for a technology that will meet 2010 regulations for dramatic reductions in these emissions. NOxs react with water vapor in the atmosphere to form acid rain and are a precursor to ozone, a major component of smog. PMs cause respiratory irritation and can contribute to chronic health effects. The need for a technology to reduce these emissions is so great that the nominees successfully transferred the technology to three organizations: Delphi Corp., Caterpillar Inc., and the Low Emissions Partnership of USCAR, a government-industry program that includes Ford, DaimlerChrysler, and General Motors.

Table 1.0.2. PNNL ranks fifth and first, respectively, among the other multiprogram labs in R\&D 100 and FLC Awards.

\begin{tabular}{|l|c|c|}
\hline \multicolumn{2}{|c|}{ R\&D 100 and Federal Laboratory Consortium Awards by National Laboratory } \\
\hline Multiprogram Laboratory & $\begin{array}{c}\text { R\&D Awards } \\
(1964-2003) \text { (rank) }\end{array}$ & $\begin{array}{c}\text { FLC Awards } \\
(\mathbf{1 9 8 4 - 2 0 0 3 )} \text { (rank) }\end{array}$ \\
\hline Argonne & $67(4)$ & $22(4)$ \\
Brookhaven & $23(10)$ & $11(9)$ \\
Idaho & $30(9)$ & $8(11)$ \\
Lawrence Berkeley & $35(7)$ & $20(5)$ \\
Lawrence Livermore & $92(2)$ & $27(3)$ \\
Los Alamos & $88(3)$ & $13(7)$ \\
Oak Ridge & $116(1)$ & $33(2)$ \\
Pacific Northwest & $62(5)$ & $\mathbf{5 4}(1)$ \\
Sandia & $61(6)$ & $14(7)$ \\
\hline
\end{tabular}

Note: For a comprehensive history of PNNL accomplishments and awards, please see Appendix B and C. 


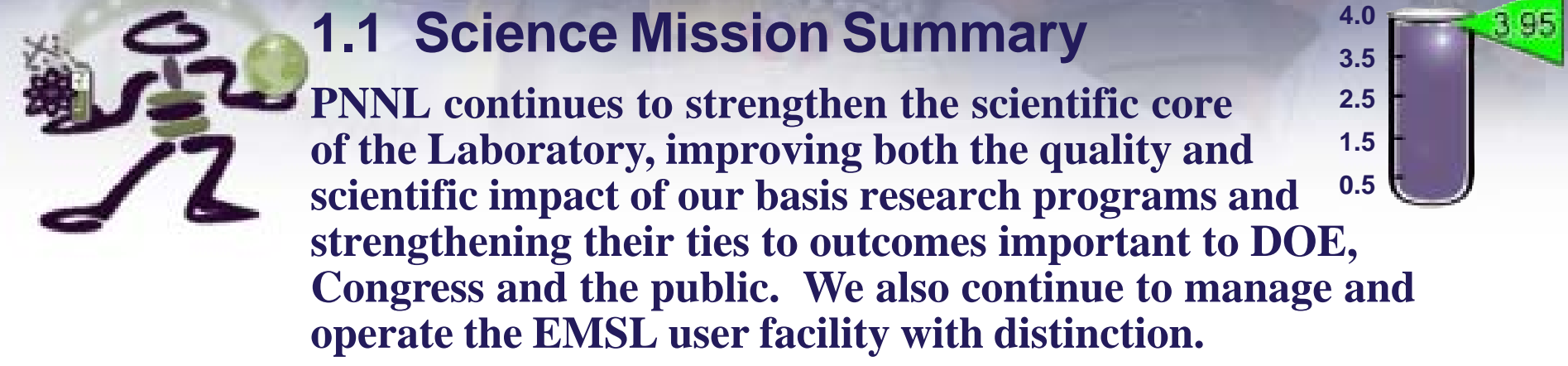

The modules in this section present examples of quality, relevance, user facility operation, and research program management from the perspective of PNNL's science mission. Modules under this mission are summarized below into the categories of objectives 1.1 - 1.6: Quality, Relevance, Research Facilities, Research Program Management, Leading-Edge Scientific Capabilities, and EMSL User Program.

\section{PEFA Objectives 1.1 and 1.2 - Summary of Modules under Quality and Relevance}

1.1.1 Peer Review. Our major SC customers and the Directorate Review Committee provided us with beneficial feedback on the year's efforts. In FY2003, PNNL made sweeping strategic and tactical moves to better accomplish DOE's Office of Science mission, including reorganizing FSD to better align with our customers and revamping the user interface in EMSL to more actively seek out users and projects. EMSL maintained productivity during these transitions as demonstrated by the continuation of user and staff publications, awards and honors described in monthly reports on the external EMSL website (http://www.emsl.pnl.gov/ docs/monthly_reports.shtml). A paper by Thompson SE, NS Foster, TJ Johnson, NB Valentine, and JE Amonette entitled "Identification of Bacterial Spores Using Statistical Analysis of Fourier Transform Infrared Photoacoustic Spectroscopy Data," featured on the cover of Applied Spectroscopy 57(8):893-899 (Figure 1.1) is an excellent example.

1.1.2 Scientific Tool Development for Human Health BENEFITS. Advances in mass spectrometry technology at PNNL are enabling progress across a broad spectrum of biological research, including diagnoses of breast cancer, prostate cancer, and heart disease. With DOE-BER support, PNNL established a high-throughput proteome analysis pilot production line that is expected to serve as a precursor for additional lines planned for FY2004. This development effort sets the stage for PNNL to compete for the GTL whole proteome analysis facility.

1.1.3 Potential Benefits of Single-Chain Antibody Technology Development. Continued advances in single-chain antibody technology during FY2003 hold promise for precise, rapid (days, not months) detection across a range of applications from cancer to national security.

1.1.4 Modernizing ARM to Support NeXt-Generation Climate Models. Three major accomplishments highlight PNNL's FY2003 ARM performance: the ARM data system modernization has been completed; remote sensing techniques have been developed to determine properties of mixed-phase cloud properties; and new "superparametrization" techniques greatly increase the physical realism of the representation of clouds in climate models.

1.1.5 Advanced Simulation Software for Subsurface SCIENCE. With this LDRD project, PNNL significantly improved its ability to use parallel processing to address large, high-resolution, three-dimensional subsurface multiphase problems.

\section{PEFA Objectives 1.3 and 1.6 - Summary of Modules under Research Facilities}

1.1.6 (PEFA 1.6.1) Science Grand Challenges to Increase Impact of EMSL User Program. Two science grand challenges, one in biogeochemistry (subsurface science) and one in biology, are on schedule to be established by early FY2004 and will greatly increase the impact of the EMSL user program. The scientific grand challenges are designed to leverage integrated sets of EMSL research capabilities in the study of highly significant scientific problems. The grand challenges, 
which will last three to five years, will create new benchmarks for integrated research. These challenges will be aligned with DOE mission areas, will be driven by users, and will take full advantage of EMSL's unique capabilities, resources, and technical expertise. These scientific challenges are designed to attract and involve users who are among the best scientists in the world in the area of the challenge and will greatly increase the impact of the EMSL user program.

1.1.7 (PEFA 1.6.2) Optimal Model For EMSL User Facility Operations (User Program) A new EMSL user operations model is already increasing the scientific impact of this unique resource. In late FY2002, EMSL staff visited several user facilities to identify best practices in preparation for revamping the operation of EMSL. After evaluating the selected facilities, PNNL, in close coordination with OBER, the EMSL User Advisory Committee, and PNSO, developed a model combining the best features of each. Submitted to OBER and BERAC in FY2003, the model became the basis for a detailed EMSL Facility Operations Manual.

\section{PEFA Objective 1.4 - Summary of Modules under Research Program Management}

1.1.8 Resolution of EMSL Magnet Concerns. FSD effectively managed concerns about safe operation of high field magnets in EMSL through immediate action and outside expert review of operations.

1.1.9 Benefits of New EMSL User Project and Resource Management Systems. EMSL has greatly improved both the effectiveness and efficiency of its management of user projects and resources by implementing two new software applications.

\section{PEFA Objective 1.5 - Laboratory Initiatives}

\subsubsection{0 (PEFA 1.5.1) Continuing to Build a Strong} Multidisciplinary Program in Systems Biology. In the course of meeting or exceeding all the BSI targets, PNNL was especially fortunate to recruit George Michaels, an exceptional leader in bioinformatics science around whom we are building a solid foundation to apply systems biology methods and transition to systems biology workflows.

1.1.11 (PEFA 1.5.2) Computational Science And EngINEERING InITIATIVE (CS\&EI) ACCOMPLISHMENTS. The CS\&EI achieved all six technical research milestones, building capability across a broad spectrum of research areas and making significant strides in increasing the visibility of PNNL's computational science.

1.1.12 (PEFA 1.5.3) Nanoscience and Technology Initiative: Applications-Driven Fundamental Science. The Nanoscience and Technology Initiative continues to gain visibility for creating the capability to manipulate structures at an atomic scale to fundamentally change the properties of materials and make possible new materials, chemistry, and functions.

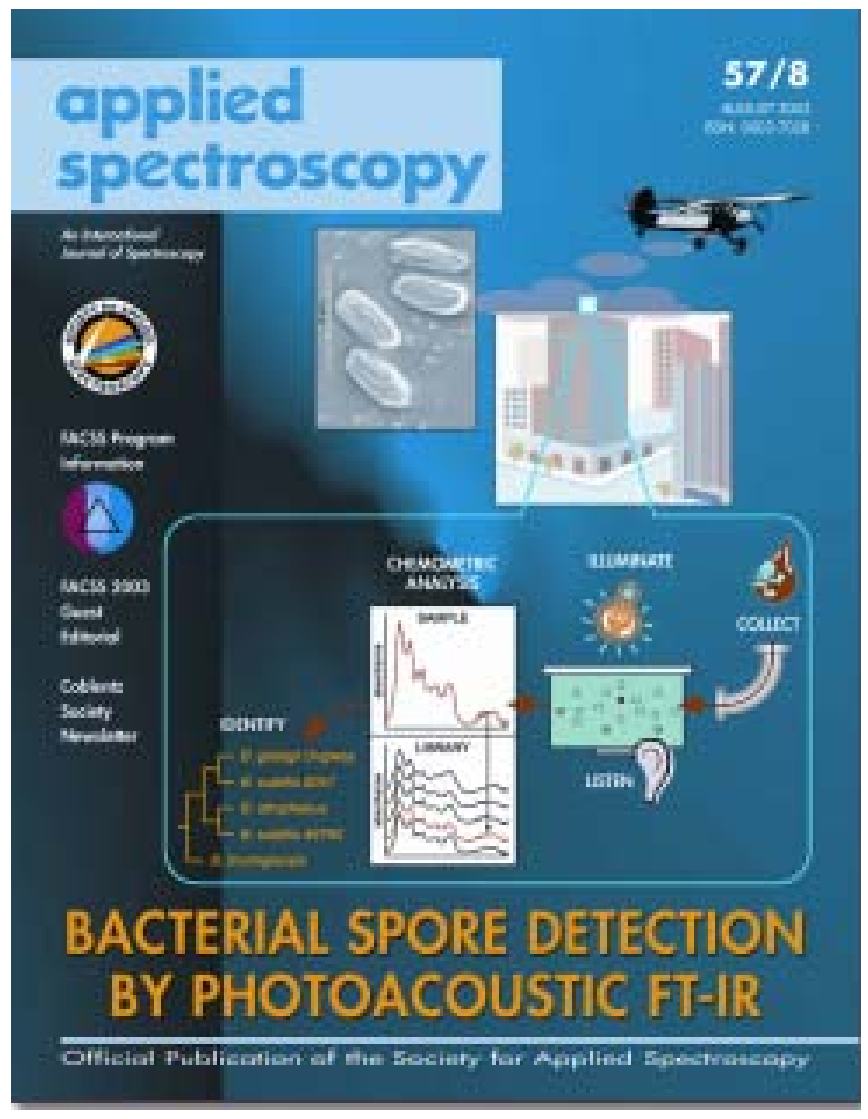

Figure 1.1. New technique for identifying strains of bacterial spores requires a minimum of test sample preparation and provides highly accurate results. The instrumentation has the potential to be made portable for testing samples in the field. 


\subsubsection{Customer and Peer Review of PNNL Asset Enhancements to Better Accomplish the SC Mission}

\section{In FY2003 PNNL made sweeping strategic and tactical moves to better accomplish DOE's Office of Science mission. Our major SC customers and the Directorate Review Committee (DRC) provided beneficial feedback on the year's efforts.}

\section{Context for Science Mission Peer Review}

In FY2003, FSD reorganized to better align with its major customers, and EMSL revamped its user interface to more actively seek out users and projects that would reinforce its research vision and enhance its reputation. The new user philosophy is an active outreach to highprofile scientists in areas that will lead to optimum use of the facility and staff. This effort includes:

- A sabbatical program to entice senior scientists. (see module 1.1.7)

- Establishment of EMSL grand challenges (see module 1.1.6)

- New web-based project proposal and tracking system tool to improve project selection and user accountability (see module 1.1.9)

- New database system to better track and schedule equipment usage (see module 1.1.9)

Concurrently, the Lab pushed hard to attract new research complementary to its capabilities and mission. Examples include the NCRR Proteomics Research Resource, a significant NIH win that should lead to future NIH research; an important multi-institutional project win in catalysis science entitled Early transition metal oxides as catalysts: Crossing scales from clusters to single crystals to functioning materials; and the well-received proposal to BES for a possible future Complex Interfacial Catalysis Facility.

Meanwhile, the Atmospheric Sciences and Global Change Division redirected its focus to embrace the shift in DOE emphasis from atmospheric chemistry to aerosols, positioning continued for PNNL's part in GTL, the $900 \mathrm{MHz}$ NMR was brought to field, and the HPCS2 supercomputer was brought on line at its 11.8 Teraflop capacity. These are examples of the changes taking place as PNNL addresses DOE's challenges and the nation's fundamental science needs. The acceleration of major strategic and tactical moves over the past few years is striking. The timeline in Figure 1.1.1 identifies only a sampling of these achievements, but provides perspective on the rapidly increasing pace of change.

\section{Phone Interviews with BER, BES and ASCR}

Given all this activity, it behooves FSD to ask our major SC customers and external peer reviewers (DRC) how we are doing. We spoke with BER Associate Director Ari Patrinos. BER, very pleased with the quality of FSD leadership at all levels, is particularly impressed with improvements to communications between SC and PNNL, which make problem solution possible.

PNNL must collaborate effectively to mitigate the liability of its geographical isolation. A lot of progress has been made in PNNL's collaboration with universities, and BER has high hopes for more given new Lab Director Len Peters' academic background and intention for enhanced university partnerships.

BER is impressed and satisfied with the responsiveness and support of PNNL and Battelle leadership. It is also pleased with the partnership between BER and PNSO, indicating that it is a "real pleasure" to work with the site office.

From BES we spoke to Chemical Sciences, Geosciences, and Biosciences Division Director Walt Stevens, who believes that PNNL has outstanding scientific leaders, especially in Chemical Physics. Overall staff quality rates high. Walt considers PNNL well above the average of the other Labs and exceptional in some areas. 
BES considers PNNL to be quite good at teaming with universities and other Labs. Implementing our scientific stretch goals should make things even better. The high rating given to the catalysis proposal shows the esteem in which BES holds PNNL.

Laboratory institutional support for BES programs is considered excellent. Early concerns that BES might suffer as a result of the FSD reorganization have been dispelled largely through FSD Chemical Sciences Division Director Doug Ray's efforts.

BES is extremely pleased with how PNNL manages BES programs. Walt commended Doug Ray in particular, as our Chemical and Material Sciences Director responsible for our primary interface with BES, and noted that the other PNNL staff members who interface with BES are also effective. Overall, though PNNL is the $5^{\text {th }}$ largest Lab he works with, Walt rated the strength of PNNL programs very high and the quality outstanding.

BES is happy with the level of service provided by PNSO. Walt noted a need for BES to communicate more with PNSO about BES programs.

Finally, ASCR Associate Director Ed Oliver commented that PNNL has good, well managed ASCR programs, but there aren't enough of them. He is impressed that the supercomputer (HPCS2) is up and running with a Linpack benchmark completed. It's too soon to judge the degree to which we will be able to demonstrate its usefulness for the computational science we do here, but Ed believes there's potential.

Ed expressed satisfaction with the support from the PNSO, indicating that overall ASCR's interface with PNNL and PNSO are among the best of the labs for the work we do for ASCR.

\section{Directorate Review Committee (DRC)}

The committee applauded the organizational changes begun a year ago, affirming that the overall organization of FSD was greatly strengthened by these changes.

The committee found the chemical sciences program very strong, world-class in fact, and encouraged us to continue our push for a major investment in catalysis that could manifest itself in a center or facility. They noted the need to re-invigorate the computational chemistry program after the loss of talent during the past year.
The committee praised the EMSL organizational changes and the implementation of the new user model. They saw significant progress in redirecting the user program to meet BER's expectations.

The committee regarded the atmospheric sciences and climate research effort to be world-class and commented favorably on the proposed climate physics program as a thrust whose time has come, and which represents a wonderful opportunity for PNNL and DOE.

The committee was pleased with the status of the biology program, and the consensus is we are a very strong contender for one of the GTL facilities. DRC member Ken Nealson called PNNL "the place to be" in microbial systems biology. We are encouraged to continue to grow relationships with our scientific peers, and to continue to strengthen the links between chemistry and biology. We were applauded for setting up a pilot high-throughput proteomics capability without interfering with our highfield mass spectrometry work.

Committee members commented favorably on the presentation of work related to uncertainty in modeling and simulation, while also pointing to the need to recruit leadership into the computational science and mathematics organization.

The committee encouraged us to clearly articulate our scientific objectives and to not get sidetracked from this by the necessary attention to growth in sales and business volume.

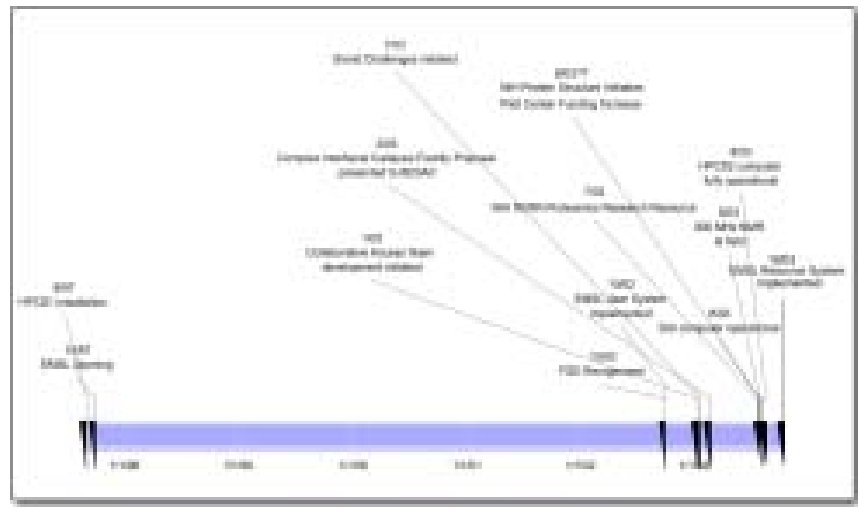

Table 1.1.1. FY2003, a watershed year, marks the beginning of a more proactive and targeted approach to accomplishing DOE's science mission 


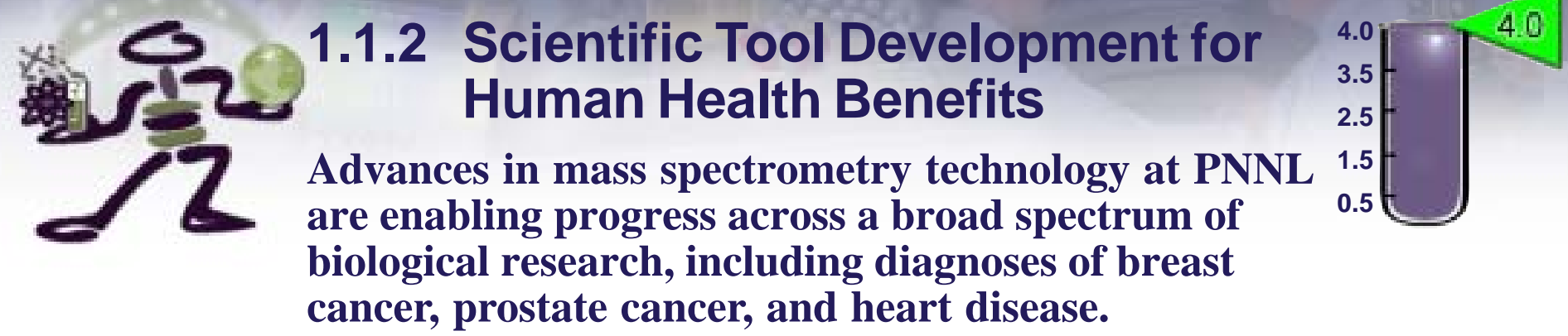

With DOE-BER support, PNNL established a highthroughput proteome analysis pilot production line that is expected to serve as a precursor for additional lines planned for FY2004. This development effort sets the stage for PNNL to compete for the GTL whole proteome analysis facility. Developing these capabilities will lead to continued advances across an expanding spectrum of research areas such as those highlighted below.

\section{Breast Fluid Analysis Shows Promise for Cancer Diagnosis}

A new method of extracting and analyzing fluid from a woman's breast may provide a more accurate, less expensive, and noninvasive way to determine her risk for breast cancer or to diagnose the disease in its early stages.

In a paper published in the July 3 issue of Breast Cancer Research and Treatment, PNNL scientists and research colleagues at University of California, Los Angeles report they have discovered six times more proteins than previously identified in nipple aspirate fluid (NAF). The presence of such proteins suggests that NAF, which is extracted using a breast pump, could be a resource for biomarkers, or biological indicators, of breast cancer, which is expected to claim the lives of approximately 40,000 American women this year.

"We believe this fluid could be the best alternative for discovering biomarkers for early-stage breast cancer," said Rick Zangar, the PNNL principal investigator. "With further analysis, we could detect up to 10 times more proteins in NAF. The more proteins we identify, the better chance there is to find one that is linked to breast cancer."

Proteins can serve as biomarkers of disease. When cancerous cells begin to develop, they create their own proteins that, if detected in NAF, could indicate the presence or risk of breast cancer. Current methods for screening include breast self-exams and mammograms, which are physical exams compared with the molecular approach at PNNL.
"NAF offers a unique window through which we can monitor the processes occurring inside the breast ductal system," Zangar said. "The medical community recognizes that one way to markedly decrease mortality from breast cancer would be a more accurate, noninvasive method for early diagnosis. Our approach suggests there could be other options available."

Zangar and colleague Susan Varnum analyzed NAF samples using EMSL high-throughput mass spectrometers. Of the 65 proteins identified, 15 of them, or $23 \%$, have been previously implicated as potential biomarkers for breast cancer. This implies that NAF provides a useful sample for analysis of known breast cancer biomarkers, while offering the possibility of providing novel biomarkers not yet identified in blood samples. The Department of Defense Breast Cancer Research Program funded the majority of the NAF research.

\section{Blood Serum Protein Library}

Plasma proteins provide a wealth of diagnostic tools. For examples, physicians look at high blood concentrations of a protein called prostate-specific antigen as an indication of prostate cancer.

Scientists have previously identified a few hundred of the estimated thousands of proteins in a person's bloodstream, but there may be many more. Between 1977 and 2001, scientists screening human plasma for new proteins added only about 20 proteins to the tally.

The search for plasma proteins is speeding up. PNNL developed a method involving preprocessing steps to create blood serum from plasma, use of antibody binding to remove most immune proteins, and dividing of proteins into peptides with enzymes.

A series of chromatography techniques was then used to disperse the peptides in a liquid or gas environment. Then each group of peptides was injected into a tandem mass spectrometer from which the researchers deduced the blood proteins. 
The team applied this procedure to plasma from a healthy woman and identified 490 proteins, more than twice as many as identified by other techniques. Clearly, application of high-throughput mass spectrometry technology at PNNL has enormous potential to rapidly accelerate protein identification in blood serum, which should ultimately prove valuable in disease diagnosis. This work is a first step in compiling a master library of proteins in plasma.

A paper titled "Toward a Human Blood Serum Proteome" by Joshua Adkins, Susan Varnum, Kenneth Auberry, Ronald Moore, Nicolas Angell, Dick Smith, David Springer, Joel Pounds, was published in the Journal of Molecular \& Cellular Proteomics (online at http:/ /www.mcponline.org/cgi/content/abstract/1/12/947). It received additional exposure in Science News (Vol. 163, No. 11 - http://www.sciencenews.org/20030315/ bob9.asp ), including being featured on the cover page.

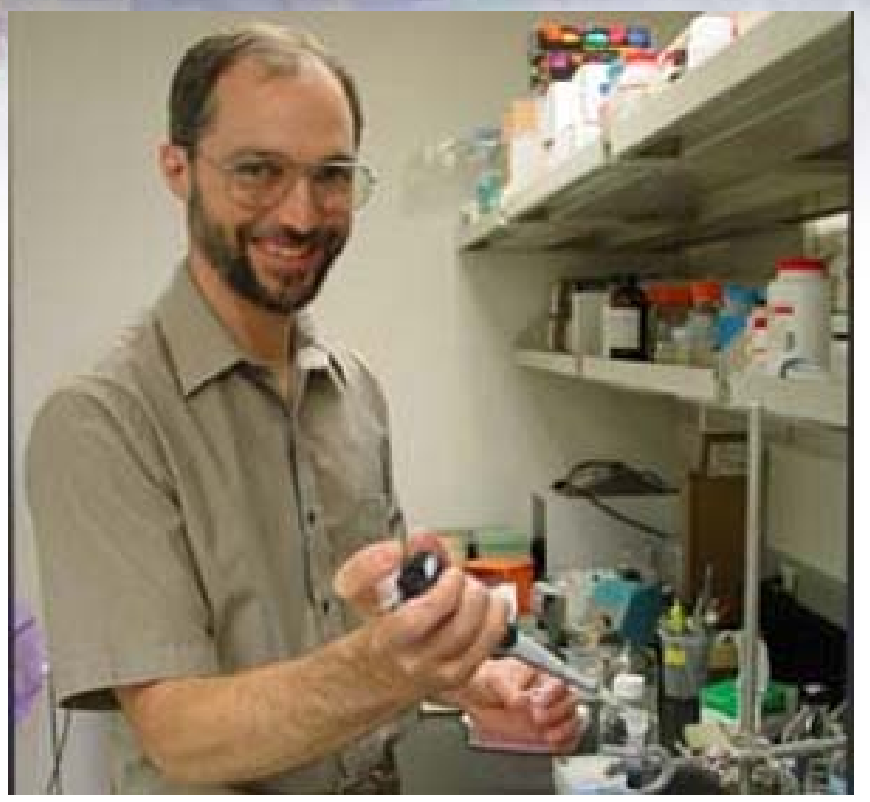

Figure 1.1.2. PNNL scientist Rick Zangar is researching new techniques to detect breast cancer. 


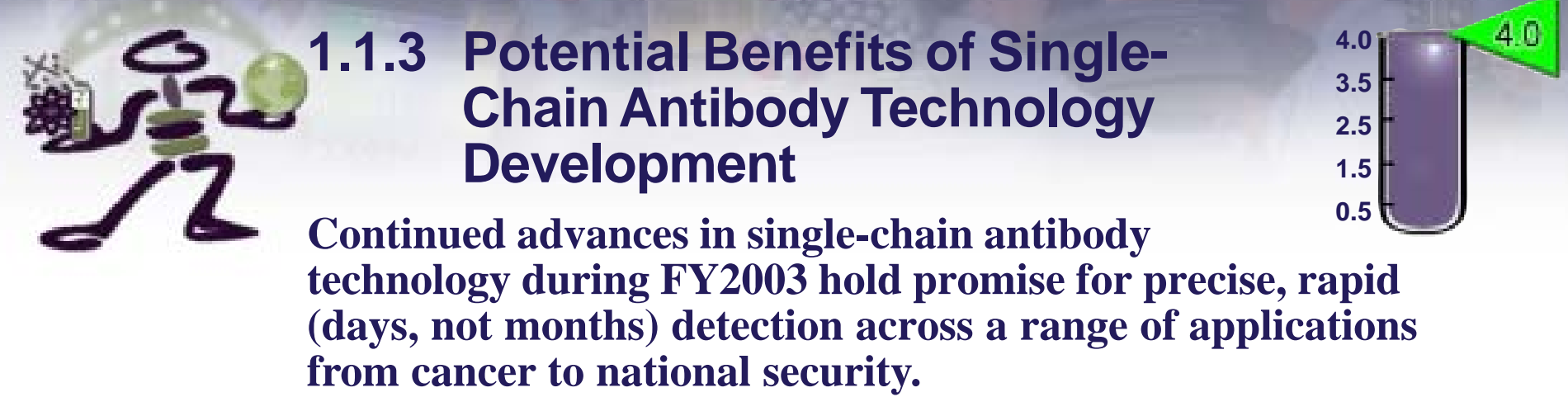

\section{Rapid Identification of New Antibodies}

Antibodies are proteins produced by white blood cells as part of the immune response. Scientists at PNNL have extracted part of the human immune system and reconstituted it in brewer's yeast in a way that enables powerful machines to quickly identify new antibodies. The advance could have major implications for fundamental biological science as well as for industries that use antibodies for sensors, biodetectors, diagnostic tools, and therapeutic agents.

The technology could replace the need to produce antibodies within animals, such as mice, and opens up new possibilities for rapidly designing medical treatments more acceptable to the human immune system.

"Our antibody library offers many advantages over traditional approaches. We expect it will be a more effective tool for scientists," said Michael Feldhaus, PNNL pathologist and microbiologist and lead author of a paper that appeared in the February issue of Nature Biotechnology $y^{(a)}$. "Regulated expression of these antibodies allows the library to be expanded while maintaining its diversity. Furthermore, our unique identification process means we can screen for antibodies in days rather than the months it may take using other approaches."

Feldhaus and colleague Robert Siegel, a PNNL microbiologist, built a library of 1 billion human antibodies and expressed them on the surface of yeast cells using a platform designed by collaborator Dane Wittrup of the Massachusetts Institute of Technology. The combined technologies offer a more powerful, less-expensive method for identifying antibodies.
Antibodies play an increasingly important role in industry because they are effective tools for recognizing specific molecules. When antibodies bind to a specific protein on bacteria, it signals other cells to either kill or remove the bacteria. In medical treatments, antibodies are being injected into the body to seek out specific proteins on cancerous cells, for example, and target treatment to those cells. Biowarfare detectors can use antibodies to locate proteins as a way of identifying harmful agents. Antibodies also are expected to play a major role in helping scientists more fully understand various biological processes by identifying which proteins are present and if they interact with any other proteins in the cell.

Most importantly, by incorporating Wittrup's yeast surface display method, PNNL scientists can readily modify how an antibody binds to proteins. Being able to increase how tightly a protein and antibody bind together could, for example, increase antibody effectiveness for detecting pathogens or disease.

The library developed at PNNL identifies antibodies more quickly than previous methods, thus reducing labor costs. To accelerate the identification process, PNNL combined two types of cell sorters - high-throughput parallel magnetic cell sorting and high-resolution linear flow cytometric cell sorting — to isolate specific antibodies very quickly.

Wittrup originally developed the yeast surface display as a way to improve the binding of antibodies to chemicals while working at the University of Illinois in the late 1990s. Now, he uses PNNL's antibody library with his display platform in a multitude of studies, many directed at development of novel cancer therapeutics.

Says Wittrup, "This yeast library provides a powerful and direct route to the in vitro isolation of useful antibodies,

(a) Feldhaus, MJ, RW Siegel, LK Opresko, JM Weaver-Feldhaus, YA Yeung, JR Cochran, P Heinzelman, D Colby, J Swers, C Graff, HS Wiley, KD Wittrup. 2003. "Flow cytometric isolation of human antibodies from a nonimmune Saccharomyces cerevisiae surface display library.” Nature Biotech. 21:163-170. 
and is a complementary approach to analogous alternatives such as phage display and ribosome display. We expect the wide availability of this library will open a door into antibody engineering technology for life sciences researchers currently using classic mouse hybridoma methods to make affinity reagents. PNNL should significantly impact the research community through the broad distribution of this library."
PNNL has received additional funding from the Department of Energy to apply the antibody library to bioterrorism detection. This research was conducted with funding from the National Science Foundation, the Hereditary Disease Foundation, and internal research support from PNNL's Biomolecular Systems Initiative.

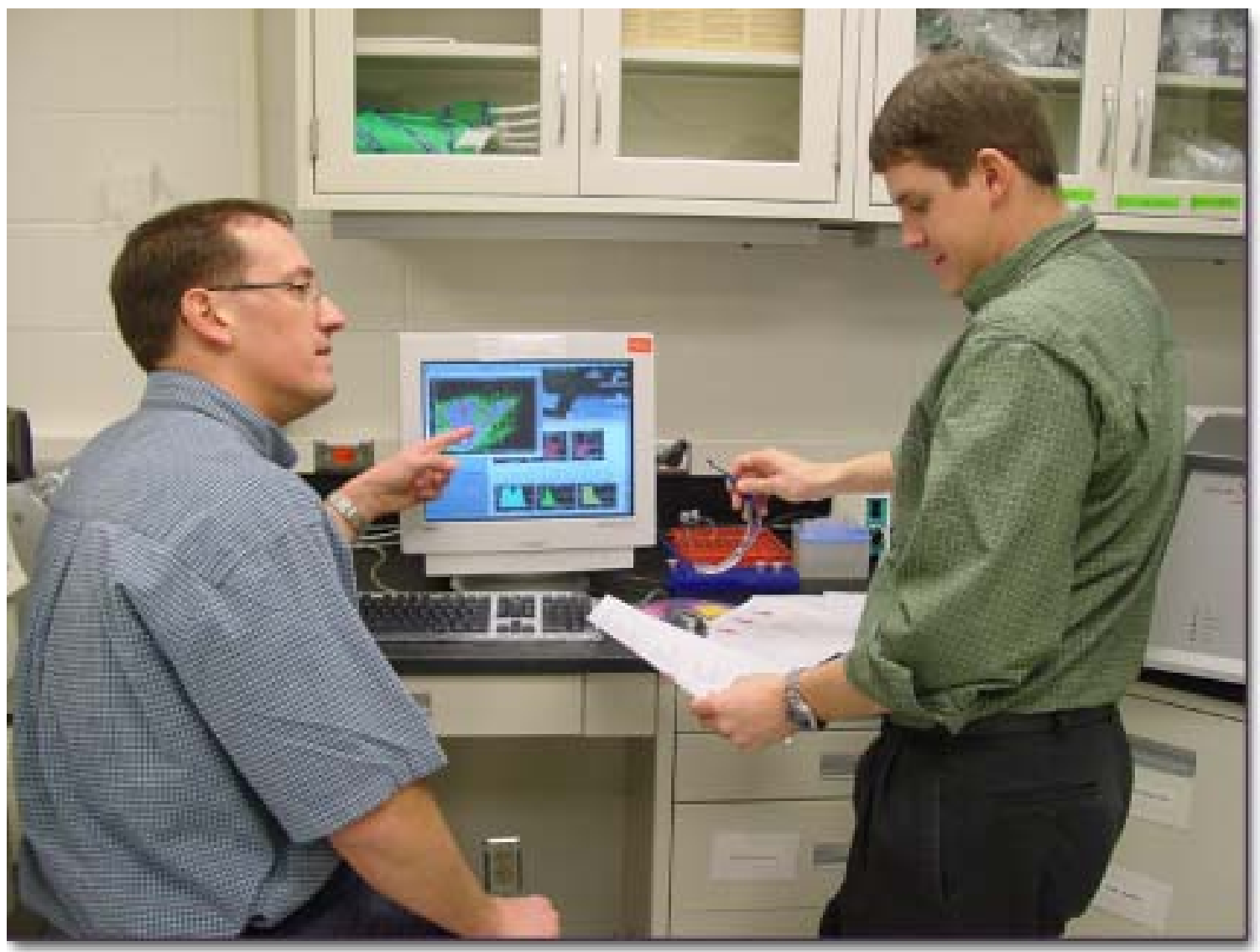

Figure 1.1.3. PNNL scientists Michael Felhaus (seated) and Rob Siegel (standing) developed a library of 1 billion human antibodies on brewer's yeast in collaboration with MIT professor Dane Wittup. Using high-speed flow cytometers to generate synthetic antibodies, they eliminate the need to use live animals. Synthetic antibodies and other technologies under development at the Prototype Sample Processing and Proteomics Facility will provide tools for tackling a broad range of important biological problems. 


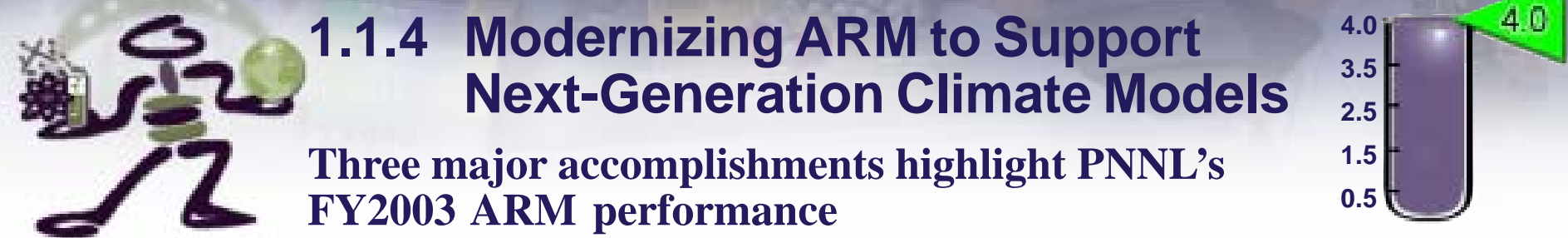

\section{ARM Data System Modernization Completed}

Since the beginning of ARM, PNNL has been in a key leadership and engineering development role for the data system linking ARM field sites and the various ARM functional activities. In 2002 and 2003, the ARM Engineering and Operations teams collaborated closely on an extensive upgrade and modernization effort that has improved the performance, maintainability, and security of ARM's computing environment while improving the available communication bandwidth between field sites in Oklahoma, Alaska, Australia, New Guinea, and Nauru, and the key processing site at PNNL. Key elements of the upgrade included broadband satellite connections to remote sites, common hardware, software, and networking, centralized processing at PNNL of hourly streamed data, centralized data QA and feedback to sites, continuous visualization of data streams accessible from any location, and improved network and system security.

\section{Remote Sensing Techniques Determine Properties of Mixed- Phase Cloud Properties}

PNNL Scientist Dave Turner and colleagues developed a technique for determining cloud hydrometeor phase (i.e., whether a cloud contains liquid droplets, ice particles, or both) and effective size for the water and ice particles in polar clouds. This is a climatologically important class of clouds that has been ignored because of the difficulty in determining these properties when particles of differing phase exist in the same spatial volume. The new technique uses ground-based, high spectral resolution infrared observations. Cloud phase is determined by using data from a spectral region where the ice is more absorbing than liquid and a spectral region where the opposite is true. Observations in the latter region can only be made when the atmosphere contains relatively small amounts of water vapor, such as in the polar regions of the globe; however, the Arctic experiences mixed-phase clouds approximately $40 \%$ of the time. The new PNNL algorithm is well suited to study clouds in this environment. The technique has been applied to seven months of data collected at the Surface Heat Budget of the Arctic Ocean (SHEBA) experiment, which was supported by the Department of Energy's Atmospheric Radiation Measurement (ARM). The analysis of these data is providing new insights to Arctic clouds, their physical properties, and their effects on the Earth's climate.

\section{New Approaches to Representing Clouds in Climate Models}

Since the 1980s, representation of cloud processes and the effects of clouds on the Earth's radiative energy balance have been recognized as the principal sources of uncertainty in climate models. Clouds are extremely difficult phenomena to represent in atmospheric models because their physics is complex - involving turbulent fluid dynamics; sensible, latent, and radiative heat transfer; mass transfer; hydrometeor nucleation and growth; multiple phases; precipitation; etc. - and it takes place on scales much smaller that those resolved by the models. Current climate models parameterize these phenomena via empirical/statistical relationships that relate their gross large-scale effects to the mean dynamic and thermodynamic fields resolved by the models. These parameterizations do a good job in representing many large-scale cloud effects, but they have problems, and these problems become more important as we ask more detailed questions about the effects of climate change on the kinds of spatial scales that matter to policy and decision makers.

To improve the representation of clouds and climate models and to test the hypothesis that the treatment of clouds is the source of major discrepancies among models, we must develop new parameterization approaches that represent cloud effects with more detail and more realistically. One possible new approach is called superparameterization. In this approach, conventional cloud parameterizations are replaced by a set of coupled 2-dimensional cloud resolving modes within each model grid cell. This approach greatly increases the amount of computer resources required to run the model, but it greatly improves its physical realism with respect to clouds. Such a model could be an important benchmark with which to test and improve more operational climate simulation models. 
PNNL scientists have begun to test whether or not a superparameterization model does indeed result in improved representation of key climate physics processes. In one such test, we compared predictions of surface radiative heat flux by a conventional and a superparameterized climate model with observations from the ARM site at Nauru, an island in the tropical western Pacific. Preliminary results from these comparisons, shown below, indicate that superparameterization does improve simulation of the surface radiative heat flux. Much more work needs to be done to fully explore the viability of the superparameterization approach (note that not all discrepancies between the observed and simulated fluxes are eliminated). But these results offer encouragement that new approaches for treating clouds in models are feasible and that these approaches may resolve certain model performance problems that have been troubling climate modelers for more than a decade.

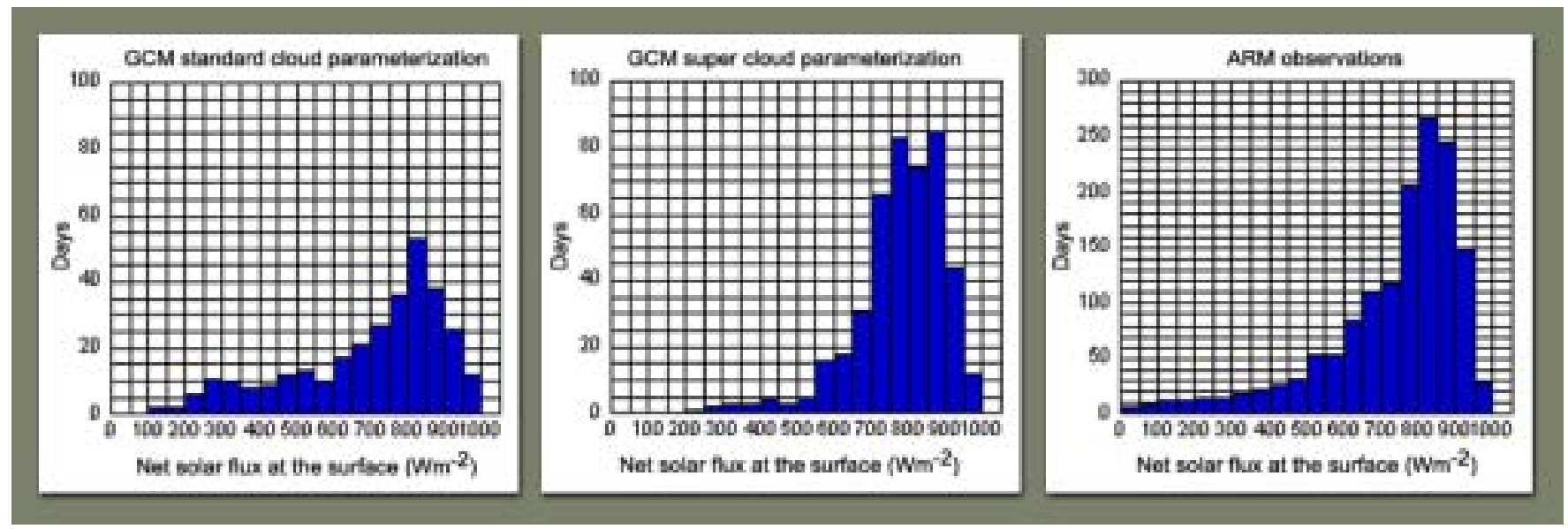

Figure 1.1.4. ARM helps test hypotheses related to the accuracy of different cloud parameterization approaches. 


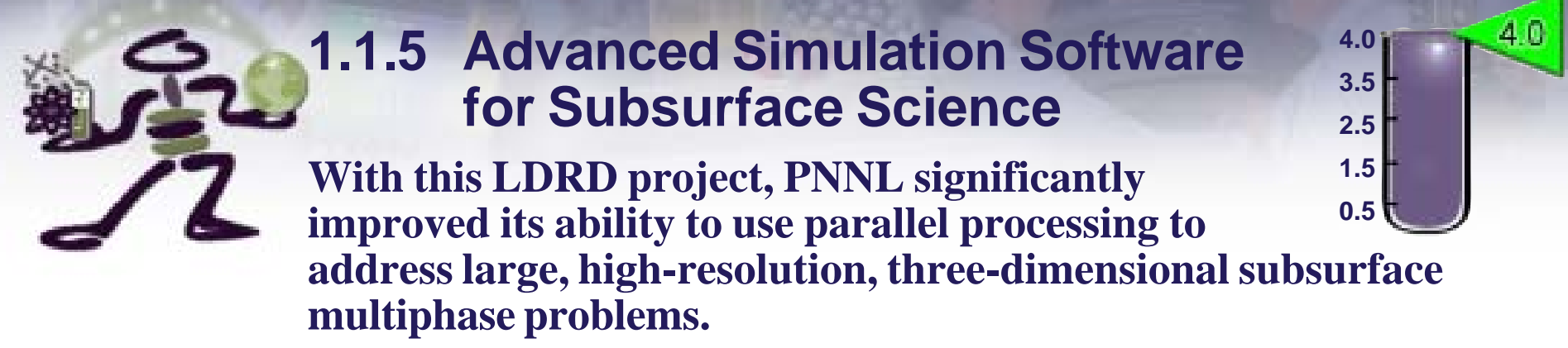

\section{The Need for Better Software}

Reliable predictions of field-scale subsurface behavior are essential to appropriate decision making related to cleanup of DOE's nuclear waste sites. The inherent complexity of the subsurface, coupled with a limited ability to observe processes and interactions as they occur, has proven to be a formidable obstacle to assessing risk and devising remedies. Computer simulation of fieldscale contaminant behavior that accurately represents the multiple physical and chemical processes operating on complex mixtures in heterogeneous subsurface materials is the critical factor for developing efficient cost-effective engineering strategies. Large massively parallel computers give us the hardware to attack this problem, but software and solution techniques need further development and verification.

\section{The Project}

During FY2003, PNNL made significant strides and set the stage for more advances in FY2004 through an LDRD project funded under the Computational Science and Technology Initiative. The work described here, which used PNNL's Subsurface Transport over Multiple Phases (STOMP) simulator software, is one example of the project's several accomplishments. The project targeted the migration of the 600 to 900 metric tonnes of carbon tetrachloride that have been discharged to surface cribs in the 200 West Area of the Hanford Site. Carbon tetrachloride is one of a class of liquids known as nonaqueous phase liquids (NAPLs) that behave much differently than aqueous liquids as they migrate through the subsurface. Progress was made on several fronts, including

1. Incorporating advanced parallel programming tools, utilities, and interfaces into subsurface simulation software to increase usability, computational performance, and efficiency. The STOMP90 framework, the sequential code being used to model the historical migration of carbon tetrachloride on the Hanford site, was converted to scalable form for execution on distributed-shared memory parallel computers.

2. Modeling a new constitutive theory for the migration of spreading and nonspreading NAPLs through the vadose zone that explicitly accounts for the formation of disconnected residual as well as mobile and entrapped NAPL; and

3. Validating the modeling experimentally.

\section{Success}

A one-dimensional test problem involving the migration of carbon tetrachloride through Hanford soils was successfully completed using the parallel implementation of the STOMP simulator on the Molecular Science Computing Facility Supercomputer. A three-dimensional test problem developed under the Hanford Science and Technology project was used for the final verification.

As part of this project, we developed new capabilities for the parallel Fortran Preprocessor, a distinctive PNNL resource that uses directives to generate parallel code from a standard Fortran-90 source. The successful port of the Fortran Preprocessor from the old Molecular Science Computing Facility IBM SP system to the new 64-bit Itanium-2 architecture, Hewlett Packard Linux operating system, and Intel Fortran-90 compiler, enabled the high-level physics coding in the STOMP simulator to be accommodated without modification.

The Fortran Preprocessor was the subject of a white paper, "Beyond MPI: A Need for High Performance Abstractions," that Matt Rosing and Steve Yabusaki were invited to present to the Programming Environments and Tools session of the National Coordination Office for Information Technology Research and Development (NITRD) Workshop on the Road Map for the Revitalization of High End Computing, June 16-18, 2003, Washington, D.C. 
The new constitutive theory for the migration of NAPLs and its incorporation into STOMP is the subject of a journal article submission: White, M. D., M. Oostrom, and R. J. Lenhard. 2003. "A practical model for mobile, residual and trapped NAPL in porous media." Ground Water (in review), May 2003. The model is currently being applied to the reconstruction of the historical migration of carbon tetrachloride in variably saturated vadose zone sediments, disposed in the 216 Z-9 and 216 Z-1a cribs on the Hanford Site. Mark White included these modeling results in an invited presentation to the DOE Computational Graduate Fellowship Conference, July 14-17, 2003, Washington, D.C., "Crossing the Scales of Subsurface Science via Parallel Computing."

This version of the simulator will allow numerical simulations of carbon tetrachloride migration to be conducted at greater resolution, in larger domains, and with decreased execution time. With such a tool, we can design robust, large-scale subsurface NAPL remediations.

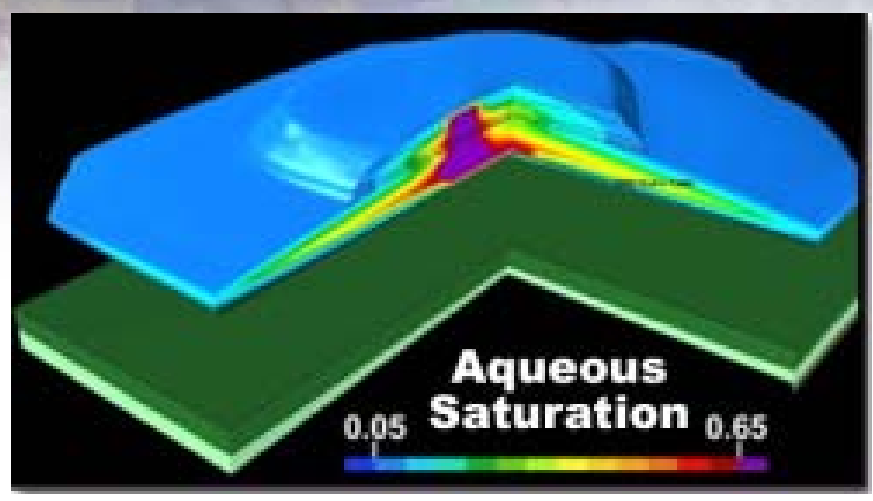

Figure 1.1.5. A new constitutive theory for the migration of spreading and nonspreading nonaqueous phase liquids (NAPLs) through the vadose zone was incorporated into the STOMP multifluid simulator and successfully validated. The model is currently being used to reconstruct the historical migration of carbon tetrachloride in variably saturated vadose zone sediments on the Hanford Site (White 2003). 


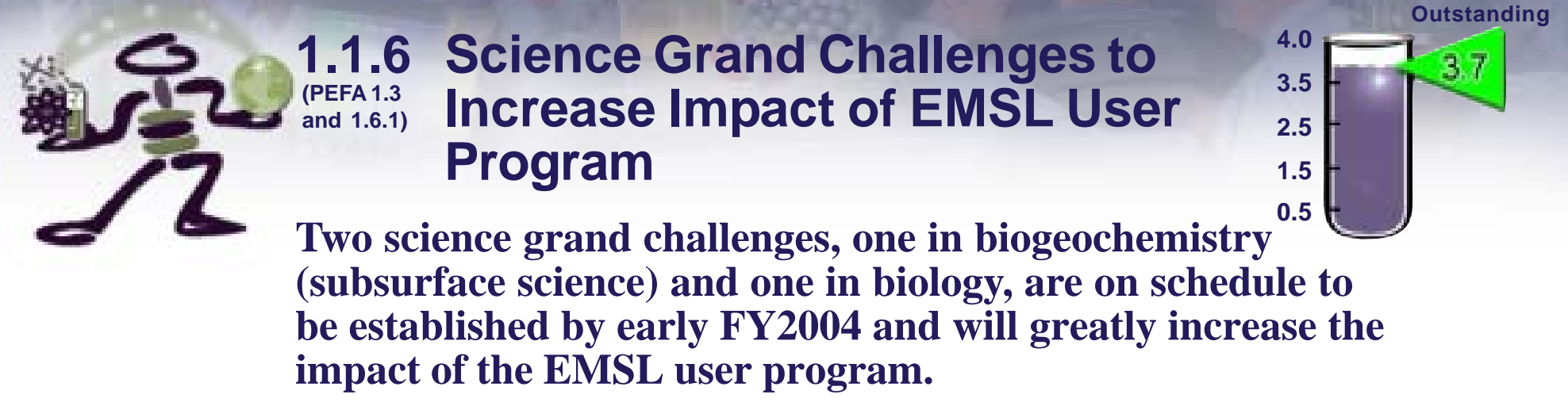

The scientific grand challenges are designed to leverage integrated sets of EMSL research capabilities in the study of highly significant scientific problems. The grand challenges, which will last three to five years, will create new benchmarks for integrated research. These challenges will be aligned with DOE mission areas; will be driven by users; and will take full advantage of EMSL's unique capabilities, resources, and technical expertise. These scientific challenges are designed to attract and involve users who are among the best scientists in the world in the area of the challenge and will greatly increase the impact of the EMSL user program.

During FY2003,

- Working closely with OBER and the EMSL User Advisory Committee, EMSL scientist drafted a plan for developing the grand challenges. After a lengthy negotiation, the plan was approved by BER in September 2003.

- Both grand challenge steering committees - recognized authorities in the two theme areas -met in FY2003.

- For the BioGeochemistry Grand Challenge (BGGC), a workshop will be held at EMSL November 4-6, 2003. to develop a scope for a BER call for proposals. The Biology Grand Challenge (BGC) steering committee asked that a call for external "concept papers" be issued before scheduling a workshop. The call is to be finalized at meeting of BER staff and EMSL management October 23, 2003; the workshop will be scheduled once proposals are received.
BioGeochemistry Grand Challenge (BGGC): The tentative theme for the BGGC is mechanisms of microbemineral electron transfer mechanisms. The microbemineral interface is complex, important, and relatively unexplored. The molecular-scale mechanistics and linkages across this complex interface are poorly characterized, and the science involved spans broad fields in biology and the physical sciences. Important scientific questions at the base of this theme include the following:

- What is the structure and molecular architecture of the bacterial cell envelope-mineral surface region?

- What molecular interactions/reactions occur within this region to regulate electron flux to and from microorganisms?

- How do microorganisms sense and respond to physical and chemical changes that occur at mineral surfaces?

The processes to be studied under the BGGC are fundamental to DOE mission areas in energy, carbon management, and remediation. The significance is enormous, and the potential impacts far-reaching and the capabilities in EMSL's user program are well matched with the science to be investigated in this area.

Because of their ability to enzymatically reduce and precipitate a diverse range of heavy metals and radionuclides (see Figure 1.1.7), bacterial strains such as Shewanella oneidensis MR-1 are key organisms in the bioremediation of metals. Determining how electron transfer is facilitated at a microbe-mineral interface represents a major challenge and is the focus of EMSL's new scientific grand challenge in the bioGeochemistry. 
Biology Grand Challenge (BGC): The tentative theme for the BGC is membrane dynamics. Transport of information, material, and energy across membranes is important and complex. The molecular-scale mechanistics and linkages across this complex interface are not well understood, and the science involved spans broad fields in the physical sciences and biology. Important scientific questions at the base of this theme include the following:

- How do membrane protein complexes transduce information or energy across a membrane? Areas of interest include photosynthesis, mitochondrial respiration, and two-component signaling systems.

- Can the dynamics be simulated in a meaningful way?

- What are the constraints in the system?

- What are the structure-function relationships?

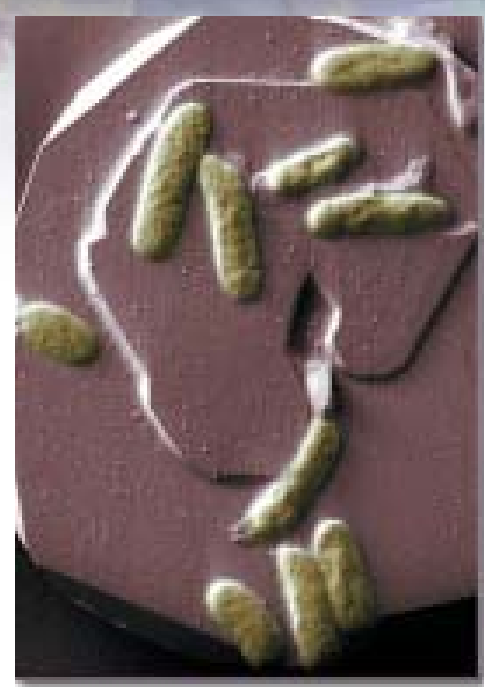

Figure 1.1.6. The electron micrograph shows Shewanella interacting with a singlecrystalline plane of a mineral surface. 


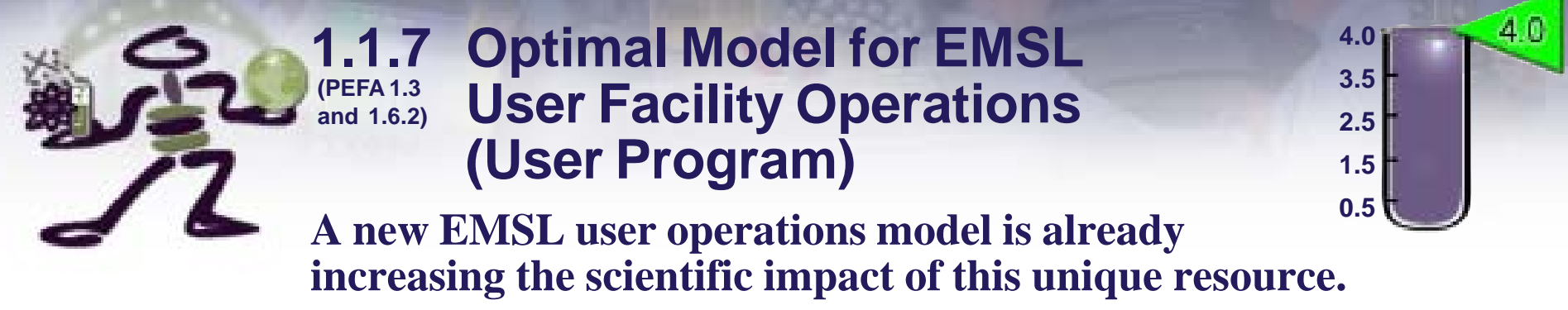

\section{New EMSL User Operations Model Now Operational}

In late FY2002, EMSL staff visited several user facilities to identify best practices in preparation for revamping the operation of EMSL. After evaluating the selected facilities, PNNL, in close coordination with OBER, the EMSL User Advisory Committee, and PNSO, developed a model combining the best features of each. Submitted to OBER and BERAC in FY2003, the model became the basis for a detailed EMSL Facility Operations Manual.

EMSL's plan embraces and supports the Office of Science's fundamental mission to advance basic research and develop the tools of science that comprise the foundations for the applied missions of DOE and PNNL. The model emphasizes attracting high-level scientists working in areas EMSL is strategically targeting.

\section{Characteristics of EMSL User Operations Model}

The new operations model effectively promotes EMSL's signature characteristics:

1) Highly competent teams developing enhanced research tools and methodologies that advance EMSL capabilities;

2) Appropriate integration of theory, modeling, and simulation with experimental programs; and

3) A collaborative mode of operation leading to joint publications and enhancement of research programs at user institutions as well as at the EMSL, effectively leveraging DOE's investment in this facility.

Elements of the operations model include a new advisory group-Science Advisory Committee-which, together with the (independent) User Advisory Committee, provides objective, timely advice to EMSL leadership.

\section{Collaborative Access Teams (CATs)}

Under the new operations model, users may access EMSL resources through the normal peer-reviewed proposal system or by participating in CATs or EMSL Grand Challenges.
CATs are comprised of teams of scientists nationwide, who use EMSL facilities and capabilities to conduct funded programmatic research around focused science themes. Membership in a CAT is open to all members of the scientific community who can contribute significantly to the CAT (e.g., funding, equipment, design and engineering, operations manpower, etc.).

Collaborative access teams enable and conduct highimpact science that demonstrates EMSL's capabilities and value, keeping at the forefront of science. CATs attract and thereby increase the number of high-impact users in a focused research environment and build new capabilities - for use by the CAT and everyone else as well.

\section{EMSL's Improved User Operations Environment}

This year's improvements in EMSL's operating philosophy and infrastructure result in more effective, efficient and user friendly operations by streamlining submission and evaluation of proposals to focus on those that best utilize EMSL's strengths and support its strategic objectives. The result is that higher quality proposals are accepted so that EMSL's resources are better focused on the most appropriate projects.

The EMSL user community continued to expand and grow. In FY2003, over 2400 scientists used EMSL capabilities. These users represent a broad diversity of scientific disciplines including chemistry, biology, environmental science and materials science. Additionally, there was diversity in the agencies that funded user research.

Productivity and tracking of user projects from the initial proposal to reporting of final results of supported research is facilitated by implementation of the new user system. This system greatly improves user publication capture through automated reminder emails and establishment of an EMSL coordinator to follow up and ensure that user publications are submitted to OSTI. Better tracking helps EMSL realize the scientific impact from each project. Module 1.1.10 provides additional detail on the user system.

Efficiency of the user survey process was improved significantly by implementing a web-based survey tool. From 
the 456 users who were contacted, roughly $21 \%$ responded to the survey. In rating the manner in which the EMSL environment facilitated scientific accomplishment, $95 \%$ were satisfied or very satisfied. Users acknowledged the excellent expertise, friendly attitude, and great equipment at EMSL. Responses to satisfaction with the availability of existing EMSL facilities and equipment prompted a $94 \%$ satisfied or very satisfied result. Respondents noted that EMSL staff worked closely with them to schedule access to instruments. When asked how satisfied users were with performance (e.g., were facilities and equipment maintained close to specifications), $95 \%$ of the respondents replied that they were satisfied or very satisfied. Of the respondents, $96 \%$ reported being satisfied or very satisfied with the support provided by EMSL staff and noted that their contacts were friendly, accommodating, and very competent. Seventy-nine percent of responders found the EMSL proposal system easy to use.

\section{EMSL Grand Challenges}

EMSL will implement two or more scientific Grand Challenges in the coming year. The primary purpose of these Grand Challenges will be to answer important scientific questions through a collaborative approach, using EMSL's capabilities. This approach will increase EMSL's and PNNL's scientific robustness and impact as the home of a user facility devoted to major scientific programs. These challenges will be consistent with DOE mission areas, focused on critical milestones in the advancement or use of science, will be user-driven, and will take full advantage of EMSL's unique capabilities, resources, and technical expertise to address significant problems. The scope of the scientific problems to be addressed will require multidisciplinary teaming, and their breadth will be of such magnitude that they cannot be addressed at any other single existing facility. The challenges will require not only the capabilities of EMSL but possibly other DOE user facilities as well as specialized instrumentation and capabilities at the participants' home institutions. The challenges will require significant management and coordination to meet their aggressive goals, and are expected to attract and involve users who are among the best scientists in the world. The challenges will generally have a set of well-defined goals in capability development or scientific impact and will have a specified duration.

\section{Increasing Science Impact}

EMSL has received nearly a dozen CAT proposals and will implement several in the next fiscal year. Researchers focusing on the Grand Challenges have identified two topics of national significance, and workshops are underway to

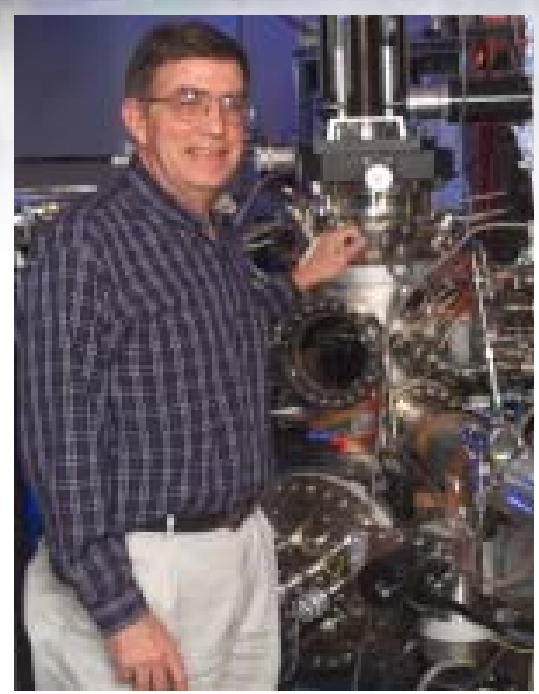

Figure 1.1.7a. Illustrating growing capabilities in catalysis, this year EMSL hosted J. Michael White from the University of Texas-Austin, who visited EMSL on a 9-month sabbatical. define specific topics to be addressed in biology and biogeochemistry. By refocusing of EMSL (and PNNL) resources, and targeted recruitment of users, EMSL is building a critical capability in these areas.

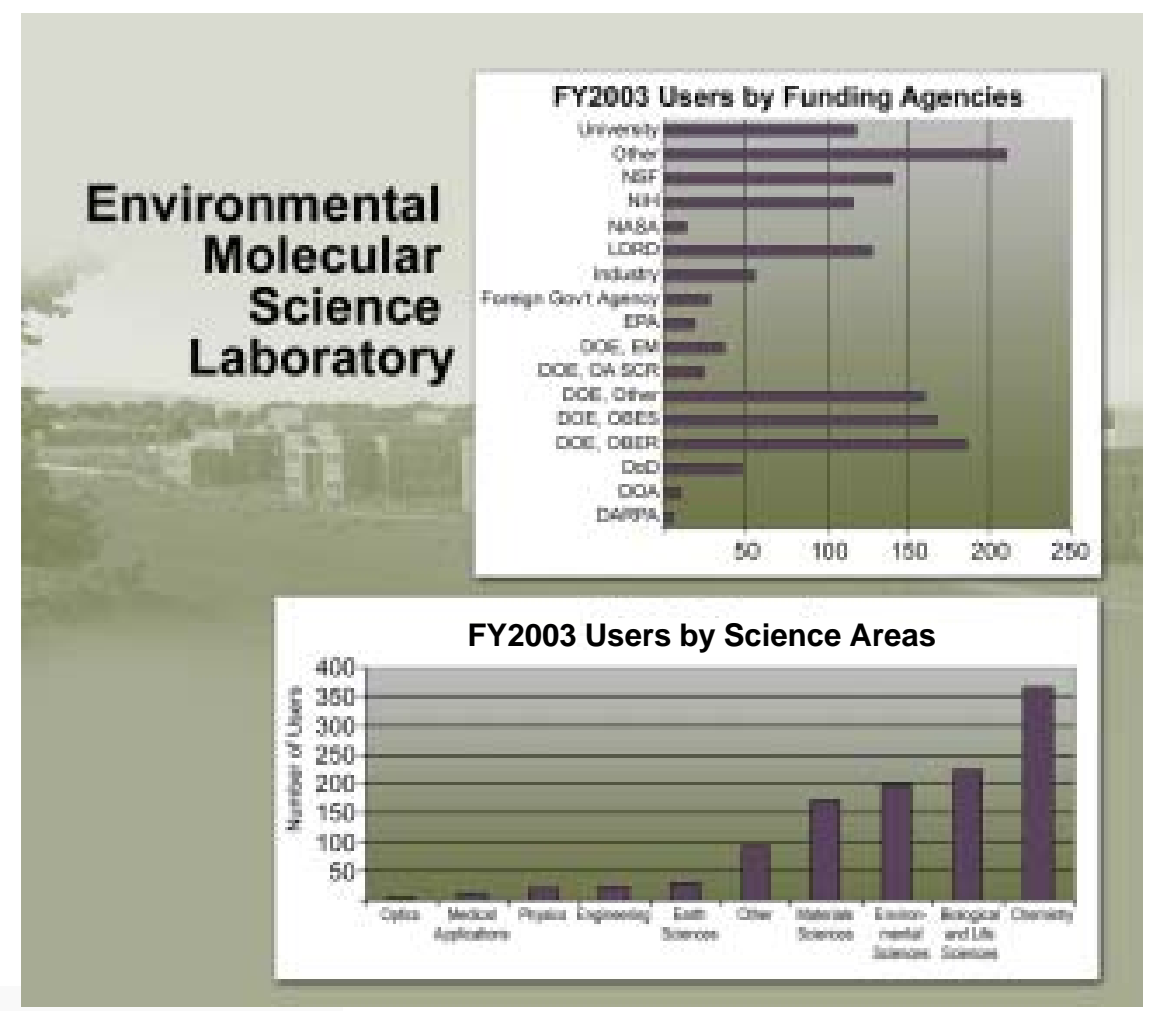

Figure 1.1.7b. The EMSL user community represents a broad diversity of scientific disciplines and agencies that funded user research. 


\subsubsection{Resolution of EMSL Magnet Concerns}

\section{FSD effectively managed concerns about safe operation of high field magnets in EMSL through immediate action and outside expert review of operations.}

In March, three incidents involving unplanned events with high field magnets in EMSL prompted concern about safety of operation and downtime. On Saturday, March 29,2003 , an accident resulting in an injury occurred when a staff member, who had removed a shield from the bore of the magnet, subsequently walked past the bore with an eight-ounce hammer in his hand. The hammer was forcefully pulled into the bore of the magnet, and with it came the back of the staff member's finger, which slammed against the side of the magnet causing blunt force trauma requiring 10 stitches to repair. (No permanent loss of finger movement resulted.) A week later, a different superconducting magnet spontaneously gave up energy (quenched) one evening. The event was reported as an explosion resulting in widespread disruption to the next day's lab activities. Nine days later, when the magnet was reenergized, a craftsman installing new engineering barriers thought the magnet was still deenergized. He brought his drill too close to it resulting in another powerful attraction, this time only involving metal.

Because these incidents happened so close to one another, PNNL conducted a comprehensive review of its magnet safety practices and made changes in the engineering protection and operating procedures. Recognizing that other opportunities to improve work procedures may have been missed, PNNL decided to solicit the advice of outside experts. To this end, a review board consisting of two internal and two external reviewers was convened onsite from July 7-10, 2003. The members of the team were:

- Dr. Harold R. Udseth, Staff Scientist, Macromolecular Structure and Dynamics, Biological Operations and Mass Spectrometry, PNNL, Review Team Leader

- Nicole M. Bernholc, CIH, Brookhaven National Laboratory, Safety and Health Services Division, Industrial Hygiene Group

- Dr. Paul D. Ellis, Laboratory Fellow, Macromolecular Structure and Dynamics, Fundamental Sciences, PNNL.
- Dr. Bruce L. Brandt, Director of DC User Facilities, National High Magnetic Field Laboratory, Florida State University.

\section{Assessment Report Results and Corrective Action}

The assessment report executive summary stated that, "PNNL magnet operations are certainly consistent with 'best-in-class'. In fact, they are exemplary. Strengths include the dedication of the people involved, the thoroughness of the Job Planning Process, the rapid, appropriate response when an incident shows the need for additional controls. The principal recommendations are that information about the hazards of superconducting magnets and the designs of engineered controls reflect the hazards of high magnetic fields and high magnetic field magnets in prioritized order reflecting the acuteness of the hazard. That order is pacemakers/defibrillators, loose ferromagnetic objects, metallic implants, other medical implants, magnetic tape/disks, credit cards, watches, prolonged exposure to high magnetic fields. That this information should be consolidated at a single point and that it be referenced from each document dealing with work around the magnets."

The review team noted that although all necessary information was present, access to it was unnecessarily complex, and it did not present the hazards of working around these magnets in an order reflecting the acuteness of the hazard. Specific recommendations included:

1. PNNL should review, and revise as appropriate, all documentation, signage, training, and engineered controls to ensure that the hazards of high magnetic fields and high magnetic field magnets are identified and controlled in prioritized order reflecting the acuteness of the hazards. Specifically, the hazard associated with flying metal objects equals the biomedical hazard for implants associated with magnetic fields and exceeds general exposure concerns, and should therefore be prominently and explicitly addressed. 
2. The team carefully considered the advisability of adding indicators, such as lights, that would show when the magnets were energized. This can be a valuable aid for driven magnets that are routinely energized and de-energized, but for superconducting magnets that are energized more than $99 \%$ of the time a better practice is to assume that the magnets are always energized. All documentation about high field magnets should indicate that they are assumed to be on unless clearly labeled otherwise.

3. Remove all documentation and labeling of DC magnetic fields from the Nonionizing Radiation category. DC magnetic fields do not radiate, they are tied to the magnet. The "radiation" category carries a lot of baggage that leads the development of safe work practices in inappropriate directions.

4. Documentation should be reviewed for consistency with PNNL policy of placing signs and demarcations of regions of high magnetic field, including noting the differences among horizontal, shielded, and unshielded magnets.
5. Consider developing a checklist to insure that consistent information is relayed during the hands-on portion of the training.

6. Heavy reliance on engineered controls in shared use rooms should be continued and emphasized in planning the layout of equipment, installation of barriers, and training of users for such rooms.

When final, the report will be submitted to EMSL management for action. Many of the recommendations have already been accomplished.

\section{Bottom Line}

These incidents drew rapid management response which resulted in improvement of our magnet operations, but also resulted in verification by outside experts of the quality of our overall magnet safety program. The response to these incidents is an example of effective R\&D management.

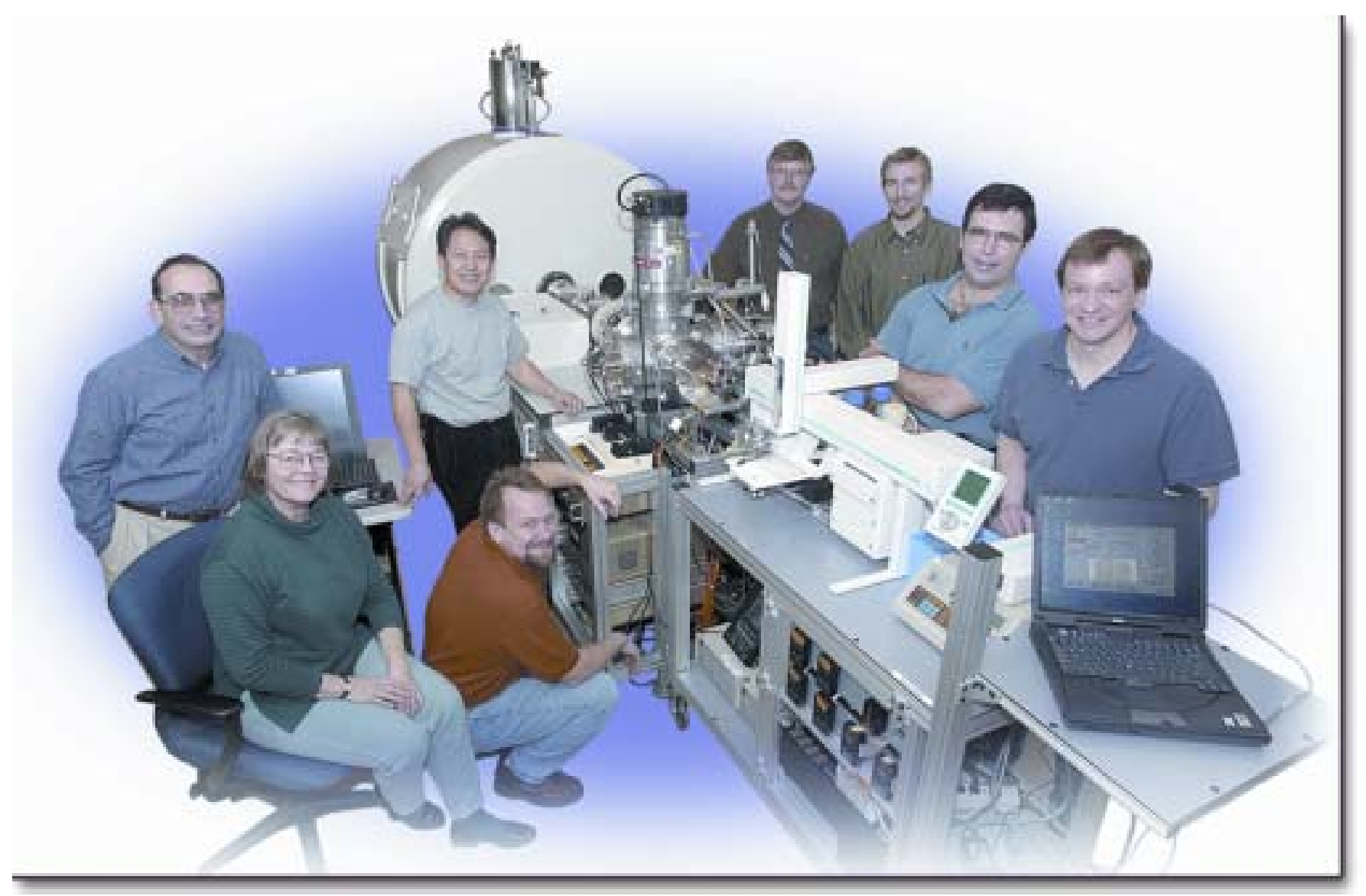

Figure 1.1.8. External assessment of magnet operations provided recommendations to improve safety near magnets like the one in the rear of this picture. 


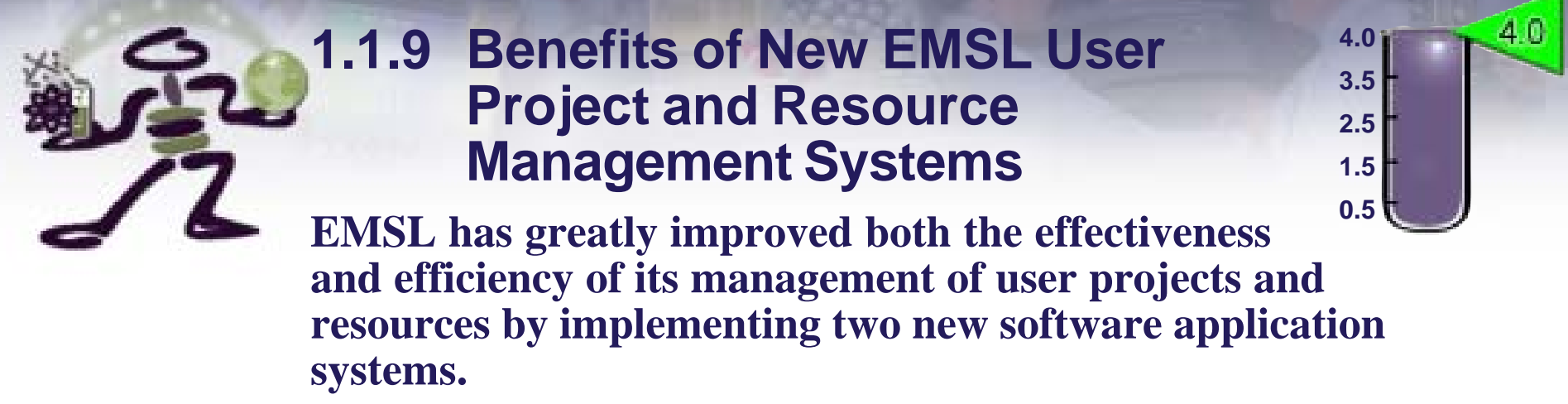

Each year, EMSL receives nearly 400 research proposals from scientists nationwide who wish to apply one or more of the facility's 50 state-of-the-art instruments to projects of fundamental research in areas such as biology, chemistry, atmospheric science, and groundwater transport. The EMSL panel that reviews proposal submittals considers the quality of research plans; the adequacy of attention to technical risks associated with the proposed research; whether use of personnel, facilities, and equipment is beneficial to the overall PNNL mission; and the potential success in identifying and avoiding or overcoming technical problems. Technical results following completion of the research are publicly communicated to maximize the value of the results and to gain appropriate recognition for DOE and PNNL.

In the past year, EMSL has greatly improved both the effectiveness and efficiency of its management of user projects and resources by developing and implementing two new software application systems:

- the EMSL User System (EUS), which facilitates receipt and processing of proposals and communications with users, and

- the EMSL Resource System (ERS), an internal product that tracks instrument use.

\section{EUS Project Management Tool}

Previously, user projects were not consistently tracked from proposal through result publication. This new webbased workflow tracking system facilitates user access to EMSL, from submittal of the initial research proposal to the tracking of publications resulting from each project. The EUS is multifaceted, consisting of an external website where users submit proposals electronically, and an internal application that allows EMSL staff to review and approve or deny proposals, monitor the status of a project, and close the project upon completion.
A reporting tool generates statistics. The EUS also interacts with PNNL's ERICA system, which submits scientific publications to DOE's Office of Scientific and Technical Information. The EUS shares general user information with ERICA; this enables staff to enter in ERICA those publications resulting from usage of EMSL, but where none of the authors are PNNL research staff.

\section{Highlights}

- Centralized, standard data collection and management.

- Quick and easy generation of reports, providing an ata-glance view of research at EMSL that can be used for strategic planning (e.g., allocation of resources, purchase of new equipment).

- Allows EMSL to better meet DOE requirements for tracking and reporting facility use.

- Faster and more convenient access to resources via an online proposal form; enables oversight of the proposal status by staff.

- Automatic e-mail notification of proposal status and requests for actions or information.

The EUS was fully implemented in EMSL in October 2002. Since then, it has significantly reduced the number of man-hours required to collect information and generate activity reports, and has improved EMSL's response time to proposals for scientific use of resources. EMSL staff received favorable feedback when demonstrating the EUS to DOE, other sponsors, and related institutions. As a result, several other national user facilities have expressed interest in porting the software application to their locations.

\section{ERS Instrument Usage Tracking Application}

The ERS was developed to track use of EMSL's major instruments and facilities, and was fully implemented in 
FY2003. The internet-based system - accessible from a desktop computer - allows reservations to be made using a drag-and-drop scheduling system. Reservations are displayed, by instrument, in a calendar-like grid and are color-coded according to usage type (e.g., programmatic, onsite, remote, etc). Each reservation can be linked in the database to an EMSL user proposal or identified with a PNNL staff member requesting usage.

\section{Highlights}

- Effective scheduling of resources is possible across multiple instruments.

- Reports are easily generated, showing instrument usage as well as the percentage of time that the machine is available for use.

\section{Cost-Effective Solutions}

PNNL developed the EUS and ERS applications using all open-source solutions, with the exception of a commercial reporting tool. The applications are platformand database-independent. The systems were developed on Linux and Windows systems and are operated on a Unix system. EMSL has selected the MySQL database, an open-source alternative to commercial databases that typically charge yearly license fees. The server used is JBoss, an open-source Java-based server. The JRport reporting tool, purchased from Jinfonet.com, is the only commercial product used.

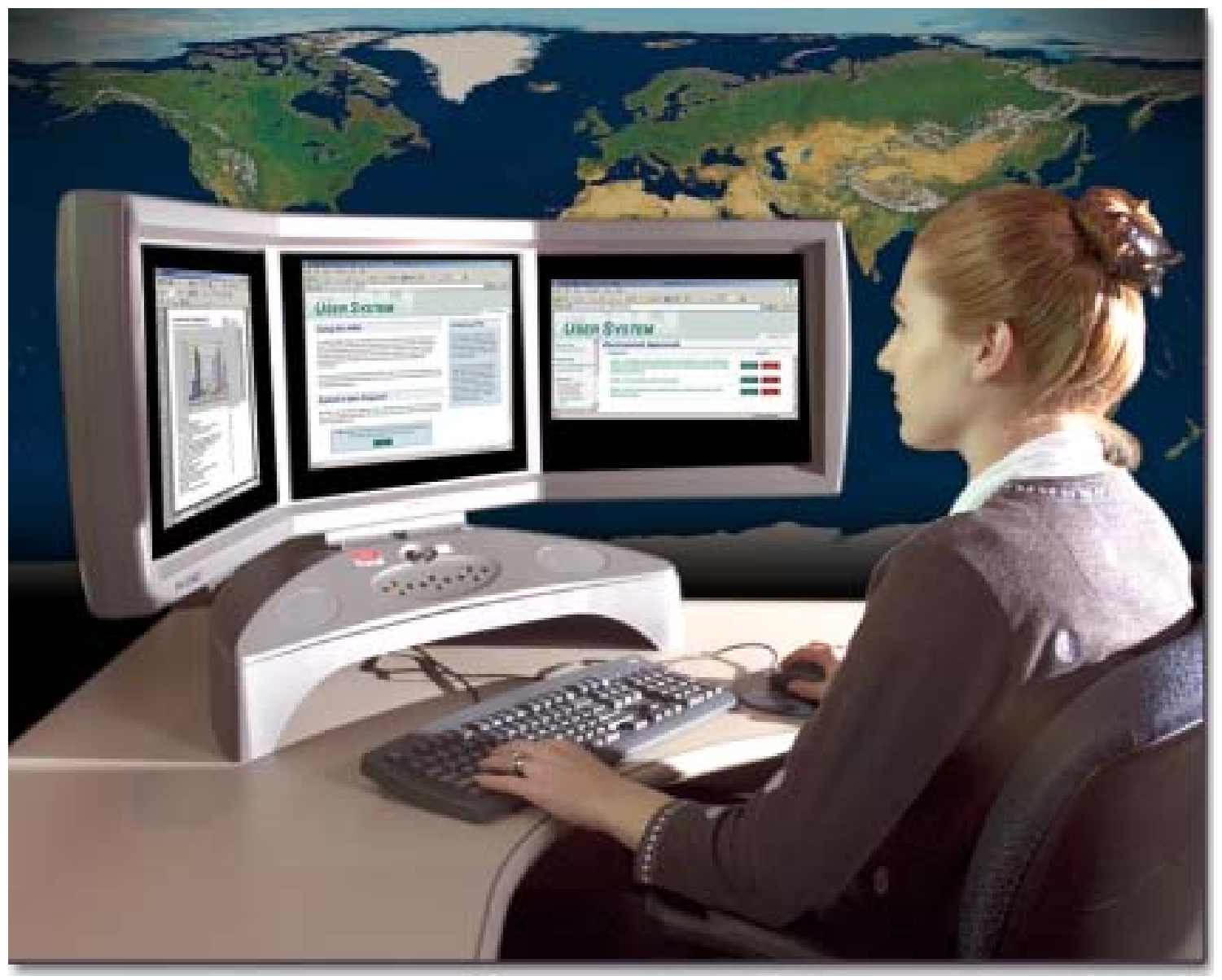

Figure 1.1.9. EMSL's software applications enable effective and efficient management of the Laboratory's resources. 


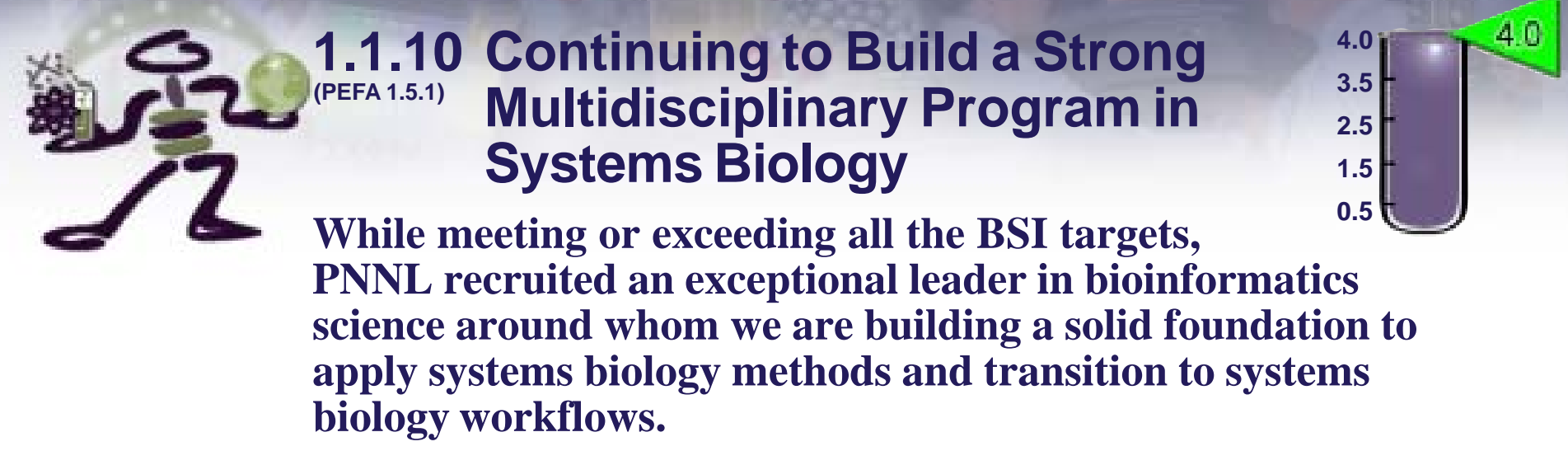

\section{Recruiting}

Biomolecular Systems Initiative (BSI) is exploiting the opportunity to build PNNL's bioinformatics capability around new lead scientist, George Michaels. His background in multiple computational and biological areas boosts the Lab's growing portfolio of bioinformatics and computational biology capabilities critical to GTL. Important aspects of PNNL's role in GTL lie in its capacity to 1) manage large-scale data archives for mass spectrometry (MS), expression, and image data; 2) build experimental workflow management systems; 3 ) create analysis toolkits, new algorithms, and analysis pipelines; and 4) build modeling and simulation tools and technologies, community databases, and fully developed computing hardware and network infrastructure.

Other recruiting successes in FY2003 include 1) Vladimir Kery, molecular biologist senior hire, who brings considerable expertise in high-throughput proteomics processing; 2) Ron Taylor, bioinformatics senior hire from the University of Colorado Health Sciences Center, who offers in-depth computational biology experience; 3) three mid-level cell biologists and microbiologists with excellent backgrounds. Among these, Hans Scholten is one of only two microbiologists available in the nation with expertise in syntrophy, bioenergetics, and chemostat culturing. BSI also recruited an entry level microbiologist.

\section{Program Development and Scientific Partnerships}

High quality proposals for multi-year grants, programprojects, and centers of excellence, totaling over $\$ 72 \mathrm{M}$, were submitted to DOE and NIH in response to calls focused on proteomics, computational biology, imaging, and microbial research. Notices of awards were received for new projects in such areas as:
- DOE-OBER, \$1.2M Low Dose project "Mechanisms of three-dimensional intercellular signaling," L. Opresko, P.I.

- DOE NABIR, Parallel Proteomic Identification of metal reductases, Mary Lipton, P.I., \$0.9M new funding in FY2004.

- NIH-NCRR, \$10.7M grant to establish a Proteomics Research Resource for Integrative Biology. The resource center will focus on the development and integration of novel analytical, instrumental, and biological strategies for studying expressed proteins, their complexes and post-translational modifications. The three technology development aims are 1) apply our single-chain antibody technology to improve proteome sample processing and isolation of protein complexes, 2) develop improved mass spectrometry-based approaches for proteomics, and 3) develop improved bioinformatics and software tools to analyze proteomics data. The broad, multidisciplinary nature of this project highlights a major strength of PNNL.

- NIH-General Medicine grant for studies of several trauma cytokine proteomics with Massachusetts General Hospital, \$1.2M, David Camp, P.I.

- NIH-Neurological Disorders grant to study molecular mechanisms of ischemia with Roger Skinner of Oregon Health Sciences University, \$0.6M, William Cannon, P.I.

\section{Technical Achievements}

As a major research PNNL thrust, systems biology is relatively new but has already made significant impacts on various DOE programs. Because of our special expertise in high-throughput proteomics and microbial systems, we are a key contributor to DOE's Genomesto-Life program. In environmental research, we are 
making strong contributions to the NABIR, EMSP, and Carbon Sequestration programs. In environmental cleanup, we have successfully provided comprehensive proteomics coverage of deinococcus radiodurans, the most radiation resistant organism known to date. Such whole proteome analysis is critical to understanding biological systems and how cells respond to external stresses such as radiation or chemical toxicity. In national security, proteomics is applicable to the characterization of biowarfare agents and antibodies for pathogen detection. In FY2002, we reported a breakthrough in singlechain antibody generation with the creation of a "library" with over $10^{9}$ distinct types of antibodies and demonstrated a high-throughput capability to select antibodies. In FY2003, we advanced this capability to create affinity-probes suitable for detecting pathogens and cancerassociated proteins at a speed far exceeding that of any previous technique. During 2003, we also successfully performed the most complete analysis of human plasma proteome, almost doubling the amount of biomarkers available for drug testing and for studying health effects, such as those related to low-dose radiation exposure. Based on these successes, we have recently contracted with the National Cancer Institute to help generate chips to identify cancer biomarkers.

Cytomegalovirus (CMV) Proteomics Project. To achieve a comprehensive proteome comparison of five CMV strain variants, whose genomes are currently being sequenced, it is important to obtain significant proteomic data (greater than $50 \%$ of predicted open reading frames) on all five strains (David Camp and Richard Smith, PIs). Using high resolution capillary chromatography combined with high sensitivity, high dynamic range FTICR MS measurements, and employing the accurate mass and time (AMT) tag approach with HCMV viral particles, the most complete viral proteome coverage to date has been achieved. Thirty two viral proteins, not previously detected in HCMV virion particles using any other experimental methods, were detected-a significant breakthrough for PNNL. Processing the AMT tag data on the viral proteins using new software tools being developed in our laboratory is providing quantitative information on the relative abundances of the viral proteins in the purified virion particles. The relative abundance data from FTICR MS measurements will be correlated with other quantitative experimental dataprimarily from quantitative Western blots of specific viral proteins, but possibly also with analytical SEM measurements of viral particles (i.e. quantitation of viral coat proteins). In addition, greater than 75 human host proteins, associated with the purified intact virion particles, were confidently identified by LC-MS/MS. A manuscript reporting these results is currently in preparation in collaboration with Jay Nelson, our OHSU collaborator.

Protein-Protein Complexes Project. This is a feasibility study demonstrating ability of $\mathrm{scFv}$ antibodies for affinity purification of proteins from organisms outside the scope of the current GTL (Robert Siegel, PI). The milestone-identification of the major constituents in at least one protein complex using mass spectrometry-is near completion this fiscal year. The goal of this project is to determine whether $\mathrm{scFv}$ antibodies can reliably isolate protein complexes from cellular lysates. In FY2003, $\mathrm{scFv}$ antibodies that specifically bind to calmodulin (a ubiquitous signaling protein responsible for intracellular calcium homeostasis) were identified and isolated from a screen of our pre-existing library. It was determined that these antibodies recognize different conformations of calmodulin. Purification strategies were developed for expressing soluble antibodies from multiple organisms that optimized both yield and ease of purification, prerequisites for applications such as immunoprecipitations. Assays were developed to quickly ascertain not only the activity of these purified antibodies, but also where they bound non-overlapping epitopes.

Preliminary work in developing a scaffold that could transform potentially unstable $\mathrm{scFv}$ into more stable and userfriendly antibody fragments was performed. Key issues that determine the success of any particular immunoprecipitation reaction include the ability of the affinity reagent to bind the antigen in the cellular lysate and the level of non-specific background that interfere with data interpretation. First attempts to immunoprecipitate calmodulin from mouse brain lysates were unsuccessful because of high levels of background for MS analysis and subsequent protein identification. A protein complex will be identified using an scFv antibody this Fall (November 1, 2003), but those antibodies may recognize antigens other than calmodulin as specified. Development will continue.

Single-Chain Antibody Project. This project addresses production of high affinity $\mathrm{scFv}$ antibodies (Michael Feldhaus, PI). Isolating the antigen specific 


\subsubsection{0 (cont'd) \\ (PEFA 1.5.1)}

$\mathrm{scFv}$ clones using a variety of selection techniques, including multiplex screens, individual screens, screens accomplished by flow cytometry, magnetic bead, based screens, or a combination of two techniques, was accomplished this year. The complete characterization for affinity, purification, and epitope binning was completed, which will now lead to the creation of a suite of plasmid vectors and yeast strains to generate a very diverse and highly functional antigen-binding fragment $(\mathrm{Fab})$ library to obtain affinity reagents for a myriad of uses.

\section{Strengthening Scientific Reputation}

More than 60 papers have been submitted for publication in peer-reviewed journals in systems biology. Emphasis was placed on papers that highlight research results in fields of study relevant to systems biology, especially those that were co-authored by multidisciplinary teams. Papers have been published in notable journals, including Journal of Proteome Research, Proteomics, Biochemistry, Cell Biology, Journal of the American Society of Mass Spectrometry, Proceedings of the National Academy of Science, and Biophysical Journal.

\section{Peer Review}

The BSI Advisory Review Committee met in August and provided invaluable guidance and councel, with specific comments regarding the initiative's strategy, scientific and technical direction, collaborations and partnership alliances, market segment targets and future facility strategies. A final report submitted to the DOE-PNSO and PNNL's Research Council, includes these key observations and recommendations:

- The BSI is positioned remarkably well; has made great technical progress; has a strong and visionary leader, impressive people in senior ranks and within the over- all technical team. There are fabulous facilities available that help the BSI do cutting-edge science. It can be considered "best in class" in cell-cell signaling biology and environmental microbiology.

- This is the best articulation of how the capability projects support each other and achieve objectives in the four thrust areas for eukaryotic and prokaryotic investigations and the relationship of a sound technology base. The committee recommended that the connections between strategic objectives and technical approaches be more clearly demonstrated.

- Excellent growth opportunities are recognized and the market analysis document is first rate. Continue to pursue large NIH programs and consider new markets within the DOD and DHS. The committee recommends that the BSI now develop a plan to capture these markets. To mitigate risk, diversification by identifying options for systems biology research outside the GTL program is an important safeguard. As a PNNL-transforming initiative, the BSI should increasingly emphasize impact on other PNNL areas.

- The committee indicated that key science drivers and technology underpinnings are strong; progress is outstanding, science \& technology are first rate, personnel development and collaborative interactions are excellent. The profile and stature of PNNL in the biology world is growing dramatically. The committee recommended that the connections of technical approaches to general systems biology, and particularly the GTL Facility II, be more explicitly identified to aid in time-frame prioritization. The BSI should consider an increasing role for microfabrication and microfluidics technologies. 


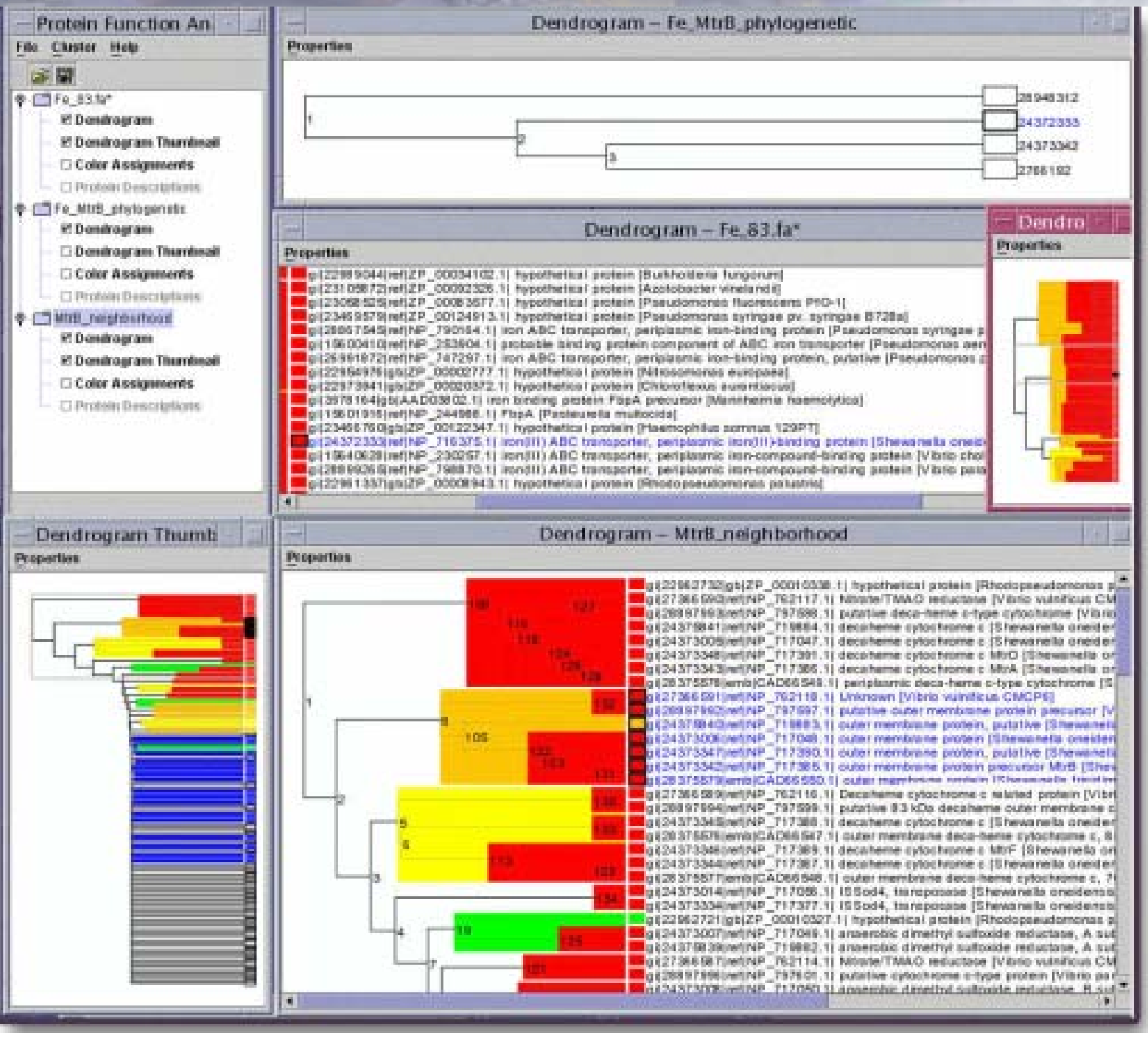

Figure 1.1.10. Similarity Box software provides integrated visualization and navigation of large-scale sequence data for data mining and genome comparison strategies. 


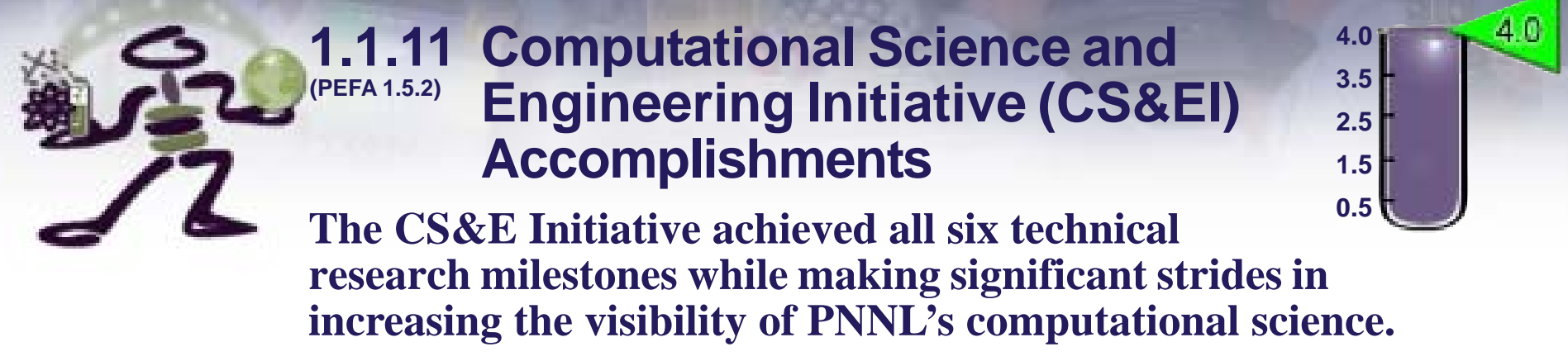

\section{Continued Technical and Scientific Progress}

The CS\&E Initiative continued to build computational capability across a broad spectrum of research areas. The six projects summarized below exemplify some of these advances.

- Modeling improvements in molecular science and nanoscience. The work extended the Self-Consistent-Charge Density Functional Tight-Binding model to include atomic forces and coupled them to drivers for energy minimization, transition state determination, force constant calculation, and molecular dynamics simulations. This enhancement permits quantum modeling and simulation of systems that are at the heart of biochemical reactivity and nanoscale science. This performance objective has been fully met.

- Remote sensing. The project team incorporated an open source version of Splus, called " $R$," in a parallel computer environment. This approach provided additional benefits for other statistical problems in addition to Bayesian regression. The team has produced a very successful parallel implementation of the Bayesian regression software, performed timing studies, and run a set of test cases to fully meet the defined goal.

- High-performance image analyses. The project completed development of the Parallel Computational Environment for Imaging Science (PiCEIS) workbench prototype using in-core and out-of-core processing of large image data sets (Figure 1.1.11). With the completion of the prototype, the project has successfully met the outcome goal.

- Computational engineering. The project team completed the development and implementation of a damage model for short-fiber composites using a multiscale mechanistic approach. The experimental work is also right on schedule and met the goal.
- Computer science. This project developed a novel visual data mining method to provide exploration and navigation capabilities to our ongoing whole-genome alignments work. The delivery of these methods fully met the objectives of this goal.

- Environmental science. A one-dimensional test problem involving the migration of carbon tetrachloride through Hanford soils was successfully completed using the parallel implementation of the Water-Air-Oil operational mode of the STOMP simulator on the Molecular Science Computing Facility Supercomputer. A three-dimensional test problem developed under the Hanford Science and Technology project by Mart Oostrom and Mark Rockhold was used for the final verification of the parallel implementation of the Water-Air-Oil operational mode of the STOMP simulator. The successful execution of these threedimensional simulations on multiprocessor computers completed the development and demonstration requirements for the parallel implementation of the WaterAir-Oil operational mode.

\section{Increasing External Visibility of PNNL's Computational Science Research Activities}

CS\&EI staff published, presented, conducted seminars, hosted visitors, and revamped its website to increase visibility of the computational capability at PNNL. Specifically:

- 12 peer-reviewed publications were submitted as a result of the work being performed in this initiative. Exceeded goal.

- A total of 9 presentations on computing, math/statistics were made at national or international conferences and DOE strategic workshops. Exceeded goal. 
- The CS\&EI Seminar Series was conducted between Feb 26 and May 28. The seminar series included 14 presentations by internal and external presenters. Exceeded goal.

- VIP visitors included Secretary Abraham and Director Orbach during the recent Super Computing 2002. Fully met goal.

- The PNNL Computational Science and Engineering web site was completely revamped. Fully met goal.

\section{Peer Review}

The CS\&EI Advisory Committee Review was conducted on Aug. 13-14, with five external and four internal reviewers. The review was highly successful, with the initiative receiving very positive comments for progress against initiative objectives.

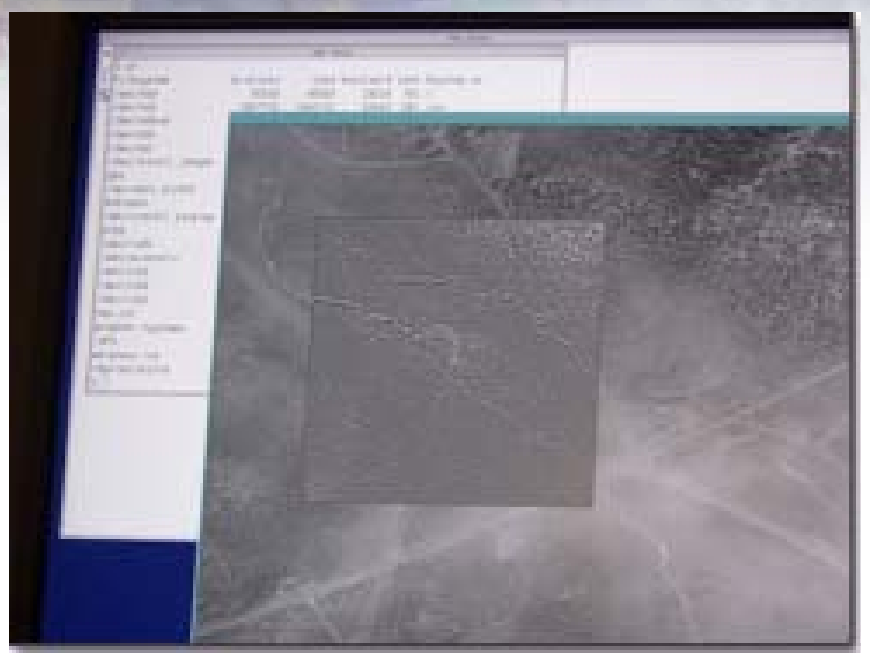

Figure 1.1.11. Working in close collaboration with the Imaging Sciences Initiative, CS\&EI researchers developed a parallel imaging toolkit that allowed processing of very large data sets supporting near real-time processing of satellite imagery. 


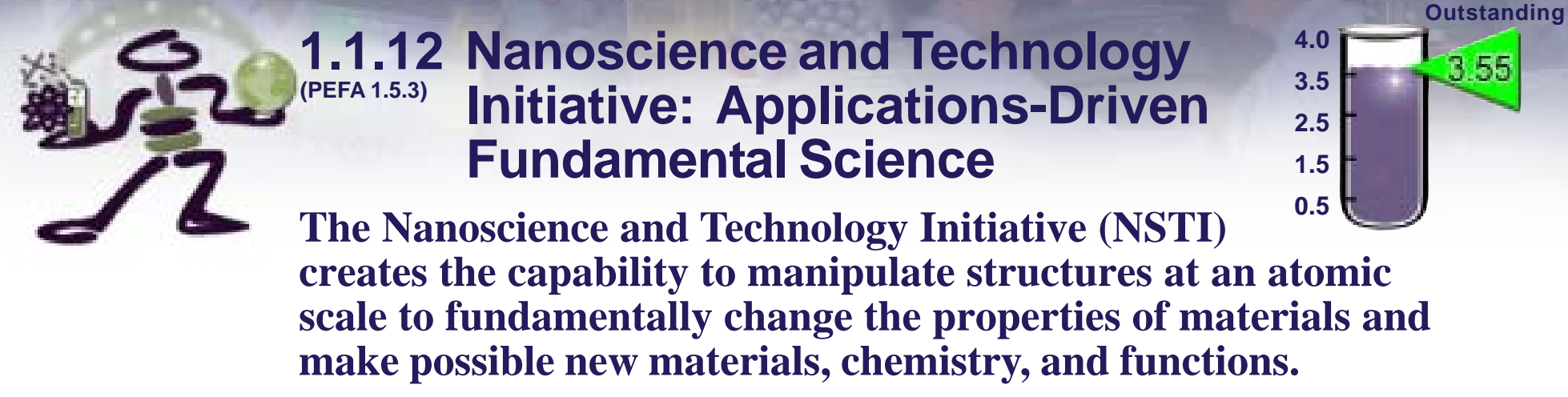

The NSTI continued to build capability at PNNL in our thrust areas of nanoscale catalysis, nanobiology, and advanced sorbent materials for preconcentration and detection. We also completed early-stage development work on a new system of dilute magnetic semiconductors based on the anatase system, and successfully transitioned separate aspects of the program to Office of Science and DARPA funding. This new semiconductor represents an enabling materials system for the emerging field of "spintronics," which has previously been limited by the lack of magnetic semiconductors with a Curie temperature much above that of liquid nitrogen. In contrast, thin films of Co-doped anatase have been shown to retain their magnetization at temperatures in excess of $300^{\circ} \mathrm{C}$. Using NSTI seed funding, we have developed oxygen plasma-assisted molecular beam epitaxial techniques for thin film growth of the doped anatase system and shown that Co dopant atoms substitutionally replace Ti atoms in the semiconductor-a subtle but important distinction from clusters of neutral Co atoms, which could also give a magnetic response.

\section{Scientific Impact}

Overall, NSTI achieved a high degree of success on three of its four stretch scientific goals set for FY2003, with significant progress and a high likelihood of success on the remaining one. In nanocatalysis, we quantified changes in the oxidation rate of $\mathrm{CO}$ on nanoscale metal clusters as compared to bulk metals and demonstrated the increased thermal stability of small Pt metal catalyst particles over bulk or micrometer scale particles. In nanobiology, we demonstrated silicate-armored enzymes with orders of magnitude increased stability over unarmored (or free) enzymes in solution and created immobilized arrays on conductive nanoporous matrices. The silicate coating is assembled via a crosslinked polymer built on chemically modified surface groups of the enzyme and results in a "single enzyme nanoparticle" or SEN, which can be rendered soluble or alternatively immobilized in a mesoporous silica matrix or attached to a conductive nanowire array. For example, we have demonstrated attachment to arrays of oriented multiwalled carbon nanotubes. We are also perfecting the synthesis of arrays of conductive polypyrrole nanowires, grown by a controlled electrochemical technique from a monomer solution for future immobilization studies. The high effective area conductive substrate is required to enable signal transduction from the enzyme without limiting mass flow in biodetection applications. Most importantly, we showed that the armor coating on the enzyme does not significantly reduce the mass transfer rate of reaction with a substrate. We continue to investigate how the structure of the shell affects the reaction kinetics of the enzyme and to probe the limits of SEN lifetime in various environments. As we build this understanding, we hope to enable nanobiological machines based on enzymes which have been stabilized outside of the cellular environment. Once the scientific questions are answered, we envisage futuristic applications such as a smart vest made from fibers loaded with SENs, which can both warn and protect the wearer from biohazards in real time.

\section{Increased Visibility}

NSTI met or exceeded all the visibility-enhancing goals related to in publications, presentations, seminars, and overhauling the website. In addition to funding research to build a strong nanoscience capability at the laboratory, NSTI continues to act as a central point of contact, coordinating and increasing the visibility of nanoscale research at PNNL. We have continued to support the regional Joint Institute for Nanoscience (http:// www.nano.washington.edu/pnnl/jin.asp) and we are expanding our regional activities into the Northwest Nanoscience and Nanotechnology Network $\left(\mathrm{N}^{4}\right)$ with 
the launch of a new coordinating website www.pnl.gov/ nwnano. Strengthened regional collaborations have led to the development of intensive two week short courses in aspects of nanoscience taught by PNNL staff and faculty at the University of Washington, Washington State University and the University of Idaho. The first two courses occurred in 2003 and covered Nanoclusters, Nanomaterials and Nanotechnology and Theory of Nanoscale Systems, respectively. Staff also accepted invitations to present at the DOE National Science Bowl and provided content for local magazines, colleges and public broadcasting stations.

\section{Project and Program Development}

In program management, NSTI-funded research was critical to at least four separate proposals to DOE Office of Science. Significant new efforts in catalysis science and bioremediation were funded. To help direct the Initiative, our distinguished Advisory Board was reconvened with five out of the original six external members attending and the remaining participant contributing by email. The Board concluded that "NSTI is positioning the Lab in important mission areas" and should be extended as a longer term initiative targeted at providing needed fundamental science to enable new mission-oriented applications. NSTI also hired a new staff member with expertise in spintronics and is successfully building nanobiology capability internally instead of recruiting externally.

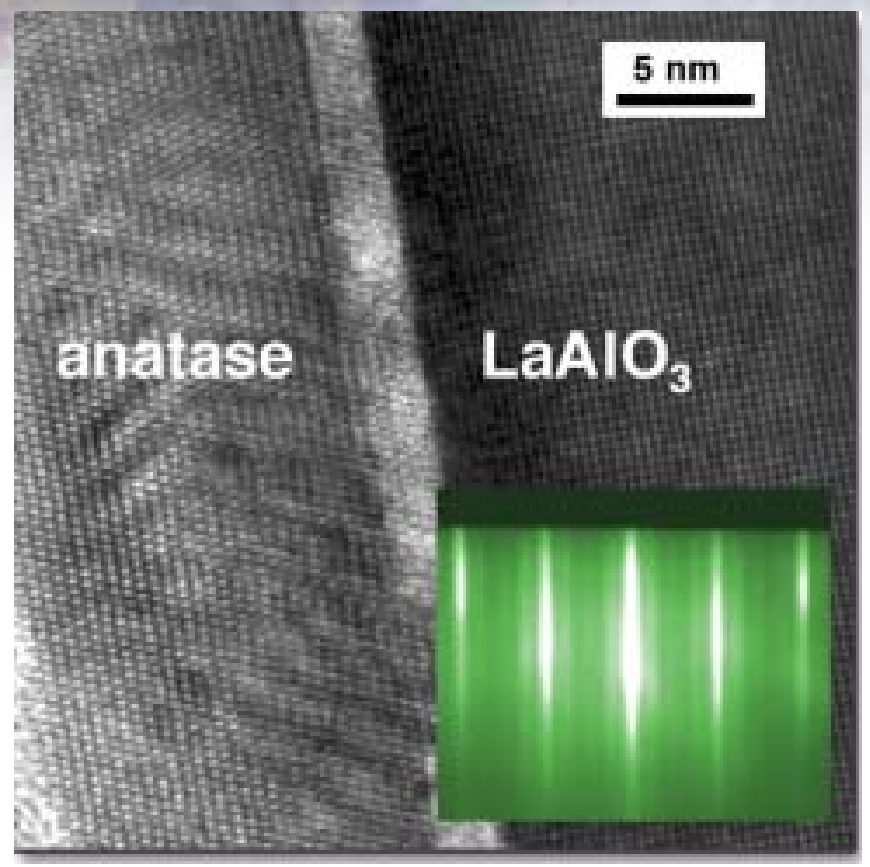

Figure 1.1.12. The development of a new magnetic semiconducting system: Bright field TEM lattice image showing individual atoms of a $16 \mathrm{~nm}$ thick $\mathrm{TiO}_{2}$ film grown epitaxially on $\mathrm{LaAlO}_{3}(001)$ shows the high quality crystalinity of the semiconducting layer. Micrograph taken using the EMSL microscopy facility. (Inset: RHEED pattern along [100] axis of a $21 \mathrm{~nm}$ thick film doped with $6 \% \mathrm{Co}$, showing an extremely flat, layer by layer growth mode). 


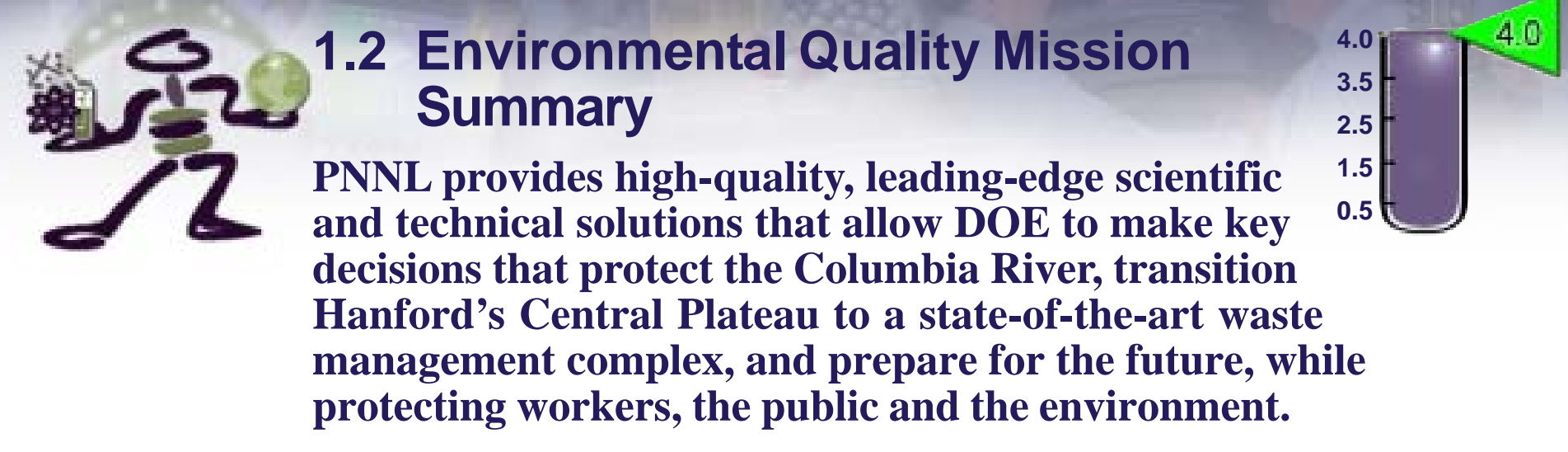

PNNL provides DOE with the science and technology basis to make sound decisions necessary to protect and remediate Hanford Site groundwater and the Columbia River. The Groundwater Protection Project's mission is contaminant source control and groundwater remediation to protect the Columbia River. PNNL manages three projects that are integrated with Hanford's Groundwater Protection Program: Remediation and Closure Science, Hanford Site-wide Assessment, and Groundwater Performance Assessment.

The modules in this section present examples of quality, relevance, and research program management from the perspective of PNNL's environmental quality mission. Modules under this mission are summarized below into the categories of objectives 1.1, 1.2, and 1.4: Quality, Relevance, and Research Program Management.

1.2.1 Peer Review. In FY2003, the ETD peer review process evaluated not the quality of past accomplishments but the future direction of PNNL's environmental science and technology development. The April 2003 meeting the Directorate Review Committee was charged to "understand and validate the new ETD vision and strategy." Overall, the DRC was "... .very pleased with the progress ETD has made over the past year and [expressed] hope that the review and comments would make ETD even more successful in the year to come."

1.2.2 Pnnl's R\&D And Assessment Projects and Their Relevance to Hanford's Groundwater ProtecTION Mission. PNNL provides DOE with the science and technology basis to make sound decisions necessary to protect and remediate Hanford Site groundwater and the Columbia River. The Groundwater Protection Project's mission is contaminant source control and groundwater remediation to protect the Columbia River. PNNL manages three projects that are integrated with Hanford's Groundwater Protection Program: Remediation and Closure Science, Hanford Sitewide Assessment, and Groundwater Performance Assessment.

The Remediation and Closure Science Project (RCSP) continued to investigate contaminant fate and transport at tank farms and waste sites. Initial data from Hanford Site-wide Assessment Project are being used to make remediation decisions, including an assessment of Hanford's composite impact for inclusion in the Solid Waste EIS. PNNL's Hanford Site-wide Groundwater Model provided the basis for fate and transport scenarios used to support the Hanford Solid Waste EIS, the preliminary risk assessment of the Integrated Disposal Facility in 200 East Area, and the Tank Closure EIS.

1.2.3 Hanford Site Work on Tank Waste, Safe Storage and Retrieval, and the Development of Supplemental Treatment Technologies. During FY2003, we provided significant science and technology contributions to ensure the safe storage and retrieval of Hanford's radioactive tank wastes. We also provided senior management and technical support to enable DOE and CH2M HILL to evaluate treatment options for transuranic (TRU) sludges and for supplemental processing of up to $70 \%$ of Hanford's low activity tank wastes (LAW).

1.2.4 PnNL's Work in Support of Hanford's Central Plateau Restoration Activities. In support of Fluor Hanford (FH) and DOE, PNNL provided science and technology solutions to help resolve critical spent nuclear fuel issues and enable the stabilization and safe storage of Hanford's plutonium. We developed the scientific basis for 
modifying the plutonium stabilization and packaging standards. The large inventory of items accumulated from various DOE sites could not be stabilized at high temperatures without causing extensive corrosive damage to the processing equipment. PNNL scientists, with others, showed that stabilizing these plutonium compounds at a lower temperature would be effective and safe. This change was implemented, obviating the need to develop, design, and install equipment that could withstand corrosion at high temperatures.

1.2.5 Pnnl's Program Management Performance in Support of Rl and the Office of River ProtecTION. Having provided radiation protection services to the Hanford Site for more than 40 years, PNNL has developed the technical expertise and understanding of customer needs, the Hanford radiological environment, and regulatory drivers to provide radiation protection services of the highest quality for DOE-RL and Hanford contractors. This support continued in FY2003. We also completed the review, update and publication - on a highly accelerated schedule - of the revised and much expanded draft and final Hanford Solid Waste Environmental Impact Statement (HSW EIS). During the process, we gathered and addressed more than 5,000 public comments. Finally, PNNL provided DOE-RL with the technical basis, analysis, and planning for accelerated, quality Hanford cleanup, including products that clarify and communicate this vision.

1.2.6 PnNL's Work for Doe-Rw and Doe-Eh. PNNL has also provided significant science and technology contributions to DOE-RW in support of the nation's High Level Waste Repository, and to DOE-EH for protection of the workers, the public and the environment. Through RPL, we contributed to the Yucca Mountain license application baseline for waste form performance; as a result of this work, alternative hypotheses have been put forward that provide thought leadership on future waste form testing.

We also made significant contributions to DOE$\mathrm{EH}$ for protection of the workers, the public and the environment. We continued to provide technical support to DOE Laboratory Accreditation Program by managing the Phantom Library used to calibrate whole body counters throughout the country, and by upgrading the bioassay program by developing an internal dosimetry intercomparison program. Finally, we provided significant technical support to DOE-EH's Mayak Dose Reconstruction Project.

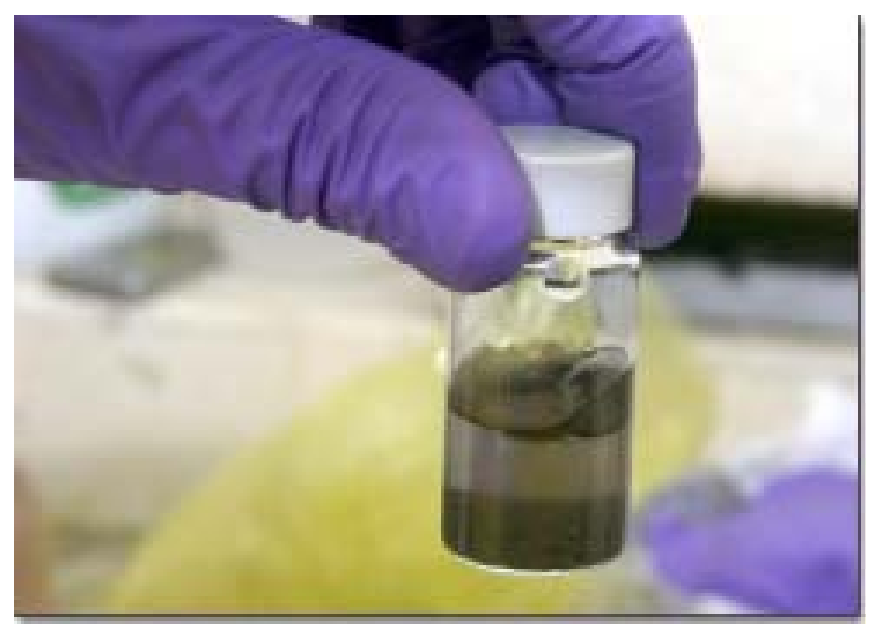

Figure 1.2. PNNL has proposed to study the molecular mechanisms of new observations of hydrophobicity in uranium, to DOE-RW. Hydrophobicity (the condition of not binding to, or dissolving in, water - as seen in the photo) might be a key reason that uranium deposits are stable for millions of years in nature. The results of this work could greatly improve current understanding of waste form durability in the Yucca Mountain waste repository. 


\section{In FY2003, the Environmental Technology Directorate's (ETD) Directorate Review Committee evaluated the future direction of PNNL's environmental science and technology development.}

The 2003 Directorate Review Committee (DRC) meeting for the PNNL's Environmental Technology Directorate (ETD) was held in Richland, Washington, April 1 and 2, 2003. All eight members of the DRC were present, including Chairman Dr. John T. Whetten. In addition, Dr. Lawrence R. Curtis, Professor and Head of the Department of Environmental and Molecular Toxicology at Oregon State University, attended as a guest Committee member.

Before the April 2003 meeting, the Directorate Review Committee was charged to "understand and validate the new ETD vision and strategy." As a result, the DRC's review was not a review of the Directorate's research or accomplishments; it was, instead, a forward-looking critique of the Directorate's plan for the future, as best the committee could understand it during the two-day meeting.

\section{Strategic Planning Process}

The Committee noted that the "strategic planning process conducted by ETD in 2003 differs significantly from the process followed in previous years. Recent strategic plans for the Directorate have been developed around customers and product lines. This year, however, the plan was built around the capabilities of the Directorate's four Divisions, which resulted in a process that greatly empowered the Division Directors. Morale has notably improved. The resulting plan was a broad, ambitious plan, which defines a number of key initiatives in each Division...."

While the DRC generally applauds the output of ETD's strategic planning process, it recognized that it was presented with the results of an effort that was only recently completed. The Committee commented that ". . . there is much more to be done before the process can be considered complete. For example, the linkages between the Laboratory's mission, ETD's mission, and the Capability Development Focus Areas (CDFAs), need to be clearer and more explicit."

\section{Capability Development Focus Areas}

The DRC gave consideration to ETD's recently developed Capability Development Focus Areas (CDFAs)areas of technical capability each Division intends to cultivate to effectively address future customers' needs and assure long-term visibility. The DRC provided comments on each of the key CDFAs.

In addition to reviewing ETD's Strategic Planning Process and the development of CDFAs, the Committee also commented on such topics as the Changes in EM Business; 300 Area Transition and Facility Planning; New Opportunities, including the transition of the Marine Sciences Laboratory and within the arena of Homeland Security; and ETD's Regional University Relations with Oregon State University and Washington State University.

\section{Observations and Recommendations}

In addition to specific comments on the ETD strategic planning process, the CDFAs, and the topical areas provided above, the Committee made several general observations/recommendations.

First, DRC meetings are very good and continue to get better. Complex material is presented concisely, and the discussions are frank and open.

Second, the DRC commends the ETD leadership development process. The committee commented that "we have no idea what the process is (we believe it would behoove the Laboratory to find out), but we know that the results have been spectacularly successful. It appears the Directorate is constantly graduating its 
leadership to other parts of the Laboratory, and then replacing those leaders with new ones from lower on the leadership development ladder. Indeed, in a relatively few years most of the ETD leadership has permanently or temporarily moved to other jobs, and the Directorate doesn't seem to have missed a beat."

Third, the Committee is concerned that it may be increasingly difficult for PNNL to maintain and strengthen its R\&D infrastructure in the face of declining EM support. The 300 Area transition is a prime example.

Finally, two committee members noted during a visit to the Marine Sciences Laboratory (MSL), that "MSL scientists had done some very good work, but generally were not well known in the broader scientific community. The committee recommended publishing the results of their research in publications like Scientific American or American Scientist that would be read by a broad segment of the scientific community. In fact, the committee felt this suggestion may have merit for the entire Directorate. The charter of the Directorate is very broad, and Directorate scientists and engineers have a good record in $R \& D$ - ranging from environmental protection and clean-up, to studies of fish and other aquatic organisms. ETD should consider targeting topics of special interest for general articles for the broad scientific community."

\section{Summary}

Overall, the DRC was pleased with the progress ETD has made over the past year and expressed hope that the review and comments would make ETD even more successful in the year to come.
The DRC was particularly complimentary of the past leadership of Dr. Walt Apley. Dr. Apley's recent move from ETD Associate Laboratory Director to Interim Laboratory Director; and Dr. Rod Quinn's subsequent appointment from Division Director to Interim ETD Associate Laboratory Director were hailed as a model transition. "Each had the experience and know-how to move into the new jobs, and, since the two have great respect for each other, they were able to make, what appears to us, a 'flawless' transition."

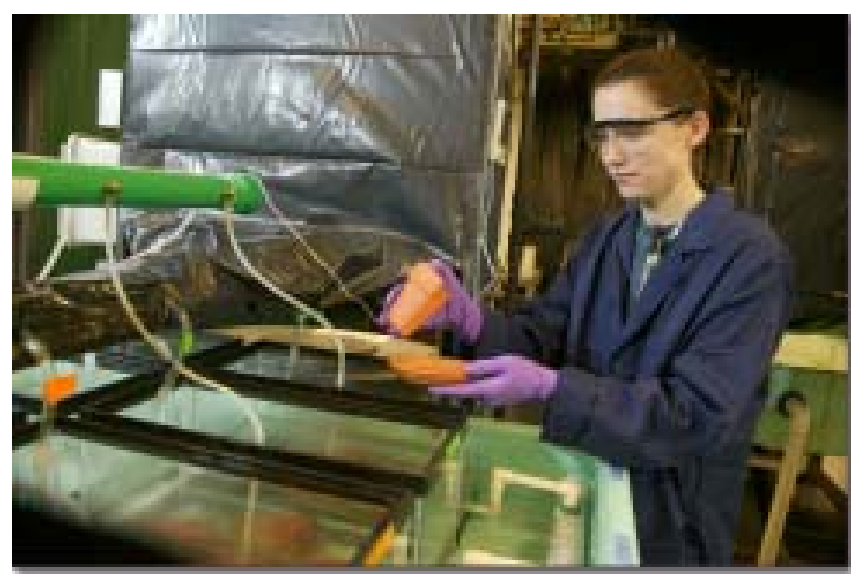

Figure 1.2.1. Researchers at the Marine Sciences Laboratory are developing a suite of tests and screens for identifying chemicals that alter or impair the endocrine systems of humans and wildlife. Researchers use breeding "barns" to collect eggs from fathead minnows as a part of these experiments. 
1.2.2 PNNL's Research and Development (R\&D) and Assessment Projects and their Relevance to Hanford's Groundwater Protection Mission

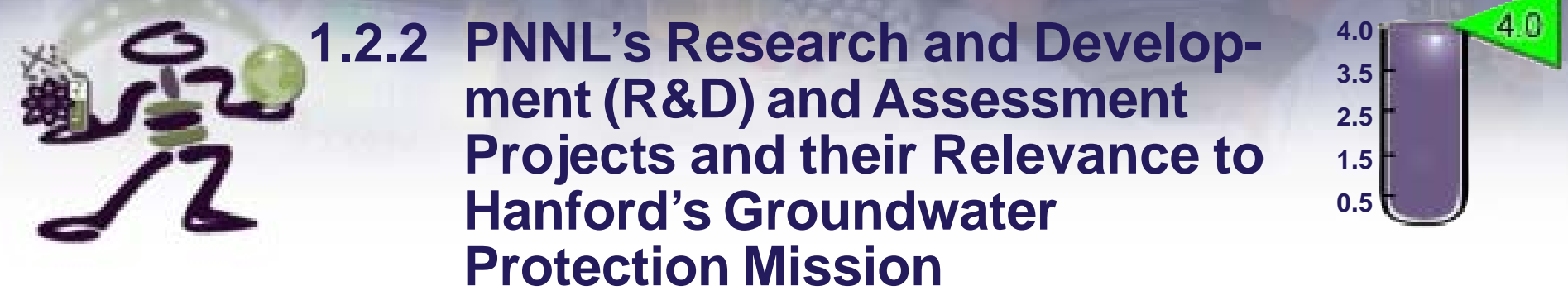

\section{PNNL's science and technology gives DOE Richland Operations the scientific and technical information and tools to make sound decisions about protecting and remediating Hanford Site groundwater and the Columbia River.}

PNNL manages three projects integrated with Hanford's Groundwater Protection Program: Remediation and Closure Science (RCSP), Hanford Site-wide Assessment, and Groundwater Performance Assessment. RSCP efforts support characterization and remediation activities by both the Central Plateau (Fluor) and the Tank Farm (CHG) contractors creates the basis for remediation decisions and actions.

\section{FY2003 Remediation and Closure Science Accomplishments}

- Soil Inventory Model of the System Assessment Capability for 311 past practice soil waste disposal sites to provide data for the FY 2004 Composite Analysis.

- Appendix D for the B-BX-BY Field Investigation Report (FIR), containing RCSP and Environmental Management Sciences Program (EMSP) contributions to tank farm characterization. The research addressed important issues associated with transport of tank wastes and features of the vadose zone that control waste migration. The results explain attenuation mechanisms of strontium-90 and uranium in the subsurface and support geochemical contaminant behavior models, which are being extended to evaluate remediation alternatives at the $100-\mathrm{N}$ Area and 300 Area.

- Laboratory investigation of the physical and chemical association of uranium in 300 Area sediments collected from the vadose zone and aquifer. Detailed investigations of uranium geochemistry will help develop the rigorous conceptual model of long-term uranium behavior needed to support the 300-Area groundwater ROD.

- Laboratory results that materially refine our understanding of DNAPL behavior at Hanford, showing that
DNAPLs, such as carbon tetrachloride, form significant residuals as they move through vadose zone sediments. This effect, incorporated into simulations for Fluor, has not been included in previous models.

- Field experiments for reactive transport with nonradioactive strontium at a horizontal clastic dike near the ground surface that is representative of deep vadose zone layering at Hanford. These subsurface features cause lateral spreading of water and contaminants. Results have been used to evaluate migration of mobile contaminants from disposal of wastes to past practice waste sites and leaks from singleshell high-level waste tanks.

- Studies of strontium-90 uptake by periphyton (aquatic microflora); we also initiated studies of strontium-90 uptake by fish. These results, being incorporated into ecological risk assessment modules in the System Assessment Capability, will be included in other Hanford Site risk assessment models.

PNNL also provides programmatic leadership in science and technology by integrating research supproting the DOE Office of Science and the DOE-EM Office of Science and Technology. The following FY2003 activities supported this integration:

- Published Revision 2 of Science and Technology Roadmap, the basis for future work by the RCSP to support accelerated cleanup of the Hanford Site. This roadmap is linked with the Project Master Schedules, the long-range plans developed by Fluor.

- RCSP supplemented funding for well drilling at the 100-H Area to support a project within the DOE Office of Science Natural and Accelerated Bioremediation Research (NABIR) Program focused on bioremediation of chromium, which could be used for portions of the 100 Area chromium plumes. 
- RCSP interfaced with NETL contractor teams to characterize carbon tetrachloride DNAPL in the 200 West Area. The project provided data and information to support development of conceptual models for postulated DNAPL vadose zone migration.

\section{Hanford Site-wide Assessment Projects: Data Being Used to Make Remediation Decisions}

- An assessment of Hanford's composite impact was completed for inclusion in the Solid Waste EIS. Comments on the draft EIS requested that the impacts from solid waste burial grounds be put in the context of the contaminant contributions of the surrounding waste sites. The System Assessment Capability was used to estimate the impact of key contaminants from all waste sites to provide a regional context for future burial ground impacts in the Solid Waste EIS.

- The initial site-wide assessment was used to complete an assessment to quantify risk resulting from waste sites in each region of the Central Plateau. This information allowed quantitative ranking of the regions based on risk as one of the metrics for prioritizing sites for cleanup. The information was included in the Fluor Hanford document WMP-18061, Optimization Strategy for Central Plateau Closure.

Refinements made the site-wide assessment code more useful to DNFSB-required composite analyses, to begin in FY2004: we added the ability to simulate contaminant transport through the air and deposition on soil for uptake by plants, animals, and humans. Other modifications improved the representation of groundwater and river transport of contaminants. The inventory database has also been improved by incorporating the results of science and technology activities to better define waste site inventories.

The assessment team also met with Washington State Department of Ecology, the EPA, and the technical staff of the interested Tribes to discuss the results of an initial assessment of Hanford impact. These meetings allowed regulators and others to discuss changes planned in preparation for upcoming analyses.

\section{Groundwater Performance Assessment}

PNNL's Hanford Site-wide Groundwater Model provided fate and transport scenarios for evaluating Hanford Solid Waste disposal alternatives and cumulative impacts supporting the Solid Waste Environmental Impact Statement (SW-EIS), the preliminary risk assessment of the Integrated Disposal Facility in 200 East Area, and the Tank Closure EIS initiated in FY2003. The site-wide model and linked local-scale submodels will also provide fate and transport scenario analyses for key FY2004 Hanford assessments.

In addition, an innovative transient inverse calibration approach continues to be used to calibrate the alternative conceptual models of the unconfined aquifer system at Hanford to all available hydraulic head data. Efforts this past year have also been initiated to calibrate these alternative models to historical observations of tritium plume migration. The initial alternate conceptual models, and others yet to be developed, will allow analysts to quantify the full range of uncertainty in future predictions of groundwater flow and contaminant transport.

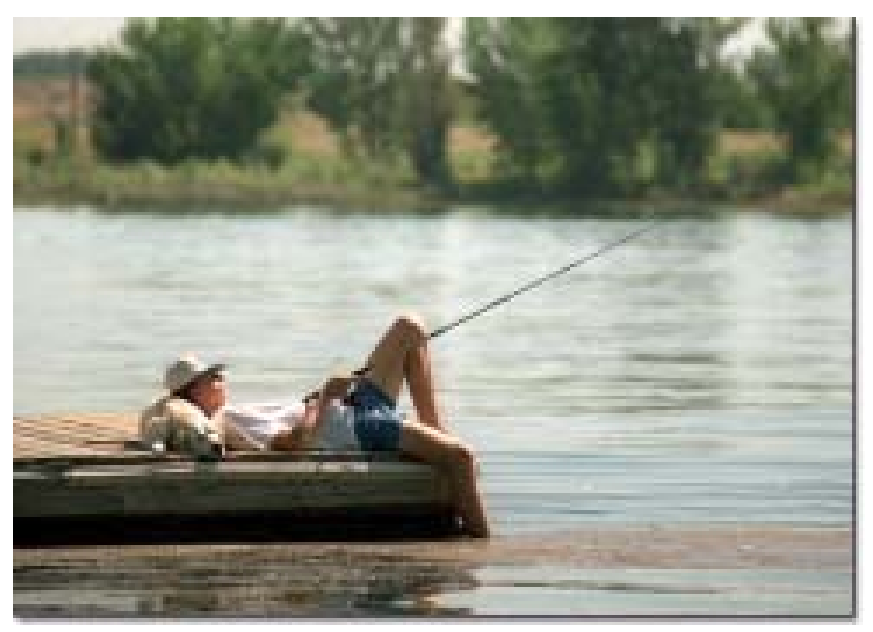

Figure 1.2.2. PNNL's science and technology is addressing key technical questions associated with the movement and cleanup of contaminated groundwater, and the protection of the Columbia River. 


\section{In FY2003, PNNL's science and technology contributions provided critical support to Hanford's tank waste, safe storage and retrieval, and supplemental treatment activities.}

Hanford has the largest volume of high-level radioactive tank waste in the United States, with the highest lifecycle cost for cleanup of any other program area and site within DOE's Environmental Management program. PNNL is providing key technical and programmatic assistance in support of tank waste safe storage, retrieval, supplemental treatment, and tank closure.

\section{Tank Waste}

During FY2003, we provided senior management and technical support to enable DOE and CH2M HILL to evaluate treatment options for transuranic (TRU) sludges and for supplemental processing of up to $70 \%$ of Hanford's low activity tank wastes (LAW). Key support in this area included:

- Characterization of physical and dewatering properties of TRU sludge materials and preparation of physical simulants to support testing and evaluation of TRU sludge transfer and dewatering operations;

- Development of a cold saltcake simulant recipe; and preparation, characterization, and delivery of equivalent batches of simulant to the supplemental treatment vendors to ensure a balance evaluation of each LAW treatment option;

- Preparation and delivery of pre-treated radioactive dissolved saltcake waste to vendors for use in validating waste simulant testing;

- Development of a balanced strategy to evaluate the relative performance of the waste forms from the three different supplemental treatment options;

- Completion of waste form characterization studies for two of the three options and completion of waste form performance calculations to support the risk assessment for a supplemental treatment selection decision process; and
- Hazard classification, hazard analysis, and nuclear safety \& licensing strategy development for supplemental treatment technologies

\section{Safe Storage and Retrieval}

PNNL also provided significant science and technology contributions to ensure the safe storage and retrieval of Hanford's radioactive tank wastes. Key contributions this year included:

- Development and delivery of the Remotely Operated Nondestructive Evaluation (RONDE) system for tank integrity inspections of the knuckle region of Hanford's Double-Shell Tanks (DSTs);

- Thermal and operating loads structural modeling and analysis of Hanford tanks to assess tank integrity issues;

- Critical technical support to accelerated retrieval for the areas of vapor release and aerosol generation predictions and testing;

- Scientific/engineering support with flammable gas issues for the Documented Safety Analysis in the form of technical document publication and reviews; and

- Assessment of several SST and DST waste mixing and mobilization operations and subsequent cross-site waste transfers to support accelerated waste retrieval and waste transfers to the Waste Treatment Plant.

\section{Supplemental Treatment Activities}

We provided extensive technical assistance to one of the supplemental treatment vendors in waste formulation development; and in lab, engineering, and field-scale testing of bulk vitrification technology. Key activities here included

- Development of a successful baseline glass formulation with radioactive confirmation testing; 
- Support of radioactive engineering-scale tests within the Radiochemical Processing Laboratory for process evaluation and support of a technetium material balance; and

- Waste form product durability evaluations for lab, engineering, and full-scale tests.

\section{Tank Closure}

We provided key analysis and testing of tank waste to support accelerated tank closure preparations, including completion of initial water and acid leach testing of waste from tank AY-102. Results showed that technetium-99 (Tc) is not completely water leachable as was previously assumed. The results were compared with previous water leaches of waste from tank BX-101 where all of the Tc was water leachable. It was concluded that future releases from the tanks as a result of leaching by water will be very tank specific. The results for AY-102 were documented in PNNL report Lindberg, M.J. and W.J. Deutsch. 2003. "Tank 241-AY-102 Data Report” PNNL14344.

We also provided key support to CH2M HILL by preparing data packages for Hanford's Tank Closure Environmental Impact Statement. PNNL provided leadership and coordination of several data packages, including retrieval and safety; provided technical content for the Closure data package, including details on the "clean closure" alternative and the tank farm barrier placement and monitoring; and co-developed information on gaseous and particulate emissions for five data packages associated with construction, operation, and D\&D activities.

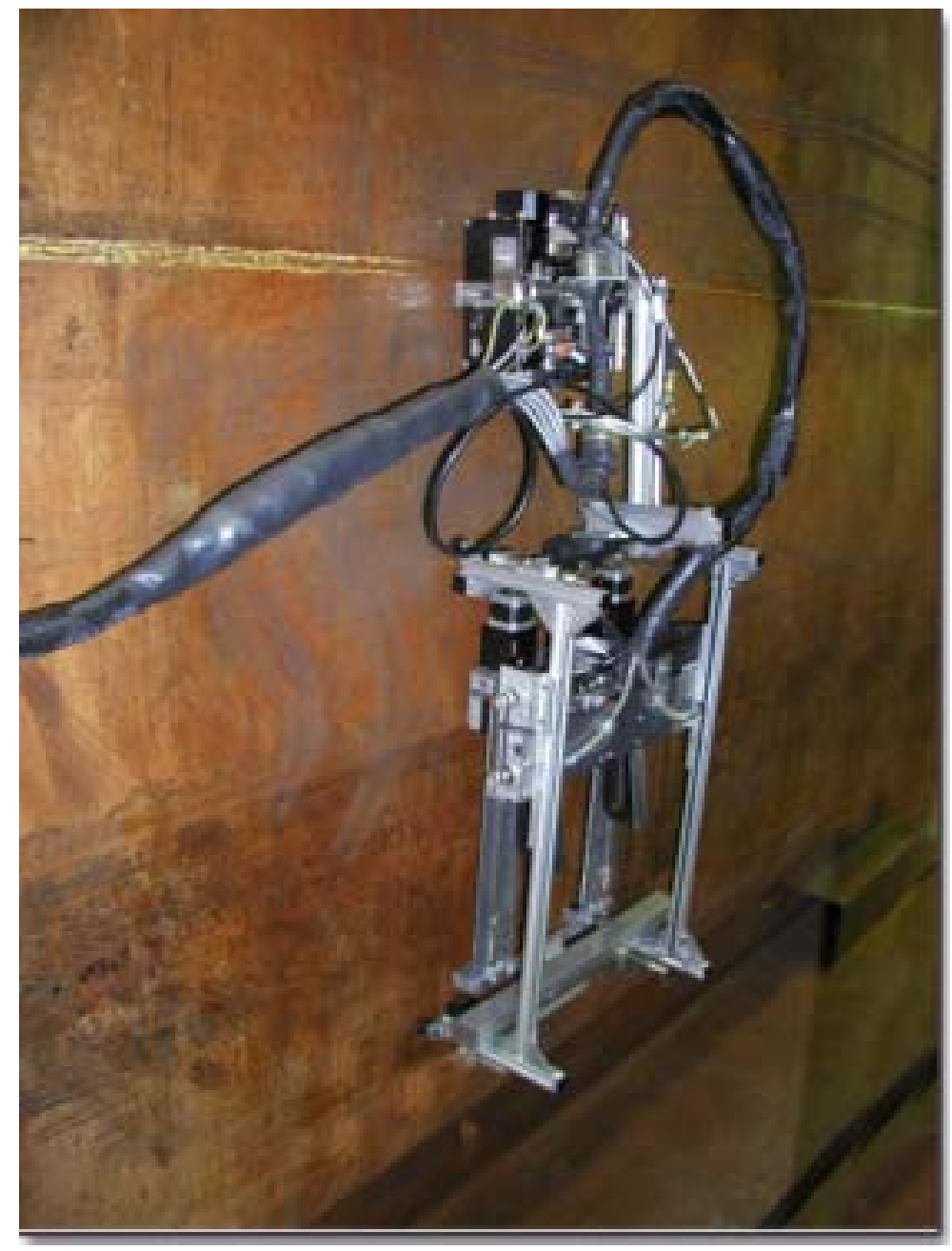

Figure 1.2.3. PNNL-developed robotic technologies provide important data on Hanford's waste tank integrity. The Remotely Operated Nondestructive Examination (RONDE) prototype (above) inspects the wall of a Hanford double-shell tank. 


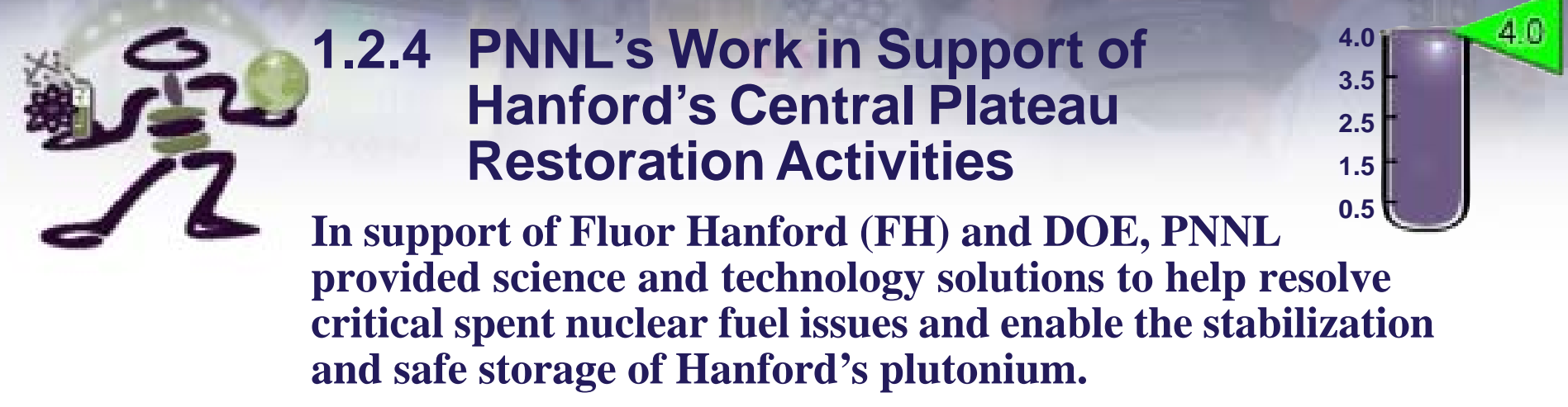

\section{Moving Forward on Spent Fuel Issues}

PNNL performed work critical for dry storage of spent nuclear fuel sludge, including laboratory and bench-scale studies to establish the technical foundation for design calculations and to establish a defensible safety basis for the retrieval, shipment, and storage of K-basin sludge. PNNL staff contributed to the development of alternatives for retrieval and storage, which were used in framing a request for proposals, and helped develop a Reasonably Bounding Case for handling the sludge. Careful analyses revealed unwarranted conservatisms that could be removed to provide for a more flexible design and broader operating conditions.

In support of basin closure activities, PNNL developed one-of-a-kind technologies to perform Non-destructive Analyses (NDA) of highly contaminated fuel storage basins. After the spent fuel, sludge, and debris have been removed from the K-Basins, efforts will turn to decommissioning the basins themselves. Over the past 20 years, the K-East basin has become heavily contaminated with highly radioactive materials that dissolved into the water from corroding spent fuel. PNNL designed a unique system of instruments that were deployed underwater in the basin to determine how extensively the concrete had been contaminated. The results from this effort revealed much higher contamination levels than anticipated, providing vital information to effectively plan for removal and disposal of basin materials.

We provided risk and decision tools to enhance project baseline planning and end-state decisions. PNNL staff developed new decision tools that incorporate uncertainty and historical project performance into Fluor Hanford's fee projections to provide a more realistic and complete basis for management decision-making. Specific assessments were performed for K-Basin sludge removal and deactivation projects, and a fee risk assessment was developed for dry storage of the cesium and strontium capsules. New tools were developed that integrate cost and schedule risk so that changes to the baseline can be quickly and comprehensively assessed.

\section{Plutonium Stabilization and Storage}

PNNL developed the scientific basis for modifying the plutonium stabilization and packaging standards. Prescribed procedures for stabilizing plutonium at high temperatures have been used for many years to assure various forms of the metal would be safe during extended storage. As a part of final cleanup efforts, a large inventory of items had accumulated from various DOE sites that could not be stabilized at these high temperatures without causing extensive corrosive damage to the processing equipment. PNNL scientists, in collaboration with others, were able to demonstrate that stabilizing these plutonium compounds at a lower temperature would be effective and safe. Implementing this change obviated the need to develop, design, and install equipment that could withstand corrosion at high temperatures.

More than 900 items of plutonium oxide containing variable concentrations of chloride salts must be stabilized and packaged for safe storage. We developed and optimized the processes needed to stabilize some of these most challenging plutonium legacy materials. After studying several options to remove the salts, we recommended using idle equipment remaining from earlier solutions processing in a slightly altered configuration. Subsequent prototype testing confirmed that this approach would be effective and practical. Fluor adopted the recommendations, and has been successfully processing these materials. This approach allowed the stabilization project to avoid the significant cost, time, and dose that would have been involved in cleaning out a glovebox and installing new equipment. 
PNNL also developed and qualified a non-destructive assay system to support requirements for accounting of plutonium. As the decommissioning of the Plutonium Finishing Plant accelerates in FY2004, waste will be packaged into drums and large boxes that must be carefully measured for small quantities of plutonium residues. The plant is equipped with instruments to measure the plutonium content in drums, but the expected commercial equipment to measure boxes was delayed by more than a year, forcing the need for a near-term solution. PNNL scientists developed a portable system to make the box measurements using existing in-house instruments and specialized software that provided the accuracy and precision required by the Safeguards program. This capability will allow decommissioning efforts to proceed with the loading of waste into large boxes, avoiding significant size reduction work that would be required to load the waste into 55 gallon drums.

Finally, we developed an alternative approach for decommissioning the Plutonium Finishing Plant. Current cleanout and demolition plans rely on extensive use of manual labor to carefully remove contaminated equipment and decontaminate the facility. An alternative developed by PNNL shows that large sections of the facility could be stabilized with grout, then cut into pieces that would be disposed in appropriate repositories. This option offers the opportunity to greatly reduce worker hazards and project cost, and will be incorporated into the regulatory decision process.

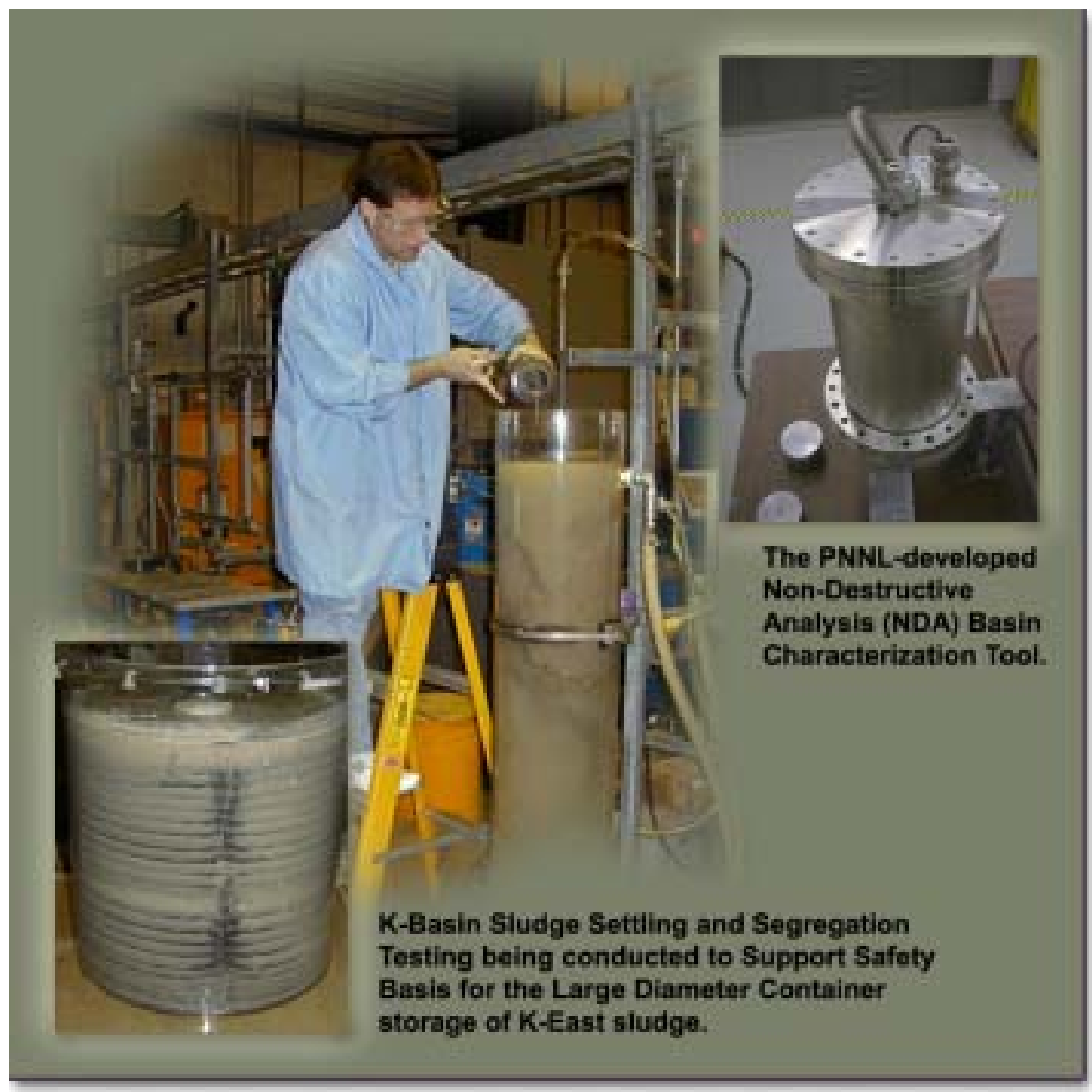

Figure 1.2.4. PNNL is conducting laboratory and bench-scale studies that help establish defensible safety parameters for processing and dry storage of spent nuclear fuel sludge, and developing one-of-a-kind technologies to perform NonDestructive Analysis (NDA) of highly contaminated fuel storage basins in support of basin closure activities. 


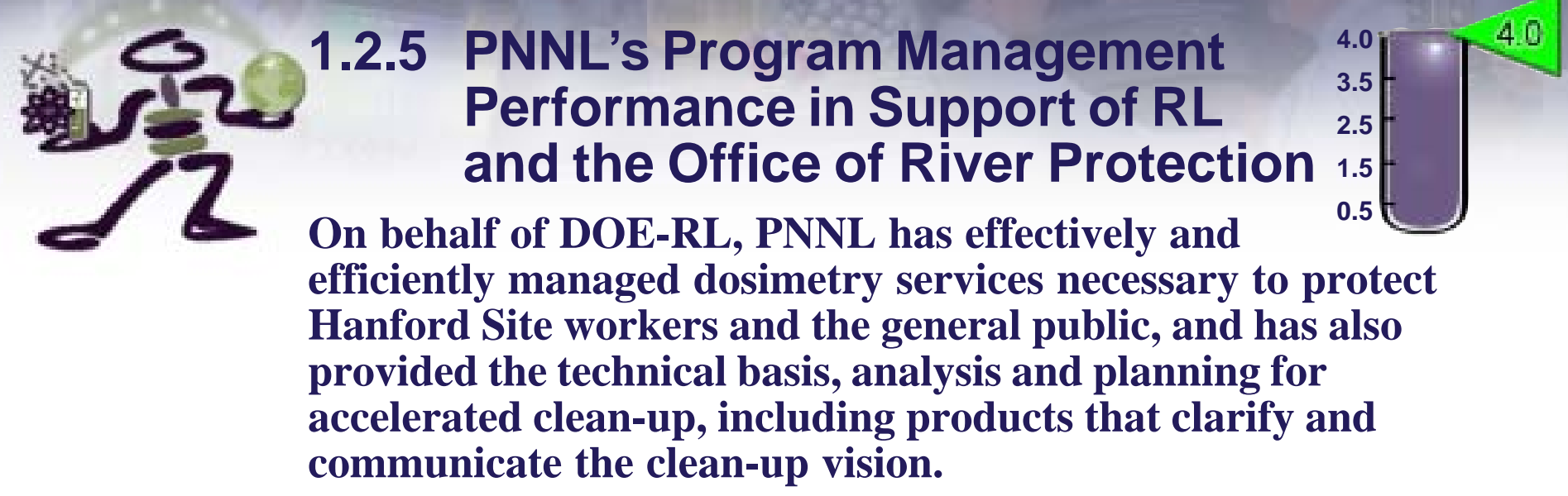

PNNL plays a critical role in ensuring the health and safety of Hanford Site workers, and in providing DOE and the other Hanford contractors with tools and expertise that accelerate Hanford's cleanup by integrating cost, schedule, and risk and thereby enabling informed Hanford Site cleanup decisions.

\section{State-of-the-Art Dosimetry Services and Support}

Through 40 years of providing radiation protection services to the Hanford Site, PNNL has developed the technical expertise and understanding of the Hanford radiological environment, DOE-RL and other Hanford contractor workers' needs, and regulatory drivers, to provide radiation protection services of the highest quality. State-of-the-art services include

- Dosimetry and occupational dose record management,

- Radiological instrumentation and calibration,

- Radiobioassay, and

- Non-destructive analysis.

In FY2003, neutron measurements required to ensure continued accurate dosimetry in the evolving PFP neutron environment were completed. The measurements will be used to ensure neutron dosimetry algorithms accurately measure doses for PFP workers as stabilization activities alter the neutron spectra of the radiation fields to which workers are exposed. Similar measurements were performed at spent nuclear fuel facilities that also have changing neutron radiation fields as a result of the remediation work being performed there.

PNNL provided the National Institute for Occupational Safety and Health (NIOSH) with more than 1,400 personal radiation dose histories for individuals applying for compensation under the Energy Employees Occupational Illness Compensation Program Act (EEOICPA). Dose histories and radiological exposure records were provided for individuals who worked at the Hanford Site from as early as the mid-1940's to the present day.

DOE-RL and the other Hanford contractors, recognizing PNNL's exceptional performance, continue to support PNNL as the provider of centralized radiation protection services. A team of Hanford prime contractors, led by Fluor Hanford, evaluated the cost of purchasing dosimetry services from PNNL and concluded that PNNL's dosimetry processing costs are appropriate for the quality and level of service provided and are within a commercially competitive range.

PNNL also provided a variety of uniquely configured and calibrated instruments designed to meet the unusual monitoring challenges faced by personnel on the Hanford Site. These tasks included low-energy photon correction factors for various container volumes for the RO-7 handheld radiation detector used at RPL gloveboxes, directional detectors for measuring ${ }^{241} \mathrm{Am}$, and high-range alpha contamination survey instruments to support PFP glovebox characterization activities.

\section{Hanford Solid Waste Environmental Impact Statement}

PNNL provided significant support to RL by reviewing, updating, and publishing the revised draft and final Hanford Solid Waste Environmental Impact Statement (HSW EIS). This EIS examined a wide range of treatment, storage, and disposal options for Hanford's radioactive solid wastes. A comprehensive team of scientists and technical support personnel evaluated both the nearterm and long-term environmental and human health impacts associated with the proposed actions. This high 
visibility project was greatly accelerated during FY2003 to prepare, in response to public and stakeholder comments, a revised draft EIS with a greatly expanded set of alternatives and waste types; to support the public involvement process; and to rapidly prepare and issue a final EIS and its associated records of decision (RODs). During the process, we gathered and addressed more than 5,000 public comments.

PNNL received a letter from Keith Klein applauding the success and expressing appreciation for the team's efforts: "I would like to express my sincere appreciation for the efforts PNNL employees have provided in support of the HSW EIS. Despite the aggressive schedule, uncertainties, and scope added during the development of the revision, the revised draft HSW EIS was issued for public review on April 11, 2003. The same work ethic continues to be shown during the development of the final HSW EIS. . . Their hard work, dedication and persistence in this task have indeed been admirable."
Preparation of the Final EIS (including conduct of the extensive NEPA Panel reviews by DOE-RL) was completed by the end of August 2003. Publication of the Final Hanford Solid Waste Environmental Impact Statement is currently on hold pending resolution of DOE-HQ and Department of Justice comments and issues. Upon concurrence from DOE-HQ, PNNL stands ready to rapidly produce the final HSW EIS and support the development of the RODs.

\section{In Summary}

In support of DOE-ORP, PNNL has conducted safety analyses of tank farm operations to ensure protection of Hanford site workers and the public; has performed technical analyses to optimize the deployment of accelerated waste treatment capabilities; and has provided planning for the integration of tank closure actions with other actions on the Central Plateau. These efforts were key to providing the technical basis, analysis, and planning for accelerating the cleanup of the Hanford Site.

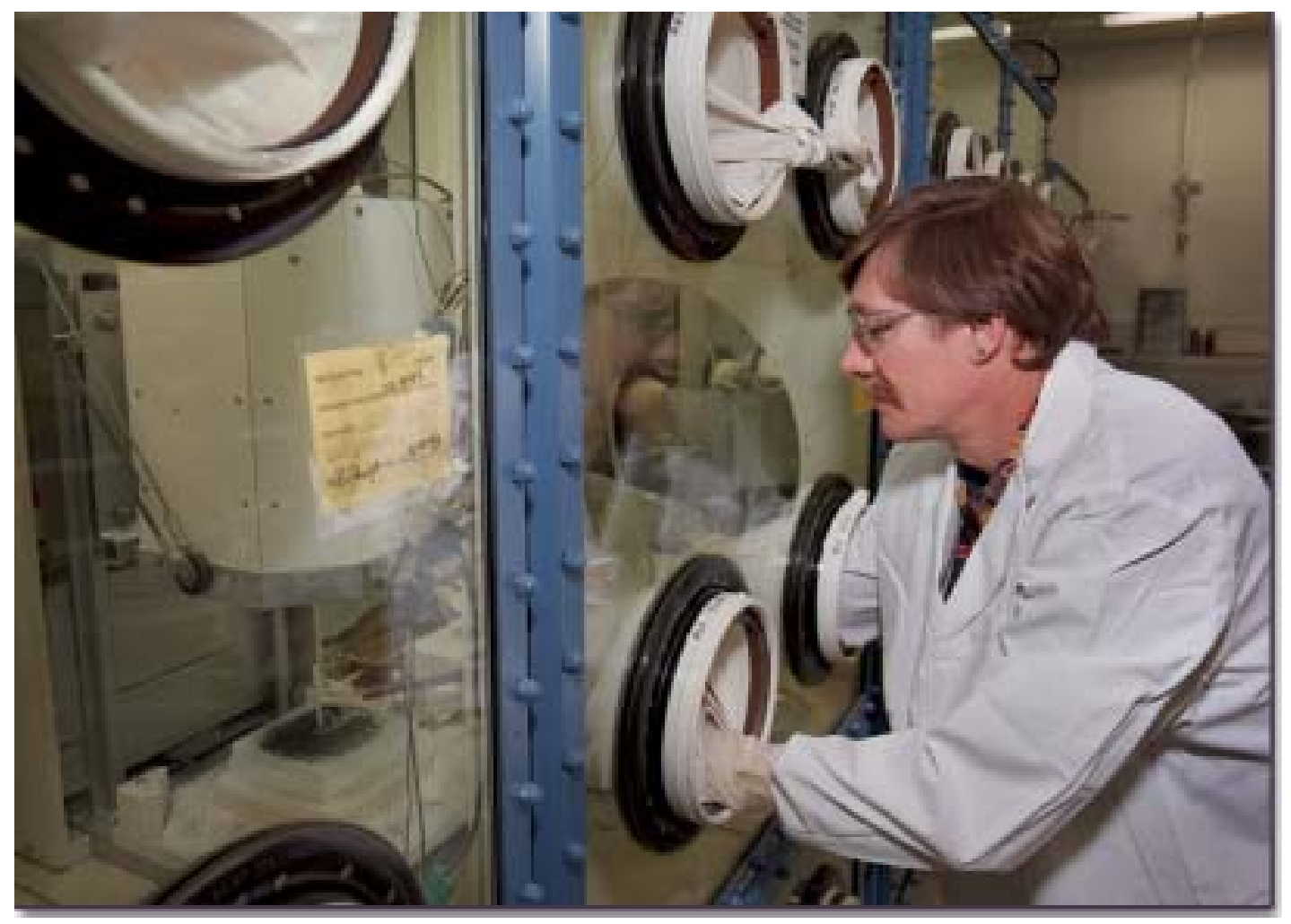

Figure 1.2.5. At the Radiochemical Processing Laboratory, researchers are discovering beneficial uses for radioactive materials using state-of-the-art equipment, such as this threecompartment glovebox facility. Radiation monitoring in RPL gloveboxes such as this is accomplished, in part, by a variety of uniquely configured and calibrated instruments, including the RO-7 hand-held radiation monitor. 


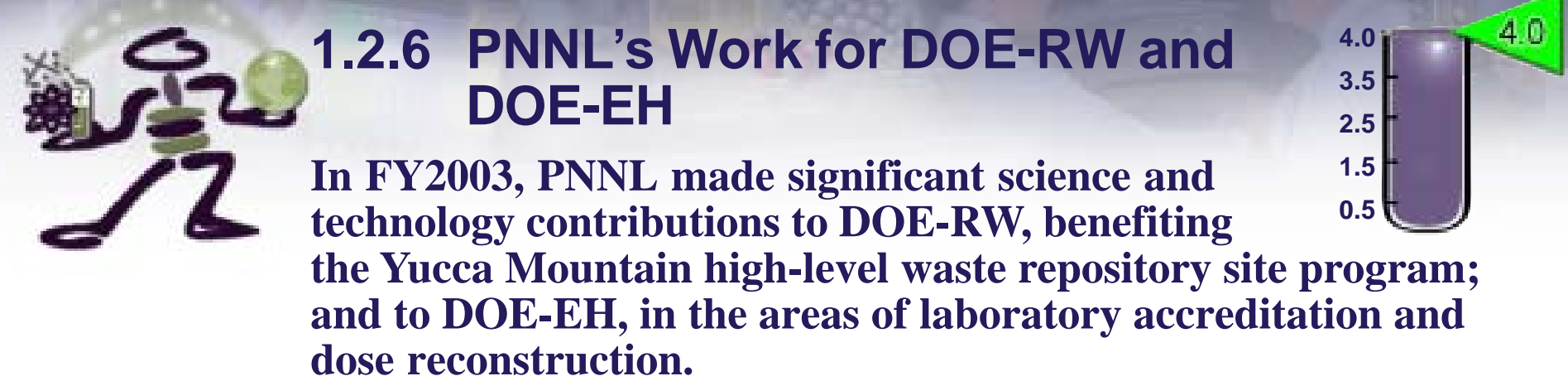

\section{Support to DOE-RW and the Yucca Mountain Repository}

In 2002, the President approved the Yucca Mountain, Nevada, site for the nation's first high-level nuclear waste repository, which is the responsibility of the DOE Office of Civilian Radioactive Waste Management (RW). PNNL's Radiochemistry Processing Laboratory has been helping to establish the basis for that decision for the last 17 years, establishing how Commercial Spent Nuclear Fuel (CSNF) could behave in an underground environment inside a metallic waste package for 10,000 years.

The qualified data generated have been used to develop the models for CSNF degradation, both dissolution and oxidation. These models, used first to support presidential site selection, will now help support the upcoming license application (LA) in December 2004. PNNL contributions to this Congressional LA milestone in FY2003 included completing additional waste tests, documenting results under strict program Quality Assurance requirements, and developing and reviewing licensing positions that will be submitted with the Application. Reviews and revisions of this material are ongoing.

Much of the data generated at PNNL and other laboratories in support of site recommendation and LA have been purposefully conservative, providing upper bounds on the waste form degradation rates. PNNL has proposed to make the case for a less stringent waste form licensing basis for Yucca Mountain through a new testing program to be jointly administered by the new RW Science \& Technology program and the project $\mathrm{M} \& \mathrm{O}$ contractor. The proposal includes tests that explicitly strengthen the basic science understanding of waste form performance. Results of the new tests would be used by RW to seek a licensing decision by the NRC in FY2007 and enable cost saving changes to the metallic waste package during repository operations.
Tests in four major areas have been proposed to determine the influence of a number of variables and mechanisms on waste form degradation and radionuclide release rates:

1. Realistic radiolysis conditions;

2. Chemistry of waste package material interactions;

3. Quantification of wetted surface area, particle generation, and colloid formation; and

4. Integrated CSNF testing in realistic humid and unsaturated repository conditions.

Each area has the potential of reducing either the modeled dissolution rate or release rate by at least a factor of 10 .

\section{Support to DOE-EH}

PNNL also continued to provide technical support to DOE's Laboratory Accreditation Program (DOELAP). The DOELAP program is critical to the DOE as it ensures consistency in personnel dose determination across the DOE complex. Part of this past year's efforts were spent managing the DOE Phantom Library, which develops reference radionuclide-loaded phantoms used to calibrate whole body counters throughout the country. Phantoms are also constructed for use in proficiency testing and accreditation of bioassay programs at DOE facilities. Constructing realistic tissue equivalent phantoms is technically challenging and has resulted in several patents at PNNL. Also, PNNL provides reference neutron radiations that are used in proficiency tests and accreditation of DOE external dosimetry programs. PNNL accomplishes this work through our National Institute of Standards and Technology-accredited secondary calibration laboratory for external dosimetry, DOE's only such facility. 


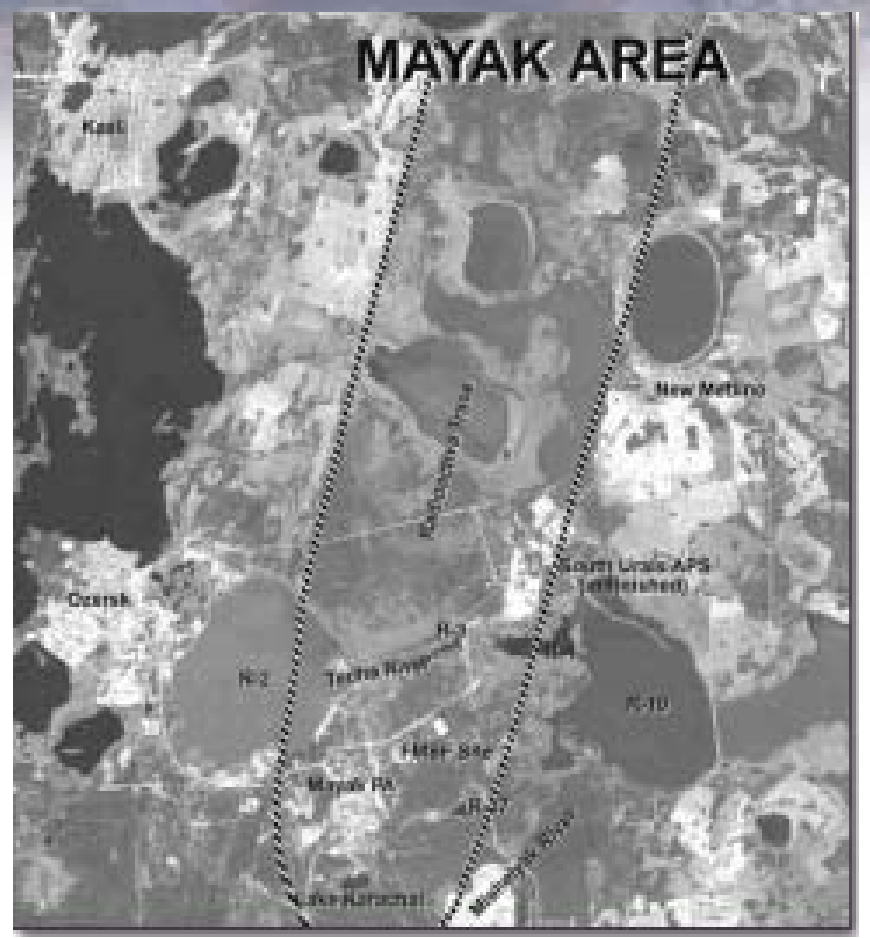

Figure 1.2.6. PNNL is contributing technical expertise to the Mayak Dose Reconstruction Study, which will develop understanding of the health effects to workers at the Mayak Production Association who routinely received greater occupational dose than U.S. workers.
In FY2003, PNNL provided technical expertise to DOEEH's Mayak Dose Reconstruction Project. This work is critical to determining past radiation exposures for former Russian workers at the facility, the Russian public living near Mayak, and the environment. PNNL staff have provided significant technical support since their initial participation, which began around May 2002. This support includes developing a Consolidated Task Plan by Russian and U.S. researchers to guide program deliverables, and preparing a dosimetry protocol by Russian and U.S. researchers to clearly specify the methods of dose reconstruction. The Russian Mayak workers received substantially greater radiation doses than comparable U.S. workers, particularly at the beginning of nuclear weapons development. The high doses, combined with extensive medical and occupational history documentation, may provide a unique opportunity to evaluate risks of protracted radiation exposure. 


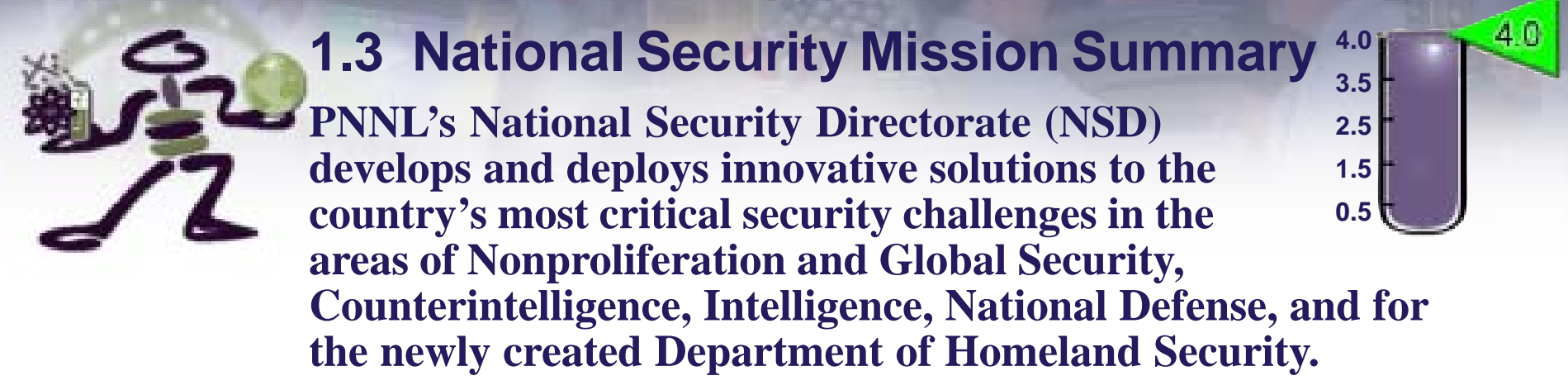

The modules in this section present examples of quality, relevance, and research program management from the perspective of PNNL's national security mission. Modules under this mission are summarized below into the categories of objectives 1.1, 1.2, and 1.4: Quality, Relevance, and Research Program Management.

\subsubsection{Peer Review. The Directorate Review Commit-} tee (DRC) of PNNL's NSD met at the Laboratory 23-25 June 2003 to conduct the annual review of programs and research activities. Committee members present were Carl Poppe (DRC Chair), Allen Atkins, Paul Greenberg, Joseph Kielman, and James Williams. As in June 2002, this meeting focused on the Directorate's response to the nation's Homeland Security challenges. In addition, NSD's DoD support was reviewed, and a special discussion session was held on issues surrounding closure of the 300 area. The Committee's summary included observations that funding has been maintained and even growing in certain areas, morale is high, NSD facilities and housing have been improved, the Homeland Security Initiative appears to be strong and is ahead of the curve in addressing new science and technology for Homeland Security, and significant opportunities exist for growth in the National Security arena.

1.3.2 Office of Defense Nuclear Nonproliferation (NA-20). In FY2003, PNNL marshalled fundamental science, environmental capabilities, and energy expertise to address critical U.S. national security challenges of detecting and preventing proliferation of weapons of mass destruction. Diversity in expertise among PNNL technical staff is key to effectively supporting the various elements constituting NNSA's Office of Defense Nuclear Nonproliferation (DNN). As the U.S. organization tasked with proliferation prevention, arms control, nuclear safety, and treaty verifica- tion, DNN continues to rely on PNNL to manage programs that result in a synergistic approach to global security. PNNL responded specifically to the needs of Nonproliferation Research \& Engineering (NA-22), International Nuclear Safety \& Cooperation (NA-23), Nonproliferation and International Security (NA-24), International Material Protection \& Cooperation (NA-25), and Fissile Materials Disposition (NA-26).

1.3.3 Office of Counterintelligence (CN). PNNL exploits its proven capabilities to develop an outstanding counterintelligence program that effectively provides value added support to DOE, NNSA, and the Intelligence Community. The ability of PNNL professional staff and scientists to conduct Counterintelligence activities, develop technologies, and deploy cyber security tools for DOE has positioned the Laboratory at the helm of projects critical to national security. The PNNL Counterintelligence Sector currently comprises four operational, analytical, and technical resource elements supporting DOE's centrally managed Counterintelligence mission to protect DOE/ NNSA classified and sensitive programs and information, personnel, and assets from foreign intelligence and international terrorist activities; and to detect and deter trusted insiders who would engage in activities on behalf of a foreign intelligence service or terrorist organization.

1.3.4 Office of Intelligence (IN) AND InTElligence WORK For OTHERs (IWFO). During FY2003, PNNL's Special Programs Office achieved major milestones in its nationally recognized analytical support for DOE's Office of Intelligence and related clients and in its leading-edge science and technology development for the Intelligence Community. Strong science-based intelligence analysis forms the foundation for PNNL to serve in lead roles on special programs for DOE's 
Office of Intelligence and other armed services and national intelligence agencies. Our analyses demonstrate a clear "value-added" beyond basic analysis, reflecting the nationally and internationally recognized technical expertise and experience PNNL brings to these tasks. PNNL staff were honored at a reception at CIA headquarters at Langley, Virginia, on March 31, 2003, where they were presented with National Intelligence Council Citations for Exceptional Performance and awarded National Intelligence Council Exceptional Performance Medallions for outstanding technical-analytical work performed over the last two years.

1.3.5 Department of Homeland Security (DHS). In FY2003, PNNL responded to the DHS/U.S. challenges related to national requirements for radiological, biological, and chemical terrorism countermeasures with science and technology related to sensing, monitoring systems, information analysis and technology insertion and evaluation. Key activities centered on the deployment of Radiation Portal Monitors for the Bureau of Customs and Border Protection, BioCountermeasures science and technology devoted to finding antibodies to detect chemical and biological agents, and development of technologies to monitor shipping containers for illicit nuclear and radiological materials.

\section{National Defense}

Projects in support of DoD continue to rank high in priority for PNNL and involve the broad application of scientific investigation across the Laboratory. Solving real-time problems such as aging weapons systems has allowed PNNL to engage experts in developing and deploying diagnostic and prognostic systems to help ensure system readiness in support of national security. PNNL's Holographic Imaging Radar Measurement System represents a new, state-of-the-art capability for radar signature management that is being developed for the U.S. Army's Aberdeen Test Center. Development and implementation of a Common Logistics Operating
Environment (CLOE) that uses information, communication, and other technologies will help to improve the Army's ability to sustain deployed forces while reducing logistics manpower and cost. The world's smallest catalytic fuel processing reactor system was developed by PNNL researchers to provide a low-watt power source for handheld wireless equipment, sensors, and other small but essential devices required by military troops. Important to the system is a revolutionary fuel reformer that enables the system to convert fuel and water into hydrogen-rich gas. Its success is stimulating development of larger units to meet battlefield power requirements. Creation of a new infrared spectroscopy technology base that includes basic signatures, detection technique development instrument design, analysis tools and photonics materials has broadened PNNL's business base in infrared technology. And, as stewards for the environmental restoration of Hanford and other DOE complexes, PNNL is transferring proven technologies to DoD sites with similar challenges. This effort will continue to be one of importance for DoD as it further reduces the magnitude of its infrastructure and support complex.

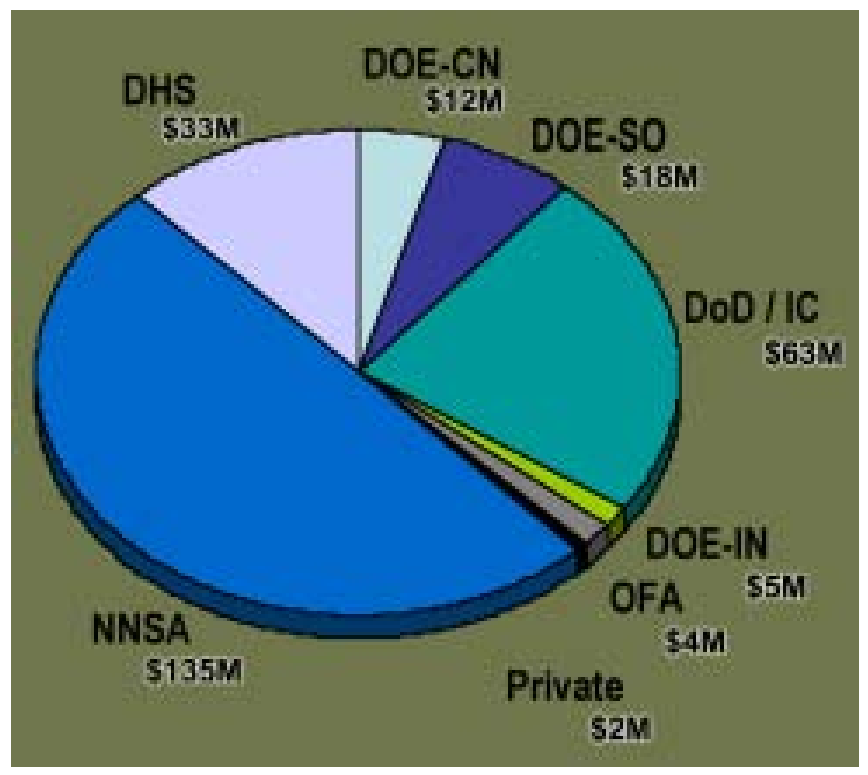

Figure 1.3. National Security revenue of $\$ 271$ million represented $46.7 \%$ of the overall Laboratory business portfolio in FY2003. 


\subsubsection{Results of National Security Peer Review}

\section{The National Security Directorate (NSD) Peer Review process validates the quality and direction of PNNL's National Security focused science and technology.}

The Directorate Review Committee (DRC) of PNNL's NSD met at the Laboratory June 23-25, 2003 to conduct the annual review of programs and research activities. Committee members present were Carl Poppe (DRC Chair), Allen Atkins, Paul Greenberg, Joseph Kielman, and James Williams. The June 2003 meeting again focused, as in June 2002, on the Directorate's response to the nation's Homeland Security challenges. In addition, NSD's DoD support was reviewed, and a special discussion session was held on issues surrounding closure of the 300 area.

The Committee review has evolved over the past several years, from one in which the sole focus was to examine about one-third of the $S \& T$ projects of the Directorate each year, to one in which reviewing strategic issues and challenges facing the Directorate has become as prominent as the $S \& \mathrm{~T}$ reviews. This has become especially important with the standing up of the Department of Homeland Security and the Laboratory's initiation of a program of activities to support the mission of the new Department. The Committee continues to review the S \& T portfolio of the NSD each year, albeit at a somewhat reduced pace from previous years.

\section{Committee Summaries of 2003 Annual Meeting}

\section{Action items from 2002 report}

In 2002 the Committee recommended improvement in two areas:

- To describe the connection between the Homeland Security Initiative and the Protection, Interdiction and Enforcement Technology Product Line portfolio, and

- To develop a one-viewgraph focus slide for Homeland Security.

The Committee believes that through the reorganization of the Homeland Security Program, the Laboratory has done a good job of dealing with these issues; the Committee is pleased to indicate its satisfaction that these issues are now closed.

\section{Strategic Planning}

NSD's current efforts, coinciding with the recent change in senior lab leadership, are especially timely and offer the opportunity to develop the future course of action for the lab in transition. The committee believes there is a great chance of success because of the relative simplicity of NSD's process. Those who will lead and participate in NSD's iterative process will be able to emphasize planning rather than process and format.

\section{DoD Programs}

The restructured NSD model for managing DoD programs reflects a new reality that harmonizes DoD projects and DOE science investments. The breadth and depth of the DoD projects at PNNL were impressive and involve a broad application of scientific investigation across the lab to solve customer needs.

\section{Homeland Security}

It is obvious that, in the year since the last DRC visit, the NSD has made excellent progress in establishing an inter-Directorate program at the laboratory as well as building a solid relationship with the Department of Homeland Security (DHS). The presentations and discussions focused on the Homeland Security Program Office, which was instituted early this year, and the Homeland Security Initiative, which began in the fall of 2002 . The primary goal of both is the creation and continued maintenance of a new Homeland Security (HS) business sector at the Laboratory.

A fourfold role was expressed for the program, which acknowledged that four distinct organizations have a voice in the future of this effort. These were to develop and nurture that long-term relationship with the DHS, to steward the DOE's developing science and technology agenda for homeland security, to coordinate and control business development of homeland security across all PNNL market sectors, and to ensure BMI participation and support. This comprehensive set of roles was ably reflected in the organization and functions for the HS 
Program Office. The DRC was impressed with that organization, which incorporated not only four DHS liaison functions that correlated with the DHS directorate structure but also included functions for regional outreach, PNNL linkage coordination, DOE lab coordination, coordination with the entire realm of federal agencies involved with homeland security, and BMI liaison. This organization will ensure that PNNL's Homeland Security efforts, to be directed by NSD, can be effectively managed.

\section{Area}

Since the last meeting of the DRC, DOE-EM has accelerated the schedule to clean up the 300 area, which houses a number of important nuclear and other capabilities needed by NSD programs. NSD, as well as other elements of the Laboratory, is faced with the problem of how to retain or reestablish those capabilities critical to important current programs as well as needed to move into new areas in support of Homeland Security.

The Committee believes that although NSD has jumped on the problem quickly, it has not yet developed the convincing argument as to why a new facility is needed.

The Committee recommends, as an action item, that NSD establish a Directorate team to develop the case for a new facility as described above, supporting multiple, synergistic customers that would replace and augment critical, needed capabilities lost because of the cleanup of the 300 area. The Committee will review progress in developing this plan during the coming year and at its next annual meeting.

\section{Summary}

The Committee remains very pleased with the excellent job Mike Kluse is doing in leading the Directorate and in the fine team he has assembled. All applaud the appointment of Barry Merrill as Deputy and the reapportioning of responsibilities at the top management level. It is a far more coordi- nated, streamlined, and enthusiastic unit than several years ago. Funding has been maintained and even growing in certain areas, morale is high, NSD facilities and housing have been improved, and significant opportunities exist for growth in the National Security arena. The appointment of Ned Wogman to run the Homeland Security Program is an excellent move, and Ned has done a great job in organizing his program to take advantage of the Laboratory's strengths and to match these to the new federal Department of Homeland Security. The Homeland Security Initiative appears to be strong, has the full support of the Laboratory, and is ahead of the curve in addressing new science and technology for Homeland Security. NSD should play a significant role in this emerging area.

The Committee was informed in several program discussions of the potential for significant growth. Although such comments are routine in reviews, the success of NSD in expanding its programs over the lifetime of this DRC suggests that the potential is real. This makes the need for a fully integrated strategic planning process, a more commercial perspective toward the research/development/production continuum, and broad succession planning critical.

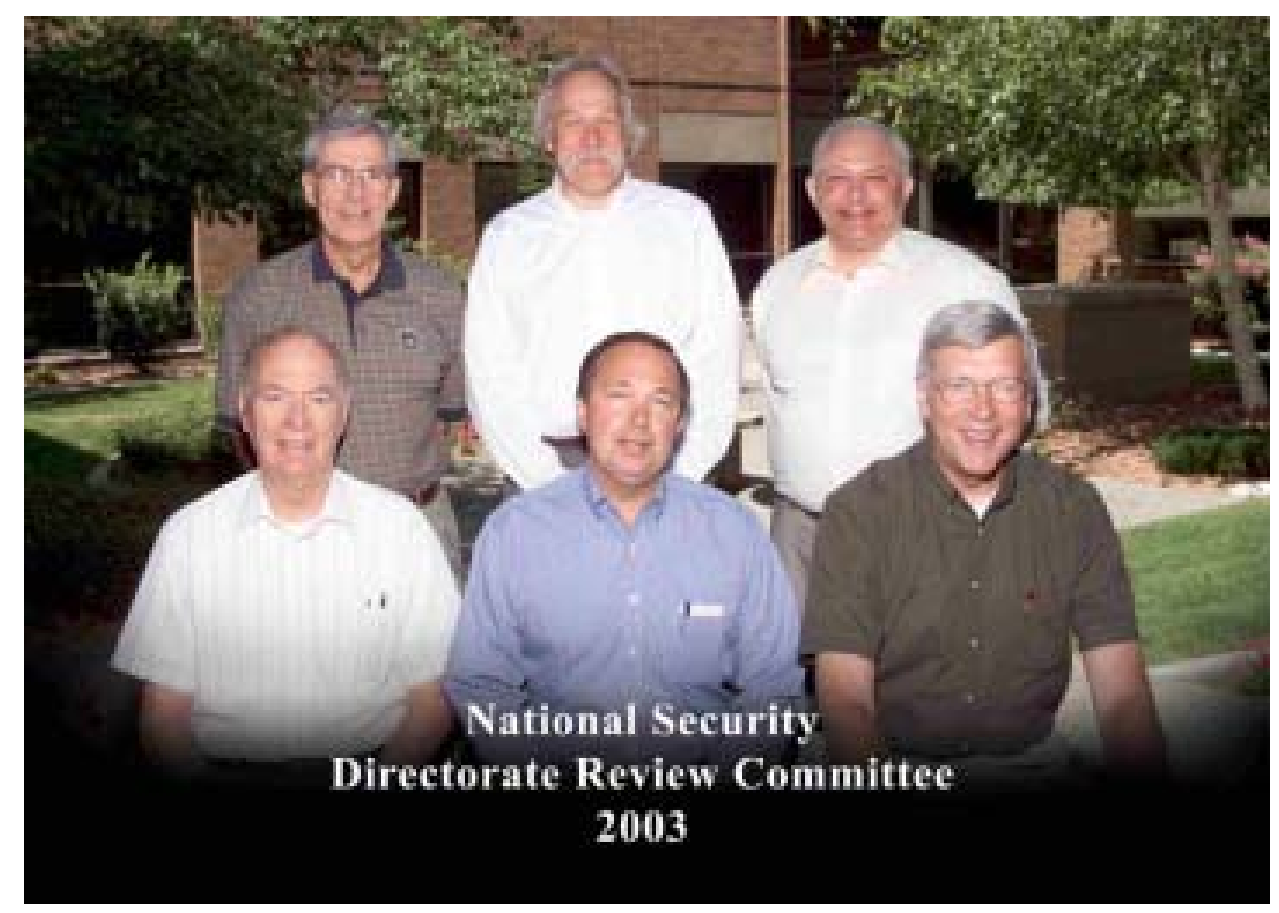

Figure 1.3.1. In FY2003, the National Security Directorate Review Committee focused on the Directorate's response to the nation's Homeland Security challenges, DoD support, and issues surrounding closure of the 300 area. 


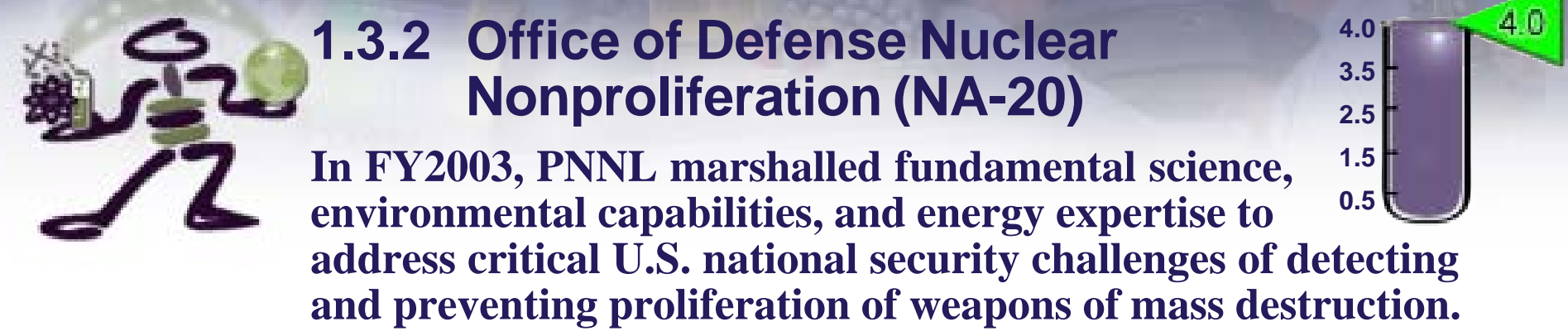

\section{Nonproliferation Research \& Engineering (NA-22)}

PNNL integrates knowledge of proliferation signatures provided by the Hanford legacy; experience from longterm missions in environmental monitoring; strong fundamental capabilities in nuclear, radiological, chemical and biological sciences; plus systems engineering and analysis expertise to develop innovative technologies for detecting and preventing the proliferation of weapons of mass destruction.

PNNL is rapidly becoming the premier DOE laboratory in infrared spectroscopy and its application to nonproliferation. PNNL completed version 7.2 of the IR Spectral Library, which was shipped to all government users in 2003 and now includes 272 data sets. During the past year, PNNL developed a chemical sensing field experiment for proliferation detection from ground and air platforms, which has multiple potential nonproliferation and homeland security applications. PNNL has also become the technical leader in mass spectrometry for nonproliferation applications, making excellent progress in developing a fundamentally new multi-element spectrometer than can simultaneously measure All the Signal, All the Time (ASAT).

\section{International Nuclear Safety \& Cooperation (NA-23)}

PNNL is applying science and technology to provide international nuclear plant safety and security, thereby furthering the United States' national security goals. PNNL provided a tenth year of outstanding program leadership of the highly successful program for international nuclear safety.

Major accomplishments in FY2003 included completing the In-Depth Safety Assessment (ISA) for the Russian Leningrad nuclear plant, installation of the Ukrainian Zaporizhzhya nuclear plant Unit 5 Emergency Control Room Simulator, and installation of security upgrades at the Ukrainian Zaporizhzhya plant. PNNL continued to provide outstanding project leadership for the Russian Production Reactor Nuclear Safety Upgrade Project, which consists of 21 projects to improve the near-term safety of Russian production reactors.

The International Emergency Management program managed by NA-23 helps ensure that foreign governments have adequate training, resources, and facilities to handle nuclear and other countrywide emergencies. PNNL's support of the rapidly expanding Emergency Management program has grown such that the majority of technical and contracting assistance is now provided by PNNL. PNNL purchased sophisticated videoconferencing systems for Russia, arranged nuclear emergency response training courses in Russia, and set up U.S. participation in Japan's annual nuclear emergency exercise at the Ohi nuclear plant.

\section{Nonproliferation and International Security (NA-24)}

PNNL is leading the assessment of technical policy options in a wide variety of nonproliferation and arms control problems, thereby enhancing global security through application of our broad range of science and technology expertise. PNNL staff continued to provide leadership to one of the world's largest and most important nonproliferation programs, the Kazakhstan spent fuel project. PNNL provided program management for the overall program to NNSA headquarters and led the feasibility study for the dual-use cask development for the canisterized spent fuel from the BN-350 Reactor in Kazakhstan.

PNNL continued to participate in the Agreement with the Russian Federation on the Exchange of Technical Information in the Field of Nuclear Warhead Safety and Security (commonly referred to as the WSSX Agreement). The WSSX Agreement engages U.S. and Russian technical experts in several areas that enhance the safety, security, and control of our respective nuclear stockpiles, nuclear nonproliferation initiatives, and nuclearrelated counterterrorism efforts.

PNNL assumed technical leadership for the State Department and NNSA-funded Kazakhstan International Nuclear Export Control Program, which ensures Kazakhstan does not export proliferation technology. 
PNNL continued to demonstrate leadership in the Nuclear Cities Initiative by leading thirteen new projects in the Russian closed cities.

PNNL expanded the geographic base of the Initiative for Proliferation Prevention program through a new project in Armenia and proposals in Uzbekistan, while continuing its traditionally strong emphasis on projects with the biological weapons institutes of the NIS.

\section{International Material Protection \& Cooperation (NA-25)}

PNNL continued to apply its 50 years of Hanford nuclear materials handling expertise to meet the challenges created by the nation's new concern for terrorism. PNNL provided program integration, technical, and management support to the Radiological Threat Reduction Program, successfully deploying physical security upgrades to twelve countries throughout the Former Soviet Union and Eastern Europe. Through PNNL's Second Line of Defense program, radiation fundamentals classes were conducted at the HAMMER site for U.S. Customs and Border Protection staff and Kazakhstan border enforcement officers. PNNL's Mayak project team completed comprehensive security upgrades on an additional 16 metric tons of $\mathrm{PuO}_{2}$ and $\mathrm{HEU}$, increasing the total to 28 metric tons of weapons-usable nuclear material that is now protected.

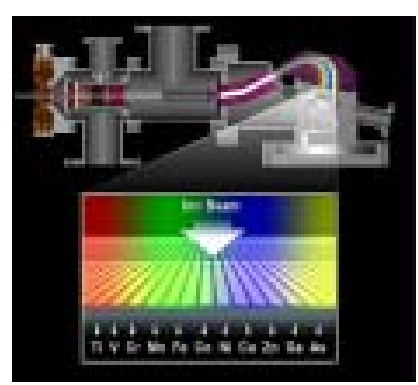

The Regulation Development Project completed seven MinAtom, one GAN, one Ministry of Transportation, and one Russian Shipbuilding Agency regulatory documents, held three regulatory workshops, and signed thirteen contracts covering the

Figure 1.3.2a. ASAT is a focal plane detector that can detect and measure a broad range of ion masses simultaneously and rapidly from a small sample.

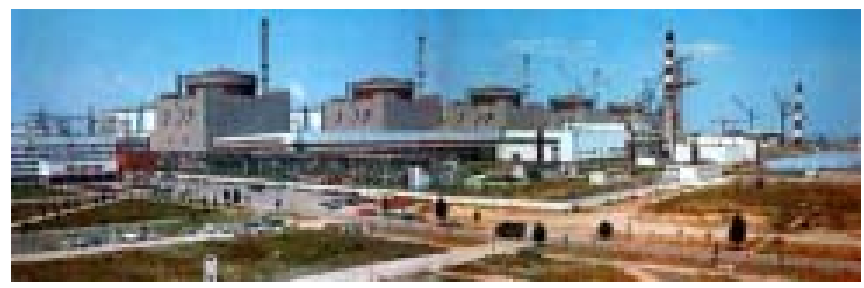

Figure 1.3.2b. PNNL completed installation of Unit 5 Emergency Control Room and security upgrades at the Zaporizhzhya Nuclear Plant in Ukraine.

\section{Fissile Materials Disposition} (NA-26)

PNNL is applying core capabilities in nuclear science and engineering, process technology, policy and regulatory analyses, and nonproliferation and arms control to enable execution of critical U.S.-Russia plutonium management and disposition agreements in the coming decade.

PNNL has continued to provide outstanding leadership for the Russian Federation Licensing/Regulatory Infrastructure Project for the Plutonium Disposition Program. PNNL awarded a contract to support a major revision to the primary plutonium disposition regulation, "General Safety Regulations for Nuclear Fuel Cycle Facilities," which had been developed under Russian Federation funding. PNNL was the lead author on a critical multi-lab paper that outlined the strategy for conducting monitoring activities both in Russia and in the U.S. to assure the plutonium disposition goals are met. This paper earned a Letter of Appreciation from the DOE Program Manager.

Battelle-PNWD also provided significant support to DOE via an 1831 contract as a subcontractor to Washington Group International on the design of the Pit Disassembly and Conversion Facility Project. In FY2003, DOE accepted the Preliminary Design Report, prepared in large part by PNNL.

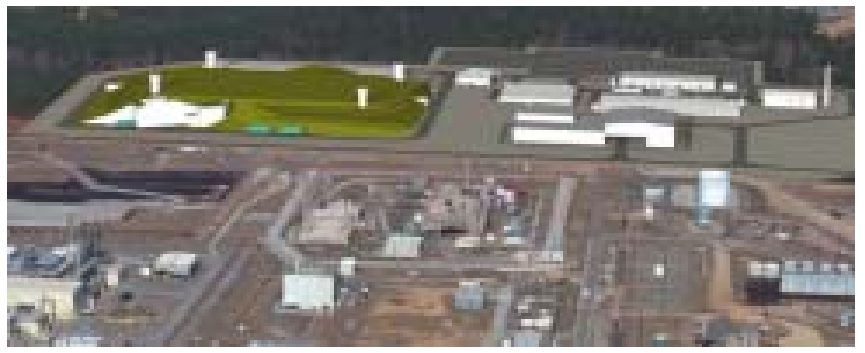

Figure 1.3.2c. The Pit Disassembly and Conversion Facility is shown here superimposed at the Savannah River Site.

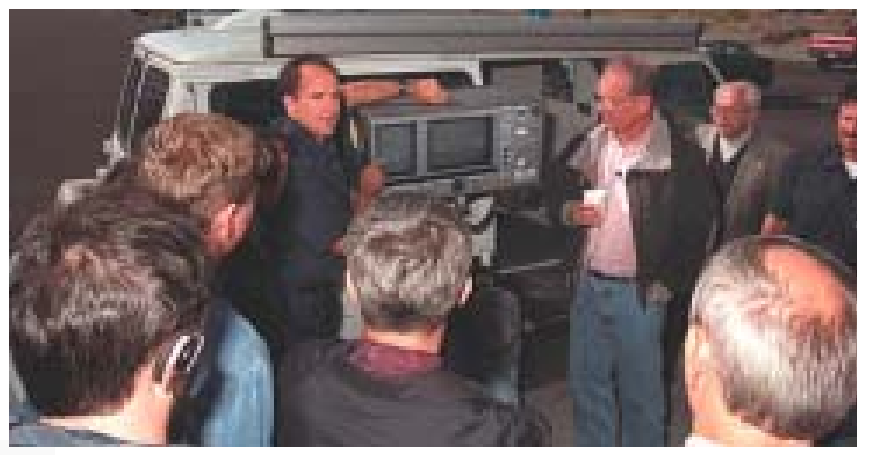

Figure 1.3.2d. PNNL conducted International Border Security Training at the HAMMER Facility. 


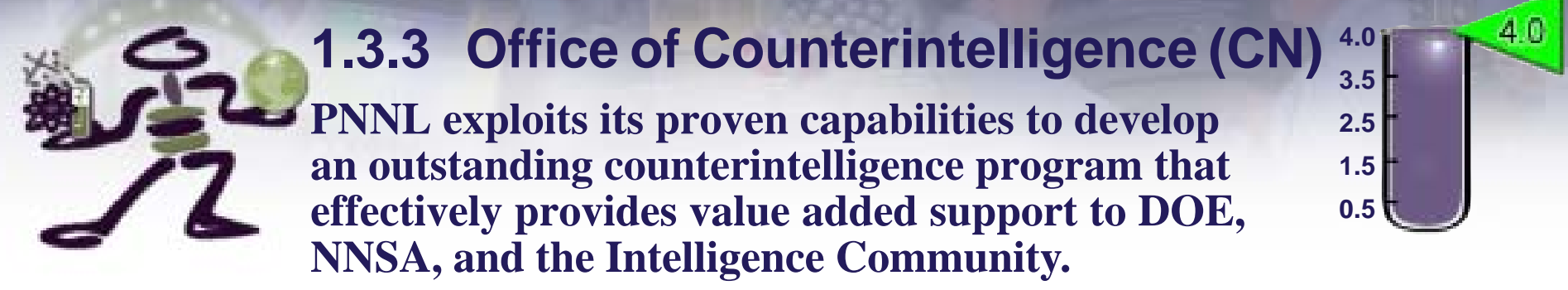

The PNNL Counterintelligence Sector currently comprises four operational, analytical, and technical resource elements supporting DOE's centrally managed Counterintelligence mission to protect DOE/NNSA classified and sensitive programs and information, personnel, and assets from foreign intelligence and international terrorist activities; and to detect and deter trusted insiders who would engage in activities on behalf of a foreign intelligence service or terrorist organization.

\section{Eight Highlights of FY2003 Performance}

The combined FY2003 budget for the four PNNL Counterintelligence Sector elements, at slightly under \$14 million, was the highest among all National Laboratories and field sites, as DOE/NNSA managers consistently recognize the PNNL Counterintelligence Sector as an important contributor to their national program goals and objectives as well as one of their most effective elements in the complex. In FY2003, significant accomplishments have been realized by PNNL Counterintelligence elements in the key function areas of staff training and awareness, analytical assessments, investigations, personnel evaluation, assistance to other agencies, responses to CI cyber threats, and special technical assistance related to the above:

- PNNL analytical resources were engaged in several special initiatives that helped DOE achieve goals to protect sensitive information and technologies from exploitation by hostile foreign intelligence services and/ or terrorist organizations. A comprehensive Hanford Site International Terrorism Threat Assessment, the first of its type from a local perspective, complemented PNNL's 2002 Foreign Intelligence Threat Assessment and is being considered by DOE/NNSA for use by other field sites.

- PNNL Counterintelligence staff regularly conducted special reviews to determine risk and vulnerabilities relative to staff interactions involving foreign national visits and assignments, unsolicited or suspect electronic communications, personnel evaluations, and other contacts and project matters.
- PNNL Counterintelligence Organization staff conducted and documented formal inquiries to resolve anomalous reports from PNNL staff and other sources related to incidents of Counterintelligence concern. A number of those matters have addressed DOE/NNSA's most complex counterintelligence issues, with subject matters of high national security significance. PNNL's performance in this regard is considered top quality by DOE/NNSA.

- All PNNL Counterintelligence elements organized and conducted multiple Counterintelligence training and awareness presentations for PNNL science and technology staff on hostile foreign intelligence collection, international terrorism, economic espionage, foreign travel, cyber threat, and other "audience specific" issues related to foreign interactions of risk potential. PNNL's use of the DOE/NNSA Counterintelligence Training Academy as a resource in the development of these presentations was noteworthy to the Laboratory's clients.

- PNNL's Counterintelligence Organization, by working with the OAC and other DOE Security and Counterintelligence units, maintained their on-going efforts to develop a new model integrating CI cyber expertise into traditional Counterintelligence investigative and analytical functions. This effort established an effective Counterintelligence cyber-relevant data collection and analysis process for $\mathrm{CI}$ threat review.

- The excellence of PNNL's Counterintelligence workforce has resulted in their selection to participate on a number of nation-wide working groups and forums. PNNL's Counterintelligence Program "model" and its identified best practices were also recognized by DOE/NNSA managers and exported to improve performance at other Counterintelligence sites in the complex and, internally at PNNL, to enhance the efforts of another Laboratory organization. 
- The Disaster Recovery Program (DRP) successfully began the establishment of a near-real-time backup and failover capability for the entire DOE/NNSA Counterintelligence record systems and networked communications. Both the national recognition and commendations for the IMAC-OAC, and DOE/ NNSA's decision to establish the DRP at PNNL, indicate the trust PNNL's program has developed across the complex and the respect earned for PNNL's effective, efficient project and program management.

- The PNNL IMAC program conducted regular analysis of network traffic to identify foreign open-source collection efforts, intrusion attempts, and other malicious activities against the DOE/NNSA complex. Technically, the OAC has achieved unprecedented thresholds in data volume and management, storing hundreds of millions of records in readily accessible databases. The OAC has established an exceptional level of coordination and cooperation among DOE/ NNSA sites that has enabled Counterintelligence entities to effectively harness the individual sites' CI-cyber efforts to monitor, identify, neutralize, and otherwise deter malicious activities involving national DOE/NNSA assets. The OAC coordinates with local site CI authorities and other national counterintelligence and computer security programs to identify and characterize threats against the national interests. The OAC publishes Information Intelligent Reports (IIR) for the Intelligence Community and collaborates with local and HQ elements of OCI to produce a variety of special reports.

In conclusion, the outstanding FY2003 performance of PNNL's professional staff and scientists to conduct Counterintelligence activities, develop technologies, and deploy cyber security tools for DOE has positioned the Laboratory as one of DOE/NNSA's primary locations to conduct projects critical to national security.

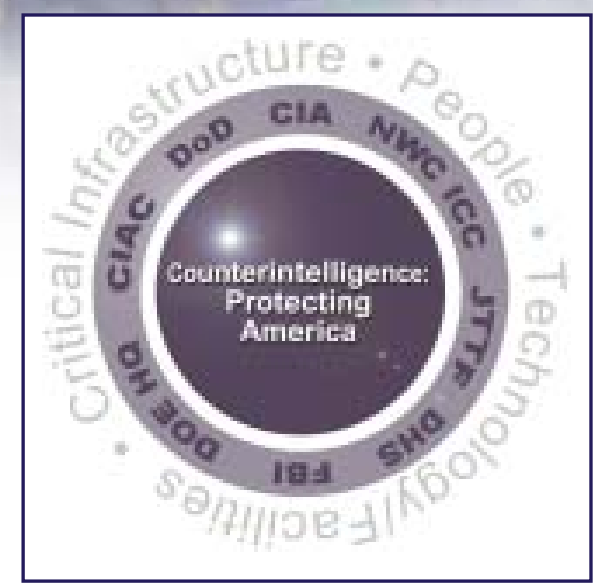

Figure 1.3.3a. Teamwork: A critical element of an effective counterintelligence program.

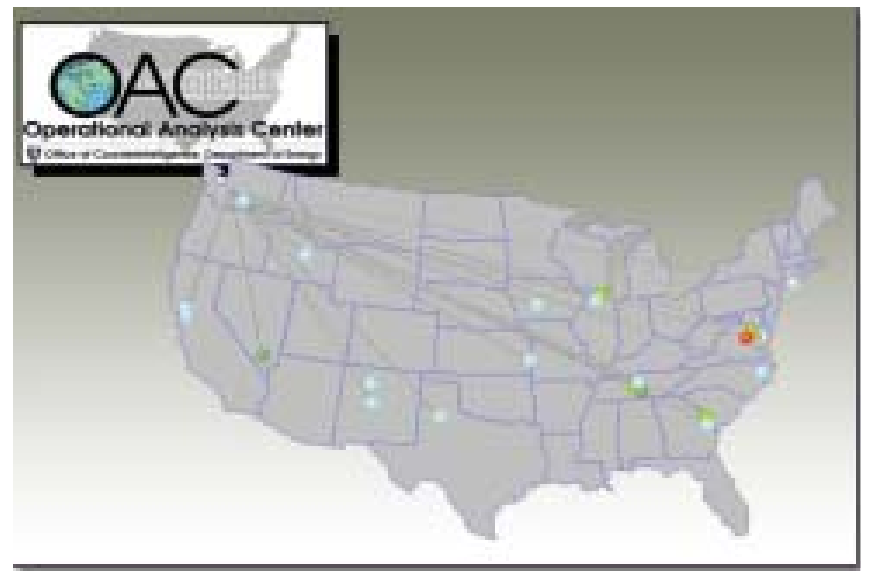

Figure 1.3.3b. PNNL is the hub of DOE/NNSA's cyber-Cl/CT program and an integral part of the US Intelligence Community's cyber-analytic efforts. 


\subsubsection{Office of Intelligence (IN) and Intelligence Work for Others (IWFO)}

During FY2003, PNNL's Special Programs Office achieved major milestones in performing nationally recognized analytical support for DOE's Office of Intelligence and related Intelligence Work For Others clients and in its development of leading edge science and technology products for the Intelligence Community.

\section{Science and Technology Developments Supporting the Intelligence Community}

Our work for intelligence-related organizations in the federal government draws on a wide cross section of Laboratory technical staff including:

- Information Science and Technology (research and application of our massive data set visualization software, network engineering technologies, analytical tools development, information operations)

- Nuclear Science and Engineering

- Nuclear and Chemical Environmental Forensics

- Radio Frequency/Electromagnetic Energy Engineering and Product Development

- Applied Development of Chemical and Materials Science and Engineering (including microchemical process engineering, catalysis research, chemical engineering, and materials development)

- Physics and Chemistry of Radioisotopes

- Development of Infrared Chemical Signatures of several hundred chemical compounds of interest to WMD nonproliferation issues

- Microrobotics and Related Mechanical Systems Development

- Micro/Nanomaterials Development and Application

- Electrical Power Systems Engineering including modeling and simulation of power grids and SCADA control systems engineering.

\section{Applied Technology Development for DOE/IN}

PNNL continued its longstanding participation in the DOE/ IN/Applied Technology Program during FY2003 with one new funded $R \& D$ project and a new task to provide staffing for a DOE/IN-USSOCOM shared Technical Liaison position in Tampa, FL.

\section{Intelligence Analysis For DOE/IN}

PNNL continued its well-established role providing high quality, quick-response information and analysis in support of DOE/IN and other senior DOE policy-makers including frequent intelligence briefings to NA-20 and other officials by our staff assigned to DOE/IN.

PNNL has agreed to place a highly experienced PNNL analyst currently on staff at DOE/IN in an Interdepartmental Personnel Assignment (IPA) to the new Terrorist Threat Integration Center (TTIC) as DOE/IN's representative to that integrative intelligence organization established by Presidential directive earlier this calendar year.

Our analyses demonstrate a clear "value-added" beyond basic analysis, reflecting the nationally and internationally recognized technical expertise and experience PNNL brings to these tasks.

\section{Intelligence Analysis for Others}

PNNL continues to have exceptional success in establishing problem centered cyber analysis cells supporting key IC client organizations within the Washington, D.C. area. 
These technical-analytical cells provide detailed technical analysis supporting IC, DoD, and Law enforcement and current problems of interest to protecting the assets of the federal government in areas principally related to Information Operations and computer network defense. The analytical cells capitalize on the PNNL-developed data visualization and information processing technologies and integrating these technologies with commercial and government proprietary capabilities into a "Discovery" network forensics analytical suite.

PNNL staff were honored at a reception at CIA headquarters at Langley, Virginia, on March 31, 2003, where they were presented with National Intelligence Council Citations for Exceptional Performance and awarded National Intelligence Council Exceptional Performance Medallions for outstanding technical-analytical work performed over the last two years.

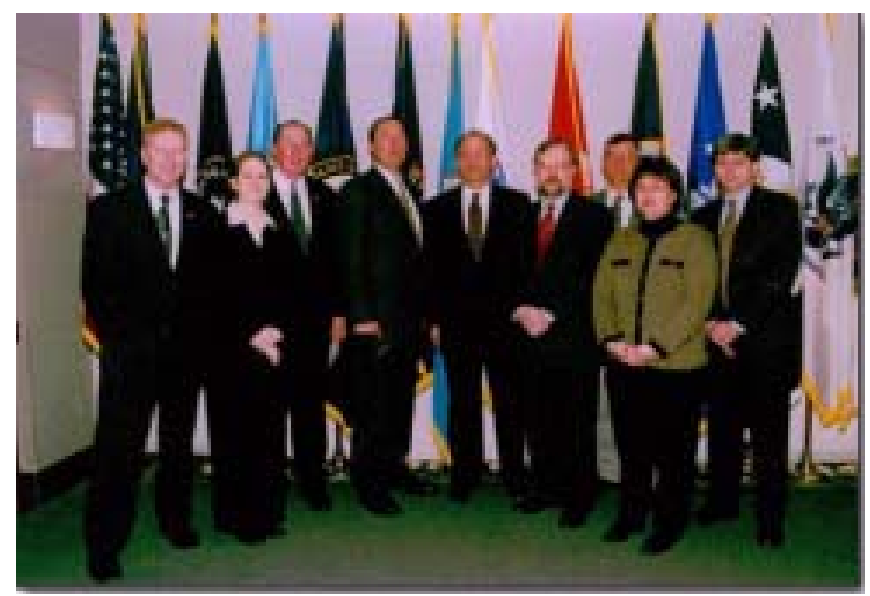

Fig. 1.3.4a. Awards being presented to PNNL staff by the Chairman Of The National Intelligence Council (NIC), Mr. Robert Hutchings together with Dr. Larry Gershwin, National Intelligence Officer For Science And Technology with the NIC. Mr. Mike Kluse, Associate Laboratory Director for National Security at PNNL was also present to congratulate the PNNL staff and represent PNNL during the ceremony. The PNNL staff being honored are assigned to both Richland and other PNNL offices in the Washington, D.C. area supporting various members of the Intelligence Community (IC).

In addition to providing staff on-site with clients for these analytical cells, PNNL continued providing recognized technical experts, at the specific request of host members of the IC, on temporary or permanent detail or Intergovernmental Personnel Assignment (IPA). Nine of our staff were so assigned during FY03 principally in the Washington, DC, region. We have also established an office supporting some of our clients in the San Antonio, TX area.

\section{Coastal Security Research at Sequim}

The National Security and the Environmental Technology Directorates at PNNL have jointly funded an LDRD research project at our Marine Sciences Laboratory during FY03 related to understanding how marine mussels accumulate contaminants from the environment and whether or not this natural chemical concentration mechanism can be a useful indicator for industrial processes. This capability of determining physical signatures in a coastal region has strong interest from the U.S. Government.

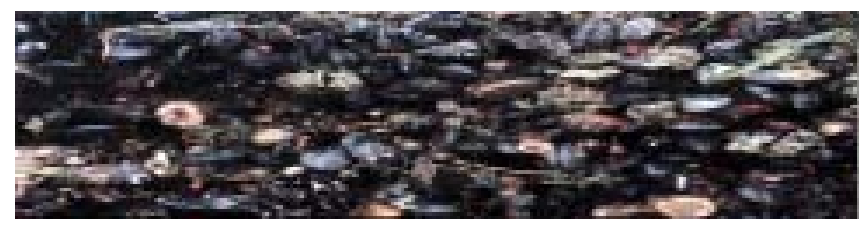

Figure 1.3.4b. Experimental validation of bivalves for the preconcentration of chemicals in aquatic environments.

This and related work is expected to lead to establishment of a Coastal Security Institute at the Marine Sciences Laboratory whose mission will be to support intelligence, national defense and homeland security efforts with uniquely qualified research and development focused on littoral regions.

\section{Infrastructure Improvements Supporting Work for DOE/IN and the IC}

PNNL recently completed a 4-fold increase in SCIF space and related secure broadband communications capabilities. We are in the final stages of having an approved installation for SIPRNET secure DOD communications connectivity in addition to the DOE/SEAS capability. Through these Laboratory investments we continue to support DOE and the Intelligence Community (IC) through expert operation of these essential facilities. We received interim accreditation for the enlarged SCIF in January 2003, and final accreditation in May 2003 and we also received high marks for our security operations during the Inspection and Evaluation by DOE/HQ conducted in May 2003. 


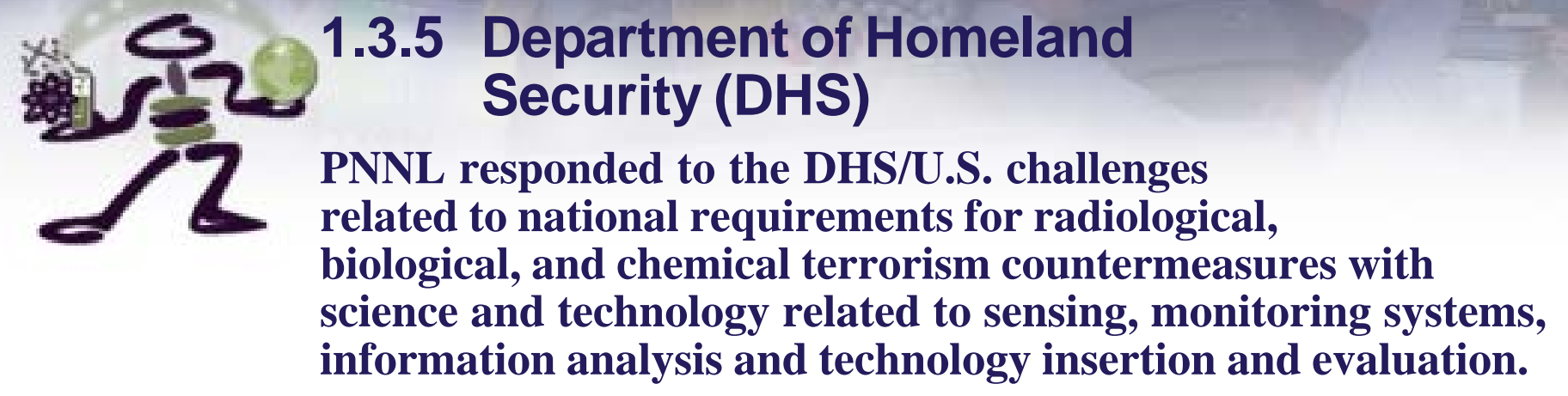

\section{Radiation Portal Monitor Overview}

Since January 2002, PNNL has been providing direct scientific and technical support to the Bureau of Customs and Border Protection (CBP) in their post-9/11 mission of interdicting radiological and nuclear weapons or their components. Using a systems engineering approach, potential threat 'vectors' (methods or avenues for the introduction of threat-related materials) were found and commercially available equipment suitable for CBP's radiological threat interdiction mission was identified. Beginning as primarily a consulting effort to provide expert opinion regarding technology options and operational insertion methods, the project rapidly grew to include the deployment of Radiation Portal Monitors (RPMs) at more than 240 CBP facilities across the United States. In all, more than 1900 RPM systems will be deployed.

PNNL has developed specifications that enhance the generic nature of an RPM to include the elements necessary for effective insertion into the CBP inspection environment. Specifications for the PNNL-borne concept of a nationally-integrated radiation detection network were developed. When complete, the CBP will have the real-time ability to monitor and control RPM systems nationwide, to observe and record their operation, and to remotely alter alarm-threshold values. This network will be the largest radiation sensing system of its type in the world.

The RPM project is a large and complex effort, with deployment activities accounting for a majority of the work. PNNL fields teams to support all aspects of deployment, from site surveys through design, installation, and commissioning. In addition, PNNL is providing interim deployment support to ensure the deployed RPM systems continue to operate effectively.

Under CPB direction and support, PNNL is researching methods to fully exploit all of the information available from a portal monitor. Many forms of cargo are naturally radioactive and create a large number of nuisance or Naturally Occurring Radioactive Material (NORM) alarms. The methods that result from PNNL's research may ultimately allow the RPM to 'ignore' sources of NORM and thereby increase the sensitivity of the portal without slowing the flow of commerce.

\section{BioCountermeasures Overview}

PNNL's BioCountermeasures science and technology program spans the gap from determining specific proteins expressed by pathogens, finding antibodies to detect chemical and biological agents, environmental sampling and detection of pathogens, the decontamination of biological and chemical agents, to evaluating and improving urban indoor and outdoor dispersion models. State-ofscience mass spectrometry is identifying proteins specific for Yersinia pestis, yielding protein signatures for detection. An antibody library expressed on the surface of yeast is being mined to provide high affinity antibodies for botulinum toxin and other biological and chemical agents. An integrated bioprocessing system using automated fluidics and renewable surfaces is being developed as a universal sample preparation platform for pathogen detection. A decontamination technology is being developed and then demonstrated that uses an enzyme-based fog technology to decontaminate chemical and biological agents. Finally, field studies are being conducted to provide quality assured data sets for evaluating and improving urban indoor and outdoor dispersion models used to simulate dispersal of potential toxic agents in urban atmospheres.

\section{Containers Monitoring Overview}

PNNL is developing an Instrumented Container system to detect illicit nuclear and radiological materials concealed in maritime cargo containers. The system, based on rugged and inexpensive gamma-ray and neutron sensors mounted in container walls, and wireless 
communications that transmit measurement data to a central location as the ship transits to the U.S., will provide primary screening en route to the U.S., an effective alternative to container inspections performed after the ship arrives in port. Such an approach to maritime cargo monitoring takes advantage of the relatively long transit times of cargo destined for U.S. ports (typically longer than a day for maritime cargo), and has the advantages of improved sensitivity to nuclear and radiological materials, reduced primary screening burden on port operations, and early warning of suspect containers.
A fundamentally different type of gamma-ray sensor based on optically stimulated luminescence materials (OSL) is being developed to meet the cost and power consumption requirements of unattended long-term monitoring for containers. Sensitivity studies, the development of appropriate data analysis algorithms, and the integration of wireless communication with our prototype radiation sensors are key components of this nuclear smuggling project.

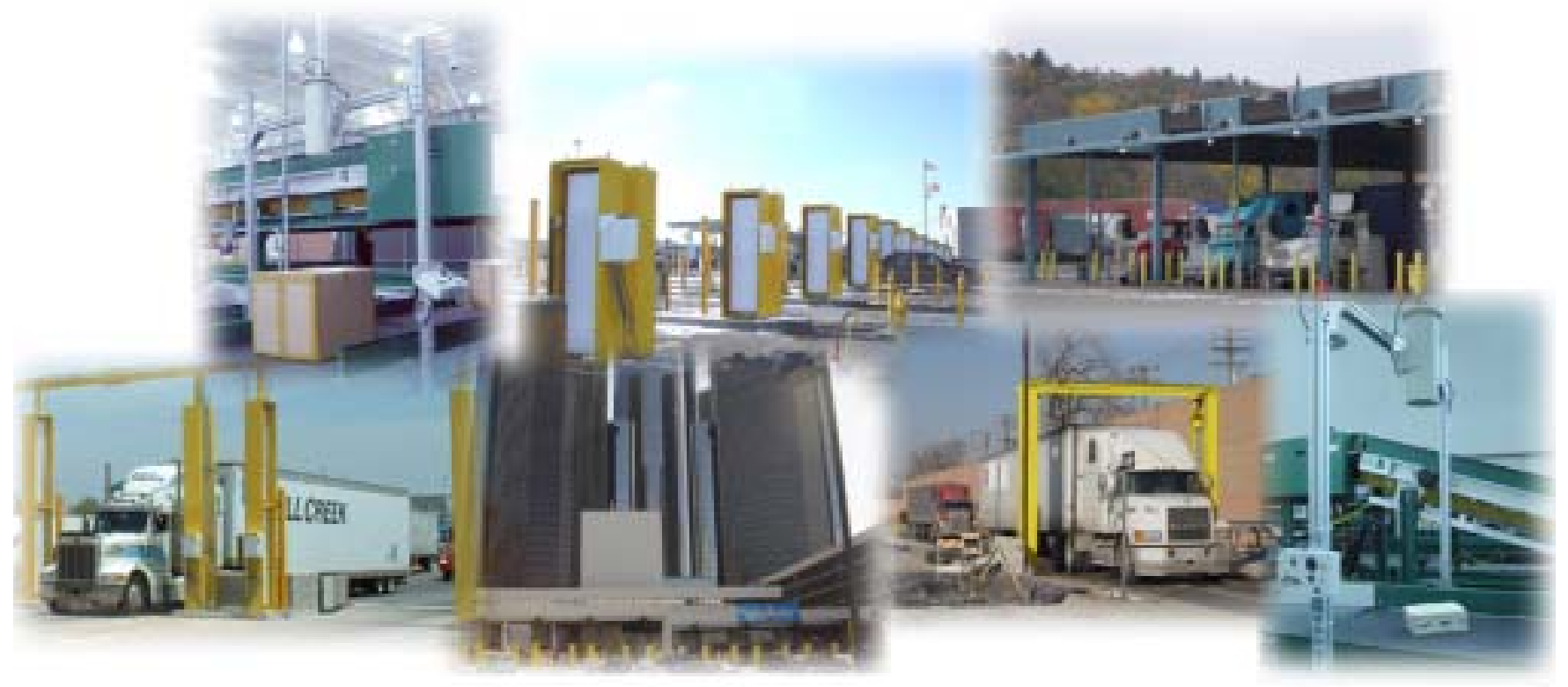

Figure 1.3.5. Radiation Portal Monitors are being used to interdict radiological and nuclear weapons or their components. 


\subsection{Energy Mission Summary}

In support of national priorities and DOE Energy missions and program plans, PNNL researchers continue to demonstrate leadership, technical quality, innovation, and impact in converting, transporting, and using energy. Our dedicated technical staff have worked with senior PNNL management and DOE program leads, and formed partnerships with industry to deliver high-quality products and services.

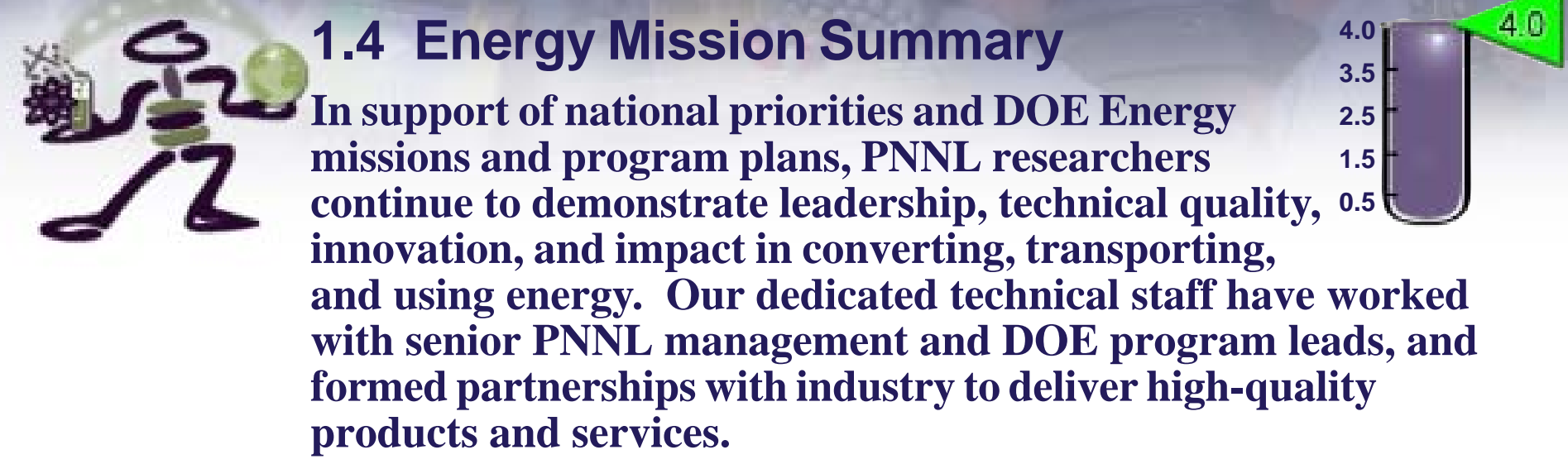

PNNL is delivering science and technology solutions, analysis and strategy to help bridge the gap between today's energy systems and tomorrow's hydrogen economy. Our contributions in these areas support efforts to maximize energy efficiency, increase the use of renewable energy, address environmental concerns, ensure economic feasibility of energy options, and develop energy policies for the future.

The modules in this section present examples of quality, relevance, and research program management from the perspective of PNNL's energy mission. Modules under this mission are summarized below into the categories of objectives 1.1, 1.2, and 1.4: Quality, Relevance, and Research Program Management.

1.4.1 Peer Review. The Energy Science and Technology Directorate (ESTD) Peer Review process validates the quality, relevance, and directions of PNNL's energy science and technology. In FY2003, peer reviews within the Directorate were structured to be more strategic than tactical, to enhance the evaluation process of PNNL's energy science and technology. This approach enabled more timely feedback on Directorate progress in key business areas and four Initiatives.

1.4.2 Advancing Developments in Distributed Energy AND ENERgy Systems. In FY2003, PNNL continued to demonstrate leadership in the development of advanced communication and control technologies to benefit a highly connected electricity grid and distributed energy systems. PNNL is "bringing the energy system into the information age" through research in high-speed computation and simulations that will help transform the energy system into an information-rich, transactive network that is intelligent, robust, reliable, and secure. Our capabilities in modeling and computation, grid reliability, materials science, fuel cells and other innovative energy technologies, and economics and policy analysis, underpin this transformative work. We have made key contributions to Office of Building Technology strategic objectives through projects such as the Building Energy Codes, Emerging Technologies, Building Systems, and EPAct Standards. High quality products, important strategic planning assistance, critical technical assistance, and innovative approaches to achieving DOE's strategic objectives are provided through these and other projects. We also provided extensive support and leadership to DOE in the development of a proposed code change that included a significant Residential IECC Code Change (RICC) proposal.

1.4.3 Paving the Way for a Transition to a Hydrogen ECONOMY. PNNL is working closely with other national laboratories and industry partners to develop science and technologies solutions that will bridge the gap to a hydrogen economy by addressing specific areas core to the national hydrogen program and establishing centers of excellence in hydrogen storage materials and safety. In 2003, we were assigned leadership of DOE's hydrogen safety program. We are conducting integrated analysis and evaluation of hydrogen systems as part of the Hydrogen Fuel Initiative. The first tasks will be to develop guidelines for future hydrogen projects and to establish a Hydrogen Safety Panel. In September FY2003, PNNL and Los Alamos submitted a proposal to become one of six hydrogen storage centers, focusing on chemical hydrides, an approach that builds on our strong capabilities in materials and catalysis. 
1.4.4 Advancing Transportation Technologies for High-EFficiency TransPortation Systems. PNNL is developing advanced transportation technologies for essential power systems, lightweight materials, and emissions aftertreatment. Our focus on specialized materials and fuel cell technologies are helping increase the reliability and decrease the cost of fuel cells for distributed energy and high-efficiency transportation systems.

1.4.5 Creating InNovative and Economically Viable BioBased Products and Processes. In FY2003, PNNL made excellent progress in developing biobased products and processes to displace imported petroleum and implement integrated biorefineries. The major goals of the Office of the Biomass Program (OBP) are to reduce dependence on foreign oil and establish integrated biorefineries that produce a cost-effective combination of products, fuels, and power. In FY2003, PNNL continued innovative, high-quality research, and worked effectively with industry to help DOE create economically viable biomass technologies.

1.4.6 Developing Technologies for Clean and EfFicient Power Generation. PNNL is advancing the objectives of the DOE FutureGen Initiative though the expansion of the Solid-state Energy Conversion Alliance (SECA) and regional demonstrations of carbon management technologies. FutureGen will involve designing, building, and operating the world's first near zero-emissions coal-fueled power plant. If it fully materializes, this project will be one of the most significant accomplishments across the DOE energy program offices in decades.
1.4.7 Support of DOE EnERgy ANd National Security Missions with Advanced Nuclear Science and TECHNOLOGY. PNNL is sustaining and building national capabilities in nuclear science and engineering in support of the nuclear component of national energy and security goals. In FY2003, building on our capabilities in low-level radiological detection, we began focusing on the concept of establishing a Radiological Detection and Control Center. We also provided critical science and technology support to the Nuclear Regulatory Commission in its efforts to re-license operating nuclear plants. By applying our materials science capabilities, our researchers are developing techniques to detect and prevent radiation damage to reactor core components. These solutions are helping extend the life of the nation's nuclear reactors, which provide 20 percent of the U.S. energy needs.

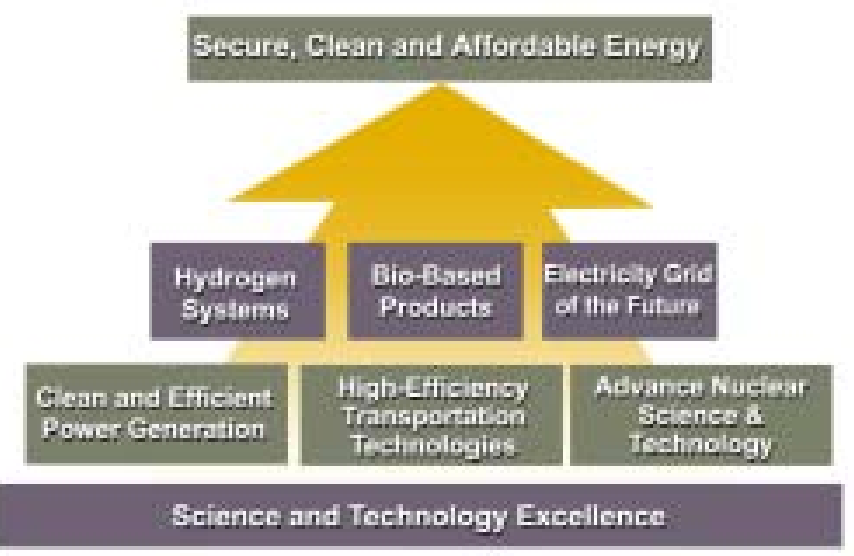

Figure 1.4. Pacific Northwest National Laboratory supports DOE Energy Missions in providing science and technology solutions for secure, clean and affordable energy. 


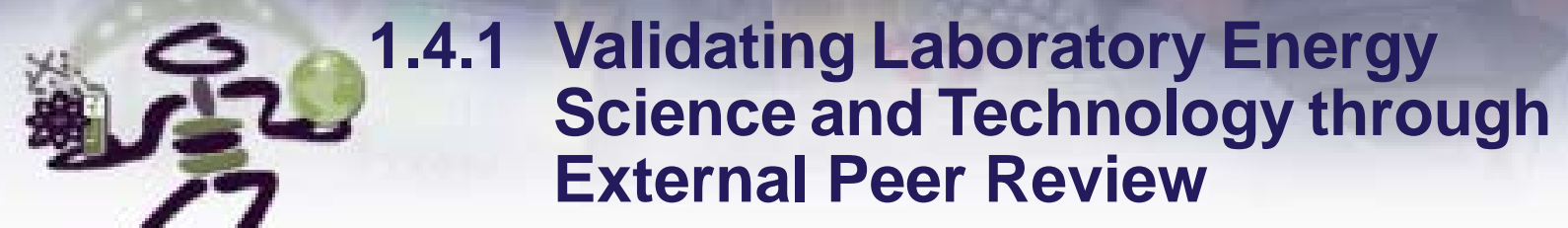

The Energy Science and Technology Directorate (ESTD) Peer Review process validates the quality, relevance, and directions of PNNL's energy science and technology. In FY2003, peer reviews within the Directorate were structured to be more strategic than tactical, to enhance the evaluation process of PNNL's energy science and technology. This approach enabled more timely feedback on Directorate progress in key business areas.

\section{Directorate Review Committee}

The ESTD Review in May 2003 used a hierarchical approach to evaluate the three main business areas: 1) Energy Systems \& Engineering, 2) Information Sciences \& Engineering, and 3) Materials Science. We asked the committee to focus on science and technology issues and to evaluate the Directorate's strategic objectives in light of the emerging Office of Management Budget (OMB) investment criteria for federally sponsored research and development. This system focuses on three factors: Quality, Relevance, and Performance, each of which, with their associated criteria, were evaluated Outstanding, Excellent, Good, Marginal, or Unsatisfactory.

The Directorate Review Committee, comprising leaders from both industry and academia in the three main science and technology areas stewarded by the Directorate, was chaired by Jesse Berst of the Athena Institute, and included Mike Davis of Avista Labs, former Energy Secretary Don Hodel of Summit Energy Group, James Foley of Georgia Institute of Technology, Edward Fox of Virginia Tech, Larry Dalton of the University of Washington, and Toni Grobstein Maréchaux of the National Research Council of the NAS. Nuclear business areas were reviewed by John Ahearne of JRFA, Inc. during his visit to PNNL May 23.

Reviewers received reading material provided in advance, presentations by staff members, and held discussions during the review. The three main business areas were evaluated individually, then rolled up into a directorate evaluation. The ESTD review committee said, "ESTD is an exceptional part of an exceptional organization. It is an enormous asset to the region and to the country as a whole. We believe ESTD is doing a first-rate job."
Overall, the committee rated the research excellent, the relevance good, and the performance excellent. Management was congratulated for creating an atmosphere that produces diligent, hard work and for making good strategic use of EMSL. The committee believed ESTD has the fundamentals in place for enormous success in the years to come and commended the Directorate's major effort in the past year to create a cohesive vision and mission. The next steps are to align strategic thrusts with that vision and to choose a small number of priorities.

Recommended areas of improvement included defining a Directorate signature capability, increasing efforts to expand our customer base, and building new strategic partnerships. The Committee recognized ESTD could build on Laboratory signature facilities to create a signature capability. The committee also suggested building constituencies among legislators, government officials, and regional and business groups to support and fund key initiatives.

\section{Initiative Peer Reviews}

\section{Advanced Nuclear Science and Technology Initiative (ANSTI)}

The ANSTI review committee found this LDRD work impressive and exceeding expectations. They applauded refocusing the initiative on broad capability development applicable to many missions, especially the cross-cutting capabilities critical to Homeland and National Security. Feedback noted

- Significant progress on the Integrated Nuclear Strategy (INS), although some redefinition and business development work remains; 
- Since business conditions can change, ANSTI leadership and Laboratory management need to continue to test markets and reevaluate direction and alignments; and

- ANSTI's focus on developing the Radiation Detection Analysis Center (RDAC) in close coordination with both the PNNL Homeland Security Program Office and the Homeland Security Initiative is excellent and could result in an important investment in facilities enabling PNNL to maintain, even increase its nuclear capabilities and business. Developing a long-term RDAC management strategy should be a near-term priority for PNNL leadership.

\section{Bio-Based Products Initiative (BBPI)}

The BBPI Advisory Committee met in August 2003 to review technical and business progress, suggest how to move from internal to sustained external programmatic support over the Initiative's remaining two years, and discuss the future of PNNL's bio-products program. The committee agreed that

- Scientific and technical progress has been excellent;

- Mature chemical catalysis research no longer needs LDRD support;

- Fungal biology and biotechnology research area has made excellent progress but requires further LDRD investment in fungal genetics, proteomics, and chemostat capabilities; and

- The initiative successfully built a central role for PNNL in the DOE-EERE Office of Biomass Programs (OBP) and made significant progress toward building distinctive signature capabilities in chemical catalysis and fungal biotechnology. The initiative also is building a strong relationship with DOE OBP, as evidenced by DOE's offer to assign a PNNL staff member at DOE headquarters to help plan the program. Strong OBP support validates that the Initiative is on track in aqueous phase chemical catalysis.

\section{Carbon Management Initiative}

The Carbon Management Initiative Advisory Committee met via telecom in July 2003 to review Initiative progress and provide advice on direction and strategy for FY2004 and beyond. The Initiative managers and PIs summarized the vision, strategy, technical approach, and progress. The committee, recognizing PNNL as a center of excellence in carbon sequestration, noted management's excellent performance in marketing and business positioning analysis, particularly in relation to government, academia, and other national labs.

\section{Energy Systems Transformation Initiative}

The Energy Systems Transformation Initiative Advisory Committee met in August 2003 and reported excellent technical and scientific progress. The technical projects have developed fundamental science and technology for understanding how the power system functions and how new technologies can be used to improve it. The business side has also been active, using DOE's reorganization to incorporate the GridWise ${ }^{\mathrm{TM}}$ concept and brand, thus demonstrating DOE's recognition of PNNL as a thought leader in this area. The committee felt contributions to the development of the DOE Office of Electric Transmission and Distribution were outstanding. Recommendations included

- Developing and delivering a better articulated initiative transition plan - in particular, more specifics on how technical and business development will proceed. The Committee recommended that the plan be available for review in early December;

- Including specific outreach activities and objectives in the initiative transition plan; and

- Including a policy analysis expert to help understand how to transition the energy system and how PNNL can help.

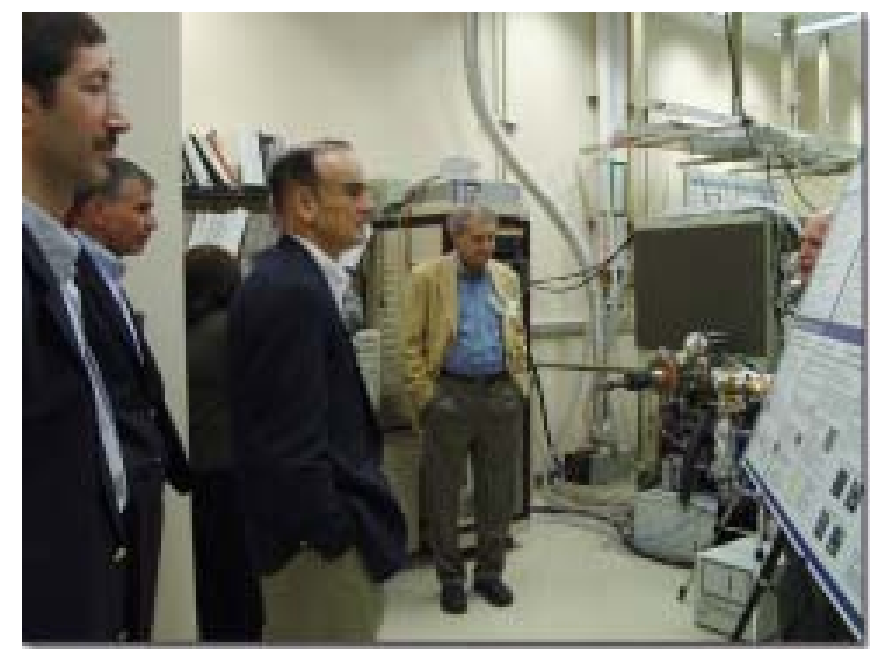

Figure 1.4.1. ESTD's Directorate Review Committee is briefed on state-of-the-art surface analytical capabilities at the Environmental Molecular Sciences Laboratory. 


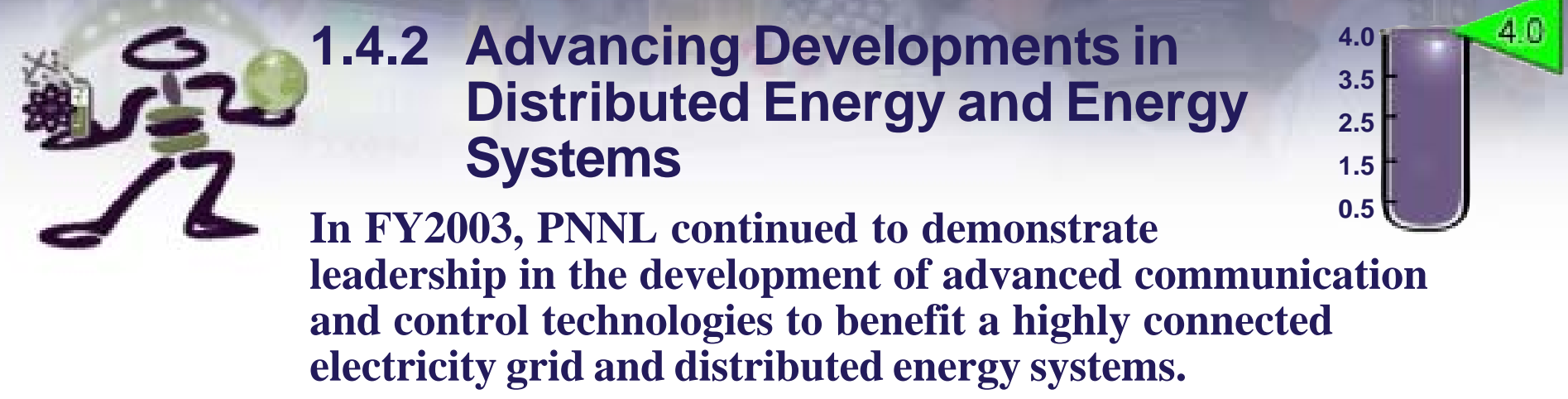

PNNL's technology and leadership are key to transforming the nation's electricity grid into a reliable and efficient national electricity system. Our vision, GridWise ${ }^{\mathrm{TM}}$, is a collaborative network filled with information and a myriad of market-based opportunities, reaching from central generation down to customer appliances and equipment. Key transforming technologies include network-based control systems, distributed intelligence control, autonomous agent systems, energy diagnostics and controls, and building dynamics.

\section{Leadership and Technology Development in Advanced Communications and Controls}

PNNL is developing awareness, relationships, and support for a new national research agenda in Advanced Communications and Controls for transforming the grid. The Laboratory formed the GridWise Alliance Board, a crosscut of industry executives, to guide and review the general direction of GridWise. We have expanded our industry relationships by establishing the GridWise Alliance Industry Consortium to characterize and communicate the societal benefits of infrastructure transformation, collaborate to develop mutually beneficial commercial opportunities, and lobby for needed technical and regulatory changes. In FY2003, we developed a mission statement, summarized stakeholder feedback, and developed materials for dissemination to industry.

October 24-25, 2002, we held a Communication \& Control Systems Distributed Energy Conference, convening over 70 participants from electric utilities, distributed energy equipment manufacturers, information technology providers, state energy agencies, universities, and national laboratories to present the latest developments in communication and control systems for distributed energy.

\section{Electric Distribution Transformation}

PNNL supports the DOE Office of Electric Transmission and Distribution's program to develop a communications and controls environment that will enable transformational change by creating and analyzing grid management tools and by conducting research on demand response as a member of the Consortium for Electric Reliability Technology Solutions.

Following the August 14, 2003, East Coast blackout, PNNL helped analyze data from affected utilities. We also responded to a request from the North American Electric Reliability Council for one of our senior energy systems researcher, Jeff Dagle, to investigate the cause of the blackout.

\section{Energy Systems Transformation Initiative}

PNNL's Energy System Transformation Initiative is investing in the basic science and technology needed to shape and drive this transformation for the benefit of ratepayers and industry. Our research this year, principally in combined grid/markets simulations and in analysis techniques for complex adaptive systems, has yielded insights into potential impacts of price- responsive load in the energy distribution system. We also created a mathematically well-defined computational framework to model and simulate complex economics-driven energy transmission and distribution systems networks.

We also made two excellent technical hires in electric power engineering, averting a staff shortage that could limit our ability to expand GridWise. Both are highly skilled in simulation and analysis, central to our efforts to invent the grid of the future. 


\section{Buildings Technologies, Energy Management, and Weatherization}

As a strategic partner in building technologies, energy management, and weatherization, PNNL continues helping our nation enjoy secure, clean, affordable energy. PNNL's technical contributions and leadership in support of Building Energy Codes Project (BECP) included refinement and supporting analysis of the Residential IECC Code Change (RICC) proposal submitted to the International Code Council (ICC) in early 2003 for the 2004 code change cycle. PNNL successfully worked with DOE to develop support for this comprehensive code change proposal, which has been well received across the building industry, states, and energy efficiency advocacy organizations. PNNL continues to help DOE prepare supporting documentation for the Federal Residential and Federal Commercial Notice of Proposed Rulemaking.

PNNL achieved significant progress within the Emerging Technologies Project, designed to help develop and bring to market advanced energy-efficient products for residential and commercial buildings. Progress included

- Starting Phase III of the efficient recessed downlights project,

- Completing the first phase of the Lighting for Tomorrow residential lighting design competition, and

- Signing agreements with Global Energy and the U.S. Army to begin demonstrating Global Energy's newto-the-market commercial unitary air conditioner at Fort Gordon, Georgia.

To a diverse suite of Rebuild America partners, PNNL provided substantial high-quality technical assistance and training, including installing the Whole Building Diagnostician at the University of Wisconsin; 12 field studies; and three training workshops. In total, across 12 states, PNNL served two universities, four schools, two hospitals, five local governments, and one state government.

\section{Strengthening Regional Relationships}

We have expanded our regional relationships by helping to create the Northwest Energy Technology Collaborative (NWETC), a joint effort of business, government, nonprofit, industry, and educational institutions in the Pacific Northwest to position this as a recognized leader for innovative research, education, and product development for global energy technology markets.

We are developing new relationships, and expanding existing ones, with Bonneville Power Administration, the Northwest Power Planning Council, the Northwest Energy Efficiency Alliance, and public and private utilities.

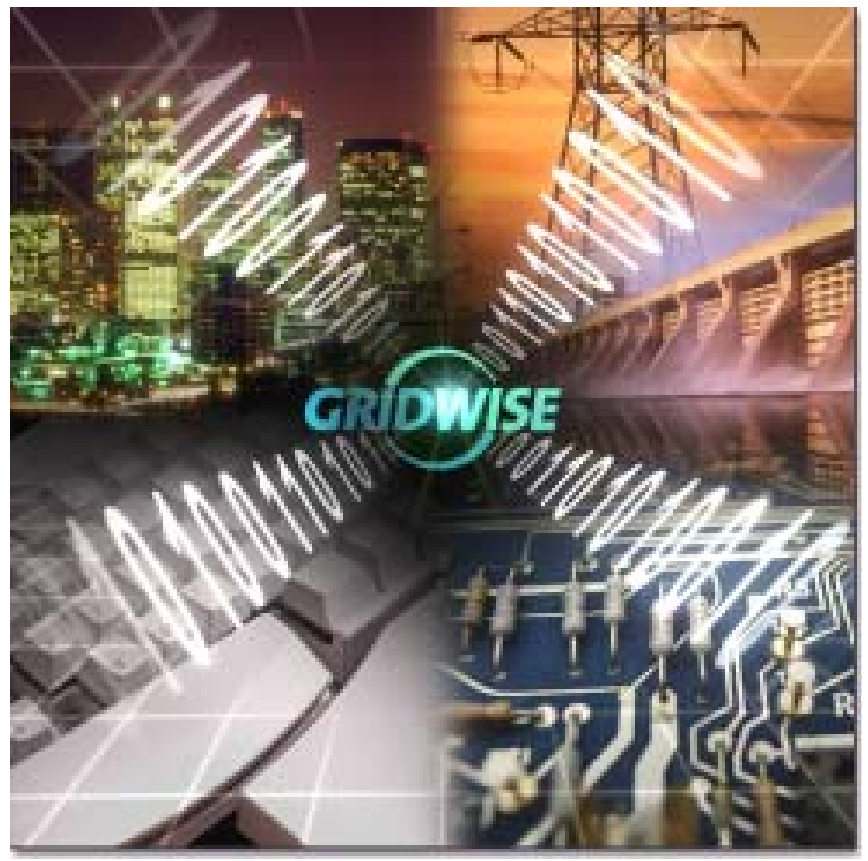

Figure 1.4.2. GridWise, a PNNL-inspired alliance of industry partners bringing energy systems into the modern age with real-time information, e-business systems, and market efficiencies, will minimize the need for inventory and infrastructure and maximize productivity and efficiency. 


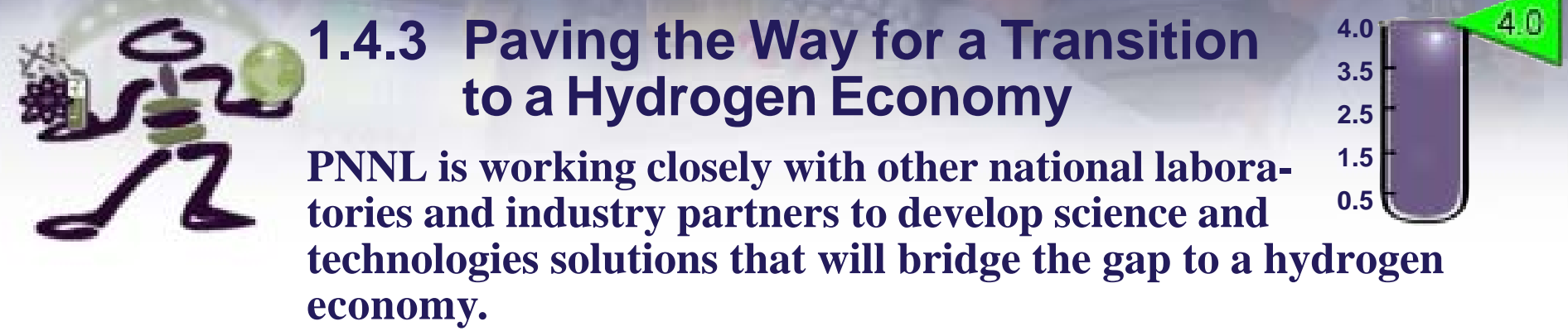

PNNL is committed to the mission and goals of the Hydrogen, Fuel Cells, and Infrastructure Program and currently supports efforts in on-board fuel reformation, fuel cell powered auxiliary power units, and hydrogen safety. Our technical accomplishments in FY2003 were outstanding; we

- Established with DOE-HQ a national hydrogen safety education and training center

- Developed models for fuel cell based essential power systems

- Developed microchannel components for fuel processing

- Performed joint evaluation and testing of microchannel components with industry.

\section{Developing the National Hydrogen Safety Program}

PNNL manages the National Hydrogen Safety Program for DOE's Office of Hydrogen, Fuel Cells and Infrastructure Technologies. PNNL helped DOE review and enhance the hydrogen safety portion of the Multi-Year Program Plan (MYPP), and is working with the DOE management team to define and develop the hydrogen safety program for FY2004 and beyond. PNNL is assembling the Hydrogen Safety Panel described in the MYPP and expected to be in place by November 2003. PNNL is also working with DOE to establish a national hydrogen safety education and training center at DOE's HAMMER facility in Richland, Washington.

\section{Fuel Cell Auxiliary Power Units for Heavy Trucks}

PNNL is leveraging the laboratory's signature capabilities to support the Hydrogen Fuel Initiative. In FY2003, PNNL continued close collaboration with the headquarters Hydrogen Program, the National Energy Technology Laboratory (NETL), and industry (PACCAR, Caterpillar, and Delphi Automotive Systems) to define a technical program in Solid Oxide Fuel Cell (SOFC)-based essential power systems (EPS) for heavy trucks. PNNL technical efforts during FY2003 focused on providing modeling tools for understanding auxiliary power units (APU) system and long-term performance through two technical activities:

- Developing a control system for both heat-up and operating phases of the APU. A control system would improve fuel efficiency and operating lifespan in APU systems.

- Developing models and experiments to examine mechanical durability of the stack in APUs. The results from this activity will be used to design SOFC stacks, cell, and sealing configurations that are durable during transportation.

\section{Collaborating to Develop Hydrogen Storage Technologies}

In September 2003, PNNL and Los Alamos submitted a proposal to become one of six hydrogen storage centers, focusing on chemical hydrides for storage. This approach builds on PNNL's materials and strong catalysis capabilities. We also continued exploring options for generating hydrogen from renewable resources, pursuing options such as high-temperature steam electrolysis, gasification of biomass feedstocks, and photo-catalyzed water splitting using single-cell algae.

\section{Microchannel Technologies Support Goals of the FreedomCAR Program}

Novel fuel reformer technologies are being developed to support the goals of DOE's FreedomCAR Program, including

- an autothermal fuel processor that can meet fast startup requirements for on-board applications, and a microchannel mixer to uniformly mix air into the reformate during start-up, 
- a microchannel steam reforming subsystem that can achieve cold start in 30 seconds or less, consistent with 2010 FreedomCAR performance targets for onboard fuel reformers; incorporates significant improvements in reactor productivity and sulfur tolerance,

- a differential temperature water gas shift reactor (which produces hydrogen by reacting carbon monoxide with water) that is two to three times more compact than can be achieved using conventional, twostage adiabatic designs, and

- a preferential oxidation reactor (PROX) subsystem that incorporates microchannel technology to improve temperature control and reduce the number of required stages. With multiple air inlets, the microchannel PROX subsystem provides the best combination of high carbon monoxide conversion, low hydrogen combustion, and low reverse water gas shift involving carbon dioxide.

PNNL also sent microchannel components to industrial developers for testing and evaluation. For example, steam generators sufficient for a 50 kilowatt-electric autothermal fuel reforming system went to McDermott and to the Gas Technology Institute; heat exchangers and vaporizers went to Innovatek for a fuel processing system being developed for the U.S. Army; and a microchannel economizer currently is being developed for Cleaver-Brooks and the Gas Technology Institute for use in a highefficiency boiler system.

\section{Building Regional Awareness and Relationships for DOE's Hydrogen Program}

We have built regional awareness and are promoting user acceptance of hydrogen and fuel cells in support of a future hydrogen economy. PNNL hosted the Hydrogen Production and Northwest Transportation Conference in Seattle June 2003. Approximately 150 people from industry, government, and business gathered to hear how the Northwest could play a leading role in moving toward a hydrogen economy. The conference included appearances by PNNL Laboratory Director Len Peters; Washington State Governor Gary Locke; and Steve Chalk, who leads the Department of Energy's Hydrogen, Fuel Cells and Infrastructure Program. It also featured presentations by technology companies, the Bonneville Power Administration, DOE, and DoD.

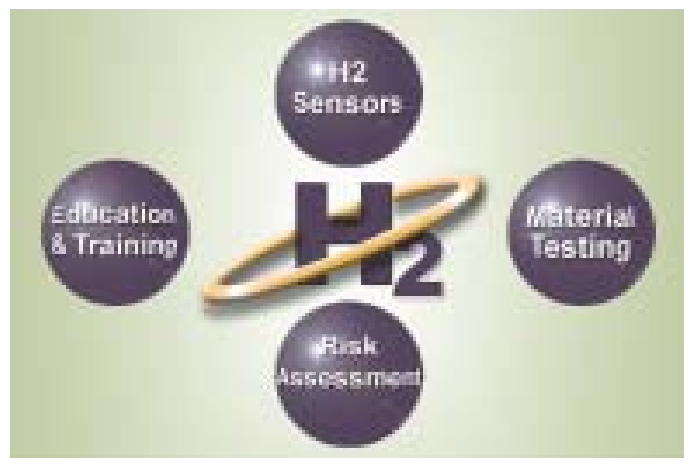

Figure 1.4.3. PNNL is leading the DOE Hydrogen Safety Program and coordinating partnerships to bring safe practices and operations to all facets of the DOE hydrogen program. 


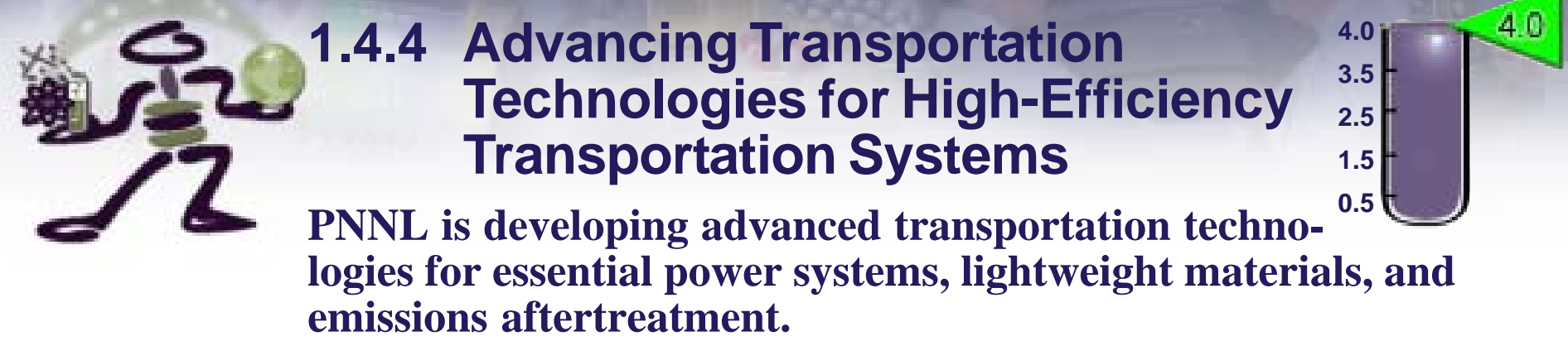

PNNL partnerships promote the development and use of advanced transportation vehicles and alternate fuel technologies. In FY2003, PNNL demonstrated both outstanding technical contributions to, and support of, existing and new programmatic areas in the Office of Freedom Car and Vehicle Technologies (OFCVT). For these contributions, PNNL received a Federal Laboratory Consortium Award for excellence in technology transfer for the developing an engine exhaust aftertreatment system based on non-thermal plasma-assisted catalysis and for transferring this technology to Ford, General Motors, DaimlerChrysler, Caterpillar, and Delphi.

\section{New Lightweight Materials for Automotive and Heavy Vehicle Applications}

PNNL's technical contributions include developing new lightweight materials and manufacturing technologies that enable their increased use for automotive and heavy vehicle applications. Specific accomplishments in FY2003 included

- Developing and testing a unique new aluminum metal matrix composite brake rotor in partnership with USCAR and Visteon Automotive. With potential for production, the lightweight brake rotors could save $20 \mathrm{Kg}$. per vehicle;

- Completing important project efforts focusing on developing thermoplastic composite materials forming techniques, investigating hydroforming of aluminum, and developing lightweight glazing for automotive applications;

- Collaborating with ORNL to start commercial heavy truck projects on composite joining, carbon fiber molding compounds, and low-cost rapid tooling technology to reduce weight and benefit manufacturing;

- Providing continued planning and strategic support to the automotive lightweight materials and High Strength/ Weight Reduction Materials program; and
- Drafting the Fuel Cell Vehicle Materials research roadmap for planning with government and industry stakeholders.

\section{Applying PNNL Catalysis Capabilities to Reduce Diesel Emissions}

PNNL continues to develop advanced after-treatment technologies to reduce diesel emissions, an essential step toward the adoption of highly efficient diesel engines for trucks and passenger cars. In FY2003, our accomplishments in this area include

- A successful engine test of plasma catalysis technology that reduced $\mathrm{NO}_{\mathrm{x}}$ by as much as $70 \%$ using diesel fuel as the reductant, carried out by the plasma catalysis for light-duty diesel vehicles program;

- Producing, as leader of a cooperative program with Caterpillar, a new class of materials that have surpassed all initial project targets for sulfur capacity;

- Playing an instrumental role in forming a collaborative effort with Sandia and Oak Ridge in the area of diesel emissions modeling. Within this program PNNL leads the sub team activities in diesel particulate filtration;

- In cooperation with Delphi Corporation, identifying a Pt alloy electrocatalyst for $\mathrm{NO}_{\mathrm{x}}$ sensors that provides an approximate 10-fold improvement in activity while maintaining high selectivity; and

- Starting a large research program with GE to develop aftertreatment systems for future diesel locomotives.

\section{Thermoelectric Devices}

In a program aimed at emerging technology to recover waste heat in internal combustion engines, PNNL has fabricated quantum thin film structures required for efficient thermoelectric devices and demonstrated the promising electrical properties of multilayer thin films. 
Supporting the DOE $21^{\text {st }}$-Century Truck Initiative

PNNL helped launch the DOE $21^{\text {st }}$-Century Truck Initiative, including leading the development of the technical needs and roadmap. We also helped establish a laboratory coordinating council for $21^{\text {st }}$-Century Truck Initiative and chair the council. PNNL staff consistently contributed to the OFCVT mission by participation in the Technical Roadmap, the OFCVT annual offsite workshop, and the Diesel Engine Emission Reduction Workshop. PNNL continues to contribute to the Multilaboratory After-treatment Program, the annual CIDI Review, FreedomCAR Material program and the

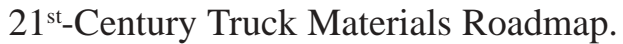

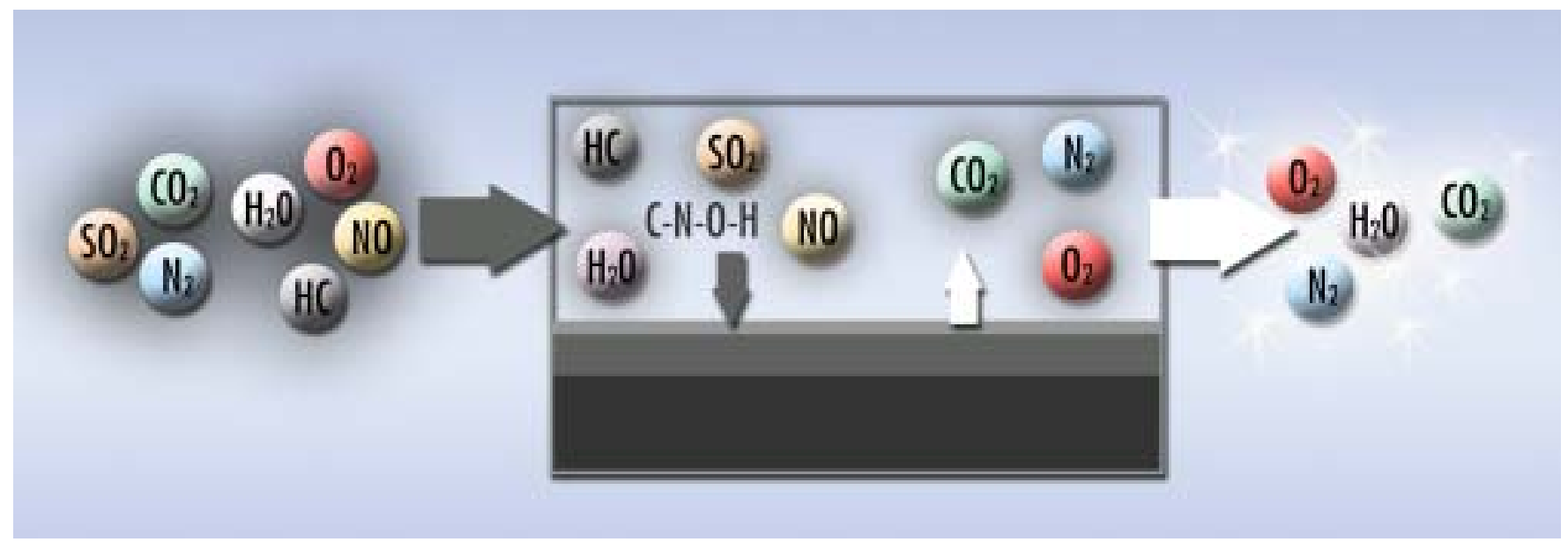

Figure 1.4.4. PNNL applies its expertise in surface chemistry and catalysis to develop technologies that reduce harmful emissions from diesel engine exhaust, converting oxides of nitrogen and particulate matter into components of clean air. 


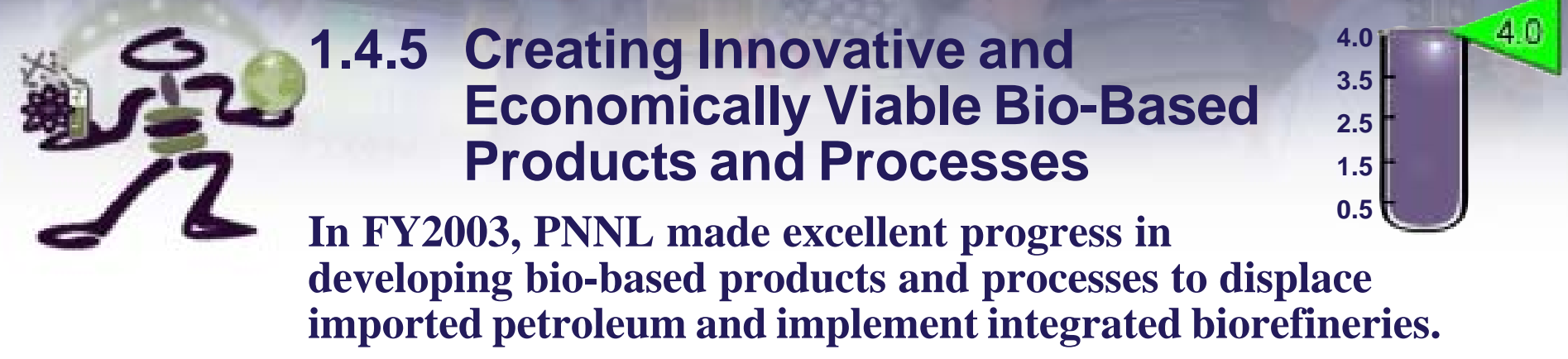

The major goals of the Office of the Biomass Program (OBP) are to reduce dependence on foreign oil and establish integrated biorefineries that produce a costeffective combination of products, fuels, and power. In FY2003, PNNL continued innovative, high-quality research, and worked effectively with industry to help DOE create economically viable biomass technologies.

In FY2003, \$1.5 million in OBP capital funds underwrote PNNL's new distinctive signature capability for combinatorial catalysis instrumentation, which allows rapid formulation, screening, and analysis of advanced catalysts for biomass systems. Known as the "Combicat," it provides a greater range of operational pressures and temperatures than previously available, and has already been used to collect data for ongoing projects. PNNL helped design this unique instrumentation, available nowhere else, and worked with the vendor to deliver the product ahead of schedule and on budget.

\section{DOE's Biomass Program Integration}

During 2003, PNNL worked closely with NREL and other national labs to help OBP achieve its objective of having a single, integrated biomass program. PNNL was a key contributor to OBP's integrated Multi-Year Program Plan and Annual Operating Plan. Establishing a consistent, integrated program with cooperation of major labs through DOE's National Bioenergy Center (NBC) was a key 2003 OBP objective. PNNL's significantly improved relationship with NREL and other NBC labs (ORNL, INEEL, and ANL) has enabled OBP to use the capabilities of individual labs much more effectively while coordinating those efforts for the larger benefit of the program.

PNNL has identified opportunities to involve major agricultural and industry partners in the research. By providing cost sharing, these partners leverage DOE investments, and their involvement ensures the research meets the needs of industry. In FY2003, PNNL conducted projects funded either by OBP or jointly by OBP/USDA totaling approximately $\$ 5.5$ million.

\section{Outreach and Collaborations with Industry and Government}

PNNL expanded its efforts in the area of bio-based products this fiscal year. Bio-based products are important to help OBP meet its goals by creating opportunities for higher economic return within an integrated biorefinery. In 2003, as a result of competitive solicitations led by industry, PNNL was awarded four Cooperative Research and Development Agreement (CRADA) projects. Major partners included Cargill, the National Corn Growers Association/ADM, and the Iowa Corn Promotion Board, which leads two projects in collaboration with Kemin Industries and grain producer associations from Minnesota and Ohio. These projects, which help accelerate commercial implementation of DOE-funded concepts, continue PNNL's excellent working relationship with leading agricultural companies.

In addition, PNNL led a project funded directly by DOE to identify the top bio-based intermediates from 5- and 6-carbon sugars. This project, in collaboration with NREL, is expected to lead to the identification of the most promising opportunities for bio-based products research.

Industry recognizes the value of our research to gasify wet biomass, to convert biomass syngas to liquid fuel at scales commensurate with the biomass resource, and to examine the opportunities for biomass pyrolysis as a source of liquid biofuels. In FY2003, PNNL continued RD\&D activities in support of OPB's Syngas Platform in all these areas. An August 2003 industry-led review found this work innovative.

\section{Strong Regional Collaborations}

PNNL continued efforts to use the capabilities of regional entities to help establish a foundation for an 
expanded bioproducts industry. In FY2003, PNNL worked with Washington State University (WSU) to establish a new research building on the WSU campus. As a result of this partnership, the State of Washington is providing funding to conduct detailed engineering and design studies for a bio-based products research facility. The facility is expected to result in both a more efficient research facility in which to conduct the DOE research (without the need for a specific DOE building appropriation) and a more effective working relationship with WSU.

\section{Creating Intellectual Property for DOE and PNNL}

As another sign of the significance of PNNL's programs, innovation, and technical achievements in developing value added bio-based products and processes, we received three patents and submitted six new patent applications in FY2003. The development of this type of intellectual property is vital to industry and provides a basis for investment in new bio-based technologies for commercial deployment.

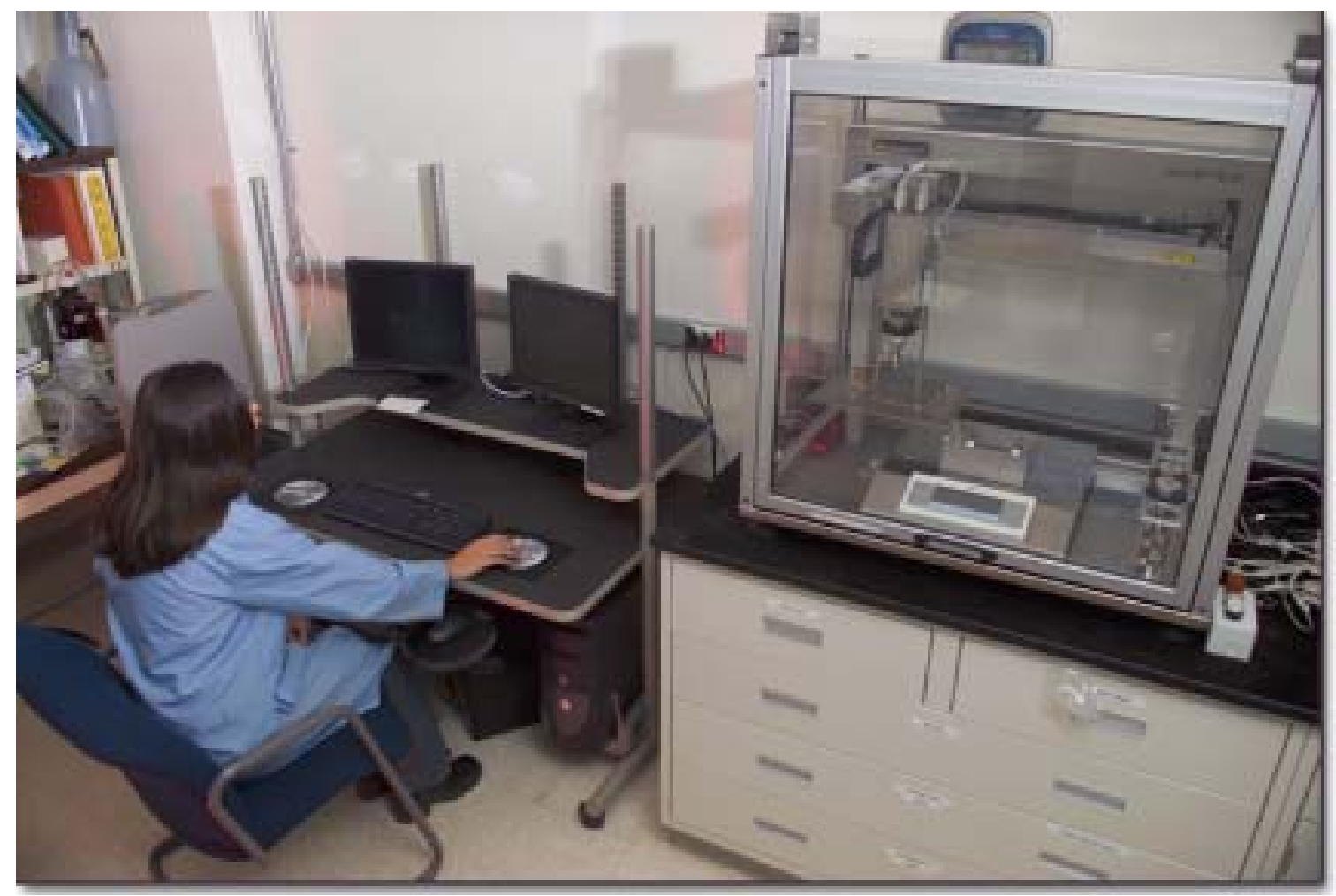

Figure 1.4.5. The new state-of-the-art "Combicat" instrumentation allows the rapid formulation, screening, and analysis of advanced catalysts for biomass systems. 


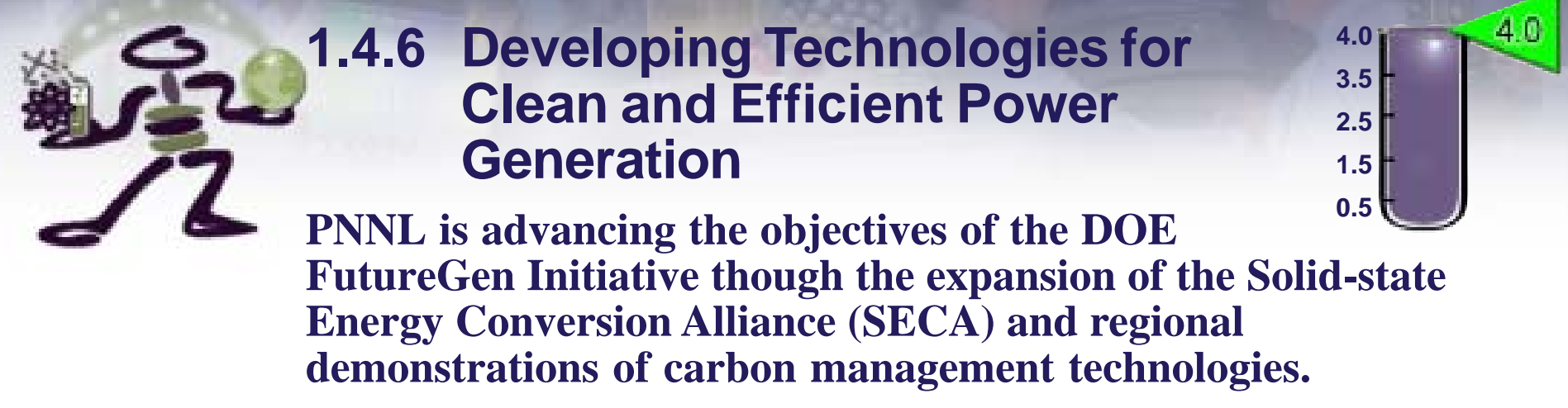

PNNL's R\&D supports DOE's Office of Fossil Energy's (FE) efforts related to fuel cells, hydrogen and other clean fuels, and carbon sequestration, all of which align with the President's recently announced FutureGen Initiative. DOE calls this FE-led Initiative its "highest priority within the coal research effort." FutureGen will involve designing, building, and operating the world's first near zeroemissions coal-fueled power plant. If it fully materializes, this project will be one of the most significant accomplishments across the DOE energy program offices in decades.

In FY2003, PNNL provided outstanding leadership and technical contributions, with accomplishments including

- Expanding the Solid State Energy Conversion Alliance;

- Conducting joint research as part of the High Temperature Electrochemistry Center (HiTEC);

- Helping establish the Center for Zero-Emissions Research and Technology;

- Pursuing Carbon Management and Carbon Sequestration projects, including participation in one regional partnership; and

- Participating in and providing technical leadership to the FutureGen Industrial Alliance.

\section{Continued Leadership in the SECA Program}

SECA's mission is to accelerate the commercialization of low-cost solid oxide fuel cells (SOFC) as quickly as possible over the next decade. Under the leadership of the National Energy Technology Laboratory (NETL) and PNNL, and with support from FE, this alliance of U.S. industry, universities, and other research organizations is striving to make the breakthroughs required to mass produce a modular form of SOFC for $\$ 400$ per kilowatt. In FY2003, two new industrial teams, Acumentrics and Fuel Cell Energy, joined the program.
In April 2003, PNNL coordinated the Fourth Annual SECA Workshop, which attracted nearly 200 participants to Seattle. Focusing on fuel cell applications beyond central power, the Workshop also, and for the first time, included displays of fuel cells that demonstrated the progress of the SECA industrial teams.

In FY2003, the SECACore Technology Program's (CTP) numerous technical accomplishments included

- Lanthanum ferrite-based cathode materials offering improved electrical performance in intermediate temperature cells (e.g., $700-800^{\circ} \mathrm{C}$ );

- Improved ceramic composite anode materials offering redox, sulfur, and carbon tolerance;

- Mica-based compressive seals offering low leak rates and improved thermal cycle stability (compared to conventional glass-ceramic seals);

- Comprehensive evaluation of state-of-the-art interconnects alloys;

- Computational thermal, stress, and electrochemical models for better understanding of fuel cell processes and the optimization of the cell and stack designs for high performance and reliability during thermal cycling;

- Active participation in university and industry outreach activities including invited presentations to NASA Glenn Research Center, Siemens Westinghouse Electric Corporation, and General Electric Power Systems;

- Coordinating a multi-agency, industry-led SOFC seal development workshop at Sandia National Laboratories; and

- Conducting an industry-wide training program on advanced computational tools developed under the SECA-CTP.

\section{HiTEC Highlights}

This collaborative research program, established in FY2002 to make advancements in electrochemistry for 
next-generation energy conversion systems, will address the underpinning technical issues associated with FutureGen. PNNL and NETL coordinate HiTEC's activities, including a hub at PNNL and the first satellite center at Montana State University. MSU and PNNL researchers focused on separations technology for advanced power systems, emphasizing high-temperature applications, $\mathrm{CO}_{2}$ capture, and hydrogen separation from coal gas.

\section{HiTEC R\&D Highlights in FY2003 Include}

- Development of new anode materials for SOFCs that enable fuel cells to operate at lower temperatures and in environments with higher sulfur contents, and

- Identifying degradation mechanisms for embedded interfaces within an operating fuel cell, making it possible to extend the lifetime of fuel cells.

\section{Coordinating DOE Programs to Develop Essential Power Systems}

As part of a joint project for $\mathrm{FE}$ and the Office of Energy Efficiency and Renewable Energy (EERE), PNNL is working with the Technology Development and Program managers for EERE's Hydrogen Program, NETL, and industry to define a technical program on SOFC-based essential power systems (EPS) for heavy trucks. Research results will be used to design transportationworthy SOFC stack, cell, and sealing configurations.

\section{SECA Fuel Cell Operated on Gasified Coal}

A key FY2003 accomplishment was the successful demonstration in June of a SECA fuel cell operated on gasified coal. Delphi Automotive Systems, one of SECA's industrial teams, provided the stack for the test, which took place at the Power Systems Development Facility coal-gasification plant managed by Southern Company in Wilsonville, Alabama. This success is a major step on the path to FutureGen.

\section{Center for Zero Emissions Research and Technology (ZERT):}

In FY2003, PNNL helped create the Center for Zero Emissions Technology, which will be established at and coordinated by Montana State University in FY2004. Including PNNL and NETL, this center will focus on geologic sequestration and advanced fossil-fueled power systems integration.

\section{Regional Carbon Partnerships (RCP)}

PNNL provided the technical leadership, and Battelle Columbus provided the management leadership, that lead to the MidWest Regional Carbon Partnership (RCP), announced by the Secretary of Energy in late August. One of nine national partnerships, the MidWest RCP is expected to be among the most important, as it covers West Virginia, Ohio, Pennsylvania, Kentucky and Indiana - the heartland of U.S. coal country. This partnership includes the states' geologic surveys and major universities.

\section{Partnering with Industry to Develop the FutureGen facility}

PNNL, Battelle, and ten major corporations have formed the FutureGen Industrial Alliance to design, build, and operate the FutureGen facility. Alliance charter member companies represent more than 45 percent of U.S. coal production and more than 20 percent of U.S. coal-fueled power production. Along with industry and NETL, PNNL aspires to be a major technical contributor to this highprofile effort and intends to provide significant intellectual leadership during conceptual design.

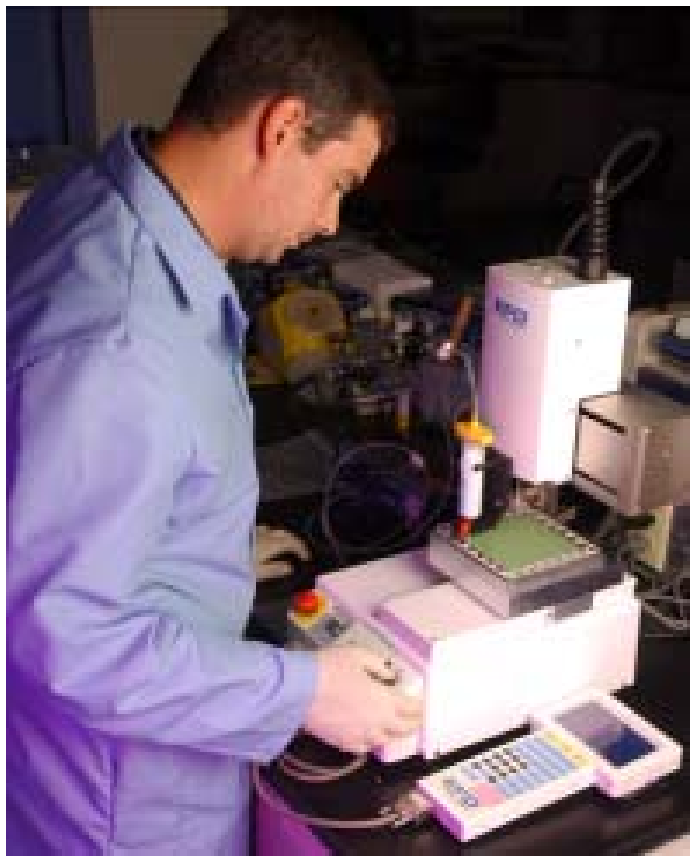

Figure 1.4.6. PNNL researchers use robotically dispensed seals for improved seal strength for solid oxide fuel cell stacks. 


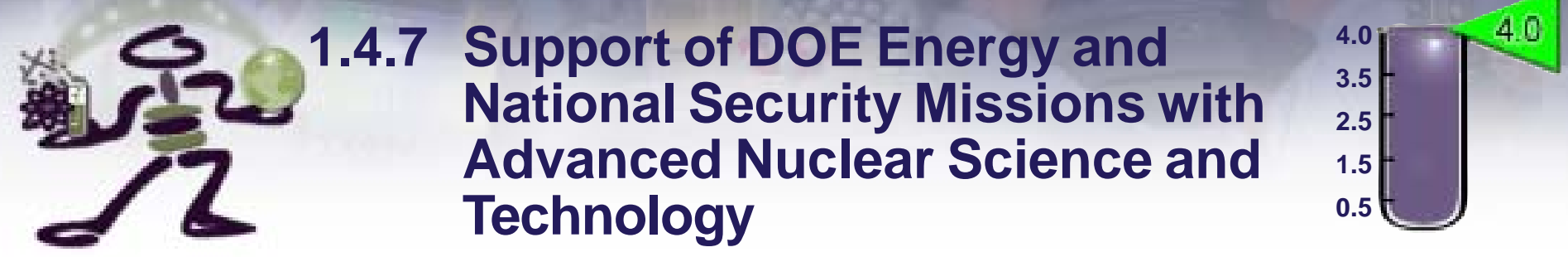

\section{PNNL is sustaining and building national capabilities in nuclear science and engineering in support of the nuclear component of national energy and security goals.}

Our nuclear energy, science, and technology resources include unique capabilities in advanced materials, advanced separation methods, advanced techniques for non-destructively detecting and analyzing flaws in safetyrelated reactor components, advanced instrumentation and controls for next-generation reactors, and related capabilities necessary for securing a safe and viable nuclear energy option.

\section{Science \& Technology to Support DOE Nuclear Energy}

PNNL continues to provide effective leadership in our role as Executive Agent for the Office of Nuclear Energy, Science and Technology's (DOE-NE) International Nuclear Energy Research Initiative (I-NERI). In December 2002, five awards were made for U.S.I Republic of Korea collaborative projects in advanced instrumentation, controls and diagnostics; advanced light water reactor (LWR) technology; advanced LWR fuels and materials technology; LWR safety technology; and advanced LWR computational methods. In March 2003, a new project, part of the U.S./France collaboration, began, increasing the number of projects with France to five. The Laboratory supported DOE-NE in establishing an agreement to collaborate on nuclear-related technology research and development with the European Union, and bilateral agreements with Canada and Brazil. In September, PNNL helped NE prepare for annual program reviews with France and Korea.

Also in FY2003, the NASA launched Project Prometheus (formerly the Nuclear Systems Initiative) to develop nuclear-powered rockets and on-board nuclear generators for extended solar system exploration. PNNL is supporting an important component of Project Prometheus - the Jupiter Icy Moons Orbiter (JIMO) - through a contract with DOE-NE-50 (Office of Space and Defense Power Systems). Funded by NASA via NE-50, the Laboratory supports post-irradiation exami- nation of fuel pins from the earlier SP-100 space power program, ground nuclear test costing and lessons learned, design of annular linear induction pumps, and safety.

At the request of DOE-NE, Dr. Alan Waltar, PNNL's Nuclear Energy Director, is chairing an international "Blue Ribbon Committee" to advise Nobel Laureate Dr. Burton Richter and his Nuclear Energy Research Advisory Committee (NERAC) subcommittee on alternative approaches for closing the commercial nuclear fuel cycle without compromising the nation's non-proliferation and national security objectives.

\section{PNNL's Integrated Nuclear Strategy (INS)}

PNNL completed its Integrated Nuclear Strategy in March 2003. This report documents a systematic, labwide evaluation of the current status and future prospects of the nuclear component of the Lab's multi-program portfolio. The Strategy focuses on three areas: 1) Legacy (DOE and international cleanup efforts);

2) Nuclear, biological, and chemical threats; and

3) Energy (commercial nuclear power via current-generation as well as future-generation technologies). Key conclusions note that

- PNNL's nuclear program and capabilities are vital to PNNL's ongoing role as a multi-program national laboratory;

- The need to counter terrorist threats of nuclear, biological, and chemical weapons proliferation and use will have substantial demands for PNNL capabilities; and

- PNNL has well-recognized R\&D capabilities in understanding irradiation effects on reactor structural materials, in composite materials for advanced reactors, and in non-destructive techniques for identifying and evaluating flaws in structural components. 
PNNL Provides New and Sustainable Radiological/Nuclear Capabilities and Programs through the Radiation Detection and Analysis Center

In FY2003, PNNL refocused the Advanced Nuclear Science and Technology Initiative (ANSTI) to align with the Integrated Nuclear Strategy. In particular, ANSTI developed the framework for establishing a Radiological Detection and Analysis Center (RDAC) in FY2004 to tailor these capabilities for national security and homeland defense applications.

In addition, we made substantial progress in FY2003 on developing new and enhanced nuclear science and technology capabilities, particularly in the nonproliferation and homeland security areas. The Advanced /Automatable Radiochemical Analysis project, for example, will lead to advanced analytical methods for characterizing radionuclides. PNNL also submitted a joint proposal to DOE NA-22 (Office of Nonproliferation Research and Engineering) with several university collaborators aimed at developing the next generation of graduate-level radiochemists through joint R\&D projects and associated mentoring and guidance.

\section{PNNL is fulfilling DOE's commitment to the Nuclear Regulatory Commission (NRC) in support of the NRC safety mission.}

Reactor vessel integrity and cracking/corrosion-related damage are significant safety/lifetime issues for the NRC. The Laboratory continues to provide internationally recognized expertise in non-destructive examination (NDE) techniques to verify the safety margins for large steel components and steam generator tubes. In FY2003, PNNL staff with NDE expertise assisted in evaluating techniques used to determine the origin of the reactor vessel leaks at the South Texas Nuclear Project. PNNL staff also evaluated corrosion damage in the reactor vessel head of the Davis-Besse Nuclear Power Station.

PNNL continues to provide significant support to the NRC license renewal process by evaluating the environmental impacts of requests for 20 -year extensions. In FY2004, the Laboratory anticipates expanding its role in this area to include reviewing essentially all safety aspects of license renewal.

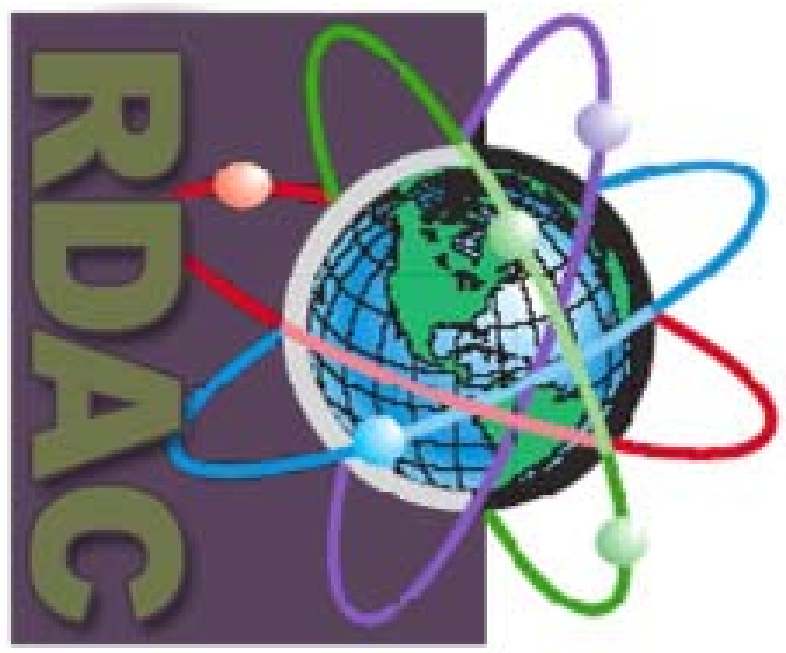

Figure 1.4.7. The new Radiation Detection and Analysis Center (RDAC) will consolidate the Laboratory's wide range of radiation detection and instrumentation, simulation and analysis, and nuclear fuel cycle expertise into one integrated effort focused on national defense and homeland security. 



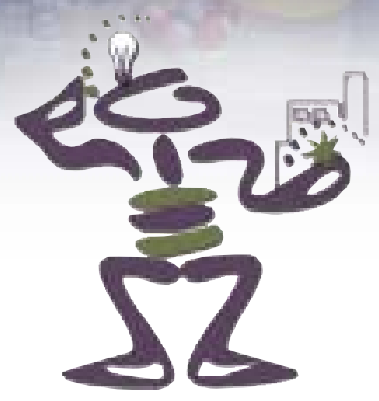

Part I

\section{Status of Performance Against Critical Outcomes 2.0 Management and Operational Excellence}

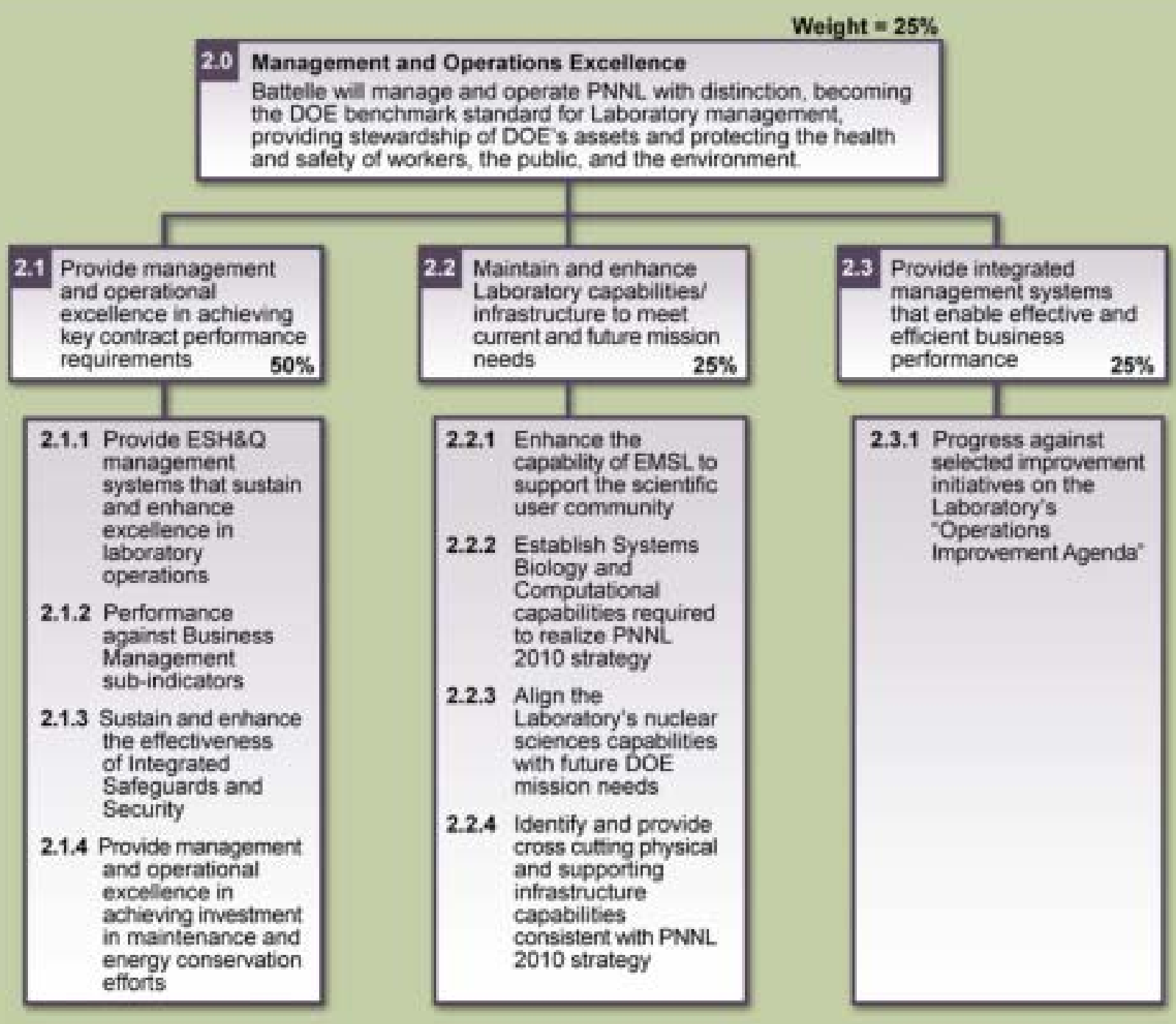


$\begin{array}{ll}2.0 & \text { Status of Performance Against } \\ \text { Critical Outcome 2.0: Management } & \text { and Operations Excellence }\end{array}$

Battelle managed and operated the Laboratory with distinction, becoming the DOE benchmark for Laboratory management, providing stewardship of DOE's assets, and protecting the health and safety of workers, the public, and the environment. In FY2003, PNNL met or exceeded nearly all DOE expectations in Management and Operations Excellence.
This section of the report details PNNL's self-evaluation of the adjectives and corresponding value points that lead to PNNL's Critical Outcome 2.0 FY2003 point total of 3.88 and performance rating of Outstanding for Management and Operations Excellence. Appendix A shows the adjectival and value ratings for FY2003 Critical Outcome 2.0 and all of its objectives and performance indicators (Tables A.8 - A.14).

\section{Highlights of the Three Performance Objectives}

\subsection{Provide Management and Operations Excellence in Achieving Key Contract Performance Requirements}

$\checkmark$ 2.1.1 Goal: Meet targets for 7 or more of 8 ESH\&Q measures. Result: PNNL met or exceeded targets for all 8 measures.

$\checkmark$ 2.1.2.1 Goal: Achieve overhead costs of $51 \%$ or less of the total 1830 average charge out rate. Result: PNNL achieved an overhead cost of $50.4 \%$.

$凶$ 2.1.2.2 Goal: Improve labor cost multiplier by $2 \%$ or more over FY2002. Result: PNNL improved labor cost multiplier by $1 \%$.

囚 2.1.2.3 Goal: Achieve FY2003 direct FTEs greater than or equal to $51 \%$ of total Laboratory FTE's. Result: PNNL achieved a direct FTE rate of 50.4\%.

$\checkmark$ 2.1.3 Goal: Achieve a composite score of $3.5-4.0$ for 11 ISSM indicators. Result: PNNL achieved a composite score of 3.6.

$\checkmark$ 2.1.4.1 Goal: Achieve a stewardship index of .98 or better. Result: PNNL achieved a stewardship index of 1.0 . $\checkmark$ 2.1.4.2 Goal: Meet at least 6 of 7 energy conservation milestones. Result: PNNL met all 7 milestones.

2.2 Maintain and Enhance Laboratory Capabilities/Infrastructure to Meet Current and Future Mission Needs

$\checkmark$ 2.2.1.1 Goal: Meet all six $900 \mathrm{MHz}$ magnet milestones. Result: PNNL accomplished 6 of 6 actions.

$\checkmark$ 2.2.1.2 Goal: Bring Phase 1 and Phase $2 \mathrm{HP}$ Supercomputer to full operational status. Result: PNNL met the goal.

$\checkmark$ 2.2.2.1 Goal: Establish an operating pilot proteomics facility in LSL II and characterize 4 or more complexes. Result: PNNL established the facility and characterized 5 complexes.

$凶$ 2.2.2.2 Goal: Complete all three deliverables critical to meeting computational sciences needs across major PNNL research areas. Result: PNNL completed (1) the requirements assessment and (2) procurement but decided to postpone (3) hiring a Division Director.

$\checkmark$ 2.2.3.1 Goal: Complete all three Integrated Nuclear Strategy milestones and achieve Management Council decision on path forward. Result: PNNL met this goal.

$\checkmark$ 2.2.3.2 Goal: Establish 4 or more joint nuclear R\&D projects with academia, or create 4 or more student positions. Result: PNNL met the goal by creating at least 22 new student positions; the stage is set for $\mathrm{R} \& \mathrm{D}$ collaborations.

$\checkmark$ 2.2.4.1 Goal: Meet at least 5 of 6 internet connection technology infrastructure milestones. Result: PNNL met 5 milestones. 
2.2.4.2 Goal: Meet at least 4 of 5 classified computing infrastructure milestones. Result: PNNL met 4 of 5 milestones.

$\checkmark$ 2.2.4.3 Goal: Meet all 5 milestones relative to developing and implementing a Hanford 300 Area strategy. Result: PNNL met all 5 milestones.

\subsection{Provide Integrated Management Systems that Enable Effective and Efficient Business Performance}

$\checkmark$ 2.3.1 Goal: Meet at least 8 of 9 milestones relative to selected improvement initiatives on the Laboratory's "Operations Improvement Agenda." Result: PNNL met all 9 milestones - and more.

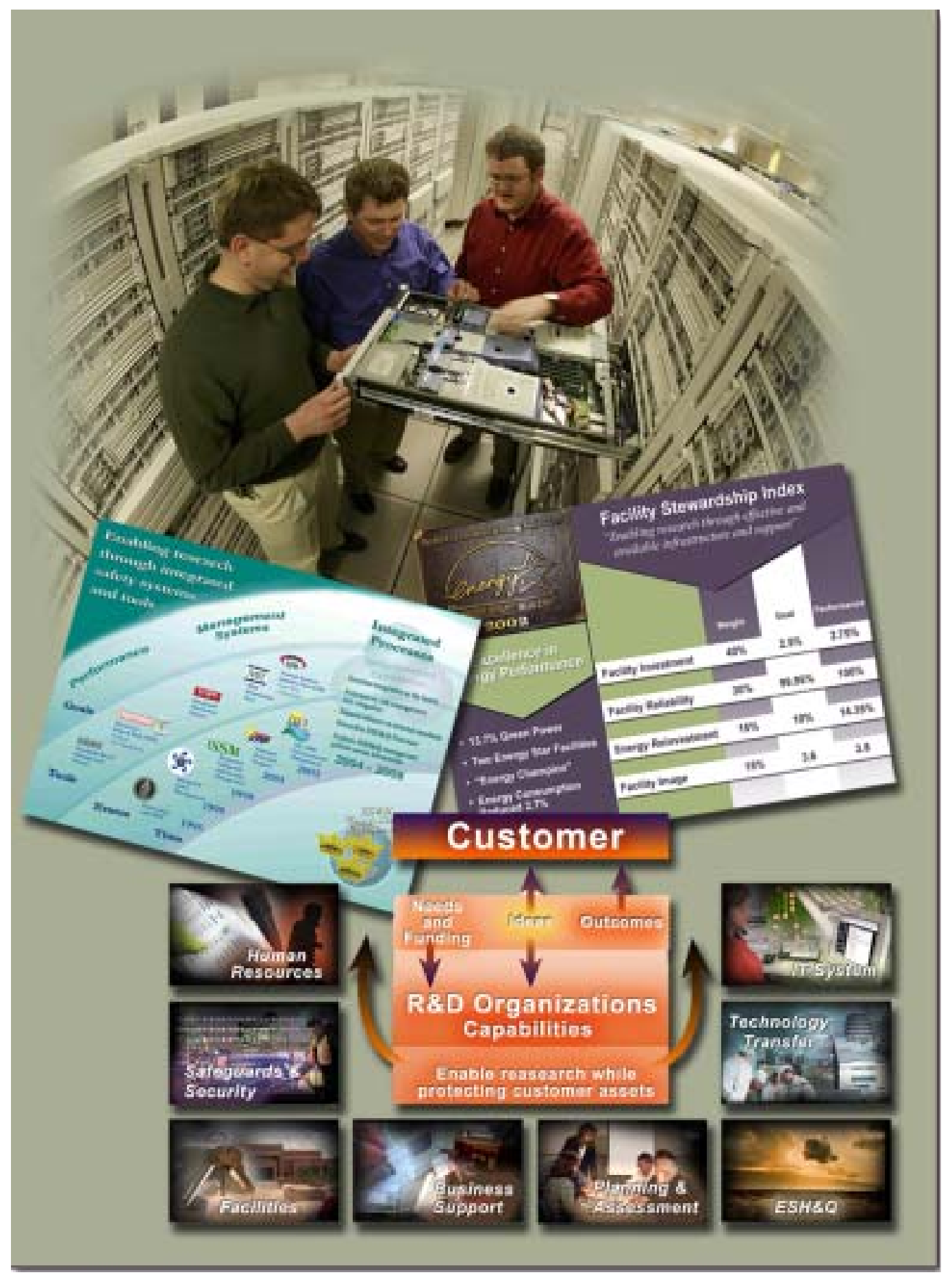

Figure 2.0. PNNL managed and operated the Laboratory with distinction as evidenced by its commitment to facilities and equipment upgrades, environmental safety and health performance, safeguards and security performance, and management system improvements. 


\section{Performance Against Indicators}

All eight measures tracked under 2.1.1 have met or exceeded agreed-upon performance expectations. Table 2.1.1 shows FY 2003 targets and actuals.

We achieved a third straight year of zero environmental releases.

We maintained our ISO14001 environmental management system (EMS) registration. An internal audit conducted under our Independent Oversight group confirmed conformance to the standard and effectiveness of the EMS (see report \#IO-2003-17).

PNNL maintained its Voluntary Protection Program (VPP) STAR status. We improved our FY2002 annual VPP self-evaluation rating from 9.2 to 9.5 . The evaluation results substantiate the overall strength of the Safety $\&$ Health program.

For the third year in a row we have had no spread of radioactive contamination meeting DOE M232.1-1A ORPS reporting Group 1D, "Loss of Control of Radioactive Material/Spread of Radioactive Contamination."

We met our goal to reduce Laboratory-generated hazardous waste. The goal was 11.9 metric tons, the year to date actual was 8.5 metric tons. Figure 2.1.1 shows PNNL's progress over the last 10 years to decrease output.

Two other indicators of the Safety \& Health program are the Total Recordable Case Rate (TRCR) and the Days Away, Restricted or Transferred (DART) case rate. PNNL's performance continues to be good/very good compared to industry rate averages. Table 2.1.1 shows the industry rates (same as the targets) for the TRCR and DART case rates (2.5 industry case rate compared to PNNL's 2.0 for TRCR, and 1.1 industry case rate and 1.0 for PNNL's DART).

\section{Opportunities for Improvement}

Although our ESH\&Q management systems are very good, our self assessment processes indicate there is room for improvement.

\section{High Magnetic Field Safety}

After a hammer was drawn into a superconducting magnet causing an injury to a researcher's finger, PNNL conducted a comprehensive review of our magnet safety practices, and made changes to the engineered barriers and operating practices. After the internal review, PNNL management, deciding that a review by outside experts might provide additional insights, commissioned a review by an external expert and internal subject matter experts in early July. The review team made some recommendations, which we're implementing, but concluded that the overall high magnetic field safety program is in excellent shape. (see Module 1.1.8)

\section{Electrical Shock}

A researcher received an electrical shock of $75 \mathrm{~mA}$ (.075Amps) direct current while working with thin layer coating (sputtering) equipment. At the time, the equipment power supply was operating at 0.5 amps and 500 volts direct current. The staff member could have been injured or killed, but was not; and no apparent negative health effects have been observed. After investigative actions initiated by PNNL management, corrective actions included taking equipment out of service; medically evaluating the employee's condition; and conducting a causal analysis after line management sought an independent review by PNNL's Independent Oversight (IO) organization (Report \#IO-2003-13). Similar highrisk electrical equipment was reviewed by subject matter experts, a lesson learned article was issued, procedures revised, and staff briefed on hazards. The Laboratory Director communicated to senior management his 
expectations of their accountability for safety at the Laboratory, that managers need to be in the laboratories on a regular basis to help instill the importance of safety, and to see that work is being done safely.

\section{Analysis of Laboratory Events}

In addition to the review for the electrical shock event, PNNL IO was commissioned to review several other events over the last 12 months to determine if any systemic issues were developing (Report \#IO-2003-15). Laboratory managers want greater clarity about safety performance trends so they can improve and sustain performance. IO concluded that the most common failure mode in the safety program is that Laboratory staff do not recognize hazards or risks due to experiential bias. That is, staff are not able to recognize a hazard as such, due to the apparently unchanging presence of the hazard. The report also noted that PNNL management is responsive to correcting hazards and unsafe conditions in a timely manner when issues are brought to their attention.

PNNL senior management has reviewed the IO conclusions and has responded to the PNNL Laboratory Director via formal correspondence including planned corrective measures. Actions are being tracked in the Assessment Tracking System and will be evaluated as to their effectiveness.

\section{Continual Improvement}

A corporate review of PNNL's Integrated Safety Management System (ISMS) was conducted in August to determine implementation effectiveness. The results identified that PNNL has strong systems and processes in place, and the effective implementation of these systems is attributed to strong line management commitment to safety combined with highly knowledgeable and motivated staff. There were no significant findings that would have the potential to impact the DOE mission, worker safety, health, environmental protection or national security.

PNNL's Independent Oversight group conducted an audit of our environmental management system (EMS). The results were positive, indicating the
EMS is maturing and improving (see report \#IO-200317), and affirmed our ability to retain ISO14001 registration. A surveillance audit by the ISO registrar (NSF-ISR) is scheduled for early October to review our EMS to ISO14001 protocols.

We continued to make improvements to the management systems, processes and tools under the ESH\&Q Directorate purview. Details about these enhancements can be found in module 2.3.1a.

Table 2.1.1. ESH\&Q performance continues to meet target goals, demonstrating the Laboratory's commitment to staff Safety, Health and the protection of the Environment.

\begin{tabular}{|c|c|c|}
\hline Performance Measure & Target Goals & $\begin{array}{l}\text { FY2003 } \\
\text { Actuals }\end{array}$ \\
\hline $\begin{array}{l}\text { Total Recordable Case Rate } \\
\text { w/Subcontractors [36 Month } \\
\text { Rolling Cumulative] }\end{array}$ & $\begin{array}{c}<2.5 \text { cases/ } \\
200 k \text { work hrs }\end{array}$ & 2.0 \\
\hline $\begin{array}{l}\text { Days Away, Restricted or } \\
\text { Transferred w/Subcontractors } \\
\text { [36 Month Rolling } \\
\text { Cumulative] }\end{array}$ & $\begin{array}{c}<1.1 \text { cases/ } \\
200 \mathrm{k} \text { work hrs }\end{array}$ & 1.0 \\
\hline $\begin{array}{l}\text { Voluntary Protection Program } \\
\text { (VPP) Annual Self-Evaluation } \\
\text { Rating }\end{array}$ & Rating of $9-12$ & 9.5 \\
\hline $\begin{array}{l}\text { Conformance of } \\
\text { Environmental Management } \\
\text { System to ISO14001 } \\
\text { Standard }\end{array}$ & $\begin{array}{l}\text { Retain ISO } 14001 \\
\text { registration }\end{array}$ & Met target \\
\hline $\begin{array}{l}\text { Reportable Occurrences of } \\
\text { Release to the Environment }\end{array}$ & $<2$ events & 0 \\
\hline $\begin{array}{l}\text { Pollution Prevention (Low } \\
\text { Level Radioactive Waste } \\
\text { Gen) }\end{array}$ & $\begin{array}{l}\text { Less than } \\
224 \text { Cubic } \\
\text { meters/yr }\end{array}$ & $\begin{array}{c}93.6 \text { cubic } \\
\text { meters } \\
\text { YTD }\end{array}$ \\
\hline $\begin{array}{l}\text { Pollution Prevention } \\
\text { (Haz Waste Gen) }\end{array}$ & $\begin{array}{l}\text { Less than } \\
11.9 \mathrm{MT} / \mathrm{yr}\end{array}$ & 8.5 MT YTD \\
\hline $\begin{array}{l}\text { Occurrences of Spread of } \\
\text { Contamination }\end{array}$ & $<3$ events & 0 \\
\hline
\end{tabular}

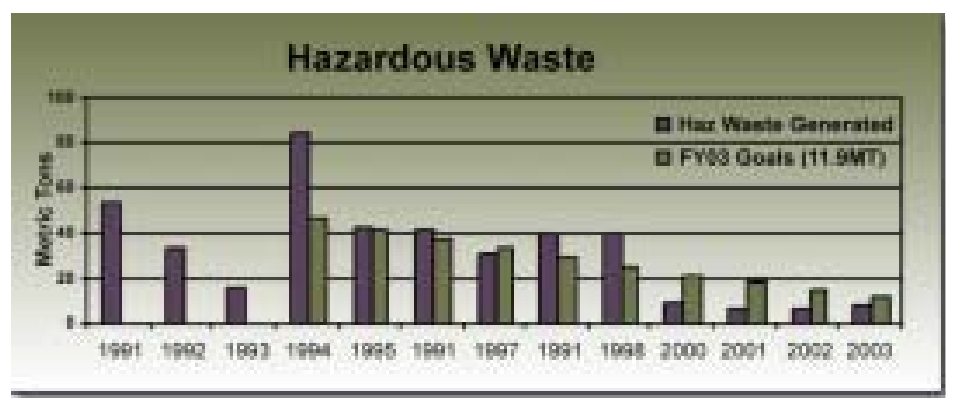

Figure 2.1.1. Reducing our Hazardous Waste output supports the Laboratory's environmental stewardship goal by decreasing our environmental footprint. 


\subsubsection{Performance Against Financial Management Metrics \\ PNNL's FY2003 successful business performance reflects continual cost improvement and investment for the future in spite of delayed programmatic funding.}

Strong financial management performance in spite of a five-month continuing resolution (CR) confirms PNNL's strong internal controls. Since the bar was set high in this area by CR, without the delay we would have achieved outstanding performance. Overhead cost was effectively managed, as indicated by the outstanding rating on overhead as a percent of the charge out rate; but uncertainty in federal budgets during the first quarter of the fiscal year resulted in a slow start for direct FTEs and consequently less improvement on the labor cost multiplier than otherwise expected.

\section{Overhead Cost as a Percentage of Charge-out Rate}

Overheads as a percentage of labor charge out rates measures the relative efficiency of PNNL's overhead structure and approach. We continue to make progress toward reducing the overhead component of our labor rates to below 50\%, with an outstanding result in FY2003 of $50.4 \%$. This means that roughly half of each dollar paid for a staff member's time goes directly to salary and benefits. PNNL is committed to pricing and rate decisions that will put more than half of each dollar into direct costs.

\section{Total Overhead Cost Expressed} as a Multiplier

Overhead cost applied to labor expressed as a multiplier of labor cost, a business indicator used by other DOE Laboratories, allows PNNL to benchmark and continue to press for improvement. For every dollar of directly charged cost to a client, an overhead amount is applied. PNNL reduced this multiplier by nearly $1 \%$, in FY2003, resulting in only a good rating for this measure. The overall composite multiplier for the Laboratory, however, was reduced by over 2\%, reflecting PNNL management's commitment to overall cost productivity improvement.

\section{Direct FTEs as a Percent of Laboratory Total FTEs}

Direct FTEs as a percent of total Laboratory FTEs is a further indicator of our efficiency in deploying Laboratory resources. To the extent that our activities are increasingly funded by our clients, we have a viable and relevant business base. In FY2003, 50.4\% of all FTEs at PNNL were funded by direct client programs. The aforementioned funding delay caused this metric to come in at excellent rather than outstanding; even so, we did make progress in increasing the percentage of PNNL staff funded by programs over FY2002 performance.

\section{Other Improvements Made}

In FY2003, we made significant improvement in work authorization and funds control. For more details, please see module 2.3.1b. As a result of the funds control issues identified in FY2002, BSS completed an in-depth review of business and operational risks and developed action plans to address risk areas. We developed an action tracking system to ensure appropriate level of attention and resolution to all risk-related matters requiring significant management involvement. A key objective for FY2004 is to continue to institutionalize the risk assessment and mitigation process.

\section{Institutionalizing a Culture Focused on Improvement}

The above business indicators focus PNNL management on improving cost performance as they make decisions on pricing rates and investment levels. By keeping these metrics in front of Laboratory management, we have helped make every year better than the previous one. While the improvement is sometimes less than hoped, the metrics keep us moving in the right direction.

To support continued progress in these metrics, we have formed a Cost Productivity Review (CPR) Team to find, over the next three years, more resources that can be 
redirected to accomplish the Laboratory's strategic objectives or reduce indirect rates. Examples of significant cost items currently being evaluated for reduction include lease costs, site services, and acquisitions. The CPR strategy will sensitize the Laboratory to the need for resource allocation decisions based on facts and data consistent with overall Laboratory strategy. Since Laboratory staff's ability to prioritize their efforts is fundamental to efficient and effective operations, a clear articulation of the Lab's vision statement is instrumental in this regard. The strategy map currently under development will be a key input to this activity, clearly communicating Lab strategy and illuminating the context for tradeoffs among alternative activities.

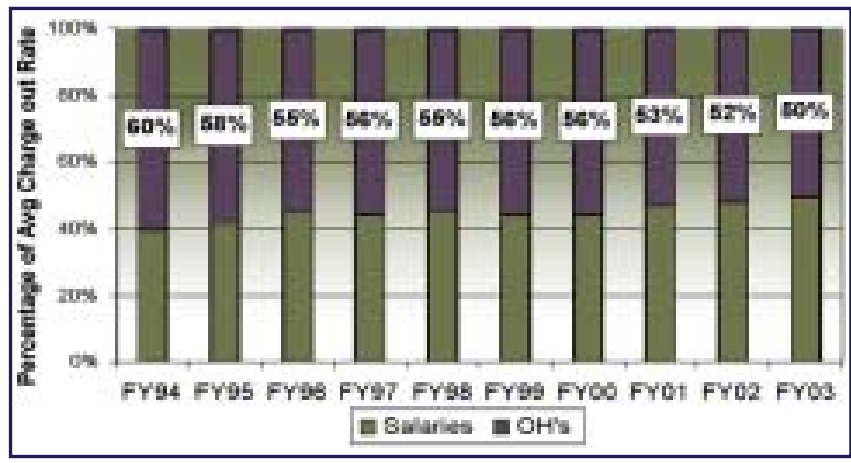

Figure 2.1.2a. PNNL continues to reduce overhead rates, resulting in an outstanding rating relative to overheads as a percent of charge out rates.

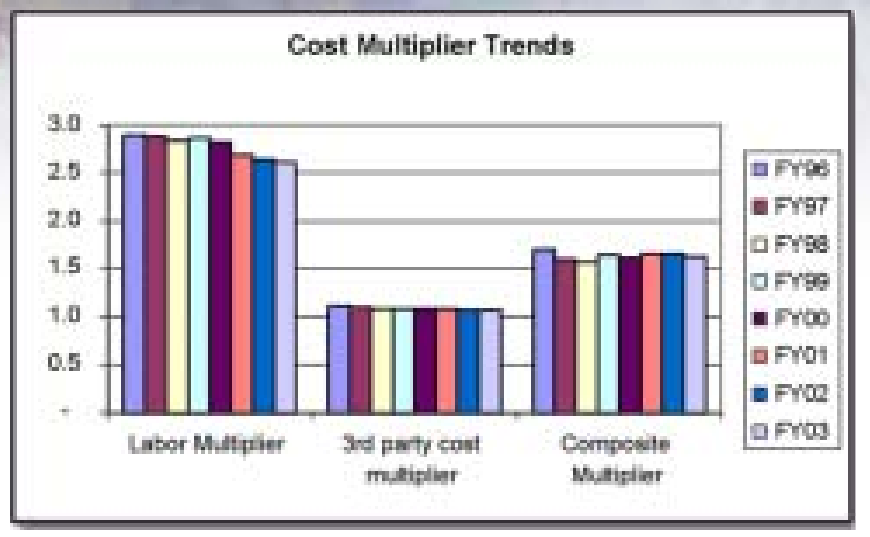

Figure 2.1.2b. Overhead costs expressed as a multiplier on direct costs decrease by nearly $1 \%$, resulting in only a good rating for this measure.

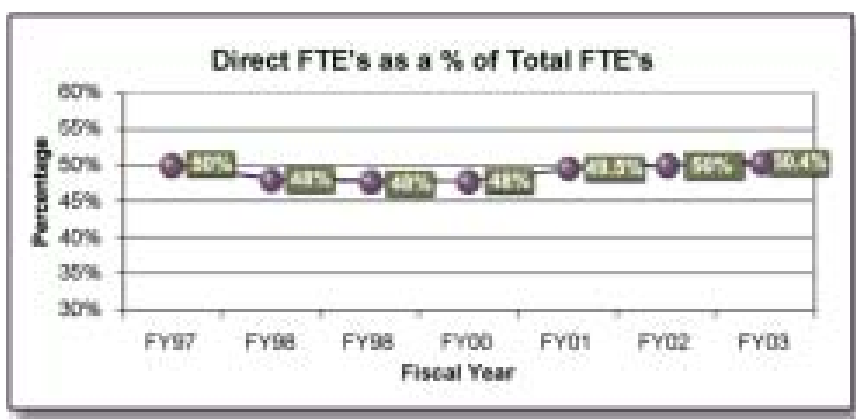

Figure 2.1.2c. The number of staff funded directly by clients continues to increase relative to staff funded by Laboratory overheads, translating to an excellent rating on this measure. 


\title{
2.1.3 Sustaining the Effectiveness of Integrated Safeguards and Security
}

\author{
In FY2003, PNNL achieved a composite average \\ performance for the $11 \mathrm{SAS}$ processes of 3.6, for a \\ rating of outstanding.
}

\section{External Evaluations Highlight Maturity of ISSM Program}

The Laboratory's performance in Safeguards and Security (SAS) programmatic areas at protecting assets and compliance, as measured by external evaluations and performance against agreed upon corrective action plans, met expectations. All external evaluations of SAS, with one exception, resulted in satisfactory (the highest possible rating) or greater composite ratings.

The DOE Office of Independent Oversight and Performance Assurance (OA) inspected the SAS programs (at both Hanford and PNNL) during the third quarter. The SAS and Classified Cyber Security areas received "effective performance" ratings. OA inspectors called out our ISSM program as a highlight, confirming the success of our efforts to integrate SAS into the PNNL culture:

"The PNNL implementation of ISSM is one of the leaders in DOE at this point."

"The Laboratory exhibits a significant culture shift from a view that security is an impediment to research to the recognition that it is a necessary component of a quality research and development program in the national security area. PNNL represents an ISSM success story for the Department and an example of the benefits of ISSM within a national laboratory environment."

The Unclassified Cyber Security program area, however, did receive a "significant weakness" rating, and we established a task team to develop and implement corrective actions. Positive aspects of this rating have led to the validation that PNNL's long-term plans are on track and the right way to proceed. Immediate actions primarily address short-term risks and will result in a stronger PNNL Unclassified Cyber Security program.

DOE-RL conducted the Periodic Security Survey of Battelle in November 2002, focusing on the extent to which PNNL complied with applicable DOE directives to ensure protection of government property and information. PNNL SAS received a satisfactory rating.
DOE-HQ (Office of CIO for Cyber Security) conducted inspections of the COMSEC, TEMPEST, and Protected Transmission Systems in May. There were no findings in any of these areas.

The Information Classification and Control Policy (ICCP) office conducted an appraisal of the Classification program. The overall rating received was "meets expectations."

\section{SAS Performance Matches Reputation}

The Laboratory continues to demonstrate its ability to identify, report, and, as necessary, mitigate emerging threats. Line organizations ensured the number of significant incidents of a security concern within the control of Battelle with impacts upon the national security, defense, or foreign relations of the United States were minimized and mitigated. Corrective actions for identified threats or issues were rapidly developed and implemented. SAS "internal" incidents remain below average (14 for FY2003 vs. 25 in FY2002). There was one externally reportable incident.

\section{Management is Committed to SAS}

Line organization staff and management's currency with SAS training requirements met or exceeded expectations. The average completion rate of SAS courses has risen from last year $(96.5 \%$ for FY2003 vs. $95.4 \%$ for FY2002), reflecting the Laboratory's commitment to SAS and ensuring that training and knowledge are commensurate with assigned responsibilities.

\section{A Focus on SAS Process Efficiencies}

Safeguards and Security Services (SASS) continues to streamline and automate processes that help Laboratory management and staff with their daily SAS responsibilities. The Entrust digital signature was implemented in all SAS request and processing applications. Progress with the Role Based Access Control (RBAC) process is ongoing. RBAC will streamline internal processes for Part I: Performance Against Critical Outcomes2.0 Management and Opeations 
SASS activities and other Laboratory systems by assigning user privileges based on a centrally managed role (vs. name). The SASS workflow component involving our electronic routing and approval engine (a common workflow component for web-based SASS request/approval systems) was submitted and selected as one of the finalists for the 2003 Government Technology Lead- ership Awards (GTLA): The Gracies. The GTLAs recognize projects making exceptional contributions to mission accomplishment, cost effectiveness, and public service. It highlights technology's growing potential for improving government operations. Award status should be announced the end of September 2003.

Table 2.1.3. A composite score of 3.6 confirms PNNL's rating of outstanding.

\begin{tabular}{|l|c|c|c|}
\hline SAS Performance Indicators & Outstanding Goal & FY2003 Performance & Points \\
\hline $\begin{array}{l}\text { Monthly Security Events } \\
\text { (monthly average \# of events) }\end{array}$ & $<3$ & 1.17 & 4 \\
$\begin{array}{l}\text { Self-Reported Events } \\
\text { (monthly \% of incidents reported) } \\
\text { Publications Reviewed for }\end{array}$ & $>80 \%$ & $100 \%$ & 4 \\
$\begin{array}{l}\text { Classification/UCNI } \\
\text { (\% publications reviewed) }\end{array}$ & $>4 \%$ & $10.9 \%$ & 4 \\
$\begin{array}{l}\text { Unclassified Cyber Security } \\
\text { (\% reported on time) }\end{array}$ & $100 \%$ & $100 \%$ & 4 \\
$\begin{array}{l}\text { Employee and Management } \\
\text { Awareness(\% training complete) } \\
\text { Security Clearances - }\end{array}$ & $>95 \%$ & $96.5 \%$ & 4 \\
$\begin{array}{l}\text { Selective Representative } \\
\text { (\% submitted monthly) }\end{array}$ & $100 \%$ & $95.3 \%$ & 3 \\
$\begin{array}{l}\text { FNVA Processing Time } \\
\text { (\% submitted within 10 days) }\end{array}$ & $>80 \%$ & $75.2 \%$ & 3 \\
$\begin{array}{l}\text { Foreign Travel Trip Reports } \\
\text { (\% past due) } \\
\text { MC\&A Performance Tests } \\
\text { (Performance tests performed) }\end{array}$ & $100 \%$ & $9.7 \%$ & 4 \\
$\begin{array}{l}\text { Self Assessments } \\
\text { (annual assessments/ } \\
\text { corrective actions completed) } \\
\text { External Evaluations } \\
\text { (Satisfactory rating/corrective } \\
\text { actions completed) }\end{array}$ & $20 \%$ & Average Annual Score & \\
\hline
\end{tabular}




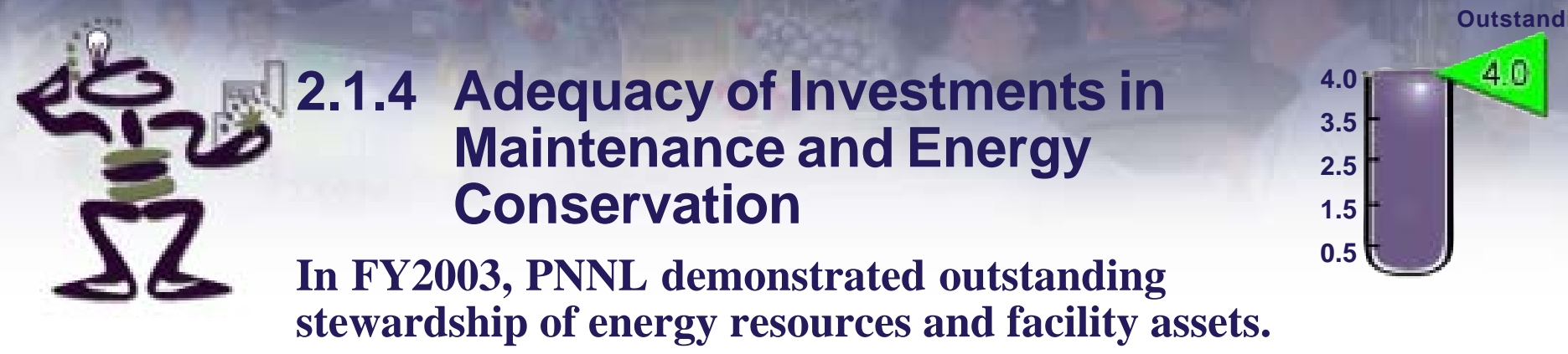

FY2003 was a celebratory year, accented by awards and public recognition for achievements in energy and water conservation. Our customer service commitment has been palpable and relentless, and the numbers show it. Customer survey results across service lines were consistently strong all year: User Housing Facility (93\%), Convenience Services (92\%), Core Teams (96\%), and Moves (91\%). We continued a track record of outstanding stewardship of the physical plant.

Despite economic challenges, we met target levels of maintenance investment while sustaining a high level of facility availability/reliability.

\section{Stewardship Index}

Customer satisfaction-highest ever. Over the last three years we have placed great emphasis on customer service and our ability to be more productive with technology to achieve improved and more dependable performance. Customer survey results across service lines were consistently strong all year. Facilities \& Operations received the highest ever annual survey customer satisfaction rating-3.8.

Facility reliability/availability-365/24/7. In FY2003, we continued our track record of outstanding stewardship of the physical plant. Despite economic challenges, we met target levels of maintenance and energy investment while sustaining a high level of facility reliability and availability.

Maintenance investment - strategic maintenance investments achieved. PNNL's efforts to reduce energy use, implement risk management decisionsupport tools, and improve productivity through technology allowed us to identify $\$ 900,000$ in cost savings, most of which was used to make investments for the future of the Laboratory. The move of 3720 laboratory operations to 325 , engineering support for the design and construction of a future bio-processing laboratory in partnership with Washington State University, a study of utility alternatives to support development of the modern research campus, and the conversion of the Sequim facility warehouse to offices.
As displayed in Figure 2.1.4, continued strong performance in three key process measures (dispatch cycle time, preventive maintenance completion percentage, and need by date percentage) validates that our approach to work planning and control is effective and efficient: we do the right things, and we do them well.

\section{Outstanding Energy Conservation}

PNNL met all of the 7 milestones under this sub-indicator. FY2003 was another outstanding year for energy conservation. PNNL has achieved a dramatic reduction in energy use for office buildings. We exceeded our target of 2\% reduction from the previous year; achieving $2.7 \%$. These cost-saving improvements allow the laboratory to increase support for key initiatives. In addition, we use these savings as a source of funds to diversify our energy portfolio with a greater share of renewable power. In FY2003, we successfully acquired more than $13 \%$ renewable power, exceeding DOE's goal for 2010 by $83 \%$.

FY2003 saw a spate of awards and public recognition. PNNL became the first Office of Science Laboratory to earn two Energy Star ${ }^{\circledR}$ building labels from the Environmental Protection Agency (EPA). We were also recognized by the Association of Washington Businesses for our success in environmental resource recovery. Additionally, PNNL received two Department of Energy Management Awards honoring the Laboratory's outstanding achievements in energy and water management. PNNL's Facility Energy Manager was also designated an Energy Champion through the Federal Energy Management Program's You have the Power campaign.

Finally in this area, during FY2003, Facilities \& Operations implemented a sustainable Facility Management policy and plan to promote energy conservation, acquire technology improvements to increase energy efficiency and conserve water, and transition to dependable, affordable, and environmentally sound sources of energy. PNNL's demonstrated excellence in this area earned us the International Facility Management Association (IFMA) Facility Management Achievement Award (to be awarded in October 2003). 


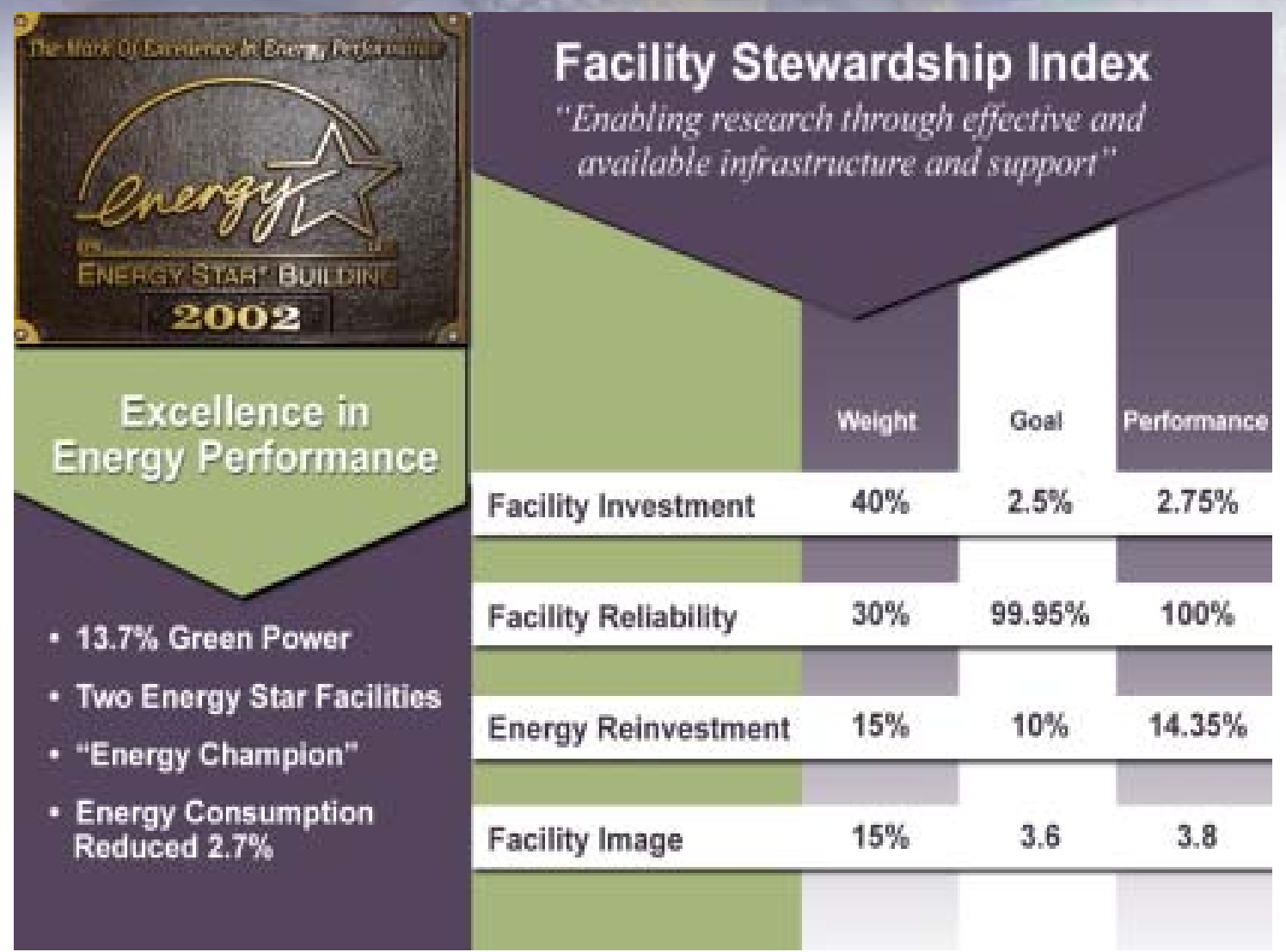

Figure 2.1.4. PNNL exceeded all maintenance and energy conservation expectations in FY2003; achieving a Stewardship Index of 1.0. 


\subsubsection{Enhancing EMSL to Support the User Community}

PNNL has met all $900 \mathrm{MHz}$ milestones under its
control and has brought to full operational status
one of the most powerful supercomputers in the world.

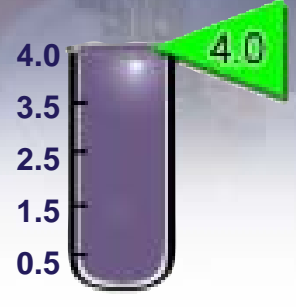

The Oxford $900 \mathrm{MHz}$ NMR magnet is at full field. Repairs and resultant improvements made to the magnet were successful at dramatically reducing its helium (He) consumption. A special-purpose $900 \mathrm{MHz}$ triple resonance probe has been designed, built, and tested. The probe will be employed by EMSL users and grand-challenge scientific research. The Hewlett-Packard Supercomputer, one of the most powerful computers on the planet (11+ teraflop), has been successfully installed, brought to full operational status, and is available to the user community.

\section{Mhz Magnet/Helium Recovery System}

The $900 \mathrm{MHz}$ magnet, delivered in March 2002, brought to field in June 2002 but de-energized in December 2002, underwent significant high-risk repair and refurbishment between January and September of FY2003. These repairs, necessitated by suspected contamination in the magnet ultra-cold cooling system and following contentious negotiations with Oxford Instruments, were completed in early September. At year end, the magnet is performing better than ever experienced, and is about to enter formal and final acceptance testing. The associated EMSL facility liquid He recovery system was also commissioned and upgraded. Continuous recovery was first demonstrated this year, with a capacity increase of almost $400 \%$ due to the installation of new equipment. Concomitantly, the magnet repair effort resulted in a $30 \%$ He consumption reduction. Together, these developments provide for adequate backup capability as well as $\mathrm{He}$ liquefaction surge capacity for the future. This NMR system is one of the largest of its kind, and will draw scientific users from around the world.

\section{Special-Purpose Probes}

A custom solids NMR probe was designed and built at EMSL for $900 \mathrm{MHz}$ NMR use. It enabled the first demonstration of solids NMR at this field. The probe, a double resonance static model, showed outstanding performance in use with a narrow-bore $900 \mathrm{MHz}$ magnet that was made available to us by Oxford Instruments while the EMSL magnet undergoes installation \& repair. The probe will become part of the EMSL user facility probe pool, allowing external users access to high-field solids NMR capability. A user publication (“A 4-mm Probe for 13 C CP/MAS NMR of Solids at 21.15 T”, H. J. Jakobsen, P. Daugaard, E. Hald, D. Rice, E. Kupce, and P. D. Ellis, J. Magnetic Resonance) was produced with the seminal results obtained.

\section{HP Supercomputer}

The IBM Supercomputer was decommissioned, and full operations - Phase1 of the HP supercomputer began before the end of 2002. The HP brings a four-fold increase in computational power for the scientists of the EMSL's Molecular Science Computing Facility. Phase 1 of the HP supercomputer served as the production system during the first half of 2003; by June, the interim Phase 2 system was fully operational and the workload transitioned over to that system. The interim Phase 2 system and the Phase 1 system were combined in late July to result in an 11.8TeraFLOP $(11,800,000,000$ Floating Point Operations Per Second) system. After passing all operational milestones, the supercomputer was fully accepted during FY2003. At the time of deployment the system was the fastest unclassified computer in the United States, representing a major success story for the DOE Office of Science and Pacific Northwest National Laboratory. 


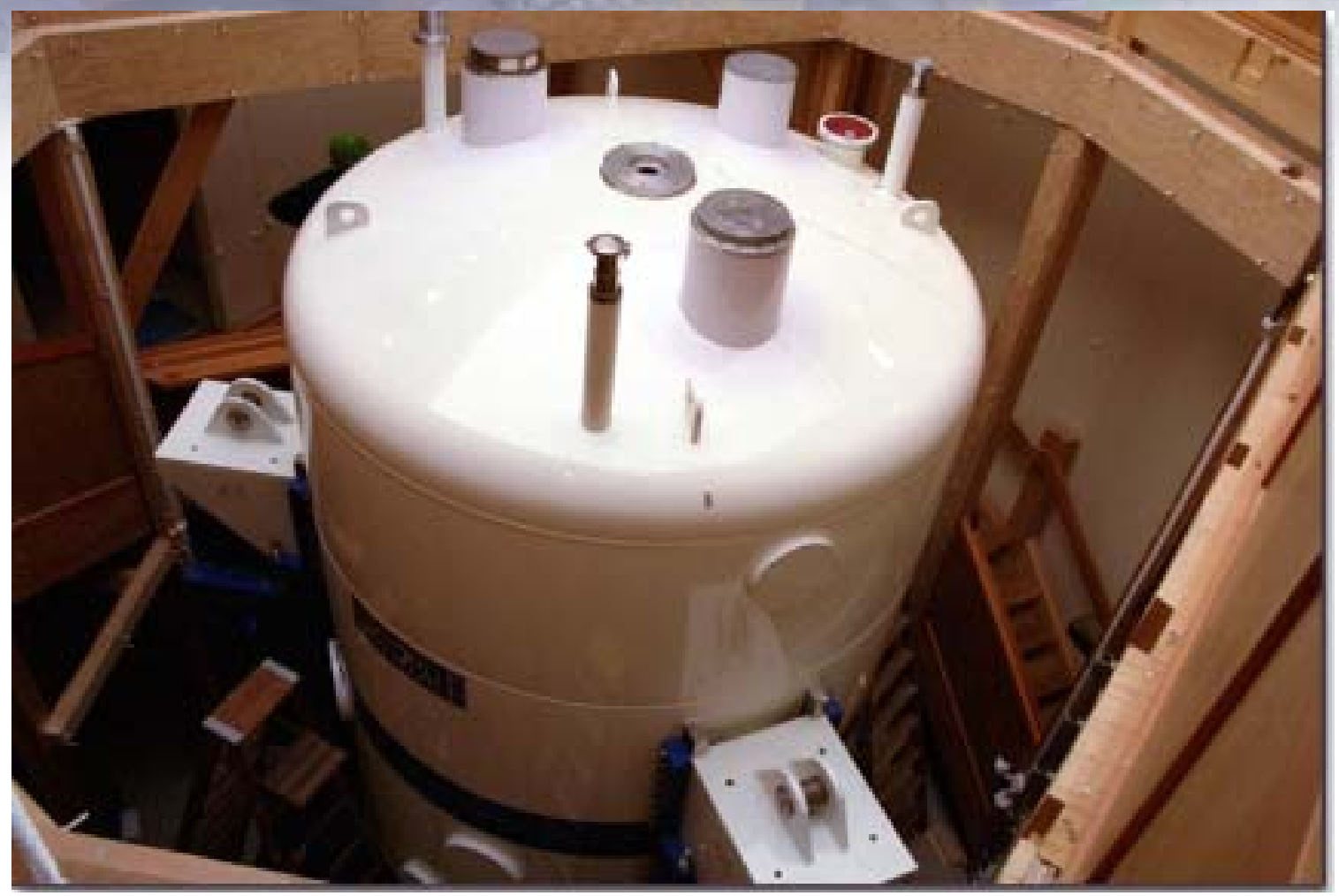

Figure 2.2.1a. $900 \mathrm{MHz}$ NMR, world's largest NMR

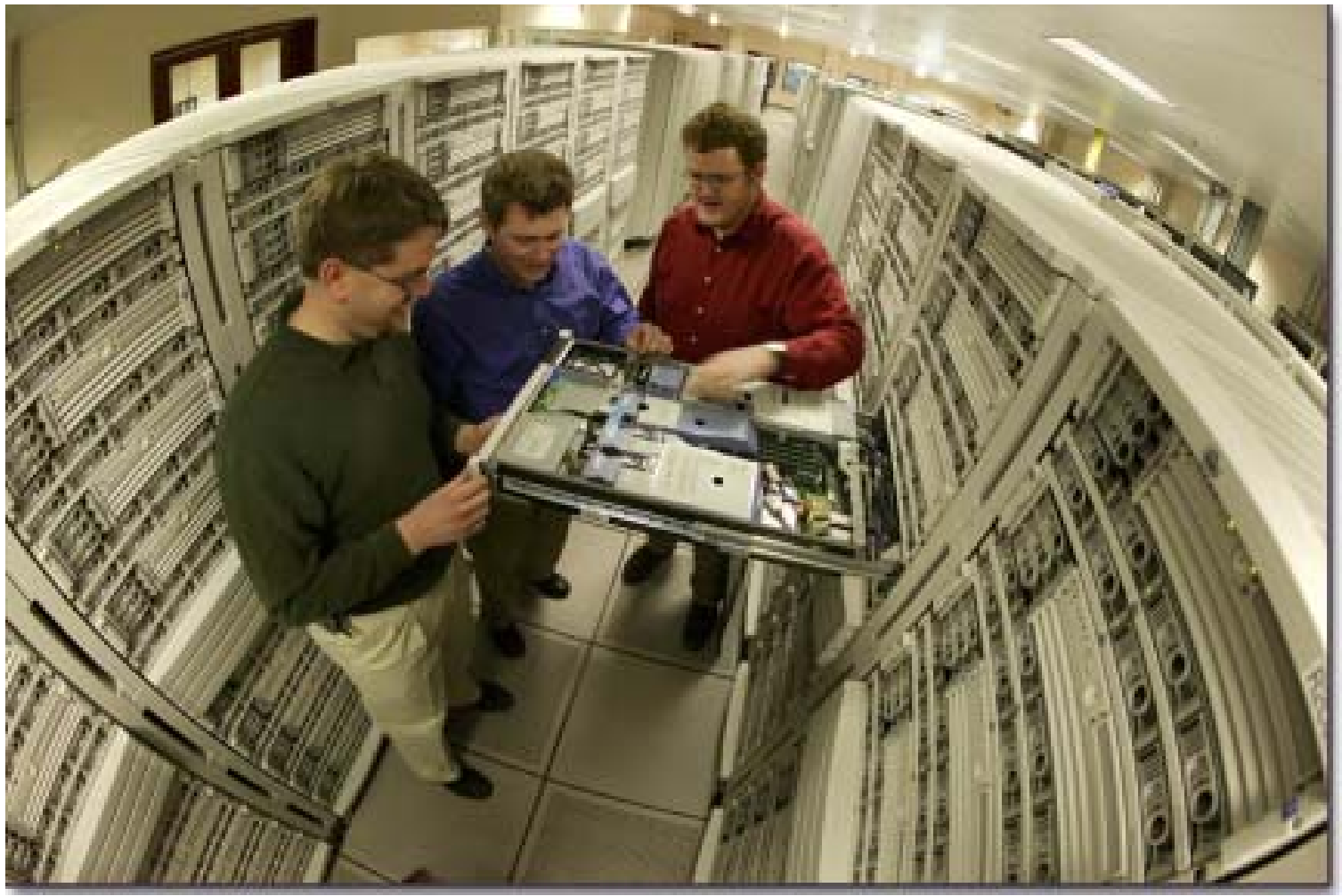

Figure 2.2.1b. $11+$ Teraflops - One of the fastest on the planet 


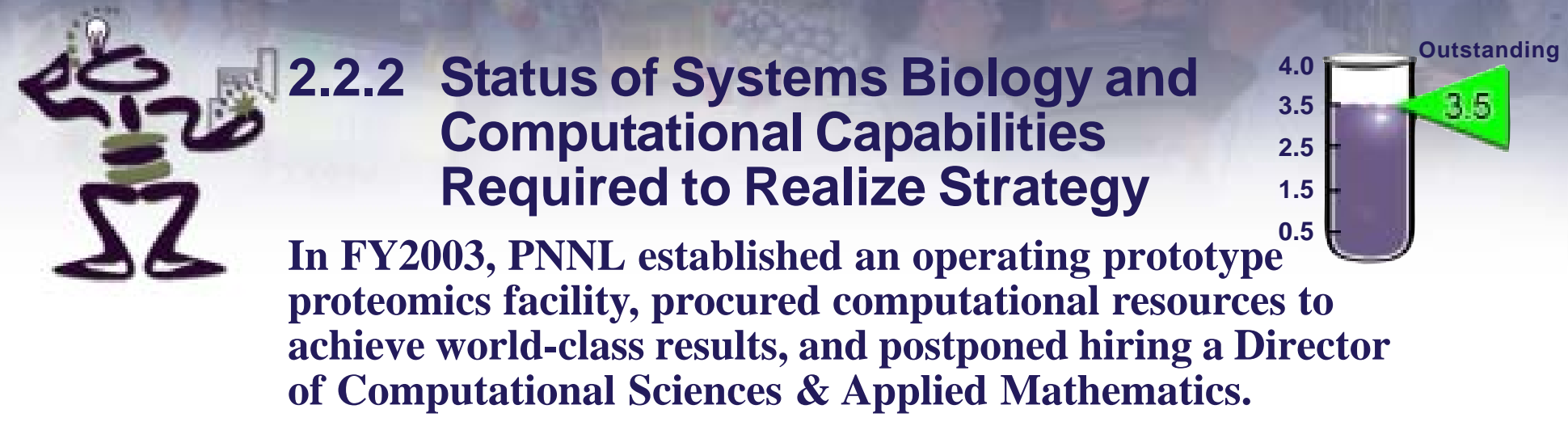

PNNL will play a significant role in unlocking the mysteries of proteins. The establishment of a prototype proteomics laboratory (LSL-II \& EMSL) represents a significant achievement in support of these efforts and the Biomolecular Systems Initiative (BSI). The PNNL Computational Requirements Task Force has evaluated and captured the high-end computational needs for PNNL $\mathrm{R} \& \mathrm{D}$ in our major mission areas. A general-purpose high-performance computer has been procured and installed. Hiring a Division Director of Computational Sciences was postponed.

\section{Proteomics}

In support of the Department of Energy's Genomes to Life research and PNNL's systems biology program, the Lab established a Prototype Sample Processing and Proteomics Facility. Key deliverables included 1), research equipment obtained, installed, and made operational in the LSL-II space; 2), staff relocated and operational in the new facility; and 3), facility became an operating and productive. By the end of the fiscal year, we successfully characterized five protein complexes, exceeding the outstanding performance rating by $20 \%$.

\section{Requirements Assessment/ Strategic Procurement}

PNNL established the Computational Requirements Task Force to capture and summarize the high-end computational needs for R\&D in our major mission areas (assessment). We focused specifically on the needs of our computationally intensive science and engineering programs, including modeling, simulation, and data analysis, in both unclassified and classified domains. Key findings include the following needs:

- High-end computers including large-scale storage, i.e., systems located in dedicated computer rooms, rather than an office or lab, and the local data storage associated with them;
- High-speed data network connections from PNNL to major clients, and other computational resources including large data storage facilities; and

- Facilities to house these high-end computers, i.e., computer rooms, and personnel to manage and operate them.

A significant outcome of the requirements activities is the establishment of a PNNL Advanced Cluster Computing strategy that will be deployed in FY2004. This strategy will allow high-performance computing to be made available to a greater number of scientific domains across PNNL.

PNNL has purchased an SGI Altix for general purpose high performance computing. The system, open to all staff doing 1830 work, runs a single copy of Linux over 128 Intel Itanium 2 processors running at $1.5 \mathrm{GHz}$. The system boasts $256 \mathrm{~GB}$ of RAM and 1/2TB of disk space .

\section{Director Hire - Computational Sciences \& Applied Mathematics}

PNNL's senior management chartered a staff team to identify potential candidates for the Division Director for Computational Sciences \& Applied Mathematics position. Of several candidates, the best was selected, and in May 2003 PNNL extended an offer, which unfortunately was declined. The team began another extensive search, and was ready to extend an offer, when senior leadership changes were announced at the Lab and it was decided not to fill the position until selection of a new ALD for FSD. 


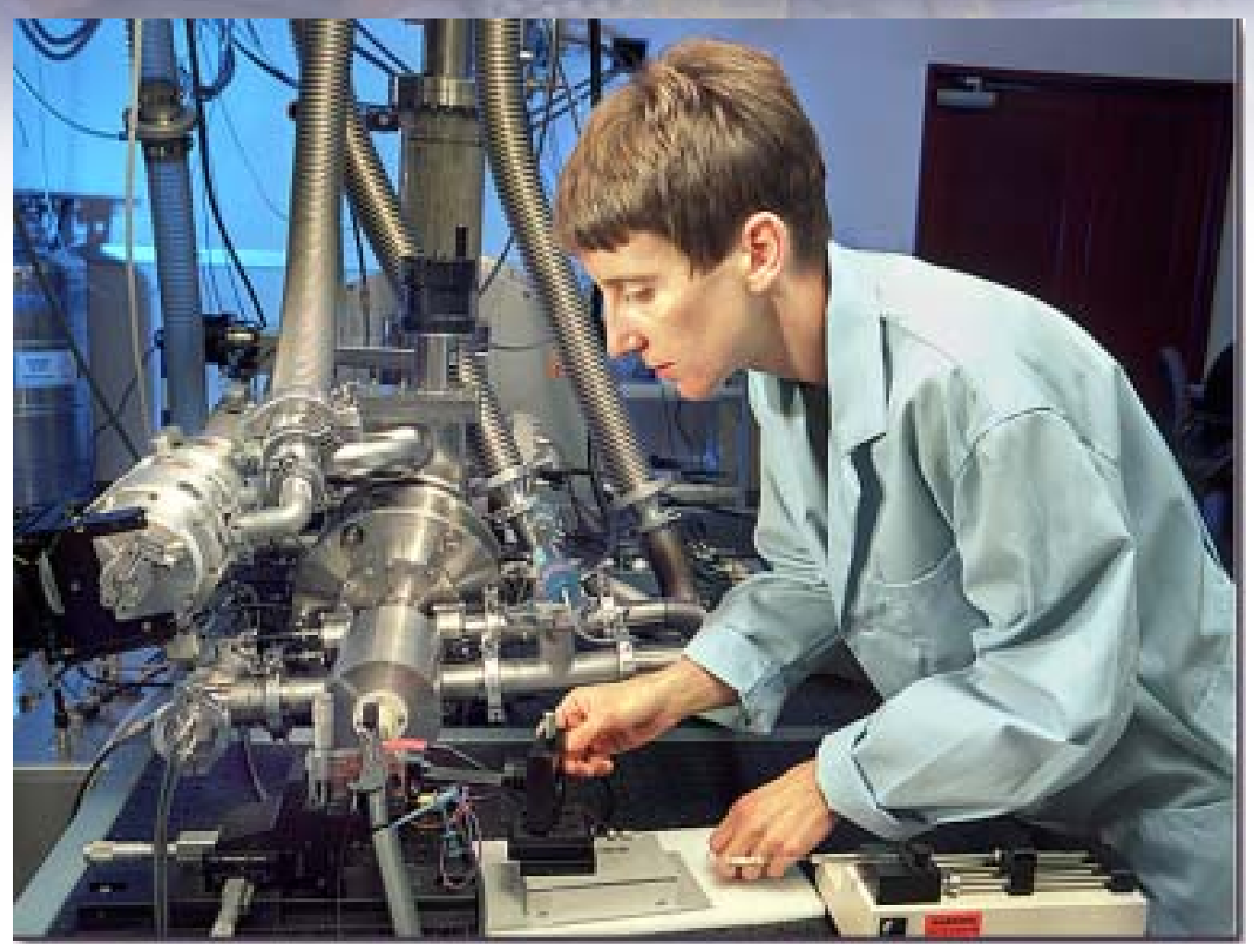

Figure 2.2.2a. Understanding proteins and how they work will greatly improve medicine, health care, and environmental cleanup.

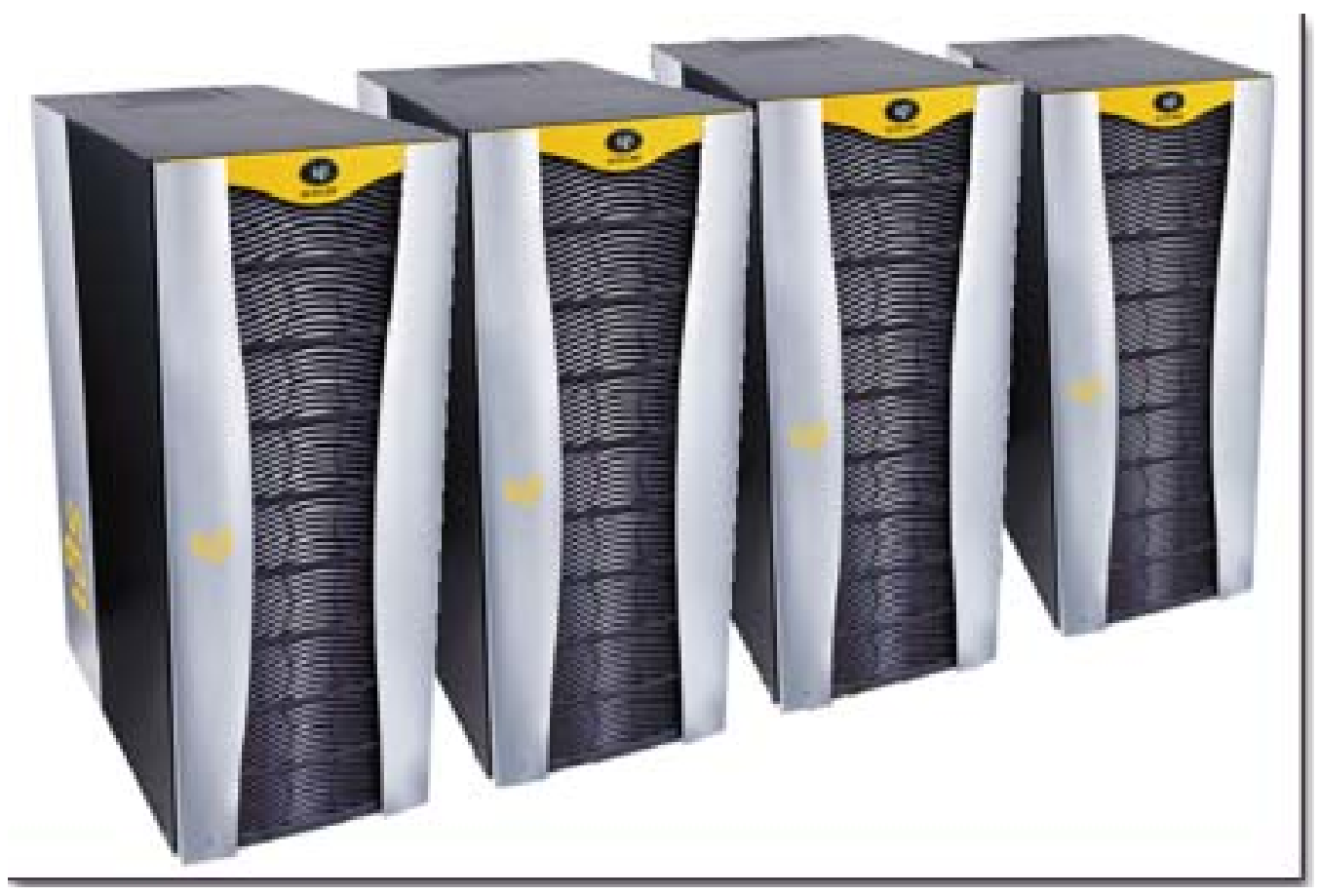

Figure 2.2.2b. General Purpose High Performance Computer for PNNL. Colony 2: SGI Altix; the world's first 128-way Linux SMP. 

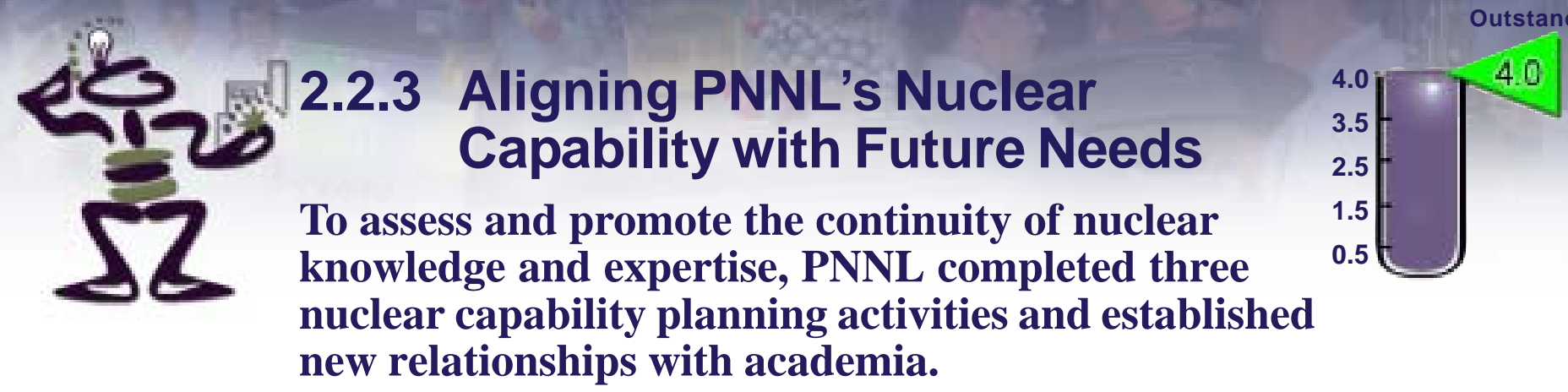

PNNL has a rich history of significant contributions to national and international advances in nuclear science and technology. Its nuclear capability is deployed in the three critical areas of nuclear legacy; the threat of nuclear, biological, and chemical (NBC) weapons proliferation; and energy generation. To retain nuclear science and technology as part of PNNL's multi-program signature, facility and staff capabilities must be sustained and new talent recruited and developed. PNNL nuclear-related capabilities were assessed with a look to the future. We analyzed staff, equipment, and facility needs against potential future mission requirements. We also enhanced university ties on behalf of developing the next generation of nuclear scientists and engineers.

\section{Integrated Nuclear Strategy}

In January 2003, a draft integrated nuclear capability assessment was discussed with PNNL leadership. It found PNNL's nuclear capability contributed significantly to the development of new knowledge and solutions and had significant growth potential. Key strategy recommendations include:

- Implement a new integrated view of PNNL's nuclear business, based on three theme areas: 1) Legacy, 2) NBC threats, and 3) Energy.

- Refocus the Advanced Nuclear Science and Technology Initiative and associated capabilities to these strategic areas.

- Establish a Radiological Detection and Analysis Center to provide direction for new and sustainable radiological capabilities and programs in support of national security and homeland defense.

- Articulate the relative importance (via Laboratory leadership) of the Lab's radiological/nuclear capabilities for the Lab's future.

- Define, maintain, and acquire required facility and staff capabilities.

The Integrated Nuclear Strategy was submitted to the PNNL Management Council March 28, 2003. Mike
Lawrence, Associate Laboratory Director for Energy Science and Technology, forwarded the Strategy to Paul Kruger, Associate Manager, Pacific Northwest Site Office, by letter dated April 9, 2003, for information and for follow-up PNNL/PNSO discussions.

\section{Critical Decision 0, Mission Justification and Facility Requirements}

A June 12, 2003 meeting of the Department of Energy's Office of Science and Office of Environmental Management reinforced the importance of identifying facility capabilities required to implement PNNL's Integrated Nuclear Strategy (and other facility needs). Uncertainties associated with the River Corridor clean-up schedule mean that PNNL needs to plan to transition from current 300 Area facilities into replacement facilities as soon as possible. Mission-driven needs determine the trade-offs in planning the transition of 300 Area capabilities, many of which contribute to PNNL's Integrated Nuclear Strategy. The draft Mission Need for PNNL 300 Area Transition Facilities: Retaining Core Science Research Capability While Enabling Accelerated Clean-up (CD-0) summarizes the results of discussions to date on future missions and capability tradeoffs, and sets the stage for defining Integrated Nuclear Strategy facility needs during critical decision one (CD-1). This latter activity resulted in the divesture of some capabilities recommended for retention in the Integrated Nuclear Strategy document, mostly in the area of fuels handling.

The draft CD-0, Mission Need document cited above was submitted to DOE PNSO in September 2003 for review and comment. It was also submitted for external validation by peers (the Red Team) from other national laboratories and advisory representatives to PNNL. The Red Team's extensive September 24, 2003 comments along with DOE comments, will be resolved during FY2004. 


\section{Work-Force Analysis Addressing Key Staff Disciplines}

The Integrated Nuclear Strategy assumes overall staff growth in support of on-going and future integrated nuclear capabilities. Our assessment focused on specialty disciplines unique to our nuclear capability needs. The analysis was completed by a set of nuclear-related technical group leaders, augmented by several senior scientists.

PNNL has an impressive cadre of staff across the key disciplines supporting the Integrated Nuclear Strategy, many with national and international reputations. But this workforce is aging; and, while a few disciplines are not in immediate danger of losing critical, irreplaceable staff, others are. Our geographic location, while attractive to many new staff, deters others. Although competition and other external factors will make recruitment difficult, the single largest challenge is the open issue of replacing our aging nuclear facilities. Actions underway in support of staff recruitment include mentoring programs, a hiring campaign to fill a gap of entry-level staff, and networking with industry and expanding relationships with academia.

\section{New Joint Research and Development Projects with Academia}

During FY2003 PNNL continued, expanded, and added several new programs to develop and recruit students in nuclear science and engineering. PNNL created at least one major new internship program, expanded at least four other student programs, and created at least 22 new student positions in nuclear science and engineering projects.

In addition PNNL continued efforts to strengthen ties with the nation's academic community and to foster future collaborations. Alan Waltar chairs the Western Nuclear Sciences Alliance's External Advisory Committee in CY2003. While no specific R\&D collaborations have been established to date with Alliance universities, the stage is being set. In addition, PNNL's Walt Laity serves as Vice President, Engineering Education, for the American Society of Mechanical Engineers (ASME). Chairing the ASME Board on Engineering Education, made up largely of senior faculty members from several of the nation's major universities, he has recently formed a Task Force to examine the future of mechanical engineering education. Relationships formed via these activities can be very useful to PNNL's recruitment efforts.

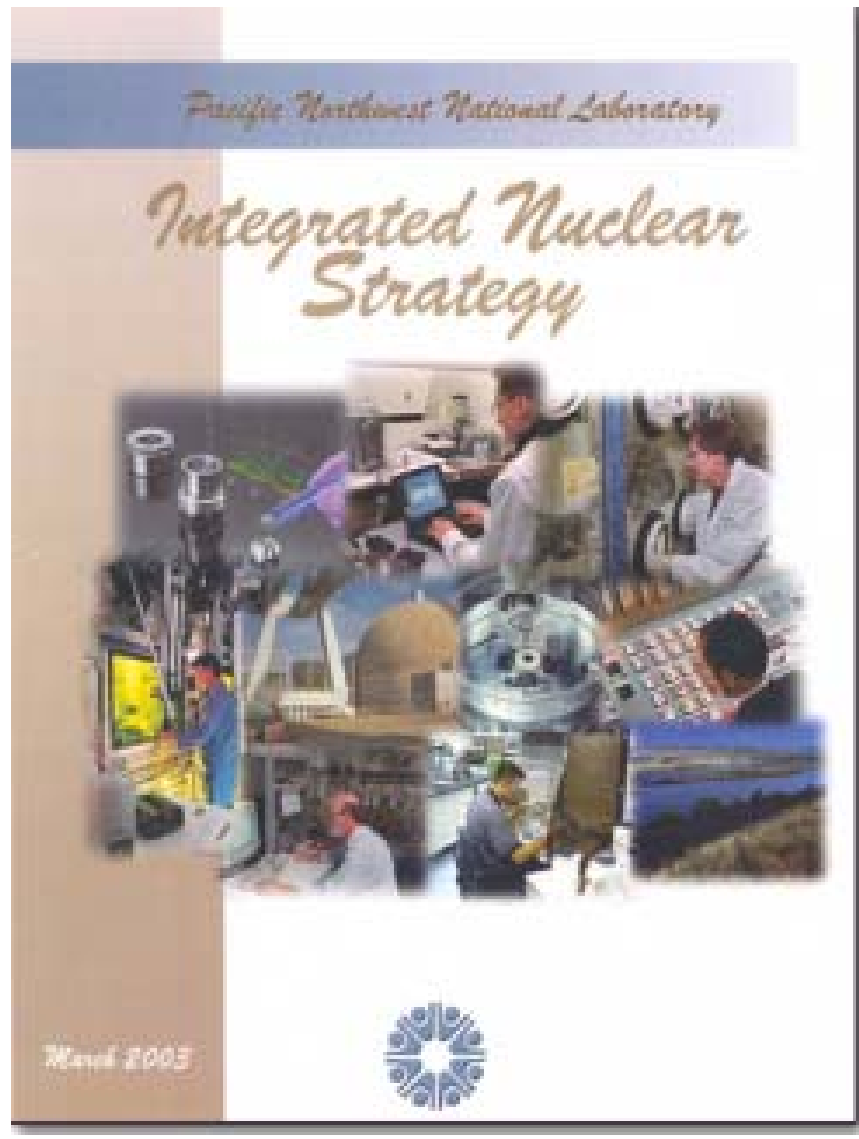

Figure 2.2.3. PNNL's Nuclear Sciences and technology programs and capabilities have been and, we expect, will remain significant elements of our multi-program portfolio. 


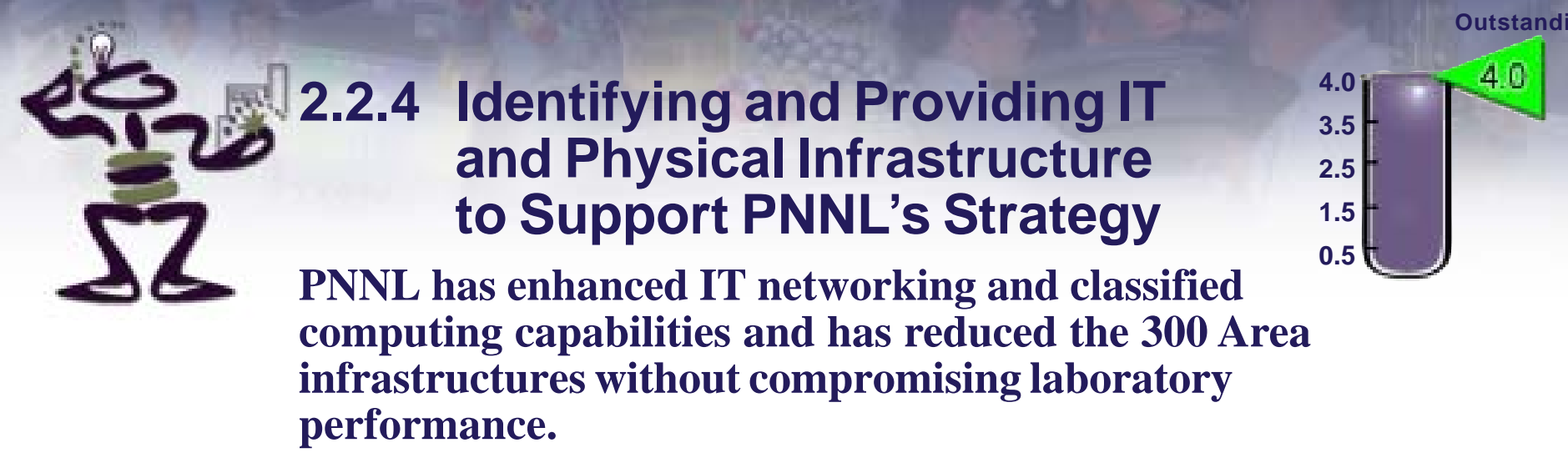

PNNL has installed, tested and implemented OC12, replacing the OC3 connection to the Pacific Northwest Gigapop, to better support regional research collaborations. PNNL has provided local classified computer capabilities, installing a 64-node cluster computer in the 3760 facility. PNNL has given the Department of Energy a white paper articulating the strategy for maintaining continuity of the science mission while supporting the accelerated cleanup.

\section{Enhanced IT Capabilities}

Outstanding: 5 of 6 milestones met. In line with DOE mission needs, PNNL increased capacity and performance of internal and Internet connections to accommodate strategic research collaborations requiring interaction with research data sets and computational requirements of increasing complexity and size with wide geographic dispersion. Internally, the Laboratory deployed 768 additional Gigabit Ethernet ports for connecting highend workstation and servers to the PNNL network (total over 1100). PNNL also upgraded its Internet border routers and implemented a distributed firewall complex to support OC12 throughput.

To improve regional and Internet connectivity needed to support research collaborations, PNNL increased the Internet connection to the Pacific Northwest Gigapop (PNWGP) from OC3 to OC12. PNNL joined the Internet 2 consortium and the Abilene network and utilized the PNWGP connection to provide OC12 connectivity to research partners other than those directly connected to ESnet.

PNNL also established a $1.0 \mathrm{Gbps}$ connection to and peering at the Three Rivers Internet Exchange (TRLINX) in APEL to improve local area connectivity and performance. This connection has also been used to implement a $10 \mathrm{Mbps}$ backup Internet service provided by Charter Communications.

\section{Classified Computing}

Outstanding: 4 of 5 milestones met. PNNL provided network access to one high performance classified computing resources to address the local need driven by very large and complex classified models and analyses. A 64node high-performance clustered computer system, operational September 26, 2003, was installed in the 3760 building to provide local classified computing capability.

\section{Area Infrastructure Study}

Outstanding: 5 of 5 milestones met. The PNNL Director chartered an internal task force to formulate a strategy for transitioning the Laboratory's research and development activities from legacy facilities in the 300 Area. The core elements of that strategy will be 1) obtaining joint concurrence from the DOE-EM (RL) and DOE-SC (site office) on a schedule, and 2) as part of that schedule, obtaining approval by DOE-EM and DOE-SC for the DOE-RL recommended operating transition period for the Radiochemical Processing Laboratory (the 325 Building). During FY2003, PNNL invested more than $\$ 1.2 \mathrm{M}$ in overhead funding to refurbish several laboratories within the RPL facility and to consolidate staff and research capabilities from the 3720 facility.

The 300 Area contains approximately 700,000 square feet of PNNL facilities, or 35 percent of the total. An engineering study of utility infrastructure requirements necessary to support the 300 Area transition strategies has been completed and will support PNNL's overall strategy of transitioning PNNL's 300 Area capabilities to a modern research campus, as follows:

- Consolidate laboratory operations to allow divestiture from facilities targeted for deactivation,

- Maintain operations of the 318 and 325 Buildings until their mission need is completed, 
- Construct new utility infrastructure and roads to permit the relocation of laboratory capabilities from the 300 Area, and

- Build new facilities to support the relocation of both R\&D and support staff. The study produced site utility drawings including various options for the provision of utilities, including cost estimates. The study, completed ahead of schedule, was a key deliverable in support of the 300 Area Task Force recommendations.

\section{Abilene Network 10-Gbps Optical Upgrade - 2002-2003}

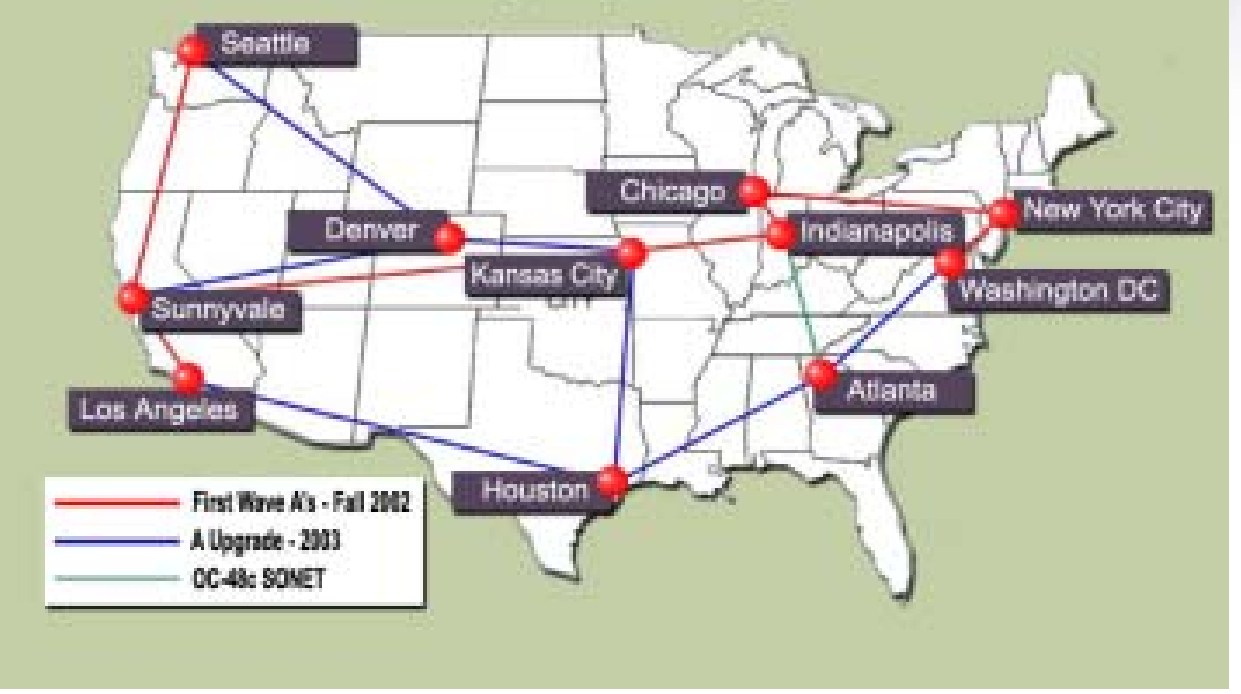

Figure 2.2.4a. PNNL connects to the Abilene Network via OC12 to the Pacific Northwest Gigapop in Seattle. The Laboratory connects to over 200 major research universities nation-wide via Abilene, which has a 10-Gbps backbone.

\section{Pacific Northwest Naticnal Laboratory 300 Area Transition \\ Utility Infrastructure Study}

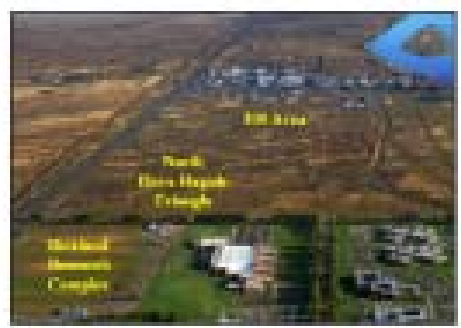

July 2003

Vaake nertimost

Witicex Lborating

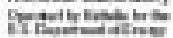

Corcant

Kontwat in

Figure 2.2.4b. PNNL evaluated utility infrastructure requirements for the future. 


\subsection{1a Commitments to Improve the Effectiveness and Efficiency of Integrated ES\&H Management Systems}

\section{The successful completion of all agreed-upon FY2003 ES\&H Management System improvement milestones, plus additional improvements, continues to demonstrate PNNL's long-term commitment to making our set of integrated ES\&H systems, processes, and tools measurably better.}

\section{What We Agreed To...}

- We developed and deployed the new Electronic Prep \& Risk (EPR) system. This new system integrated and enhanced existing hazard analysis tools to better assure the consistent and effective identification, evaluation, and mitigation of hazards; and includes an efficient work authorization process.

- We rolled out Integrated Operations System (IOPS) concepts and tools to the Marine Science Laboratory in Sequim, thus completing a multi-year effort to deploy IOPS to all PNNL's lab-intensive facilities. IOPS is the bench-level process PNNL established to communicate safe laboratory practices, identify and control workspace hazards, identify and obtain appropriate training, and authorize access to workspaces for an efficient and productive laboratory. With implementation across the Laboratory complete, the major components of IOPS are working. The IOPS approach, supported by management and staff, has achieved success due, in large part, to engaged staff and management working effectively with the hardware and software components. The system facilitates safe research by efficiently identifying and managing ES\&H risks, and then implementing mitigating actions commensurate with those risks (graded approach). This approach maximizes the time researchers have to devote to research. Staff and managers who have come to the Laboratory from other research environments say IOPS is the best way they've seen to manage safety.

- We enhanced the Radiological Material Tracking System to provide integration of requirements so key stakeholders can now perform real-time checking to ensure compliance with Facility Use Agreement (FUA) operating boundaries for radioactive materials. Currently, this system is in limited use in key radiological areas.

\section{....And More}

PNNL is committed to enabling science and research through the integration of ESH\&Q systems and tools. We continued to enhance our systems and tools this year to make science and research safer/more efficient; here are some examples:

- In November 2002, we formally obtained ISO 14001 registration, providing third-party confirmation of the maturity of our environmental management system.

- Fully implemented WISHA Ergonomics Rule and developed web-based training for staff. This included completing workspace evaluations for majority of employees found to be in "Caution Zone Jobs" and developing an instruction manual that H\&S representatives use when conducting ergonomic assessments.

- Developed and implemented system controls for working with biological materials and developed web-based training for staff working with select agents.

- Developed a website dedicated to beryllium in order to enhance worker communication and aid work planning.

- Identified a dedicated S\&H representative to manage contractor construction safety and develop administrative protocols/procedures.

- Developed and implemented an in-vivo bioassay waiver program to aid radiological monitoring of staff.

- To enable users of the Standards-Based Management System to find information more quickly, we expanded our keyword index capabilities.

- Completed and implemented Re-Engineering of the Radioactive Waste Operations program, which will make processing radioactive waste more efficient and compliant. 
Concurrent with these significant programmatic improvements, ESH\&Q has continually reduced the costs of its management systems core services. The cost of ESH\&Q core services has decreased by $21 \%$ as of the end of FY2002 from FY1999 baseline costs. By the end of
FY2004, we project our core services will cost $71 \%$ of our FY1999 baseline. These numbers are all the more impressive given that we have absorbed nearly $\$ 1.3 \mathrm{M}$ in new scope changes over the past five years.

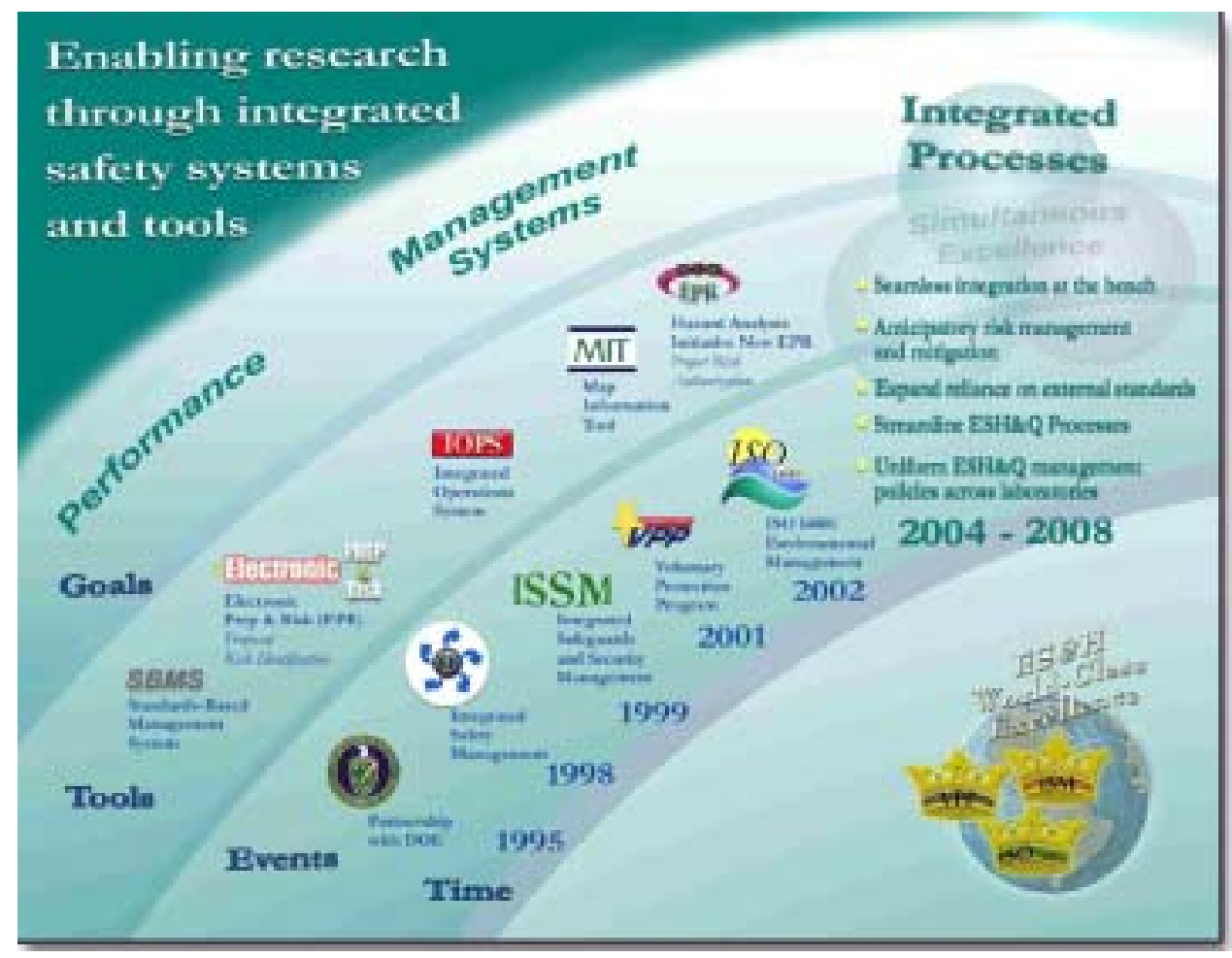

Figure 2.3.1a. PNNL continues to strive for seamless integration of ESH\&Q processes to enable science while keeping staff safe. 


\subsection{1b Commitments to Improve the Effectiveness and Efficiency of Business-Related Management Systems}

\section{By fulfilling DOE expectations-and more-in FY2003, we continue to develop innovative approaches to Laboratory business operations that enable outstanding research, maximize value per research dollar, maintain forefront facilities and equipment, and protect DOE's information assets.}

\section{What we agreed to...}

- We developed and implemented a new Proposal Pricing System, which streamlined the process and tools associated with proposal pricing. The new system provides a central repository for out-year and escalation rates, as well as proposal pricing data, allowing tracking of resource commitments and dollar value of proposals. Project resources are priced using the same accounting tables and algorithms used by the Battelle cost processor, thus ensuring extensibility, consistency, and accuracy, and eliminating our project management support staff's need to maintain a duplicate pricing engine and forward pricing rates.

- We implemented an 8-point action plan in FY2003 to strengthen management controls around work authorization and funds control. This action significantly improves documentation, controls, and communication, resulting in a heightened awareness of work authorization requirements on the parts both DOE field and HQ staff and PNNL staff; and the need to anticipate and resolve work authorization issues in a timely manner. DOE 1830 overruns in general have been kept well under control and within established performance metrics. In addition, relationships and reporting mechanisms have been solidified with DOE PNSO (RL/AMT) to build trust and confidence in our systems. This trust will be important as we move forward towards full transition to reporting to Office of Science. The action plan included completion of:

1. A root cause analysis,

2. Documentation and clarification of PNNL's work authorization process,

3. A revised record of decision (ROD) for DOE Order 412.1,
4. Reorganization of the financial organization responsible for work authorization and funds control for increased focus,

5. Updating of the relevant policies,

6. Reviewing the complete set of financial systems for other possible informal approvals being used to drive policy,

7. Re-evaluation of the set of financial RODs for accuracy and completeness, and

8. Assessing the appropriateness of maintaining the Work for Others and User Permit Anticipatory account policy.

- We developed and submitted to DOE a document that framed the minimum elements that a contract clause regarding Minimum Performance Standards (MPS) would have to contain or embody before Battelle would enter into negotiations to replace the DEAR 970.52153 Conditional Payment of Fee/PEFA clauses/language with an MPS clause. The negotiation of the elements of such a clause was not included in the preparation of the new contract signed in FY2003.

- In response to the recommendations from the FY2002 study on the Customer Service Model (CSM), we initiated a process to update governance expectations for CSM managers. The objectives of this effort were to identify and resolve implementation issues, update roles and responsibilities, and update performance measures. The CSM performance measures were to be revised in close collaboration with the broader effort to develop a Laboratory dashboard, which was delayed until FY2004. As a result, it was not possible to select the CSM measures suitable for the Laboratory dashboard this year, and we did not meet our 
commitment to do so. However, significant progress was made on the CSM update effort. Meetings were held with most CSM managers to obtain their input. Their contributions on product line issues, responsibilities, and metrics were compiled and presented to the Research Council, who concurred with the recommended changes and future actions. The Research Council directed that the inputs from the sector, product line, and capability managers should be consolidated and brought back to them for final approval. In first quarter FY2004, an integrated set of responsibilities and performance metrics for all CSM processes will be presented to the Research Council. The agreed upon set will be implemented and will serve as input to the Laboratory dashboard effort, which will be completed by the end of FY2004.

\section{... And more}

We initiated a Vendor Management Program, which significantly enhances how the Laboratory obtains low- dollar value purchases. We will evaluate the P-Card purchases to determine what we buy and from whom and then negotiate priced master agreements and create a web-based catalog ordering system for the Laboratory. We have also been successful in convincing large businesses to team with small businesses, which will help the Laboratory meet the goals in its Small Business Subcontracting Plan.

We have successfully implemented an action plan designed to enhance the Laboratory's P-Card. Major areas of this plan included

- Development of a new web-based training program and requiring all $\mathrm{P}$-card holders and Approving Officials to complete the training.

- A22\% reduction of P-Card holders in the Laboratory.

- Successfully obtaining a ratio of P-card holders to Approving Officials of five to one.

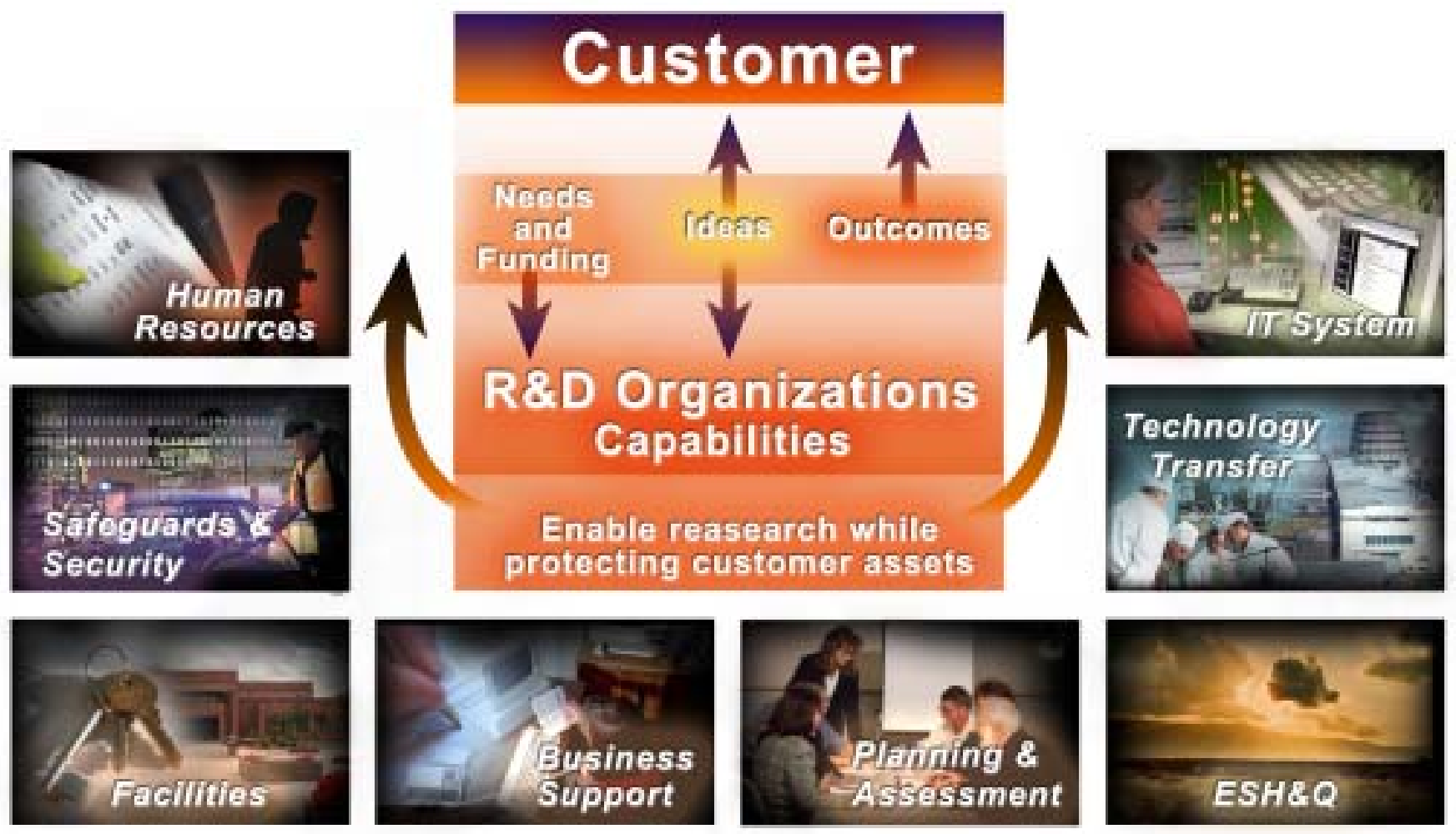

Figure 2.3.1b. This figure identifies the major elements of the integrated management approach that we have implemented at PNNL. 



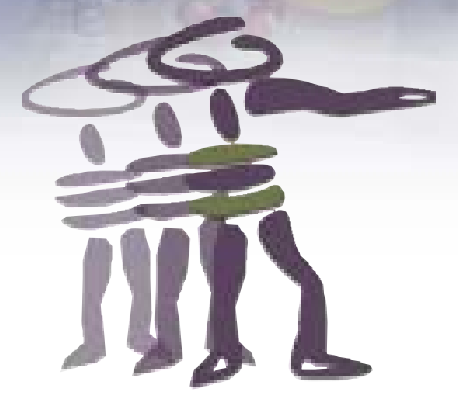

\section{Part I \\ Status of Performance Against Critical Outcomes 3.0 Leadership Excellence}

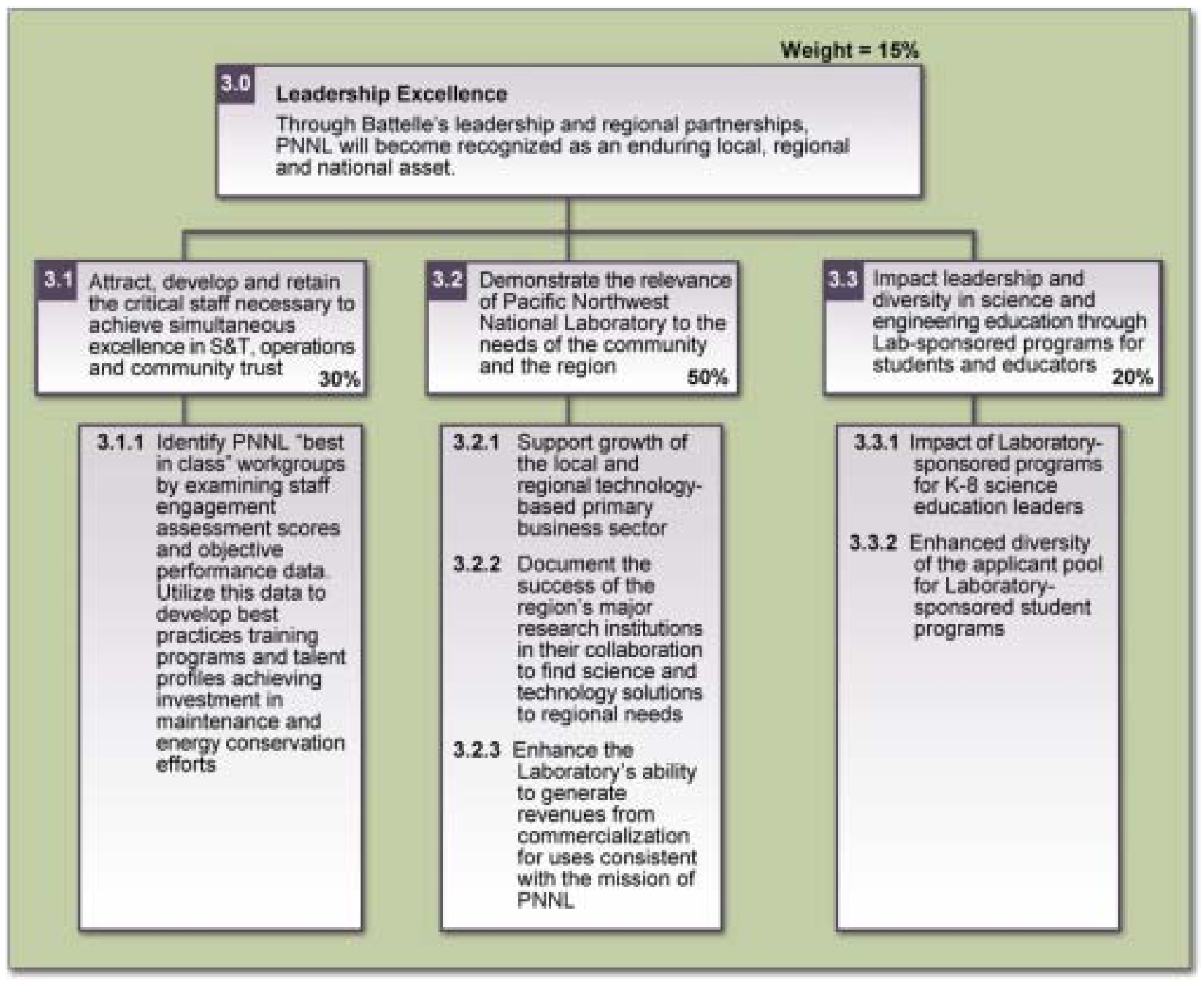




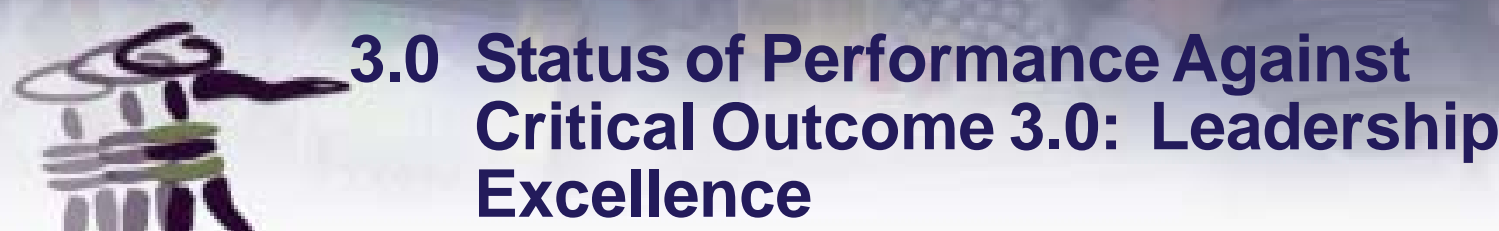

PNNL has become recognized as a local, regional, and national asset. In FY2003, PNNL met or exceeded all but one of DOE's expectations in Leadership Excellence.

This section of the report details PNNL's self-evaluation of the adjectives and corresponding value points that lead to PNNL's Critical Outcome 3.0 FY2003 score of 3.70 and performance rating of Outstanding for Leadership Excellence. Appendix A shows the adjectival and value ratings for FY2003 Critical Outcome 3.0 and all of its objectives and performance indicators (Tables A.15 - A.17).

\section{Highlights of the Three Performance Objectives}

\subsection{Attract, Develop, and Retain the Critical Staff Necessary to Achieve Simultaneous Excellence in S\&T, Operations, and Community Trust}

$\checkmark$ 3.1.1 Goal 1: Complete 50 StrengthsFinder assessments for Technical Group Managers (TGMs). Result: PNNL conducted 92 such assessments.

$\checkmark$ 3.1.1 Goal 2: Complete an analysis to define talent profiles for TGMs. Result: PNNL hired Gallup to conduct focus groups based on the first six of Gallup's Q12 characteristics of "Best Practices Managers."

$\checkmark$ 3.1.1 Goal 3: Define productivity measures for Research Division workgroups. Result: PNNL enlisted our Council of Fellows, which identified 12 productivity-defining characteristics for workgroups.

冈 3.1.1 Goal 4: Increase the overall Laboratory workplace engagement assessment GrandMean score by .09. Result: PNNL achieved two-thirds of this stretch goal, increasing the score by .06 .

\subsection{Demonstrate the Relevance of Pacific Northwest National Laboratory to the Needs of the Community and the Region}

$\checkmark$ 3.2.1.1 Goal: Start or expand a total of six or more businesses in the area where Battelle had a material role in their establishment. Result: PNNL achieved the goal of six.
3.2.1.2 Goal: Provide technical assistance to at least 40 firms, with $85 \%$ of them reporting "very satisfied." Result: PNNL assisted 46 firms and achieved a satisfaction rating of $100 \%$.

$\checkmark$ 3.2.1.3 Goal: Develop and champion at least one new economic development initiative to help grow and diversify the local and regional technology sector. Result: PNNL developed and implemented five such initiatives.

\ 3.2.2.1 Goal: Identify and describe how two technologies and/or capabilities from northwest institutions might address one or more regional needs. Result: PNNL focused on water resource management needs and identified 10 new capabilities.

$\checkmark$ 3.2.2.2 Goal: Develop a mechanism for commercializing an applicable technology or capability. Result: PNNL worked with students from the University of Oregon to develop a prize-winning business plan based on a water treatment technology.

$\checkmark$ 3.2.2.3 Goal: Formally present the Linking Regional Resources program and accomplishments to one governmental and one non-governmental agency in one or more states of the region. Result: PNNL made presentations to two governmental and two non-governmental organizations.

$\checkmark$ 3.2.3 Goal: Generate at least $\$ 900,000$ in licensing revenue from DOE-derived inventions. Result: PNNL generated $\$ 1,326,746$ in such revenue, exceeding the mark by $47 \%$.

\subsection{Impact Leadership and Diversity in Science and Engineering Education through Lab-Sponsored Programs for Students and Educators}

$\checkmark$ 3.3.1 Goal: Receive an evaluation of 10 or higher out of 12 from $75 \%$ of LASER leadership teams surveyed about the impact of the program. Result: PNNL received the desired ratings from $93 \%$ of those surveyed. 
3.3.2 Goal: Achieve a 50\% increase in the diversity of the pool of applicants for PNNL project-funded fellowships. Result: PNNL far exceeded its outstanding target, achieving an increase of $76 \%$.

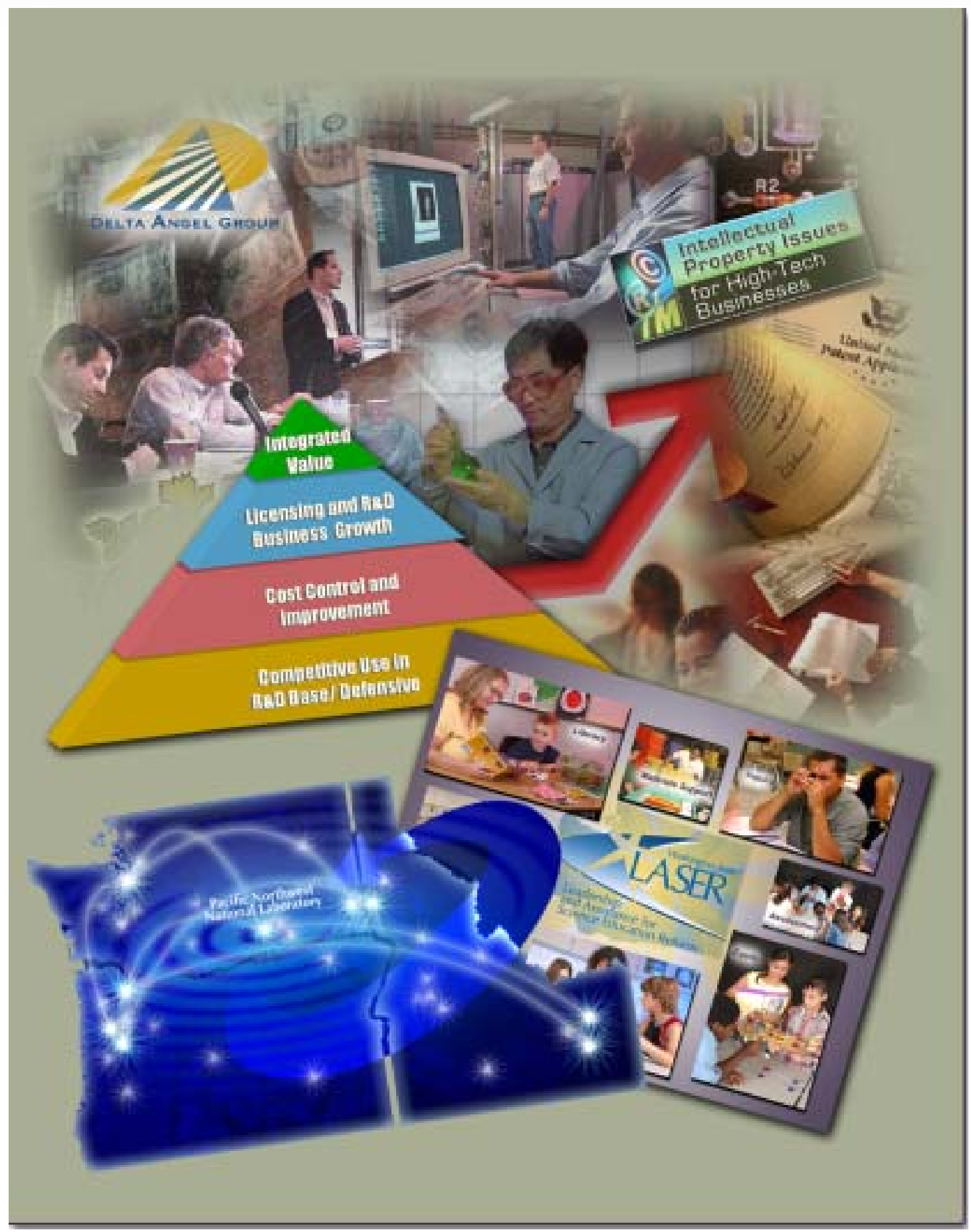

Figure 3.0. PNNL is recognized as a local, regional, and national asset as evidenced by its role in growing and diversifying the local and regional economy, linking regional resources, and science and engineering education; and its ability to generate revenues from commercialization. 


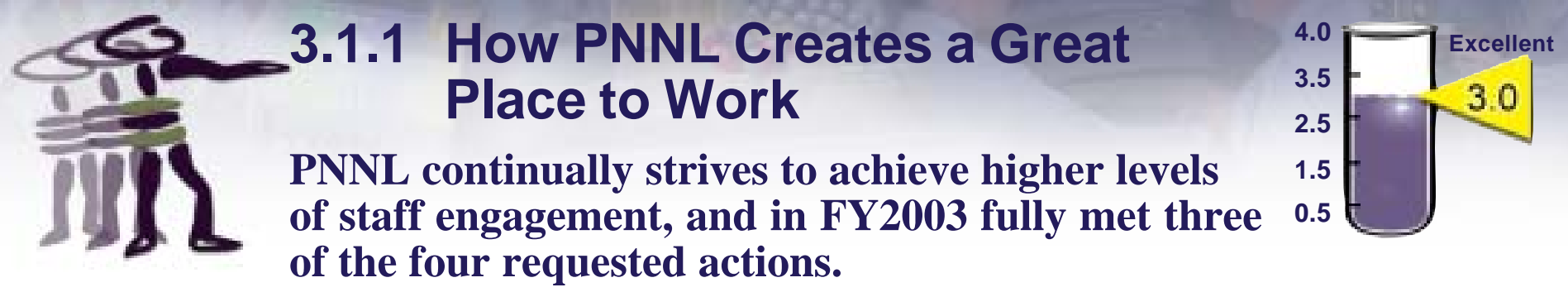

\section{Actions 1 and 2: What Makes Great Managers?}

In FY2003, we asked 75 managers and 17 PNNL executives to take the Gallup StrengthsFinder ${ }^{\mathrm{TM}}$ assessment to examine what signature strengths were prevalent among highly effective managers and executives and to help us define talent profiles for our Technical Group Managers. All 17 PNNL executives and 43 of the 75 managers took part in a further study to examine what signature strengths were prevalent in each group of highly effective managers and what differences were evident between effective managers at the mid-level of the organization versus the executive level.

The most common strengths of these two groups were Maximizer, Arranger, Relator, and Achiever. With the Maximizer strength, what both groups of managers have in common is a desire for excellence; they seek a certain level of expertise to take something good and turn it into something great. They are at home in dynamic and interactive environments, and their Arranger strength assists them in figuring out how all the pieces and resources can be arranged for maximum productivity. The Relator strength illustrates an inclination to work hard with friends to achieve a goal. These managers are less interested in "political" considerations. They will use their informal network to get things done. Achievers tend to be busy and productive; they have a great deal of stamina and take great satisfaction from working hard.

In our analyses, we identified some distinct strengths in our managers and executives. Our managers were more likely than their executive counterparts to manifest these strengths: Harmony, Achiever, Command, Connectedness, Ideation, and Learner. The single most common strength of PNNL managers was Achiever. Conversely, our executives were more likely to demonstrate these strengths: Individualization, Relator, Focus, Competition, Maximizer, Futuristic, Input, and Intellection. The two most common strengths of PNNL executives were Relator and Maximizer.
Also during FY2003, we conducted focus groups to define talent profiles for our Technical Group Managers. As a basis for strengths-based selection, Gallup is a proponent of studying an organization's best performers in each key role. One way they accomplish this is to conduct a simple focus group during which they ask a series of open-ended questions to get a feel for the role. These focus group interviews investigated what the "Best Practices Managers" do in their workplace that differentiates them from other managers. To make the sessions manageable in the time allotted, we focused on the first six Q12 questions. Of the six Q12 questions, questions 1, 3 and 5 were examined more closely due to their strong correlation to productivity. Results of these interviews indicate that great managers at PNNL share robust talent profiles.

Under Question 1 - I know what is expected of me at work, managers clearly define and communicate expectations, keep associates informed of decisions that impact their work, frequently interact with their staff, and speak with a consistent voice as a management team.

Under Question 3 - At work, I have the opportunity to do what I do best every day, managers identify and use the strengths of their employees, challenge employees to find out what they do best, encourage employees to honestly express their professional needs and goals, help employees devise effective road maps to do a better job, minimize their focus on correcting their employees' weaknesses, and help employees find their best fit by maximizing their strengths.

Under Question 5 - My supervisor, or someone at work, seems to care about me as a person, managers trust their employees, are always fair by their employees, take an active interest in the professional and personal achievements of their employees, and realize they receive big dividends if they take the time to care.

The best managers are those who help their staff members take ownership of their workgroup data and make their staff part of the solution. These managers lead 
with powerful examples and practice what they preach. They are engaged and strive to engage their people.

\section{Action 3: How do we Know We're Productive?}

To define research productivity at PNNL, and to establish some common measures and metrics to identify highly productive workgroups, we enlisted the help of our Council of Fellows. This extraordinary group is comprised of staff members who have endured a rigorous Laboratory-wide nomination and review process and have attained the highest level of scientific and research excellence within the Lab. Our Fellows identified 12 characteristics that define highly productive research groups at PNNL. We have begun to correlate these characteristics to highly engaged workgroups.

\section{Action 4: Are We More Engaged?}

Our overall workplace engagement assessment GrandMean score is 3.81, which is up from 3.75 over the previous year. (To fully meet the goal for this action, our score would have to have been 3.84.) The Gallup $75^{\text {th }}$ percentile (best in class) score is 3.96. Our score indicates that we are making steady progress toward achieving our goal of being a best in class employer.

\section{What Now?}

To move from above average to best in class we will continue to provide varied career path options and help staff identify their strengths and then team them with great managers, thereby creating an engaging work environment.

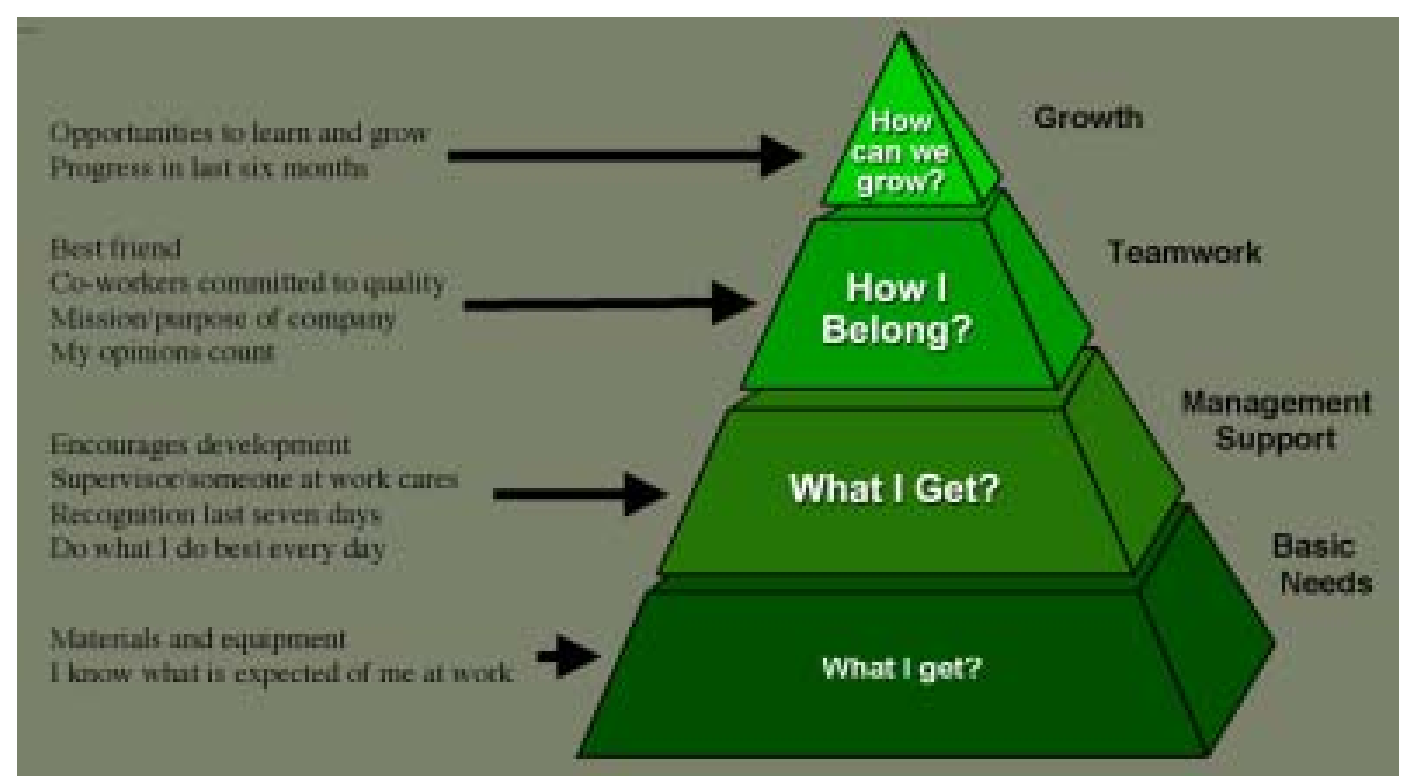

Figure 3.1.1. PNNL measures staff engagement according to these 12 characteristics of an effective workplace. 


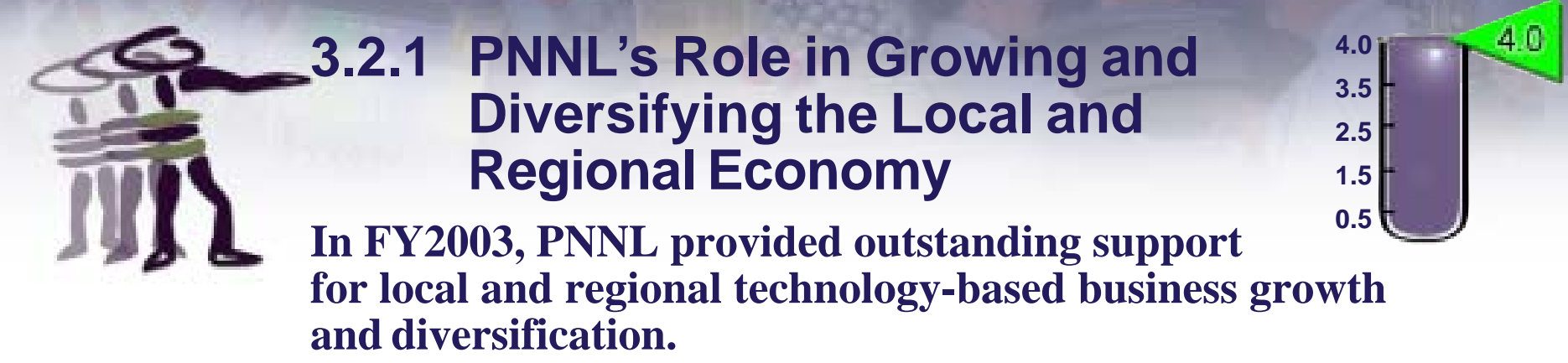

For the eighth consecutive year, PNNL achieved outstanding performance in its economic development programs. With substantial support from PNNL, six businesses started or expanded offices in the Tri-Cities. Lab researchers provided no-cost technology assistance to 46 firms in the Northwest region, providing specialized expertise not readily found in the private sector. PNNL implemented five new initiatives to advance the local and regional tech sector.

\section{Establishment of New Businesses and Expansions in Local Area}

In FY2003, PNNL technology, technological assistance, and entrepreneurial support played a significant part in establishing or expanding of six local businesses: WebOps, LLC, a Web-based information management provider; TrueActive Software, a developer of software that monitors computer activity for businesses, government, and consumers; Safe-View, Inc., a company that is commercializing a security-related imaging technology developed at PNNL; Vista Engineering Technologies, LLC, a provider of engineering and technology development services for government and industry; Caldus Semiconductor, Inc., a developer of semiconductors that operate at extremely high temperatures; and Essential Solutions, creator of KidCompass, a software package that tracks results of student reading assessments and provides research-based instructional strategies for individual reading improvement.

\section{Technical Assistance to Local and Regional Firms}

In FY2003, PNNL's technology assistance to 46 firms in the Pacific Northwest earned a $100 \%$ satisfaction rating from the recipients. Under the Technology Assistance Program, PNNL engineers and scientists offer up to a week of technology assistance annually at no cost to qualifying commercial businesses. Business owners who received PNNL's technology assistance said that it helped them develop and improve products, solve technical problems, ensure quality, and save time and money.

\section{New Economic Development Initiatives}

In FY2003, PNNL developed and implemented five initiatives to help grow and diversify the local and regional technology sector: 1 and 2) two educational seminars for entrepreneurs where experienced Northwest tech business experts shared information and recommendations with entrepreneurs at two public events on the topics of intellectual property, seeking investment capital, building experienced management teams, creating brand awareness, and corporate structure; 3 ) a recruiting assessment study to assess the feasibility of attracting targeted technology firms to take advantage of PNNL's technology expertise, land holdings, and user facilities; 4) sponsorship and board membership of the Delta Angel Group of Spokane, which helps link inland Northwest tech entrepreneurs to investors; and 5) establishment of the first satellite downlink location in Southeastern Washington State for the MIT Enterprise Forum broadcasts, at WSUTC. MIT Enterprise Forum, Inc., an organization of alumni of the Massachusetts Institute of Technology, offers satellite broadcasts to educate entrepreneurs and foster business networking.

The outcomes of Initiatives 1, 2, and 5 are an increasingly informed local population of tech entrepreneurs and stakeholders with more connections to experienced business professionals who can help them succeed and avoid pitfalls. Also, one of the firms that attended the seminars realized that it needed to hire a new outreach firm and a new CEO, both of which it has since done, resulting in substantial growth. So much so, that the firm was also counted in 3.2.1.1. Initiative 3 , the recruiting assessment, will be used as part of a broader decision process about attracting tech firms as PNNL's neighbors, which 
will in turn become part of PNNL's emerging strategy to develop a R\&D park around the Lab. The recruiting assessment identified one industrial sector, electronics, that should be of particular interest as a source of prospective neighbors. As a result of Initiative 4, Delta Angel Group sponsorship, two local tech firms were invited to present to the group for equity investment consideration, with other local firms in the Delta pipeline. An EDO staff member was also invited to join the Delta board, further entrenching Tri-City deal flow into the group. The potential impact of Delta is a nearby source of equity investment for Tri-City firms.

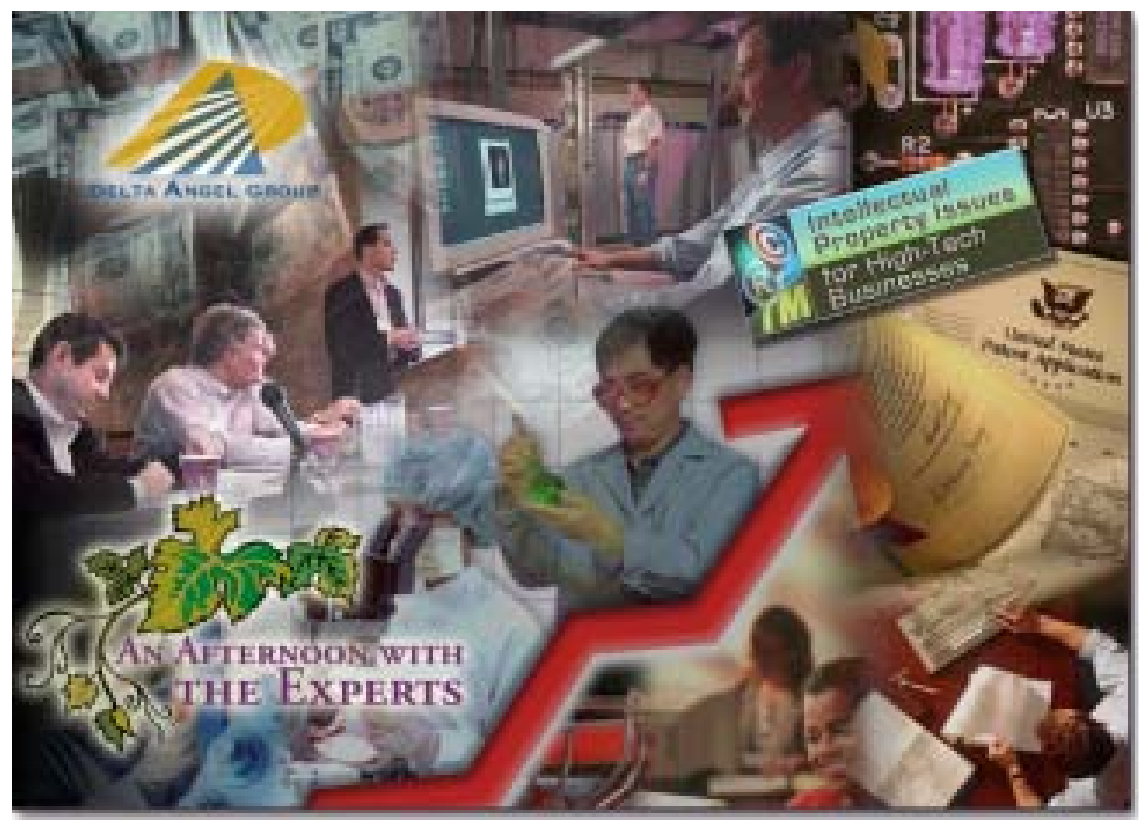

Figure 3.2.1a. PNNL helped start and expand high-tech businesses, as well as connecting entrepreneurs with experienced tech business experts and investors.

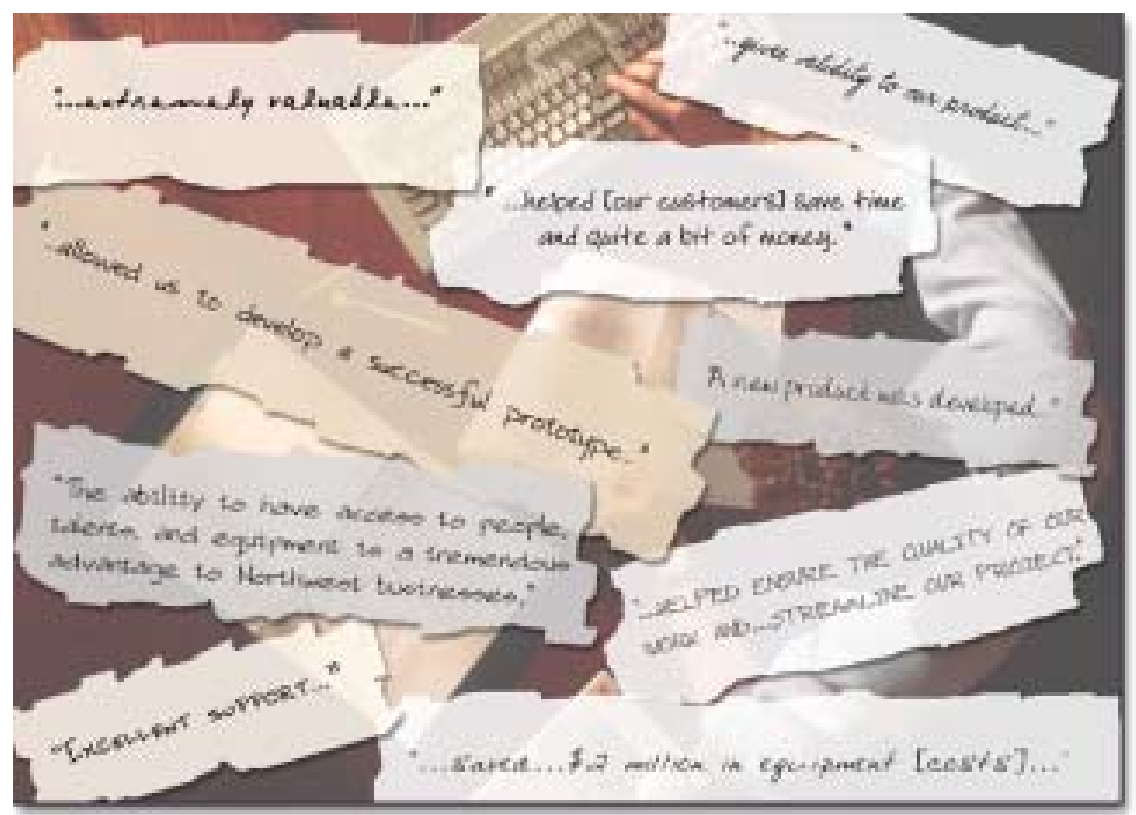

Figure 3.2.1b. Quotes from business owners who received PNNL technology and entrepreneurial assistance in FY2003 underscore how highly the commercial sector values this expertise. 

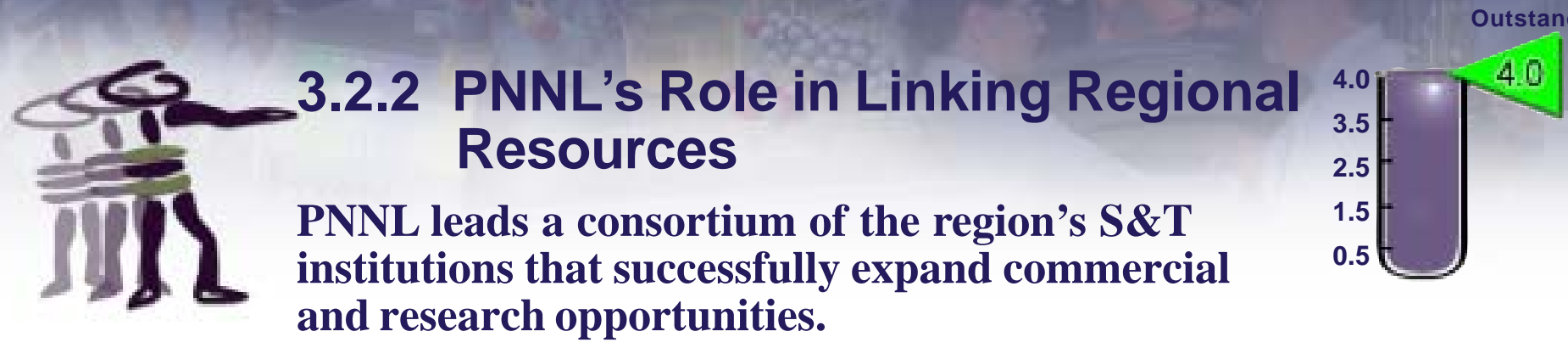

\section{Solutions to Regional Needs}

In FY2003, the Linking Regional Resources (LRR) Steering Committee elected to focus on finding solutions to the region's water resource management needs from among the S\&T resources of its member institutions. PNNL exploited the LRR patent and technical literature inventories contained in Starlight to uncover water resource technologies and/or research capabilities that might represent solutions, and teamed with the Pacific Northwest Regional Collaboratory (PNWRC) to identify the needs. The PNWRC is a multi-institutional partnership housed at PNNL, whose mission is to facilitate the integration of geospatial technologies and data to serve the needs of resource managers and decision-makers across the Pacific Northwest. Using Starlight, 10 new capabilities from University of Washington and Oregon State University were identified for the PNWRC. The identified capabilities specifically augment remote sensing techniques as applied to water resource management needs. Another 30 capability areas were identified that may have a technical and/or programmatic role for the PNWRC. The PNWRC plans to augment future project scoping relative to new capability findings.

\section{Applicability of S\&T Solutions}

The application of a water treatment technology to meet a regional need was the subject of a prize-winning business plan. Students from the University of Oregon worked with PNNL to develop a business plan based on a water treatment technology that was identified as a possible solution to drinking water issues in the northwest. The technology, Self-Assembled Monolayers on Mesoporous Supports (SAMMS), was developed at PNNL and could be modified to meet the requirements associated with treating drinking water. The business plan outlines a strategy for taking this technology to the market. As a testament to the quality of the business plan, it won 4th place in a national competition (UT Austin's Moot Corp.) and received broad national exposure. PNNL is currently looking at the applicability and market need associated with point-of-use application for this technology.

\section{Relationships Among Research Northwest Institutions}

The work of the LRR consortium has been published in multiple venues. Presentations reviewing the Linking Regional Resources program and its vision of bundling intellectual property from Northwest research institutions were given at the following events: BIO 2003, The Washington State Senate Technology and Communications Committee Hearing on Technology Transfer, The Northwest Entrepreneurial Network - Biotechnology Meeting, and the Federal Lab Consortium Annual Meeting TechEntreprise 2003. In all cases, the audiences were interested in the concept and exploring how it might be applied to areas of interest to them. 


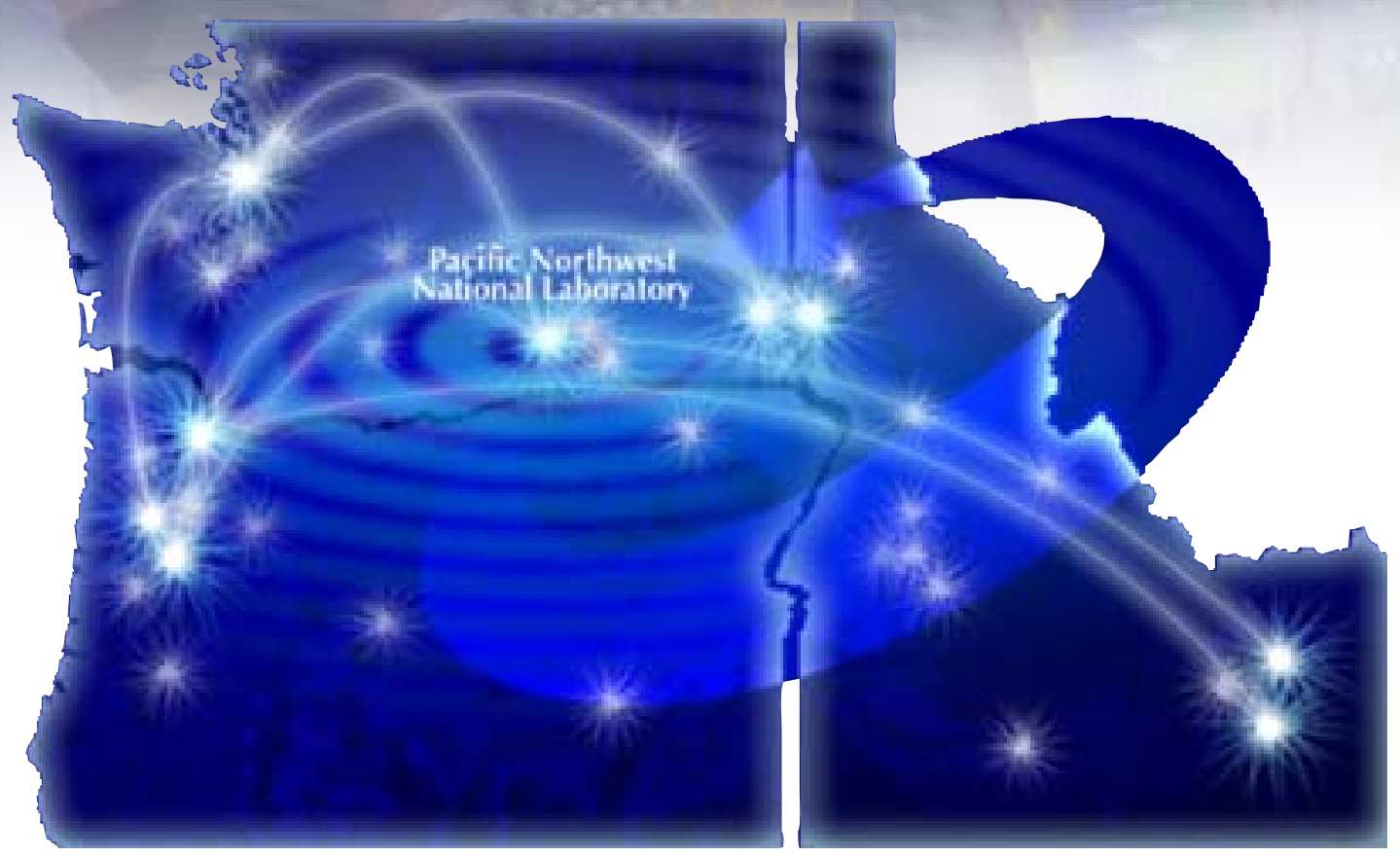

Figure 3.2.2. Using its Starlight system, PNNL links S\&T institutions and their resources. 


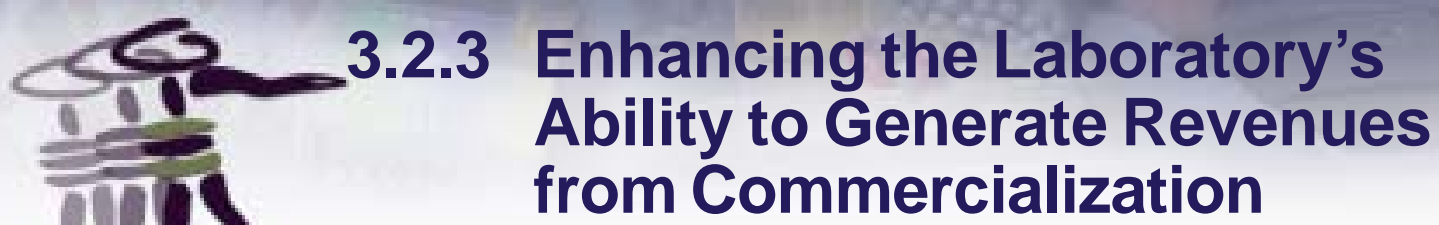

Outstanding

\begin{abstract}
DOE-derived inventions substantially exceeded the requirements for an "outstanding" rating, and resulted
\end{abstract} \\ As a result of strategic intellectual property
management and aggressive technology development
and licensing, PNNL's FY2003 licensing revenues from \\ As a result of strategic intellectual property
management and aggressive technology development
and licensing, PNNL's FY2003 licensing revenues from \\ As a result of strategic intellectual property
management and aggressive technology development
and licensing, PNNL's FY2003 licensing revenues from in increased funds for Laboratory investment.}

Enhancing PNNL's ability to generate revenues from commercialization is an important objective for reaching our goal of investing returns from commercialization into laboratory staff and facilities. In FY2003, PNNL generated $\$ 1,326,746$ in revenues derived from DOE inventions-over $47 \%$ more than needed for a rating of outstanding. In addition, other value beyond revenue was generated in "returns" to PNNL.

To enhance the value generated from IP, during FY2003, we made several changes in the assignment and management of PNNL's intellectual property portfolio. Commercialization managers were aligned with Laboratory product lines to gain benefit from and complement R\&D programs for delivering the Laboratory's technology. This alignment with product line management resulted in increased mutual benefit in technology marketing, whether intellectual property commercialization or R\&D program. It also allowed for more effective leveraging of business development resources.

The intellectual property was organized by crosscutting technology portfolios, which were then assigned to a specific Commercialization Manager, who provides domain expertise. Working with the technical portfolio innovators, Commercialization Managers were able to better understand programs and capabilities associated with inventions generated at PNNL. Intellectual property development, evaluation, communication, and decisionmaking processes were improved to increase the quality of technical, IP, business and market assessment, resulting in improved investment decisions. Improvements were made in cycle time for evaluating and dispositioning newly generated intellectual property. The average number of days to review and make a decision regarding each new invention in FY2003 was improved to 75 days-a six-day improvement from FY2002.

Complementary to our enhanced intellectual property development and management activities, we made substantial efforts in a more intensive approach to licensing PNNL technologies. In particular, we made selective investments to improve the attractiveness of technologies, and to enhance techniques to publicize and promote available technologies. For example, we rebuilt the Available Technologies website, which is located on our external website. The rebuild provides for a much better interface for visitors seeking information about technologies available at the Lab, with enhanced searching capability. As well, the new website provides more salient information, resulting in an increase of our visitors by a factor of 100. Greater attention to management of existing agreements as well as to the administration of newly established agreements, helped to identify new opportunities for technology transfer.

The results of these efforts, beyond revenue generation, can also be measured in terms of the increased $R \& D$ from joint development activities, and in the value of equipment obtained for the laboratory, either through license agreements or investment of commercialization returns. Specifically, we obtained mass spectrometers through license agreements and were able to enhance our rapid manufacturing and dosimetry laboratories through investments made from commercialization returns. 


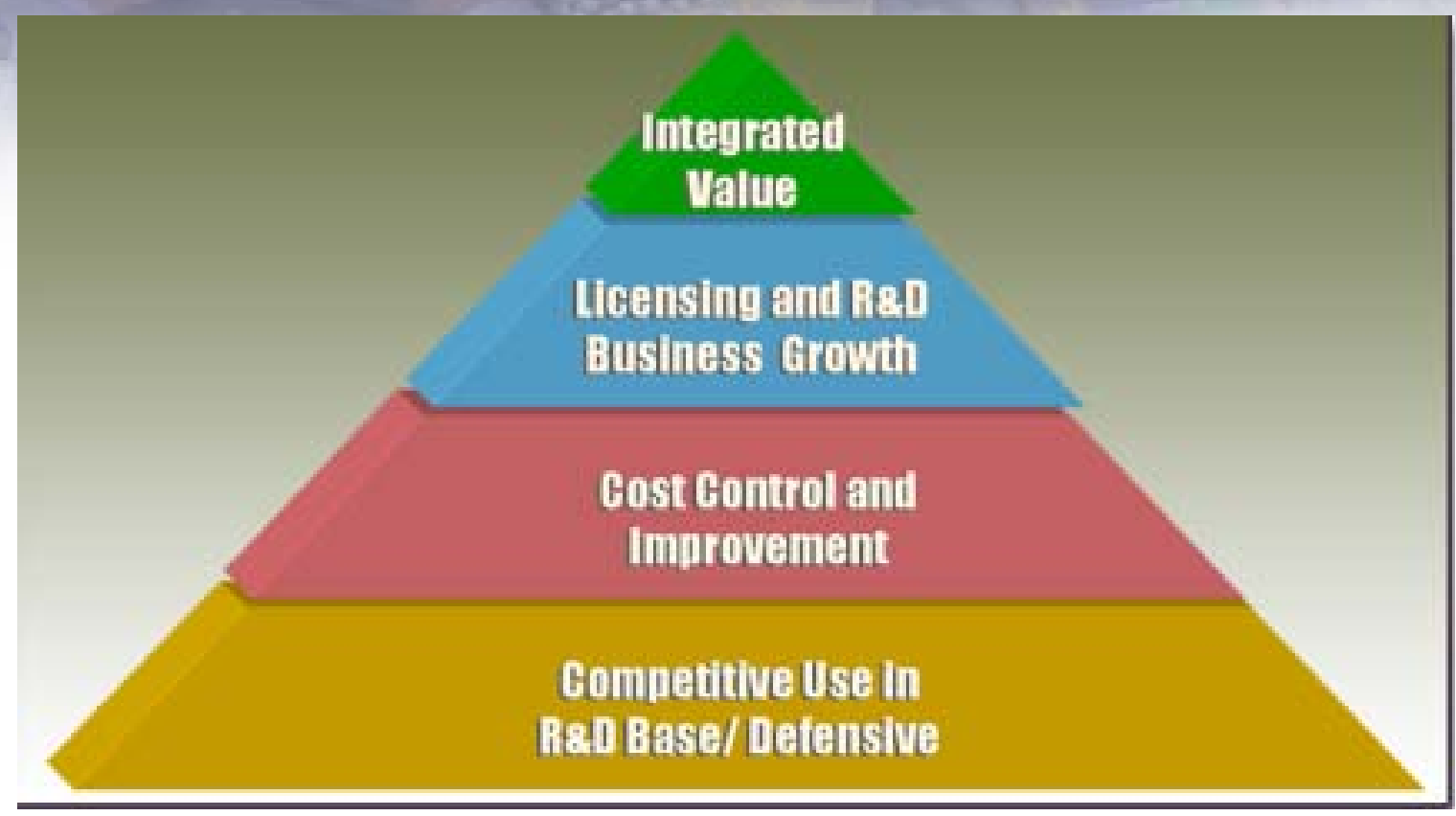

Figure 3.2.3a. This graphic illustrates the strategic approach that results in value creation through the growth of complementary R\&D and licensing programs.

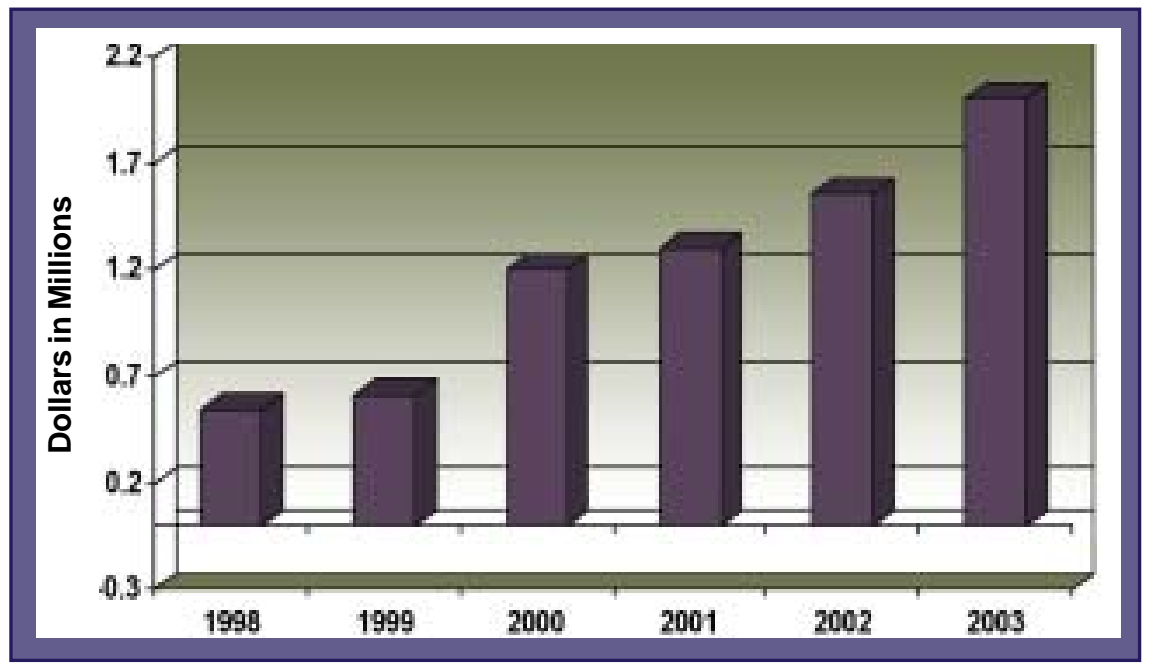

Figure 3.2.3b. Over the last three years, changes in our approach to IP portfolio management, attention to portfolio revenues and expenses, and exploitation of dual licensing and contract R\&D opportunities for product lines, have resulted in an increase in total consideration to the Laboratory in return for IP licensing. 


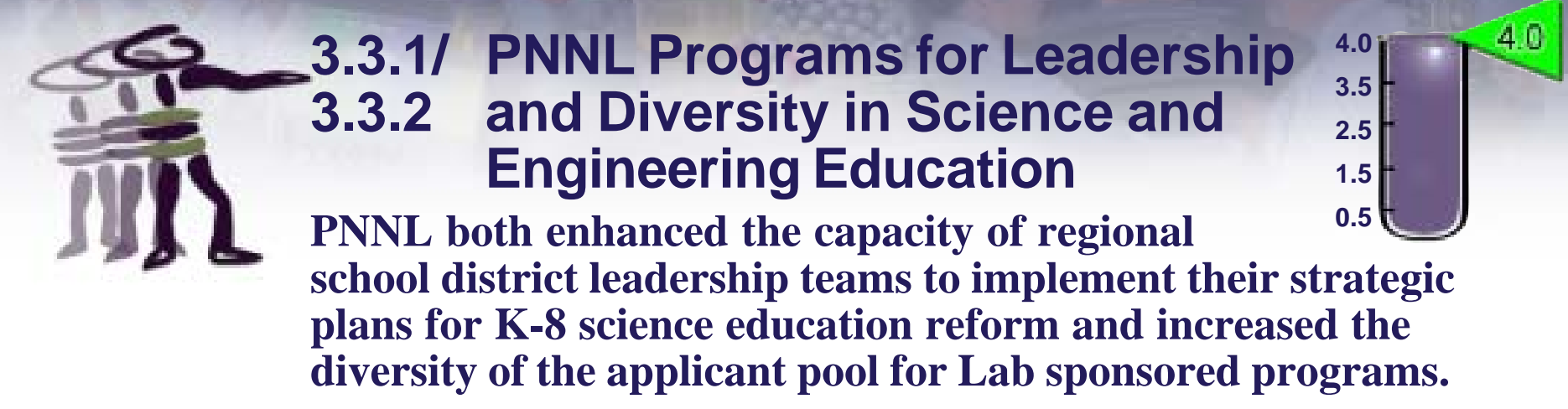

The Leadership and Assistance for Science Education Reform (LASER) Program enhanced the capacity of school district leadership teams to design and implement their strategic plans for K-8 science education reform. LASER 1) enabled leadership teams to network with other leadership teams to learn "best practices," 2) provided presentations and workshops that enhanced knowledge and skills, and 3) fostered the development of action plans for science education reform.

\section{Enhancing K-8 Science Education}

Survey results illustrate Leadership and Assistance for Science Education Reform (LASER) program effectiveness exceeding the target for an outstanding rating. Ninety-three percent of participating leadership teams gave LASER an outstanding rating for its technical assistance efforts. The threshold for outstanding is $75 \%$. This technical assistance focused on:

- Helping school districts introduce and implement a comprehensive K-8 science education program featuring a standards-based, inquiry-centered, science curriculum that met rigorous $\mathrm{R} \& \mathrm{D}$ criteria, including research in cognitive development, technical review by scientists and engineers, and widespread testing in classrooms.

- Supporting school district leaders in their efforts to raise understanding about the upcoming Washington Assessment for Student Learning for Science (WASLScience) and to implement classroom-based science assessments that prepare students for success on high-stakes tests such as the WASL.

- Deepening the understanding of school district leaders about the different stages of science professional development required for teachers (e.g., novice, competent, expert) and the variety of professional development strategies needed to improve teacher knowledge, skills, and classroom practice.
- Sharing elements of best practice for establishing, managing, and sustaining science materials centers, which provide teachers with "ready-to-teach" science modules emphasizing "minds-on, hands-on" science.

- Broadening and deepening understanding of the roles that school administrators and community members (e.g., scientists \& engineers) can and should play in effective science education programs.

\section{Increasing Diversity of Student Applicants}

Intensive in-person recruiting at universities with large populations of under-served students yielded dividends in diversity of applicants for Lab-sponsored educational fellowship programs.

- In FY2002, PNNL received 679 completed applications for educational fellowships at PNNL. Of those, 46 were from African American, Hispanic, and Native American students. In FY2003, the number rose to 846, of which 81 were from African American, Hispanic, and Native American students. This increase of $76 \%$ in the number of diverse applicants far exceeded our "outstanding" target of a 50\% increase. At the same time, the percentage of the applicant pool comprised of these underrepresented groups also increased.

- In FY2003 we focused on personal visits rather than mass mailings, and showed excellent results. We visited 16 colleges and universities. In FY2002 we had 223 applicants from those institutions, and in FY2003 we had 299, an increase of $34 \%$. If we eliminate visits to regional colleges and universities where we already have a strong recruiting presence (CBC, WSU, UW, OSU), then we had a total of 22 applications in FY2002 and a total of 103 in FY2003, or an increase of $368 \%$. For the seven universities we visited specifically to recruit under-represented students, 
we went from three applicants in FY2002 to 49 in FY2003, an impressive increase of $1533 \%$. Clearly, a large part of our success in increasing our number of diverse applicants stems from our investment in recruiting at those universities in person. Of the seven conferences we attended, three were specifically for minorities. However, we don't have a robust statistical method to track how effective those visits were in generating applicants. We are looking into ways to more effectively determine our most fruitful recruiting techniques.

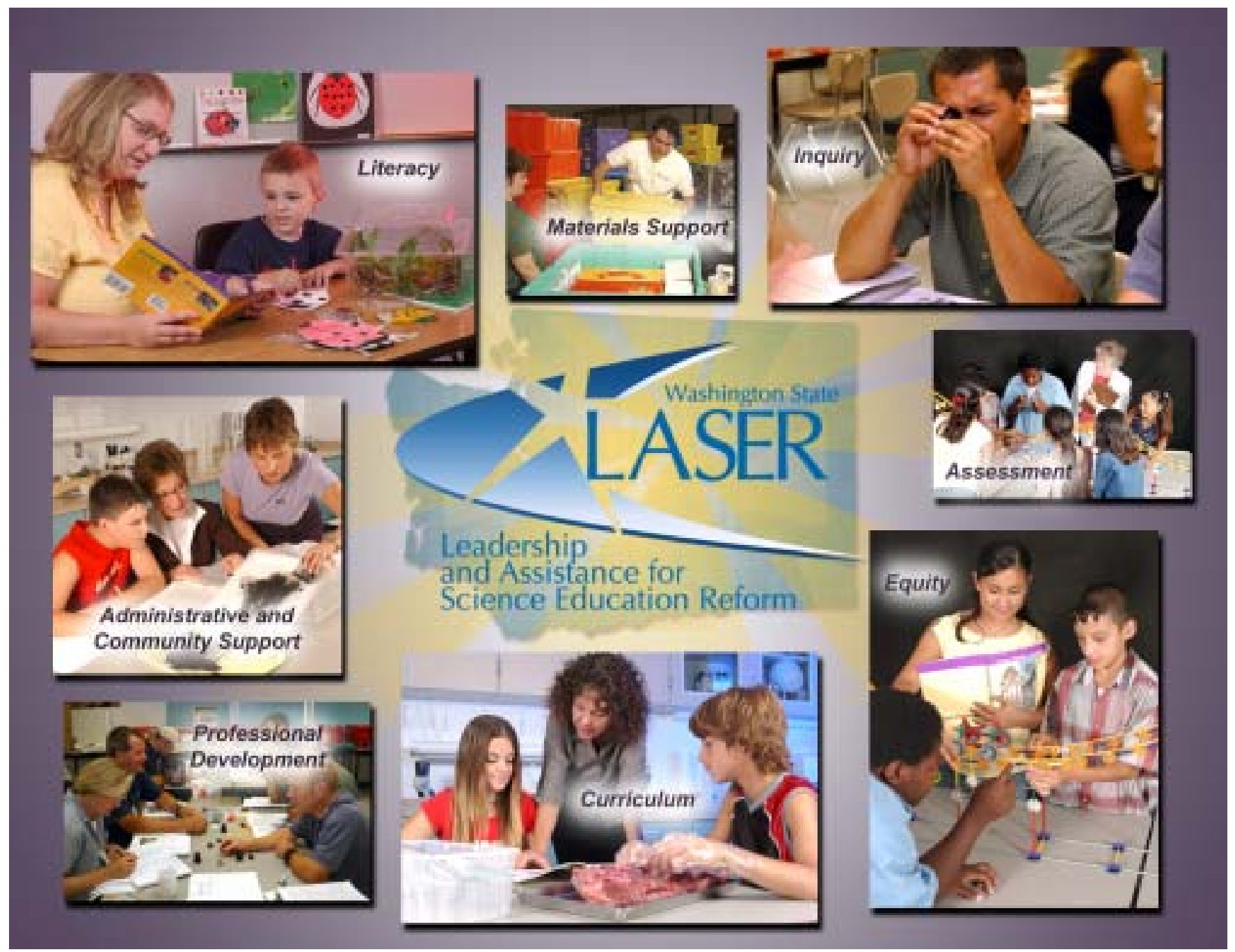

Figure 3.3.1/3.3.2. LASER, based on a successful national model for school improvement, helps school districts design, implement, and sustain K-8 science education reform programs. 



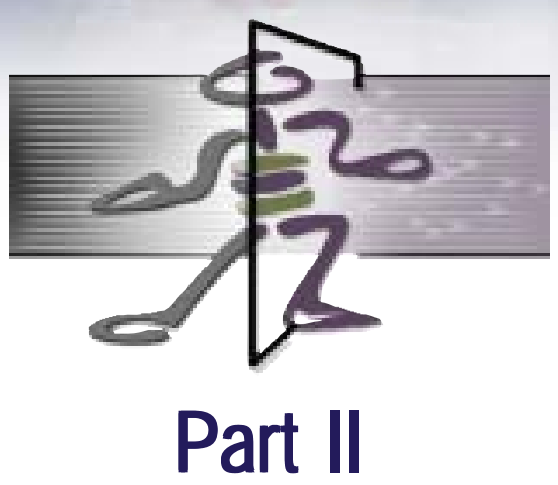

Strengths and Opportunities for Improvement 


\section{II.1 Strengths and Opportunities for Improvement}

We have focused our vision for the Laboratory and are clarifying the strategies for achieving that vision. The vision and emerging strategy build on our core strengths and address opportunities for improvement to ensure that the Laboratory delivers world-class science and technology well into the future. Our new DOE contract and stretch goals reinforce this focus on significant outcomes and provide new mechanisms for managing to those outcomes.

\section{Our Vision and Strategy Builds on Our S\&T Strengths}

Our vision establishes the Laboratory as a leader in integrating physical and chemical sciences with biology to revolutionize the study of living systems and transform that new understanding, and the broader science base of the Lab, into valuable solutions across our programs. The Lab has established S\&T signatures that build on our core capabilities (as described in the 2004-2008 Draft Institutional Plan) and are consistent with the overarching chemical-physical biology focus, around which we will be organizing our investments and from which we will deliver significant new value to our current and future $\mathrm{R} \& \mathrm{D}$ programs. There are currently nine of these signatures, articulating current and emerging strengths of the Laboratory: systems biology and biotechnology, interfacial chemical catalysis, science-driven high performance computing, biogeochemistry and subsurface science, physics-based climate modeling, environmental biomarkers, integrated energy systems, information analytics, and integrated detection, analysis and decision systems.

\section{Additional Organizational and Cultural Strengths that Underlie Our Vision}

As part of our strategy exercise, we interviewed staff and management across the Laboratory, Field Office, Battelle, and among some of our key customers. Those interviews, combined with our own self assessment, highlight the following strengths.

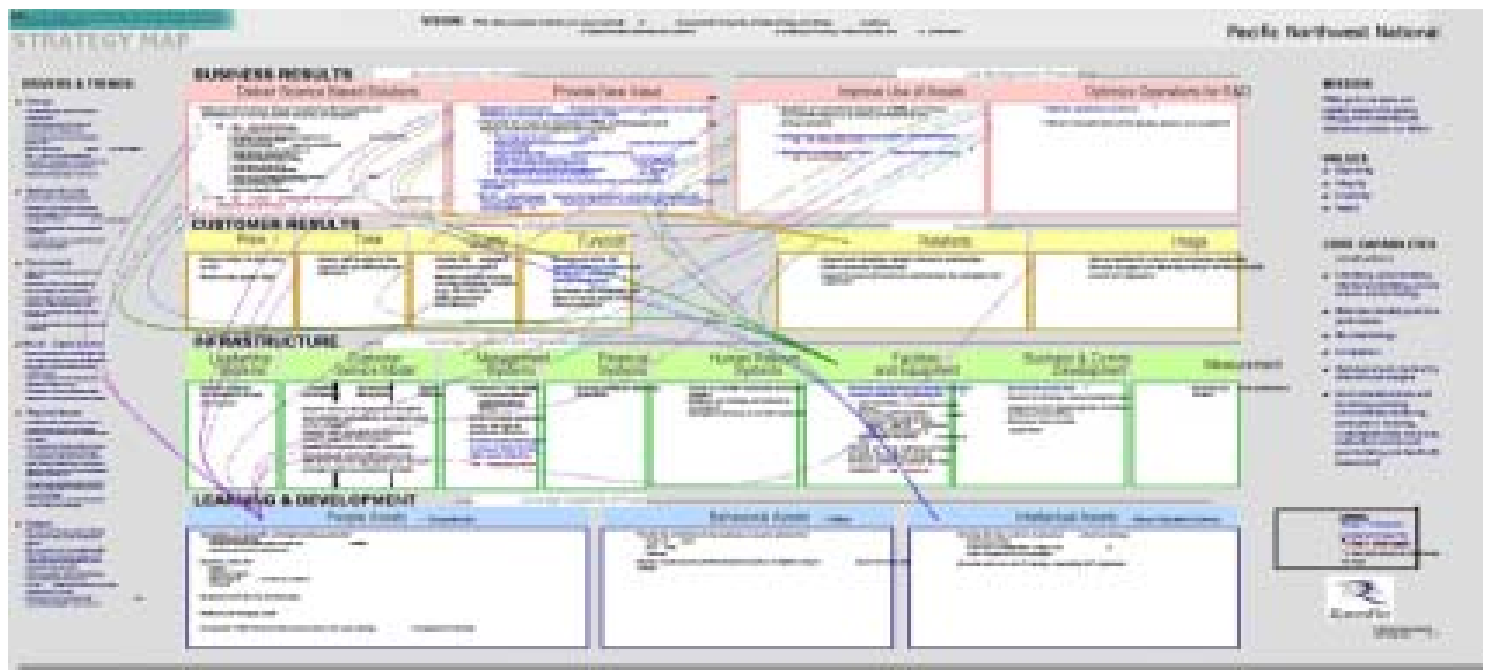

Figure II.1a. Our strategy map communicates the outcomes we want to achieve and the actions we will take to leverage the laboratory systems, processes, and competencies most critical to achieving those outcomes. 
We rapidly translate discoveries into solutions. R\&D 100 and FLC awards attest to our continuing success in translating science into solutions. Three of the five R\&D 100 entries submitted this year received awards. We rank fifth in all federal laboratories in the number of R\&D 100 Awards (62). This year, all three FLC Award entries received awards. We continue to lead federal laboratories in the number of Federal Laboratory Consortium (FLC) Awards (54). Future efforts will focus on developing an IP strategy to support our S\&T signatures through engagement of our technical staff in the development and commercialization of identified solutions.

We generate new $S \& T$ capabilities that anticipate future programs and enable us to deliver highimpact results. Through funded R\&D programs, direct DOE investment, recruitment, and investment of internal PNNL and Battelle overhead funds, we generate new and enhance existing capabilities to poise us to respond to the rapid pace of discovery and innovation that fuels new customer needs and our ability to meet them. Currently, we have eight laboratory-level initiatives to create new or enhance existing capabilities, in bio-molecular systems, nano-science and technology, computational science and engineering, bio-based products, energy systems transformation, carbon management, advanced nuclear science and technology, and homeland security. In addition, we are implementing a lab-level initiative, research campus of the future, to provide the modern research facilities as part of the laboratory's growth agenda. We will also better align our capabilities and investments to support our growth agenda.

We exchange laboratory staff, resources, and ideas to create value across diverse programs. For example, we assembled fundamental science, environmental capabilities, and energy expertise to address critical U.S. national security challenges of detecting and preventing proliferation of weapons of mass destruction. Our knowledge of proliferation signatures provided by the Hanford legacy, experience from long-term missions in environmental monitoring, strong fundamental capabilities in nuclear, radiological, chemical and biological sciences, plus our systems engineering and analysis expertise are being integrated to develop innovative technologies for detecting and preventing the proliferation of weapons of mass destruction - i.e., infrared spectroscopy for application to non-proliferation, chemical sensing field experiments for proliferation detection from ground and air platforms, and mass spectrometry for nonproliferation applications through a fundamentally new multi-element spectrometer than can simultaneously measure All the Signal, All the Time (ASAT).

Summary of Strengths and Opportunities for Improvements
Our thought leadership supports national research agendas. We are leading the development of goals for the GTL program and the GTL facilities functionality roadmap. We are developing answers to important scientific questions through our science grand challenges in biogeochemistry and biology. We are leading the assessment of technical policy options for a wide variety of non-proliferation and arms control programs. Through our leadership of the National Hydrogen Safety Program for DOE's Office of Hydrogen, Fuel Cells, and Infrastructure Technologies, we are helping DOE to define and develop the Hydrogen Safety Program. Our GridWise concepts have served as a compelling vehicle for establishing a set of new activities for the Electricity Distribution Transformation Program in DOE's new Office of Electric Transmission and Distribution.

We acknowledge the critical importance of our staff and managers. We continue to compensate our staff with competitive salaries and a high-quality benefits package. Our Variable Pay Programs (including hiring incentives and individual and team performance awards) enhance our ability to attract and retain qualified staff. In FY2003, we reviewed processes and implemented minor improvements to optimize program performance. We benchmarked our utilization of these programs to ensure that all populations are being rewarded equitably. Our findings indicate that our variable pay programs are on target and are effectively rewarding individual, team, and organizational outcomes that benefit DOE and PNNL. We will continue to monitor program utilization to ensure continued program effectiveness.

Our Rewards and Recognition Program is strong and includes Outstanding Performance Awards (OPAs) and Outstanding Team Performance Awards (OTPAs) in addition to Directorate-level and other reward programs focused on specific laboratory objectives (e.g., intellectual property, commercialization).

We have several programs focused on developing outstanding managers and staff - our Management Skills Development Program, our Workplace Engagement Assessment (Gallup), our Leader/Manager StrengthsFinder Assessment, and the movement of our R\&D/S\&T leaders across Battelle-managed laboratories for the benefit of staff and DOE. Future efforts will focus on strengthening and diversifying our scientific and engineering leadership by creating a work environment that enables S\&T staff to develop exceptional credentials and outcomes, promotes innovation and higher research productivity, and recognizes our technical leaders for their abilities to translate vision into action, and aligns people and science challenge to DOE missions. 


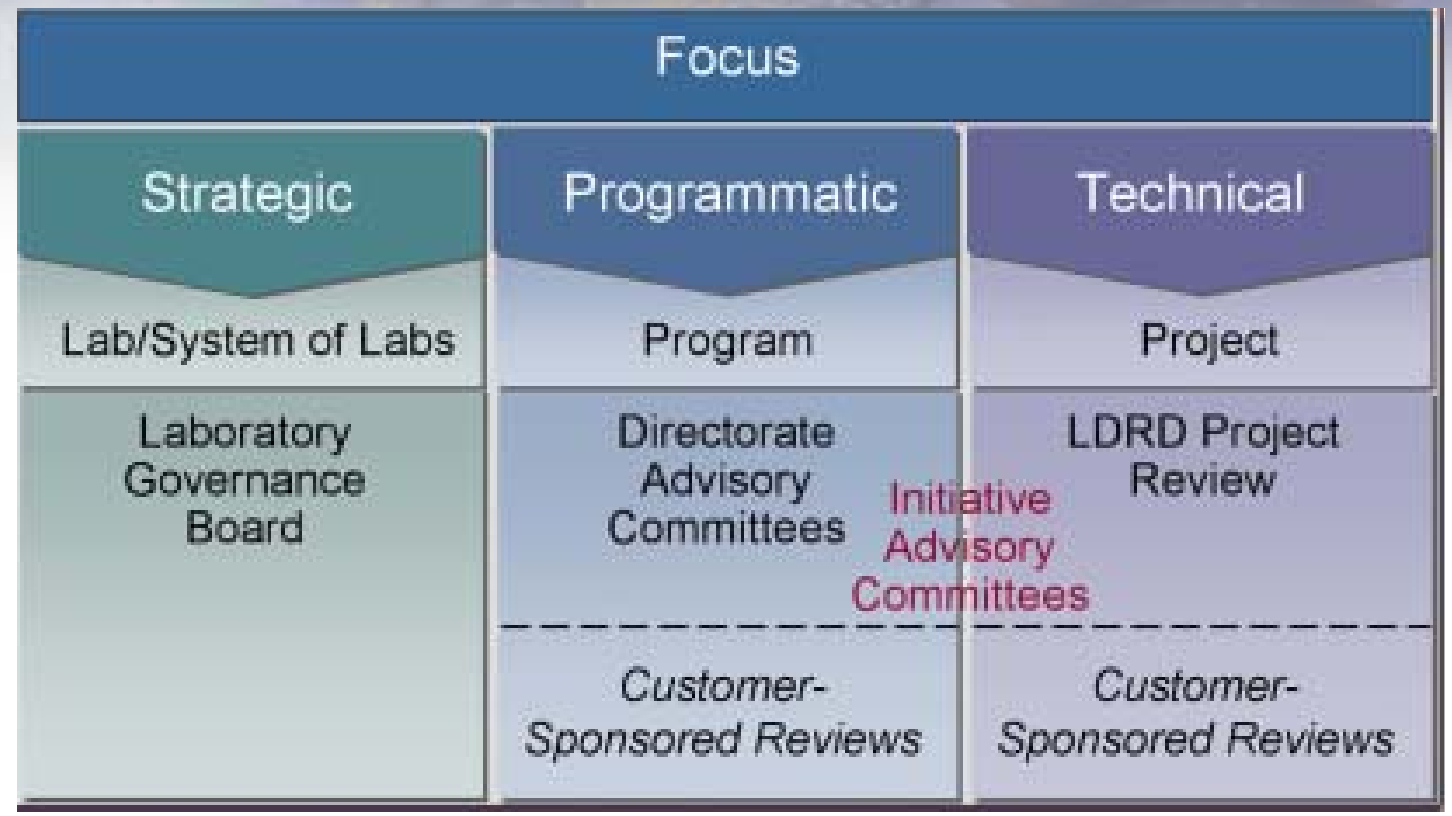

Figure II.1b. Our comprehensive peer review process captures reviews at all levels of the Laboratory.

We have a strong culture of peer review and selfassessment for continual improvement. We have set records within the national laboratory system for external certification and for establishing the basis for contract improvements in laboratory oversight and management. Our comprehensive peer review process includes quarterly reviews of laboratory strategy by a Laboratory Advisory Committee, regularly reviews of the mission/ programmatic areas by Division Review Committees (one committee for each of the four mission areas of Science, National Security, Energy, and Environment), and annual reviews of our projects and initiatives by technical experts, advisors, and customers. Results of our peer reviews are summarized in modules 1.1.1, 1.2.1, 1.3.1, and 1.4.1.

Our self-assessment program is institutionalized. Selfassessments of performance, conducted annually by each laboratory organization, yield results used in the annual business performance review and planning process.

We successfully transfer technologies through unique and flexible IP tools and infrastructure. In FY2003, we generated $\$ 1,326,746$ in revenues derived from DOE inventions-over $47 \%$ more than needed for a rating of outstanding. In addition, other value beyond revenue was generated in "returns" to PNNL (increased R\&D sales and $R \& D$ equipment assets). Our IP toolkit of traditional national laboratory tools (e.g., CRADAs) and tools that other laboratories don't have (e.g., Use Permit), plus a continual focus on enhancing our intellectual property development, management, and licensing activities, increases our ability to respond to industry quickly and creatively.

We bring together the region's major research institutions in focused efforts to find solutions to significant regional issues, build new technology-based enterprises to broaden and diversify the region's economy, and contribute to the region's science and engineering education. Through our leadership of the Linking Regional Resources (a consortium of the region's S\&T institutions) and the use of our Starlight system, we linked S\&T institutions and their resources to find solutions to the region's water resource management needs from among the S\&T resources of its member institutions. Our technology, technical assistance, and entrepreneurial support played a significant part in establishing or expanding six local businesses. Five PNNL initiatives are helping grow and diversify the local and regional technology sector. The Leadership and Assistance for Science Education Reform (LASER) Program enhanced the capacity of school district leadership teams to design and implement their strategic plans for K-8 science education reform. Future efforts will focus on executing a regional strategy that builds broad advocacy through strategic investments and partnerships with universities and industry, corporate contributions, and 
volunteerism, to increase and sustain a modern research campus and an enhanced quality of life in the surrounding community.

\section{Opportunities for Improvement Noted Last Year Have Been Addressed}

We improved our management of the EMSL user facility. The effectiveness and efficiency of our management of EMSL user projects and resources have been significantly improved by implementing two new software applications:

- the EMSL User System (EUS), which facilitates receipt and processing of proposals and communications with users, and

- the EMSL Resource System (ERS), an internal product that tracks instrument use.

In addition, a new EMSL user operations model is already increasing the scientific impact of this unique resource. The model is the basis for a detailed EMSL Facility Operations Manual and emphasizes attracting high-level scientists working in strategically targeted areas.

In anticipation of our new contract, we developed innovative approaches to Laboratory business operations. We streamlined the process and tools associated with proposal pricing, enhanced how the Laboratory obtains low-dollar value purchases through a Vendor Management Program, and implemented an action plan designed to enhance the Laboratory's P-Card.

We continued with enhancements of our $E S H \& Q$ systems and tools to make science and research safer and more efficient. We formally obtained ISO 14001 registration, fully implemented WISHA Ergonomics Rule and developed web-based training for staff, re-engineered our Radioactive Waste Operations program, expanded our keyword index capabilities to enable users of the Standards-Based Management System to find information more quickly, developed and implemented system controls for working with biological materials, and developed web-based training for staff working with select agents.

We better clarified and more broadly communicated the multi-program lab value through our efforts this year to generate active, shared stewardship of our broad resources across DOE and our other key customer sponsors. Shared stewardship involves partner- ships with our major customers to help define and meet mission-driven needs for capability development, research equipment, and research facilities. Through shared stewardship, DOE and our other major customers can collaboratively supply the significantly increased level of support needed to sustain the state-of-the-art research and development capabilities we depend on to deliver on our accomplishments over the next five years. Our efforts to generate shared stewardship included working with DOE-SC and our National and Homeland Security customers to highlight the need and urgency for shared stewardship, and to outline the outcomes and benefits, areas of opportunity, a set of principles, and potential mechanisms. Future efforts will focus on obtaining a commitment from our key customers for shared stewardship of the laboratory's strategy, programmatic oversight, infrastructure investment, and performance evaluation by communicating the outcomes and benefits of such an arrangement and working with them to establish the supporting mechanisms.

To provide the infrastructure and capabilities needed for the future, we worked on improving our ability to anticipate the changing needs of DOE programs and gain consensus of the scientific community, advisory bodies, and/or clients on our responses to those needs. Key enhancements included creating a special Deputy Director for Infrastructure Stewardship within our Facilities and Operations Directorate to engage key customers and stakeholders in our infrastructure planning and to do more comprehensive analysis and business-case development to support change decisions. We also established a Laboratory-level initiative focused on the Research Campus of the Future to provide the necessary attention and resources to ensure that modernization responds to the needs of future DOE and other customer programs.

We better communicated our strategy for (re)investment of IP revenues and resulting DOE benefits to our DOE customer. Through our strategy for reinvestment and monthly meetings with AMT, we discuss our investments on a regular basis. Our strategy is focused on investing our revenues from privately and DOE-funded technology transfer activities to provide resources for S\&T and capability development to meet future DOE and national needs. The revenues fund new capabilities, equipment, and R\&D in DOE and Battelleowned facilities to seed next-generation technologies. Investments are made at all stages of the technology 
development and commercialization (i.e., technology maturation, product development, patenting, and licensing). Our strategy also includes investments we will make with our "use at facility funds" (facilities, equipment, $\mathrm{R} \& \mathrm{D}$, etc.) to benefit the capabilities we can offer to DOE.

We improved our level of recognition in the region as an $S \& T$ advisor. Our work with the LRR consortium has been published in multiple venues, including BIO 2003, The Washington State Senate Technology and Communications Committee Hearing on Technology Transfer, The Northwest Entrepreneurial Network - Biotechnology Meeting, and the Federal Lab Consortium Annual Meeting TechEntreprise 2003.

Our S\&T leadership has also been demonstrated through our activities under our economic development programs. With substantial support from PNNL, six businesses started or expanded offices in the Tri-Cities. PNNL researchers provided no-cost technical assistance to 46 firms in the Northwest region, providing specialized expertise not readily found in the private sector. We implemented five new initiatives to advance the local and regional technology sector. We hosted the Hydrogen Production and Northwest Transportation Conference in Seattle June 2003.

Key partnerships with universities, laboratories, and industry in the region are also increasing our recognition in the region. We have a collaborative program in systems biology with Washington State University-TriCities. We take part in the Collaborative Institute for Bioproducts Research with Washington State University, University of Idaho, the Idaho National Engineering and Environmental Laboratory. Through our Microproducts Breakthrough Institute, we collaborate with Oregon State University. We play a major role in the Northwest Alliance for Transportation Technologies, where we partner with PACCAR and Freightliner.

Our senior scientists have been invited to provide information and participate in briefings to the Washington State legislators on the impacts of regional climate change.

We increased mentoring expectations among our technical staff and improved our educational contributions to K-12 education leaders. Through the LASER Program, we enhanced the capacity of school district leadership teams to design and implement their strategic plans for K-8 science education reform. LASER enabled leadership teams to network with other leadership teams to learn "best practices," provided presentations and workshops that enhanced knowledge and skills, and fostered the development of action plans for science education reform.

We continued to validate our salary data against established market surveys and to look for new sources of benchmark data in the high tech industry. Annually, we obtain external market data through various local, regional, and national salary surveys. The ranges for each level within each job family are reviewed annually by comparing them with the external market data to ensure that our salaries are equitable.

We continued to improve the communication of our lab-level strategic direction and performance expectations. Our strategy mapping has led us to better articulate and communicate our vision and strategic direction. Our vision, outcomes, and strategy have been further communicated through Leadership Council, senior staff, organization staff meetings, and frequent laboratory-wide communications from the Director. Future efforts will focus on enhancing accountability for results by taking the next steps towards establishing a high-performance laboratory-establishing a scorecard or dashboard that communicates accountabilities for and progress on laboratory-level outcomes.

We enhanced tools for project managers to help them deliver products and services (e.g., Hazards Analysis tool). We developed and deployed the new Electronic Prep \& Risk (EPR) system. This new system integrated and enhanced existing hazard analysis tools to better assure the consistent and effective identification, evaluation, and mitigation of hazards; and includes an efficient work authorization process. We rolled out Integrated Operations System (IOPS) concepts and tools to the Marine Science Laboratory in Sequim, completing a multi-year effort to deploy IOPS to all PNNL's labintensive facilities. IOPS communicates safe laboratory practices, identifies and controls workspace hazards, identifies and obtains appropriate training, and authorizes access to workspaces for an efficient and productive laboratory.

We increased our management focus on funds control. We implemented an 8-point action plan that improves documentation, controls, and communication, resulting in a heightened awareness of work authorization requirements by both DOE field and HQ staff, and PNNL staff, and the need to anticipate and quickly resolve work authorization issues.

We improved the quality control of standards for the Analytical Support Operations (ASO) in the Radiochemical Processing Laboratory. In response to QA/ 
QC failures associated with Analytical Support Operations in the Radiochemical Processing Laboratory, we implemented corrective actions and a Laboratory Standards Verifications Plan. The plan was composed of two parts: 1) third-party review of the ASO standards preparation and utilization processes, and 2) $100 \%$ verification of existing standards in use throughout ASO laboratories. All actions and commitments from the Laboratory Standards Verification Plan have been completed.

We helped staff better understand what is expected of them. Through our StrengthsFinder assessments we targeted and are helping our managers understand that staff better understand what is expected of them when managers clearly define and communicate expectations, keep associates informed of decisions that affect their work, frequently interact with their staff, and speak with a consistent voice as a management team. We continue to measure this dimension through our workplace engagement survey each year. Our score indicates we are making steady progress.

We developed a common set of IP performance measures that capture more than just revenues. In addition to measuring our IP commercialization performance in terms of revenues generated, we now also measure the increased R\&D from joint development activities and the value of equipment obtained for the laboratory, either through license agreements or investment of commercialization returns.

\section{Targeted Opportunities for Improvement}

We will increase our science and engineering leadership in the lab by focusing new staff development and training efforts on our S\&E staff, establishing hiring practices aligned with bringing new leaders into the Lab, and further increasing activities to mentor and promote staff within the science community. We will work with our university partners and DOE to further develop our physical-chemical biology signature and stature across the scientific community, focusing on the accomplishment of our DOE stretch goals.
We will enhance our capability and facility planning by developing the mission need as part of our Research Campus of the Future initiative and transition from the 300 Area, obtaining shared stewardship from our major customers for the Laboratory's capabilities, conducting in-depth assessments of strategic capabilities, and better understanding and managing our capacity to grow in important areas.

We will further enhance our regional presence and develop advocacy for the Lab and DOE by expanding our regional university partnerships and developing or strengthening relationships that are critical to influencing $\mathrm{R} \& \mathrm{D}$ strategies and growth in the region.

We will increase our research productivity by driving down our indirect to direct labor ratio through improved operational efficiencies and core R\&D growth; building and retaining a workforce of fully engaged managers and staff with a mindset for continued operational excellence, the ability to substantially contribute to DOE and Laboratory outcomes, and a clear focus on scientific productivity; and broadly communicating our vision and strategy within the Lab.

We will upgrade Integrated Assessment to support new laboratory-level assurance requirements. Key actions we will take include establishing and communicating a new governance approach, implementing our dashboard, implementing our risk management approach, and obtaining additional external certification.

We will pursue our continued improvement initiative to work together with DOE to develop and implement innovative approaches and techniques for improving contract performance and administration. We will evaluate elimination/reduction of mandatory Hanford Site Services, contractor provided facilities, the consolidated lab concept and administration, our policies and procedures related to technology transfer, enhancements to our variable pay incentive programs, and reciprocity of benefits and pension. 



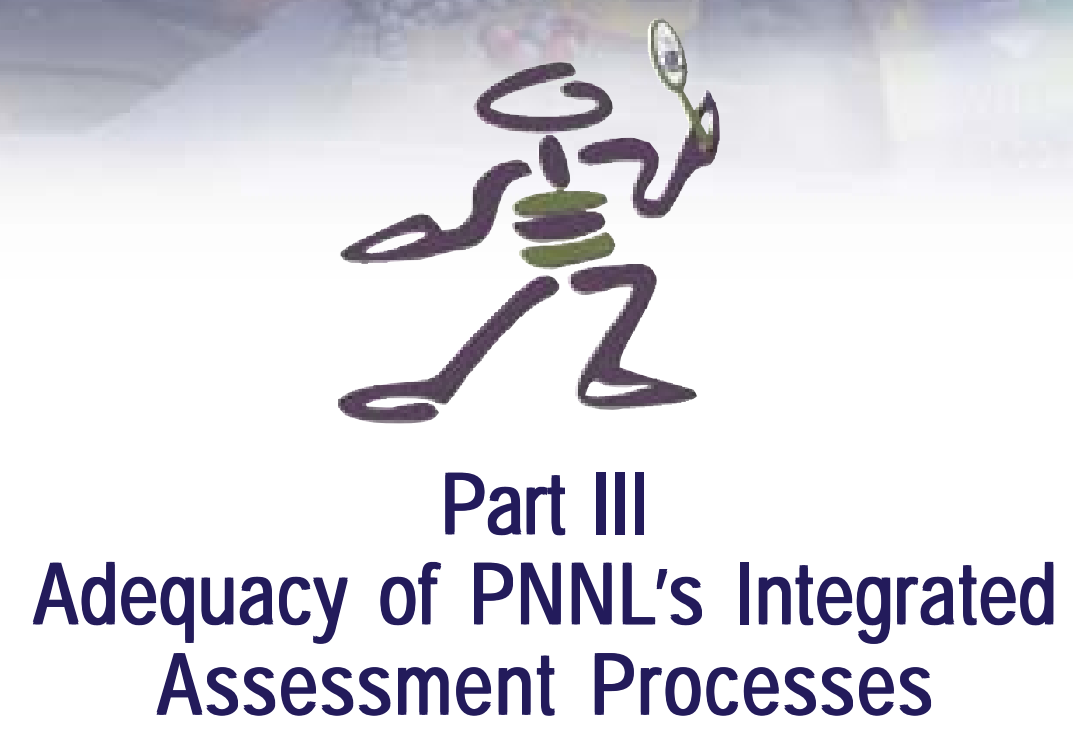




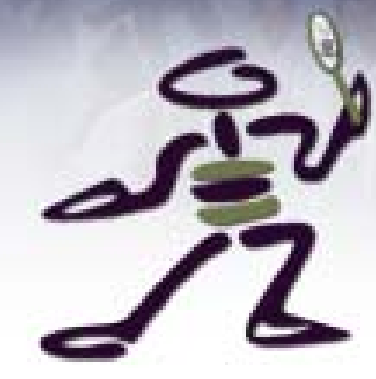

III.1 Adequacy of Integrated Assessment
Processes to Establish Compliance
with Key Internal Controls

PNNL's robust process for promulgating appropriate contractual and regulatory requirements and assessing performance against them forms a strong foundation to meet DOE expectations for corporate assurance. This process will be further improved during FY2004 by systematizing internal controls for assurance at the Battelle Corporate level.

\section{How PNNL Promulgates Requirements and Assesses Compliance}

Our requirements management process is effective in promulgating appropriate external requirements (DOE Directives and Federal, state, and local laws) through our Standards Based Management System, which guides work in the Laboratory. Management System Owners (MSOs) determine applicability of new or revised external requirements, how the Laboratory will comply, and the actions needed to achieve compliance. These may include developing or revising subject areas, program descriptions, task- and group-specific procedures, or other actions. The actions taken to implement requirements are recorded on a Record of Decision (ROD) and tracked.

Our integrated assessment processes evaluate implementing methods to assure that requirements are being met. We rely on self-assessment, independent oversight, and internal audit to provide us assurance that business objectives are being met and risks associated with the work are being managed. External reviews by customers, stakeholders, and regulators provide additional indicators of the robustness of our processes to find and manage needed improvements. Table III.1 shows the number of findings external reviewers found in FY2003 that we had not previously found through our selfassessment. In FY2002, there were no significant findings by external audits that we had not previously found. This year, external review findings identified 11 previously unidentified areas for improvement that were in the area of unclassified cyber security. A cyber security task team was established to formally expedite corrective actions. We continue to be confident in the rigor of our approach to integrated assessment and our ability to rapidly respond to needed improvements.

\section{Focusing on What's Most Important}

With 354 requirements to promulgate and manage throughout the Laboratory, we must review performance to those requirements on a routine but selective basis to manage costs. (For compliance with requirements not selected for formal review, we rely on self-assessment to alert us to emerging issues and needed improvements at our local working groups, project, program, and directorate levels. Self-assessments are done on an on-going basis throughout the entire Laboratory to provide information on these issues or improvements.) Independent Oversight and Internal Audit Managers review a subset of our core processes and key internal controls each year for senior leadership in the Laboratory and our corporate office. The Deputy Laboratory Director of Operations assures direct and immediate communication of significant issues and non-compliances with the entire Leadership Council by regularly meeting with Directors of the operations directorates (e.g. ESH\&Q, HR, Facilities) and R\&D Operations Managers, who provide self-assessment derived issues or improvement needs. The Deputy Laboratory Director of Operations also meets with the Managers of Internal Audit and Independent Oversight, who communicate issues or improvement needs derived from key process and internal control reviews. In this way, all senior executives keep abreast of emerging issues and needed improvements and can direct resources to assure performance is changed as needed. (Figure III.1)

\section{Self-Assessment and the 10 Special Study}

The study, Analysis of Laboratory Incidents June 2003, identified the need to improve our awareness and identification of hazards and risks. In response, our ALDs and ESH\&Q and F\&O Directors are committed to increase their presence and that of their Technical Group 
Managers in the workplace. They will use questions directly to staff during their walkthroughs to raise awareness. Independent Oversight will do a follow-up study in FY2004 to assure these actions are carried out and ascertain their effectiveness.

\section{PNNL's Operations: Effective and Compliant}

The IO Special Study of Integrated Operations System (IOPS), March 2003, highlights the high value and effective implementation of our IOPS program. This program helps us stay compliant with many operational requirements. Our staff highly values the system for its ability to help them identify and manage operational risk and is complying with the requirements of the system.

Internal Audit identified an opportunity for improvement in our accountability and oversight of costs being incurred for cell phones, broadband use, and p-cards. As a result, we are strengthening our management oversight and requiring stronger business justification for costs in these areas.

We have excelled at operations and compliance over the past five years because of our commitment to continual improvement. We seek out areas of weakness or noncompliance and address them directly to maintain a high-performance Laboratory for the U.S. Department of Energy. With the signing of our new contract in FY2003, we will continue systematizing our internal controls by enhancing governance and assurance processes for the Laboratory. We have always participated in corporate-lead reviews of specific operational areas, but in FY2004 this will be significantly expanded to other areas of interest by our corporate office. In FY2003, the Corporate Laboratory Operations Council was formed to address laboratory operations of all Battelle-affiliated labs. This was the first step in a longerterm process to address a new contractual requirement for PNNL - a corporate letter that provides reasonable assurance that our management systems are effective and efficient. An assurance process will be developed for review and approval by the DOE contracting officer for implementation in FY2004.

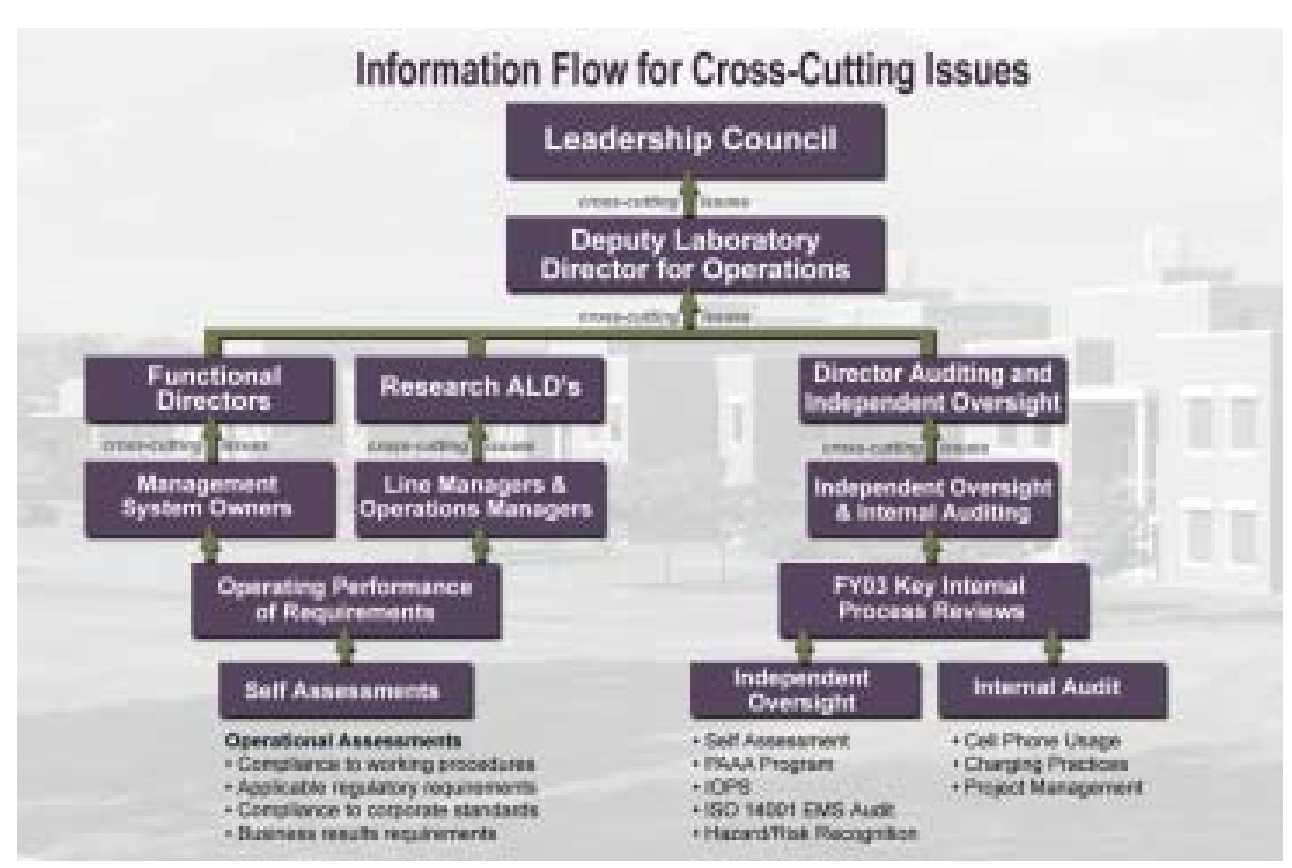

Figure III.1. The Deputy Laboratory Director of Operations assures direct and immediate communication of significant issues and non-compliances with senior management by regularly meeting with Directors of the operations directorates (e.g. ESH\&Q, HR, Facilities), R\&D Operations Managers, and Managers of Internal Audit and Independent Oversight.

Table III.1. FY03 external reviews provide confidence that very few findings are not previously identified by PNNL self-assessments.

\begin{tabular}{|c|c|c|c|c|c|c|}
\hline & $\begin{array}{l}\text { Number } \\
\text { Reviews }\end{array}$ & $\begin{array}{l}\text { Number } \\
\text { Assessors }\end{array}$ & $\begin{array}{c}\text { Number } \\
\text { PNNL } \\
\text { Person-days }\end{array}$ & $\begin{array}{l}\text { Number } \\
\text { Findings }\end{array}$ & $\begin{array}{l}\text { Number } \\
\text { Significant } \\
\text { Findings } \\
\text { Not Found } \\
\text { by PNNL }\end{array}$ & $\begin{array}{l}\text { Number of } \\
\text { Findings } \\
\text { With No } \\
\text { Response } \\
\text { Required }\end{array}$ \\
\hline $\begin{array}{l}\text { FY03 External Reviews(a) } \\
\text { Facility Rep Reviews }\end{array}$ & $\begin{array}{l}27 \\
30\end{array}$ & $\begin{array}{c}101 \\
2\end{array}$ & $\begin{array}{l}1149(5 \text { FTEs }) \\
\text { Not tracked }\end{array}$ & $\begin{array}{l}30 \\
44\end{array}$ & $\begin{array}{c}11 \\
\text { Not tracked }\end{array}$ & $\begin{array}{l}\mathrm{N} / \mathrm{A} \\
26\end{array}$ \\
\hline
\end{tabular}

(a) Includes: WA State Dept. of Ecology, WA State Dept. of Health, City of Richland, DCAA, Battelle Corporate, US DOE Office of Oversight and Performance Assurance, US DOE HQ ICCP, DEA, WA State Board of Pharmacy. 



\section{Appendix A \\ Critical Outcome Performance Ratings}




\section{Appendix A - Critical Outcome Performance Ratings}

Battelle's performance rating for FY2003 is developed by determining the year-end level of performance for each performance indicator, compared to the individual targets established in the FY2003 Performance Evaluation \& Fee Agreement. This level of performance is then judged against the metrics developed for each performance indicator and an appropriate adjectival rating is assigned. The adjectival rating for each performance indicator is inserted into the rating tables and Value Points are assigned, based on the following scale:

$\begin{array}{ll}\text { Outstanding } & 4 \text { points } \\ \text { Excellent } & 3 \text { points } \\ \text { Good } & 2 \text { points } \\ \text { Marginal } & 1 \text { point } \\ \text { Unsatisfactory } & 0 \text { points }\end{array}$

The Value Points are added to the rating tables and are multiplied by the weight of each performance indicator and then added to develop the Objective score. The Objective scores are then multiplied by the Objective weightings and are added to develop the overall score for the Critical Outcome. The values from the individual Critical Outcome tables found in this Appendix are then transferred to Table A.1, and compared against the ranges found in Table A.2 to determine the Critical Outcome (adjectival) performance rating.

The individual (weighted) Critical Outcome scores are summed to determine the overall Laboratory score. This value is compared against the ranges found in Table A.2 to determine the Laboratory's overall FY2003 (adjectival) performance rating.

All numbers are rounded to the nearest hundredth and carried forward to the final Laboratory overall all rating, which is then rounded to the nearest tenth, using standard rounding convention.

Table A.1. FY2003 Overall Performance Score

\begin{tabular}{|l|c|c|c|c|}
\hline Critical Outcome & $\begin{array}{c}\text { Value } \\
\text { Points }\end{array}$ & Adjectival Rating & Weight & $\begin{array}{c}\text { Weighted } \\
\text { Score }\end{array}$ \\
\hline $\begin{array}{l}\text { Scientific and Technological } \\
\text { Excellence }\end{array}$ & 3.98 & Outstanding & $60 \%$ & 2.39 \\
Management and Operations & 3.88 & Outstanding & $25 \%$ & 0.97 \\
Excellence & 3.70 & Outstanding & $15 \%$ & 0.56 \\
Leadership Excellence & & Total Score & 3.9 \\
\hline
\end{tabular}

Table A.2. FY2003 Adjectival Rating Scale

\begin{tabular}{|l|c|c|c|c|c|}
\hline Total Score & $4.0-3.5$ & $3.4-2.5$ & $2.4-1.5$ & $1.4-0.5$ & $<0.5$ \\
\hline Final Rating & Outstanding & Excellence & Good & Marginal & Unsatisfactory \\
\hline
\end{tabular}


Table A.3. Science and Technological Excellence Critical Outcome Performance Rating

\begin{tabular}{|c|c|c|c|c|c|c|}
\hline Element & $\begin{array}{l}\text { Adjectival } \\
\text { Rating }\end{array}$ & $\begin{array}{l}\text { Value } \\
\text { Points }\end{array}$ & $\begin{array}{l}\text { Indicator } \\
\text { Weight }\end{array}$ & $\begin{array}{l}\text { Total } \\
\text { Points }\end{array}$ & $\begin{array}{l}\text { Objective } \\
\text { Weight }\end{array}$ & $\begin{array}{l}\text { Total } \\
\text { Points }\end{array}$ \\
\hline \multicolumn{7}{|l|}{ 1.0 Scientific and Technological Excellence } \\
\hline $\begin{array}{l}\text { Objectives } 1.1 \text { through 1.4: Program } \\
\text { Office Total Scores (from Table A.4) }\end{array}$ & Outstanding & & & 4.00 & $80 \%$ & 3.20 \\
\hline \multicolumn{7}{|l|}{$\begin{array}{l}1.5 \text { Create leading-edge scientific capabilities to } \\
\text { support evolving DOE Mission needs }\end{array}$} \\
\hline $\begin{array}{l}\text { 1.5.1 Progress against Biomolecular Systems } \\
\text { Initiative expected outcomes (roll up from Table A.5) }\end{array}$ & Outstanding & 4.00 & $50 \%$ & 2.00 & & \\
\hline $\begin{array}{l}\text { 1.5.2 Progress against Computational Sciences } \\
\text { and Engineering Initiative expected outcomes } \\
\text { (roll up from Table A.6) }\end{array}$ & Outstanding & 4.00 & $35 \%$ & 1.40 & & \\
\hline \multirow[t]{2}{*}{$\begin{array}{l}\text { 1.5.3 Progress against the Nanoscience and } \\
\text { Nanotechnology Initiative expected outcomes } \\
\text { (roll up from Table A.7) }\end{array}$} & Outstanding & 3.55 & $15 \%$ & 0.53 & & \\
\hline & & \multicolumn{2}{|c|}{ Objective 1.5 Total } & 3.93 & $10 \%$ & 0.39 \\
\hline \multicolumn{7}{|l|}{1.6 Improve scientific impact of EMSL user program } \\
\hline $\begin{array}{l}1.6 .1 \text { Increase the impact of the EMSL User Program } \\
\text { by establishing science grand challenges that engage } \\
\text { high visibility user communities. }\end{array}$ & Outstanding & 3.70 & $50 \%$ & 1.85 & & \\
\hline \multirow[t]{3}{*}{$\begin{array}{l}\text { 1.6.2 Develop and implement an optimal model for } \\
\text { EMSL user facility operations (user program). }\end{array}$} & Outstanding & 4 & $50 \%$ & 2.00 & & \\
\hline & & Objectiv & e 1.6 Total & 3.85 & $10 \%$ & 0.39 \\
\hline & & \multicolumn{4}{|c|}{ Critical Outcome 1.0 Total } & 3.98 \\
\hline
\end{tabular}

Table A.4. Objectives 1.1 through 1.4 Evaluation Score Calculation for Program Offices

\begin{tabular}{|c|c|c|c|c|}
\hline HQ Program Office & $\begin{array}{l}\text { Value } \\
\text { Points }\end{array}$ & Adjectival Rating & Weight & $\begin{array}{l}\text { Weighted } \\
\text { Score }\end{array}$ \\
\hline Office of Science & 4 & Outstanding & $30 \%$ & 1.20 \\
\hline $\begin{array}{l}\text { Assistant Secretary for } \\
\text { Environmental Management }\end{array}$ & 4 & Outstanding & $20 \%$ & 0.80 \\
\hline $\begin{array}{l}\text { Office of Defense Nuclear } \\
\text { Nonproliferation }\end{array}$ & 4 & Outstanding & $20 \%$ & 0.80 \\
\hline Office of Intelligence & 4 & Outstanding & $5 \%$ & 0.20 \\
\hline Office of Counterintelligence & 4 & Outstanding & $5 \%$ & 0.20 \\
\hline $\begin{array}{l}\text { Assistant Secretary for Energy } \\
\text { Efficiency and Renewable } \\
\text { Energy }\end{array}$ & 4 & Outstanding & $10 \%$ & 0.40 \\
\hline $\begin{array}{l}\text { Assistant Secretary for Fossil } \\
\text { Energy }\end{array}$ & 4 & Outstanding & $10 \%$ & 0.40 \\
\hline & & \multicolumn{2}{|c|}{ Overall Program Office Total } & 4.00 \\
\hline
\end{tabular}


Table A.5. Performance Indicator 1.5.1 Score Calculation

\begin{tabular}{|c|c|c|c|c|c|}
\hline Element & $\begin{array}{l}\text { Value } \\
\text { Points }\end{array}$ & Adjectival Rating & Weight & $\begin{array}{l}\text { Weighted } \\
\text { Score }\end{array}$ & $\begin{array}{l}\text { Overall } \\
\text { Weighted } \\
\text { Score }\end{array}$ \\
\hline \multicolumn{6}{|l|}{$\begin{array}{l}\text { 1.5.1 Progress against } \\
\text { Biomolecular Systems } \\
\text { Initiative expected outcomes }\end{array}$} \\
\hline 1.5.1.1 Recruiting & 4 & Outstanding & $20 \%$ & 0.80 & \\
\hline $\begin{array}{l}\text { 1.5.1.2 Program development } \\
\text { and scientific partnerships }\end{array}$ & 4 & Outstanding & $20 \%$ & 0.80 & \\
\hline $\begin{array}{l}\text { 1.5.1.3 Technical } \\
\text { achievements }\end{array}$ & 4 & Outstanding & $20 \%$ & 0.80 & \\
\hline $\begin{array}{l}\text { 1.5.1.4 Continues technical } \\
\text { and scientific progress }\end{array}$ & 4 & Outstanding & $20 \%$ & 0.80 & \\
\hline 1.5.1.5 Peer review & 4 & Outstanding & $20 \%$ & 0.80 & \\
\hline
\end{tabular}

Table A.6. Performance Indicator 1.5.2 Score Calculation

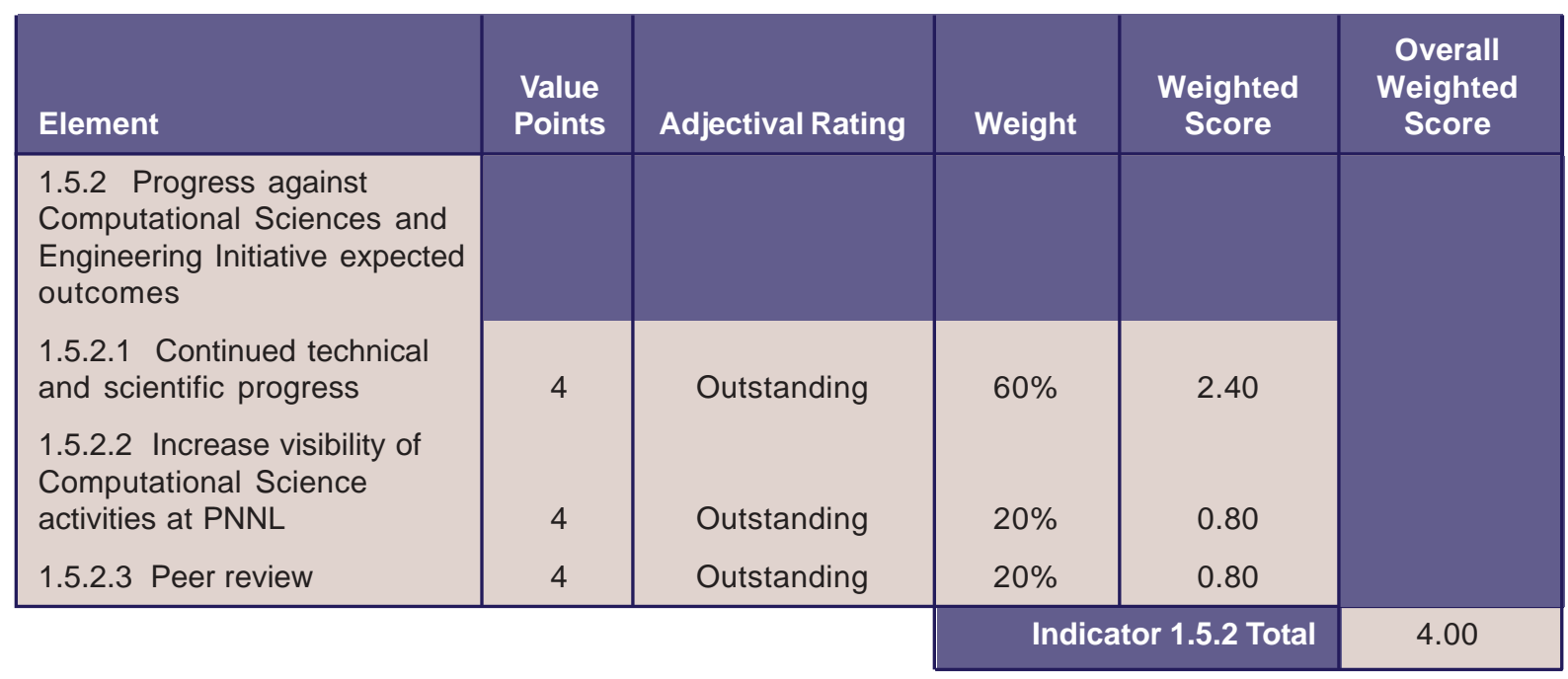

Table A.7. Performance Indicator 1.5.3 Score Calculation

\begin{tabular}{|c|c|c|c|c|c|}
\hline Element & $\begin{array}{l}\text { Value } \\
\text { Points }\end{array}$ & Adjectival Rating & Weight & $\begin{array}{l}\text { Weighted } \\
\text { Score }\end{array}$ & $\begin{array}{c}\text { Overall } \\
\text { Weighted } \\
\text { Score }\end{array}$ \\
\hline \multicolumn{6}{|l|}{$\begin{array}{l}\text { 1.5.3 Progress against the } \\
\text { Nanoscience and } \\
\text { Nanotechnology expected } \\
\text { outcomes }\end{array}$} \\
\hline $\begin{array}{l}\text { 1.5.3.1 Increase visibility of } \\
\text { Nanoscience and } \\
\text { Nanotechnology activities at } \\
\text { PNNL }\end{array}$ & 4 & Outstanding & $33 \%$ & 1.32 & \\
\hline $\begin{array}{l}\text { 1.5.3.2 Project and program } \\
\text { development }\end{array}$ & 3.67 & Outstanding & $33 \%$ & 1.21 & \\
\hline 1.5.3.3 Scientific impact & 3 & Excellent & $34 \%$ & 1.02 & \\
\hline \multicolumn{5}{|c|}{ Indicator 1.5.3 Total } & 3.55 \\
\hline
\end{tabular}


Table A.8. Management and Operational Excellence Critical Outcome Performance Rating

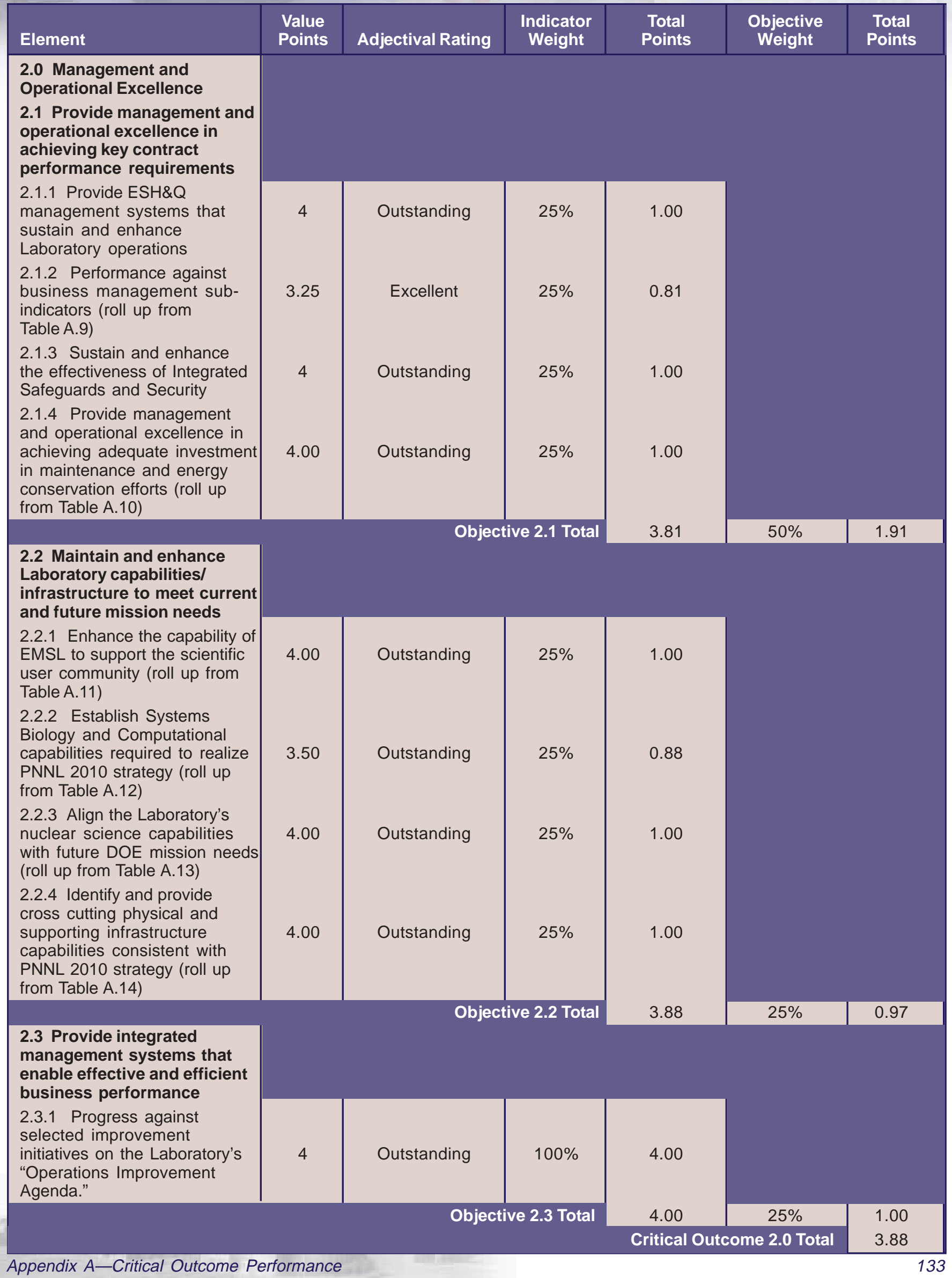


Table A.9. Performance Indicator 2.1.2 Score Calculation

\begin{tabular}{|l|c|c|c|c|c|}
\hline Element & $\begin{array}{c}\text { Value } \\
\text { Points }\end{array}$ & Adjectival Rating & Weight & $\begin{array}{c}\text { Weighted } \\
\text { Score }\end{array}$ & $\begin{array}{c}\text { Overall } \\
\text { Weighted } \\
\text { Score }\end{array}$ \\
\hline $\begin{array}{l}2.1 .2 \text { Performance against } \\
\text { Business Management } \\
\text { sub-indicators }\end{array}$ & \multicolumn{3}{|l|}{} & \\
$\begin{array}{l}\text { 2.1.2.1 Cost Management } \\
\text { Trends: Overhead cost as a } \\
\text { percent of Laboratory's 1830 } \\
\text { fully-burdened average } \\
\text { charge-out rate }\end{array}$ & 4 & Outstanding & $50 \%$ & 2.00 & \\
$\begin{array}{l}2.1 .2 .2 \text { Cost Management } \\
\text { Trends: Labor Overhead as a } \\
\text { multiplier on the 1830 direct } \\
\text { charged operating labor costs } \\
\text { 2.1.2.3 Resource } \\
\text { Management Trends: Direct }\end{array}$ & 2 & Good & $25 \%$ & 0.50 & \\
$\begin{array}{l}\text { FTEs as a percent of the total } \\
\text { Laboratory FTEs }\end{array}$ & 3 & Excellent & $25 \%$ & 0.75 & \\
\hline
\end{tabular}

Table A.10. Performance Indicator 2.1.4 Score Calculation

\begin{tabular}{|c|c|c|c|c|c|}
\hline Element & $\begin{array}{l}\text { Value } \\
\text { Points }\end{array}$ & Adjectival Rating & Weight & $\begin{array}{l}\text { Weighted } \\
\text { Score }\end{array}$ & $\begin{array}{c}\text { Overall } \\
\text { Weighted } \\
\text { Score }\end{array}$ \\
\hline \multicolumn{6}{|l|}{$\begin{array}{l}\text { 2.1.4 Provide management } \\
\text { and operational excellence in } \\
\text { achieving investment in } \\
\text { maintenance and energy } \\
\text { conservation efforts }\end{array}$} \\
\hline 2.1.4.1 Stewardship Index & 4 & Outstanding & $60 \%$ & 2.40 & \\
\hline $\begin{array}{l}\text { 2.1.4.2 Identification and } \\
\text { implementation of energy } \\
\text { conservation measures that } \\
\text { are commensurate with the } \\
\text { Laboratory's strategy to } \\
\text { establish a sustainable } \\
\text { environment for conducting } \\
\text { research and development }\end{array}$ & 4 & Outstanding & $40 \%$ & 1.60 & \\
\hline \multicolumn{5}{|c|}{ Indicator 2.1.4 Total } & 4.00 \\
\hline
\end{tabular}


Table A.11. Performance Indicator 2.2.1 Score Calculation

\begin{tabular}{|c|c|c|c|c|c|}
\hline Element & $\begin{array}{l}\text { Value } \\
\text { Points }\end{array}$ & Adjectival Rating & Weight & $\begin{array}{l}\text { Weighted } \\
\text { Score }\end{array}$ & $\begin{array}{l}\text { Overall } \\
\text { Weighted } \\
\text { Score }\end{array}$ \\
\hline \multicolumn{6}{|l|}{$\begin{array}{l}\text { 2.2.1 Enhance the capability } \\
\text { of EMSL to support the } \\
\text { scientific user community }\end{array}$} \\
\hline $\begin{array}{l}\text { 2.2.1.1 Develop facility and } \\
\text { capability activities for effective } \\
\text { operation of the } 900 \mathrm{MHz} \\
\text { magnet. }\end{array}$ & 4 & Outstanding & $50 \%$ & 2.00 & \\
\hline $\begin{array}{l}\text { 2.2.1.2 Enhance the } \\
\text { Environmental Molecular } \\
\text { Sciences Laboratories ability } \\
\text { to meet its users and DOE's } \\
\text { computational challenges by } \\
\text { installing the HP } \\
\text { supercomputer. }\end{array}$ & 4 & Outstanding & $50 \%$ & 2.00 & \\
\hline \multicolumn{5}{|c|}{ Indicator 2.2.1 Total } & 4.00 \\
\hline
\end{tabular}

Table A.12. Performance Indicator 2.2.2 Score Calculation

\begin{tabular}{|c|c|c|c|c|c|}
\hline Element & $\begin{array}{l}\text { Value } \\
\text { Points }\end{array}$ & Adjectival Rating & Weight & $\begin{array}{c}\text { Weighted } \\
\text { Score }\end{array}$ & $\begin{array}{l}\text { Overall } \\
\text { Weighted } \\
\text { Score }\end{array}$ \\
\hline \multicolumn{6}{|l|}{$\begin{array}{l}2.2 .2 \text { Establish Systems } \\
\text { Biology and Computational } \\
\text { capabilities required to realize } \\
\text { PNNL } 2010 \text { strategy }\end{array}$} \\
\hline $\begin{array}{l}\text { 2.2.2.1 Establish an operating } \\
\text { pilot proteomics facility in } \\
\text { LSL-II }\end{array}$ & 4 & Outstanding & $50 \%$ & 2.00 & \\
\hline $\begin{array}{l}\text { 2.2.2.2 Provide adequate } \\
\text { capability to meet the }\end{array}$ & & . & & & \\
\hline $\begin{array}{l}\text { Computational Science needs } \\
\text { across major PNNL research } \\
\text { areas }\end{array}$ & 3 & Excellent & $50 \%$ & 1.50 & \\
\hline & & & Indic & 2.2.2 Total & 3.50 \\
\hline
\end{tabular}


Table A.13. Performance Indicator 2.2.3 Score Calculation

\begin{tabular}{|c|c|c|c|c|c|}
\hline Element & $\begin{array}{l}\text { Value } \\
\text { Points }\end{array}$ & Adjectival Rating & Weight & $\begin{array}{l}\text { Weighted } \\
\text { Score }\end{array}$ & $\begin{array}{l}\text { Overall } \\
\text { Weighted } \\
\text { Score }\end{array}$ \\
\hline \multicolumn{6}{|l|}{$\begin{array}{l}\text { 2.2.3 Align the Laboratory's } \\
\text { nuclear science capabilities } \\
\text { with future DOE mission needs }\end{array}$} \\
\hline $\begin{array}{l}\text { 2.2.3.1 Identify and implement } \\
\text { measures that are commen- } \\
\text { surate with the Laboratory's } \\
\text { strategy to consolidate and } \\
\text { sustain radiological and radio- } \\
\text { chemical capabilities }\end{array}$ & 4 & Outstanding & $50 \%$ & 2.00 & \\
\hline $\begin{array}{l}\text { 2.2.3.2 Establish new, joint } \\
\text { research and development } \\
\text { projects with academia, using }\end{array}$ & & & & & \\
\hline $\begin{array}{l}\text { PNNL's nuclear capabilities, } \\
\text { which will provide opportunities } \\
\text { to develop and recruit future } \\
\text { nuclear scientists and } \\
\text { engineers. }\end{array}$ & 4 & Outstanding & $50 \%$ & 2.00 & \\
\hline \multicolumn{5}{|c|}{ Indicator 2.2.3 Total } & 4.00 \\
\hline
\end{tabular}

Table A.14. Performance Indicator 2.2.4 Score Calculation

\begin{tabular}{|c|c|c|c|c|c|}
\hline Element & $\begin{array}{l}\text { Value } \\
\text { Points }\end{array}$ & Adjectival Rating & Weight & $\begin{array}{l}\text { Weighted } \\
\text { Score }\end{array}$ & $\begin{array}{l}\text { Overall } \\
\text { Weighted } \\
\text { Score }\end{array}$ \\
\hline \multicolumn{6}{|l|}{$\begin{array}{l}\text { 2.2.4 Identify and provide } \\
\text { cross cutting physical and } \\
\text { supporting infrastructure } \\
\text { capabilities consistent with } \\
\text { PNNL } 2010 \text { strategy }\end{array}$} \\
\hline $\begin{array}{l}\text { 2.2.4.1 Increase internet } \\
\text { connection to accommodate } \\
\text { strategic research collabora- } \\
\text { tions requiring extensive } \\
\text { computation and transfer of } \\
\text { large data sets }\end{array}$ & 4 & Outstanding & $20 \%$ & 0.80 & \\
\hline $\begin{array}{l}\text { 2.2.4.2 Increase computer } \\
\text { network capability to accom- } \\
\text { modate strategic classified } \\
\text { research collaborations } \\
\text { requiring extensive computa- } \\
\text { tion and transfer of large data } \\
\text { sets }\end{array}$ & 4 & Outstanding & $20 \%$ & 0.80 & \\
\hline $\begin{array}{l}\text { 2.2.4.3 Develop and } \\
\text { commence implementation } \\
\text { of a strategy to maintain } \\
\text { continuity of the Department } \\
\text { of Energy science mission } \\
\text { while enabling accelerated } \\
\text { cleanup of the Hanford } \\
300 \text { Area. }\end{array}$ & 4 & Outstanding & $60 \%$ & 2.40 & \\
\hline \multicolumn{5}{|c|}{ Indicator 2.2.4 Total } & 4.00 \\
\hline
\end{tabular}


Table A.15. Leadership Excellence Critical Outcome Performance Ratings

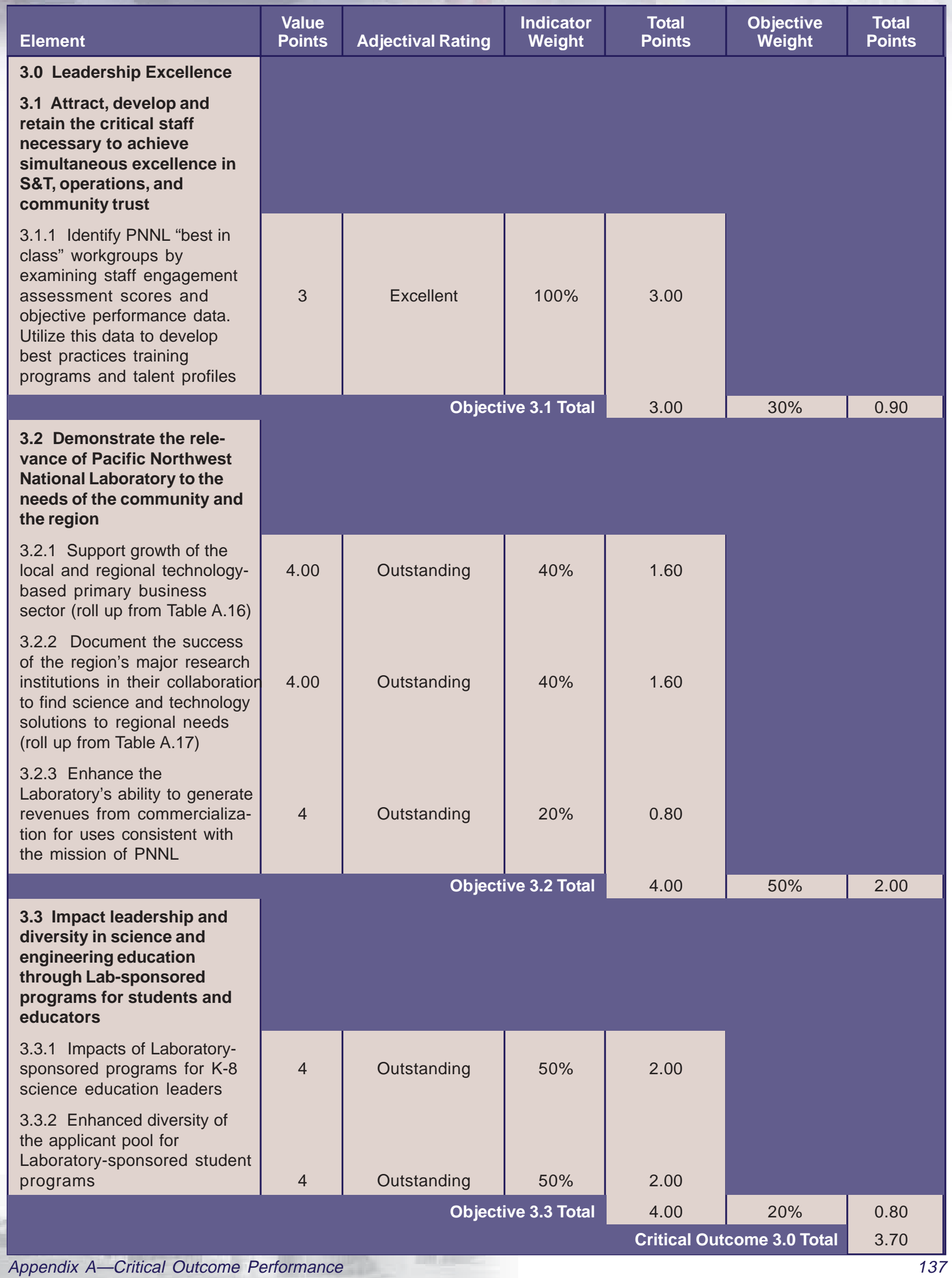


Table A.16. Performance Indicator 3.2.1 Score Calculation

\begin{tabular}{|l|c|c|c|c|c|}
\hline Element & $\begin{array}{c}\text { Value } \\
\text { Points }\end{array}$ & Adjectival Rating & Weight & $\begin{array}{c}\text { Weighted } \\
\text { Score }\end{array}$ & $\begin{array}{c}\text { Overall } \\
\text { Weighted } \\
\text { Score }\end{array}$ \\
\hline $\begin{array}{l}3.2 .1 \text { Support growth of the } \\
\text { local and regional technology- } \\
\text { based primary business sector }\end{array}$ & \multicolumn{3}{|l|}{} & & \\
$\begin{array}{l}\text { 3.2.1.1 The number of new } \\
\text { business and expansions in } \\
\text { the local area where Battelle } \\
\text { had a material role in their } \\
\text { establishment }\end{array}$ & 4 & Outstanding & $30 \%$ & 1.20 & \\
$\begin{array}{l}3.2 .1 .2 \text { Effectiveness in } \\
\text { providing technical assistance } \\
\text { to local and regional firms }\end{array}$ & 4 & Outstanding & $35 \%$ & 1.40 & \\
$\begin{array}{l}\text { 3.2.1.3 Develop and champion } \\
\text { at least one new economic } \\
\text { development initiative }\end{array}$ & 4 & Outstanding & $35 \%$ & 1.40 & \\
\hline
\end{tabular}

Table A.17. Performance Indicator 3.2.2 Score Calculation

\begin{tabular}{|c|c|c|c|c|c|}
\hline Element & $\begin{array}{l}\text { Value } \\
\text { Points }\end{array}$ & Adjectival Rating & Weight & $\begin{array}{l}\text { Weighted } \\
\text { Score }\end{array}$ & $\begin{array}{c}\text { Overall } \\
\text { Weighted } \\
\text { Score }\end{array}$ \\
\hline \multicolumn{6}{|l|}{$\begin{array}{l}3.2 .2 \text { Document the success } \\
\text { of the region's major research } \\
\text { institutions in their collabora- } \\
\text { tion to find science and } \\
\text { technology solutions to } \\
\text { regional needs }\end{array}$} \\
\hline $\begin{array}{l}\text { 3.2.2.1 Find solutions to } \\
\text { significant regional needs } \\
\text { from the science and techno- } \\
\text { logy resources available in the } \\
\text { Northwest's major research } \\
\text { institutions }\end{array}$ & 4 & Outstanding & $35 \%$ & 1.40 & \\
\hline $\begin{array}{l}\text { 3.2.2.2 Determine, or cause to } \\
\text { be determined, the applicability } \\
\text { of the identified potential } \\
\text { science and technology } \\
\text { solutions and identify and/or } \\
\text { develop and utilize mecha- } \\
\text { nisms for implementing them }\end{array}$ & 4 & Outstanding & $30 \%$ & 1.20 & \\
\hline $\begin{array}{l}\text { 3.2.2.3 Demonstrate the } \\
\text { relationships established } \\
\text { among the research institu- } \\
\text { tions of the Northwest and the } \\
\text { successes of this group in } \\
\text { developing a process for and } \\
\text { finding science and technology } \\
\text { solutions to regional issues } \\
\text { and needs }\end{array}$ & 4 & Outstanding & $35 \%$ & 1.40 & \\
\hline \multicolumn{5}{|c|}{ Indicator 3.2.2Total } & 4.00 \\
\hline
\end{tabular}




\section{Appendix B History of PNNL Accomplishments}




\section{Pacific Northwest National Laboratory}

\section{A Chronology of Significant Accomplishments}

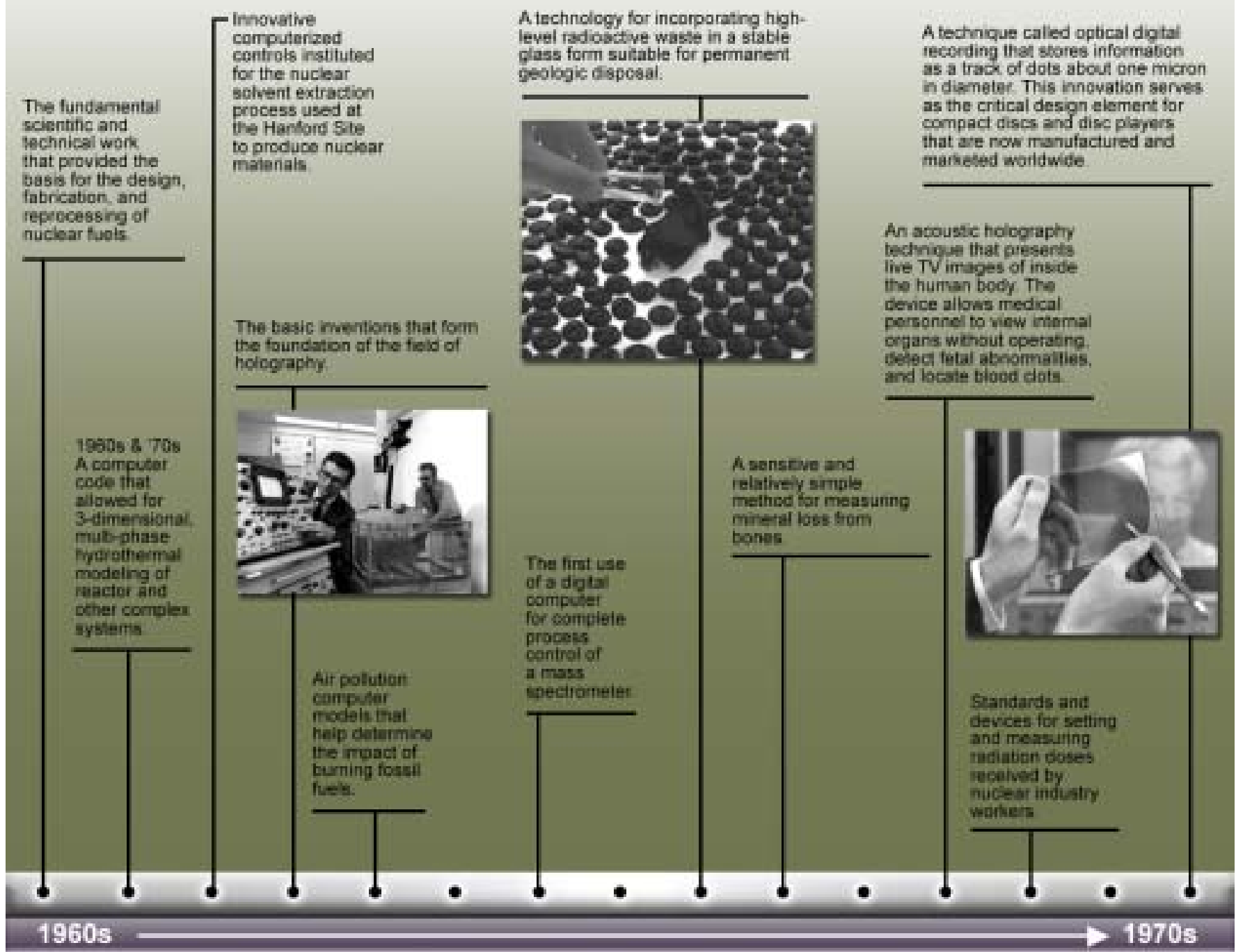




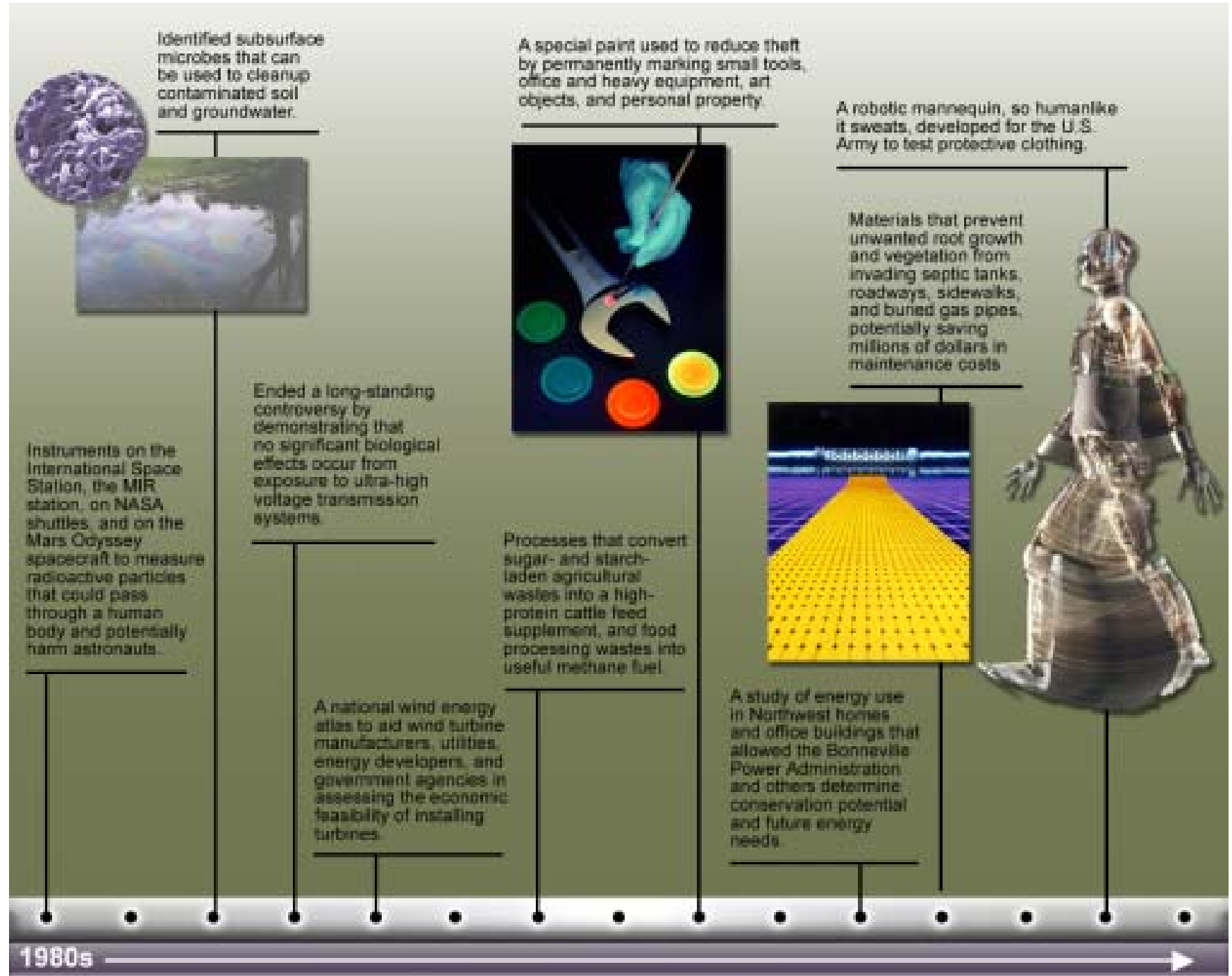




\section{Pacific Northwest National Laboratory}

\section{A Chronology of Significant Accomplishments (cont'd)}

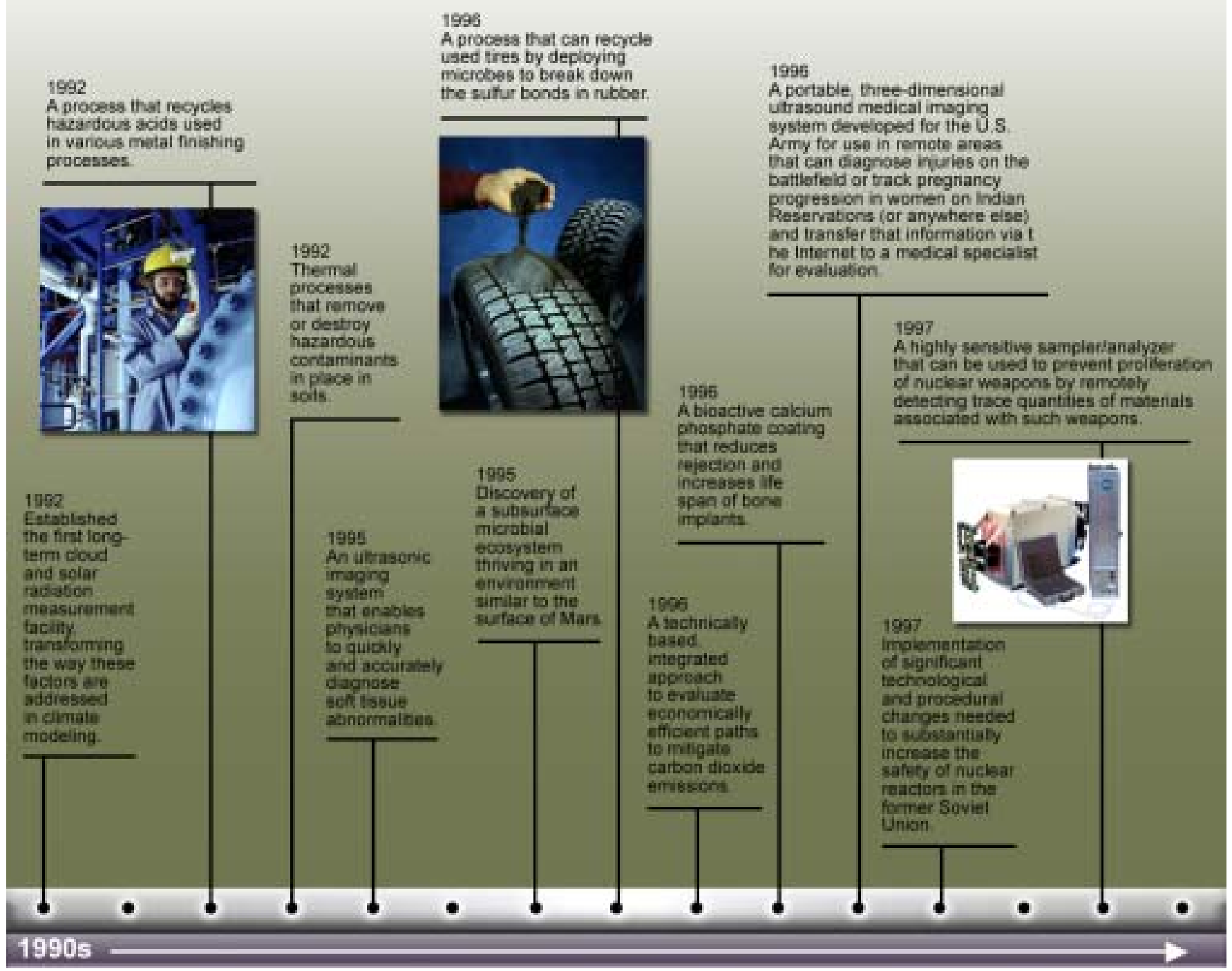




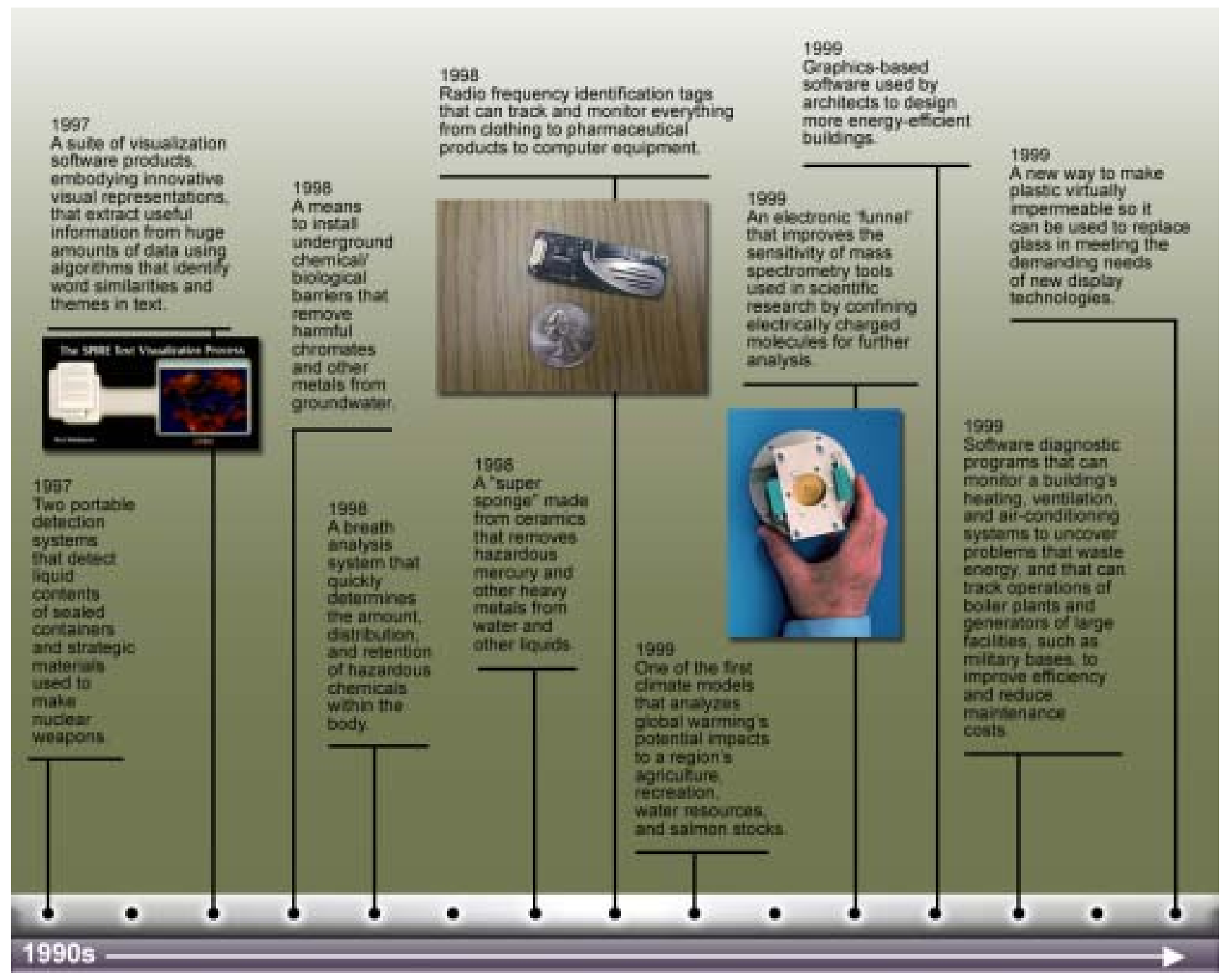




\section{Pacific Northwest National Laboratory A Chronology of Significant Accomplishments (cont'd)}

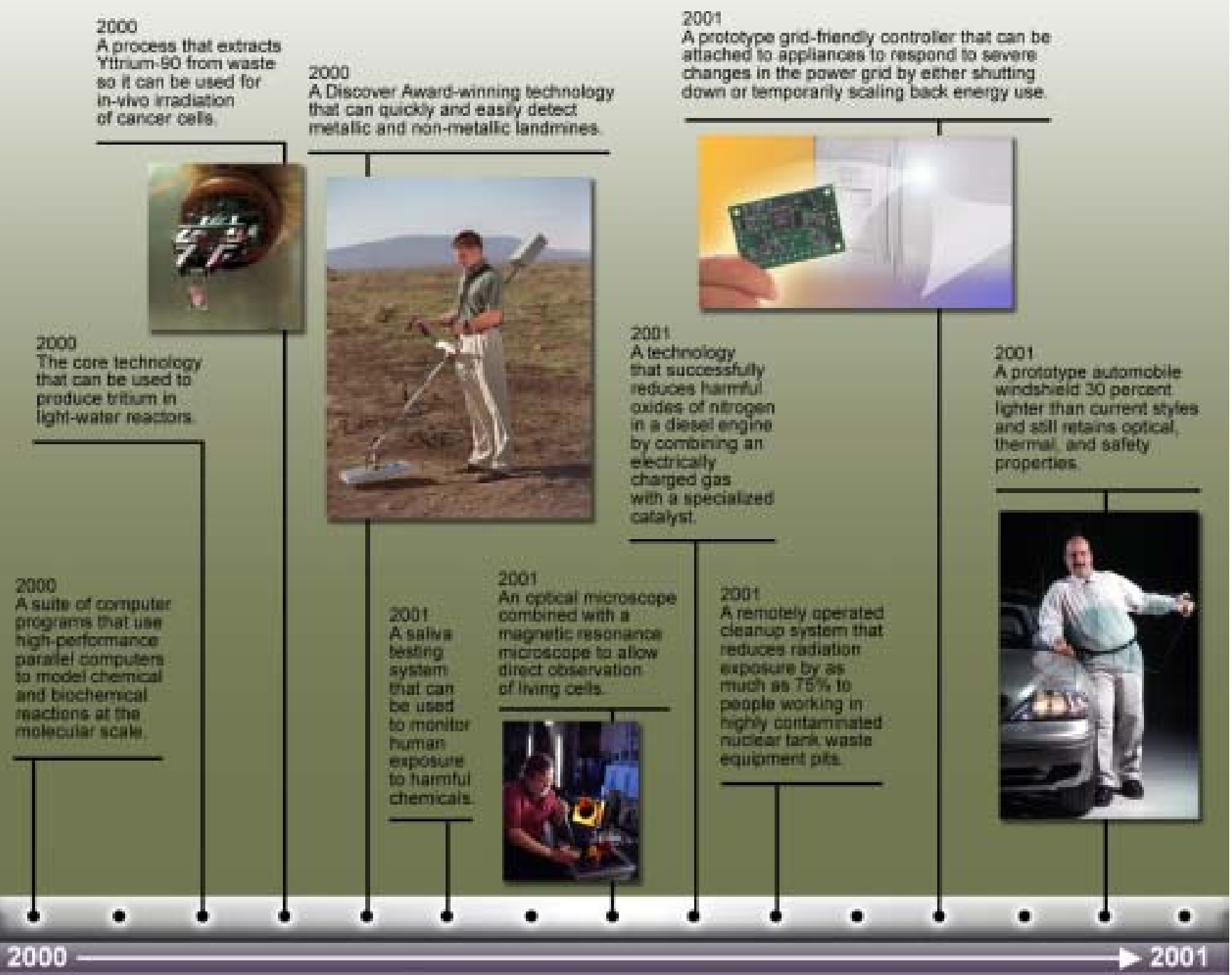




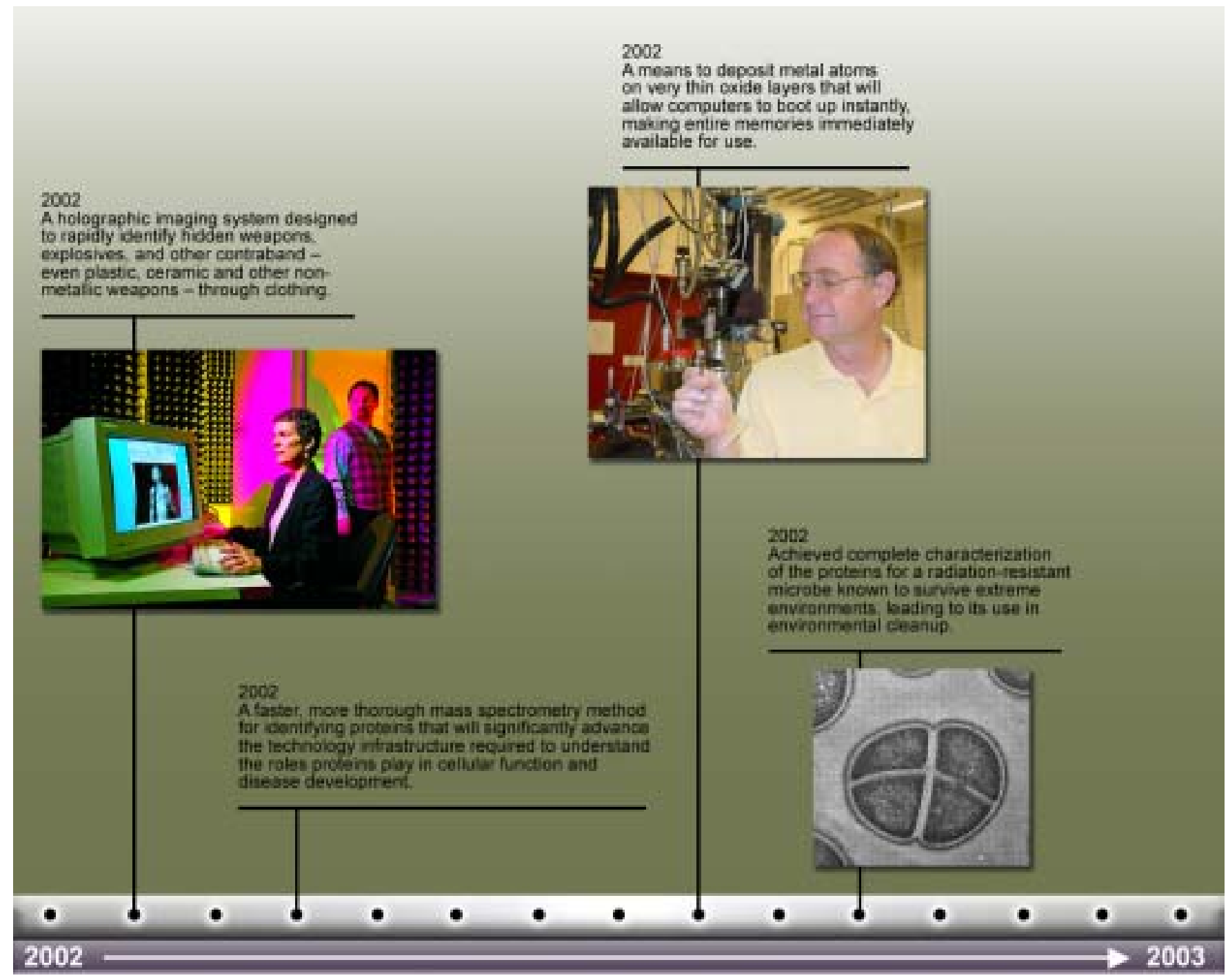





\section{Appendix C History of Significant PNNL Awards}




\section{History of Significant PNNL Awards}

Since its beginning in 1965, the Pacific Northwest National Laboratory has become a key center for science and technology in the Northwest, contributing to the region's economic development, linking its resources and ideas with universities and industries, and bringing revenues to Washington State and the Tri-Cities.

Over the past 38 years, PNNL has been honored with more than 100 awards from prestigious annual competitions sponsored by $R \& D$ Magazine, the Federal Laboratory Consortium, Discover magazine, and the U.S. Department of Energy. The Laboratory has received

- Four Discover Awards. Discover magazine annually presents Discover Magazine Innovation Awards-one in each of eight categories-to recognize the nation's top scientific and technological developments.

Landmine detector-Christopher Columbus Fellowship Foundation grant, 2001

Combined optical and magnetic resonance microscope-Health category, 2001

Micro-plasmatron fuel converter-co-winner with Massachusetts Institute of Technology in the Transportation category, 1999

Portable medical system for ultrasound imagingComputer Hardware/Electronics category, 1997

- Sixty-two R\&D 100 awards. The award, established by R\&D Magazine, honors the 100 best technical achievements each year.

\section{3}

FT-MS Proteome Express

Product Acoustic Signature System

The Starlight Information Visualization System

\section{2}

$\mathrm{OmniViz}^{\mathrm{TM}}$

\section{1}

Catalyst Materials for Plasma-Catalysis Engine Exhaust Treatment

Decision Support for Operations and Maintenance ${ }^{\mathrm{TM}}$ Long-Range Semi-Passive Radio Frequency Identification System

MilliWave Viscometer
2000

Multi-Blade Knife Failure Detector for Food Processing

Sunna Dosimeter ${ }^{\mathrm{TM}}$

Ultra Barrier Coatings for Flat Panel Displays

1999

MicroHeater

Compact Microchannel Fuel Vaporizer

PUMA Fiber Optic Neutron and Gamma Ray Sensor

Electrodynamic Ion Funnel

Molecular Sciences Software Suite (MS $\left.{ }^{3}\right)$

Centrate Ammonia Recovery Process

1998

Self-Assembled Monolayers on Mesoporous Supports MICLEAN $^{\mathrm{TM}} /$ MICARE $^{\mathrm{TM}}$ Solvent Cleaning Systems Radionuclide Aerosol Sampler/Analyzer

Rapid Dialyzer for cleansing biological samples prior to analysis.

In Situ Redox Manipulation

Life-Cycle Advantage ${ }^{\mathrm{TM}}$

R-TiC Metals Emission Monitor

1997

RubberCycle $^{\mathrm{TM}}$

Production of Chemicals from Biologically Derived Succinic Acid

Refractory Corrosion Monitor

1996

Plasma Source Quistor Mass Spectrometer

Liquid Multilayer/Polymer Multilayer Processes for Vacuum Deposition of Polymer Films

SPIRE: Spatial Paradigm for Information Retrieval and Exploration Software

Catalyzed Electrochemical Oxidation

Autonomous Environmental Sentinel 
1995

Electrical Remediation at Contaminated Environments

Real Time Ultrasonic Imaging System

Microwave Plasma Continuous Emissions Monitor

\section{4}

Ultrasonic Microstructural Analyzer

\section{3}

High-Energy Corona Reactor

Ultrafine Powder Formation by Continuous Hydrothermal Synthesis

\section{2}

Base-Catalyzed Destruction Process

Cooled, Optically Stimulated Luminescence

Glycine-Nitrate Process for Producing Ultrafine Metal

Oxide Powders

\section{1}

Planar Waveguide Spectrometer (joint entry with University of Washington)

Quantitative Luminescence Imaging System (joint entry with USAF)

Electro-Optic Liquid Sensor

Conversion of Fermentable Carbohydrates to Acrylate Esters-Lactic Acid and Lower Alkyl Acrylates Production

Petroleum Sludge Treatment Process (joint entry)

Waste Acid Detoxification and Reclamation

1990

Pyroflux Glass Melting Process

Q-Endoscreen

\section{9}

Thermochemical Environmental Energy System ${ }^{\circledR}$

\section{8}

Hydrogen Gas Recovery

Sludge-to-Oil Reactor System

Rapid Expansion of Supercritical Fluid Solutions

Electrospray Ionization Interface

\section{6}

Computer Aided Genetic Engineering/Genetic Engineering Machine

\section{4}

Noble Metals Recovery
1983

Supercritical Fluid Chromatography/Mass Spectrometer

1982

Portable Blood Irradiator

1974

Optical Digital Recording

1972

Void Metal Composite

1969

Acoustic Emission Tester

- Fifty-four Citations for Excellence in Technology Transfer from the Federal Laboratory Consortium. The FLC includes more than 700 laboratories from 16 federal agencies. FLC Awards recognize outstanding work in transferring U.S. government-sponsored technologies to the public and private sectors. PNNL has earned more FLC Awards than any other national laboratory.

\section{3}

The Acoustic Inspection Device

EMADVANTAGE: Emergency Management Capabilities to Support Multiple Users and Jurisdictions

Engine Exhaust Aftertreatment System Based on NonThermal Plasma-Assisted Catalysis

\section{2}

Molecular Beam Epitaxy for Semiconductor Wafer Development

Radio Frequency Identification Tags for Tracking and Inventory

Ultra-Barrier Coatings for Flat-Panel Displays

\section{1}

Yttrium-90 for Cancer Treatment

Radionuclide Detection Technologies

Multi-Blade Knife Failure Detector

EMSL Publisher Software

\section{0}

Plasma Enhanced Melter for Waste Conversion

Superplastic Forming for Automotive Component Manufacturing

Molecular Science Software Suite $\left(\mathrm{MS}^{3}\right)$

Reverse Micelles

Fiber-Optic Neutron and Gamma Ray Sensor 
1998

Six-Phase Soil Heating

Nuclide Navigator ${ }^{\mathrm{TM}}$

Spatial Paradigm for Information Retrieval and Exploration (SPIRE)

\section{7}

Catalyzed Electrochemical Oxidation

The P-Card Solution ${ }^{\mathrm{TM}}$

Plasma Source Quistor Mass Spectrometer

MECcheck ${ }^{\mathrm{TM}}$ Toolkit

\section{6}

Vacuum Process for Manufacturing Lithium Polymer Batteries

Software for Energy-Efficient Design

Automated Tool Profile Grinding Machine

\section{5}

Ultrasonic Microstructural Analyzer

Cooled, Optically Stimulated Luminescence

\section{4}

Estimates of the Wind Resources

Capillary Zone Electrophoresis/Mass Spectrometry

Fast, Adaptive Communications Software

\section{3}

Waste Acid Recovery Systems

$\mathrm{ReOpt}^{\mathrm{TM}}$ Software

Glycine-Nitrate Process produces Ultrafine Metal

Oxide Powders

MEPAS Software

\section{2}

Electro-Optic Liquid Sensor

Portable Blood Irradiator for Treating Blood Diseases TEMPEST Code for Hydrothermal Analysis

\section{1}

Survey Meter Recorder

Ceramic Melter Technology

In Situ Vitrification

\section{0}

Steel Temperature-Measuring Device

Conversion of Fermentable Carbohydrates to Acrylate Esters-Lactic Acid and Lower Alkyl Acrylaters Production

\section{9}

Thermochemical Environmental Energy System ${ }^{\circledR}$

Computer Aided Genetic Engineering/Genetic Engineering Machine
1988

Sludge-to-Oil Conversion

1987

Kinetic Phosphorimetry Technique

Low-Cost Sampling Pump

\section{6}

Biobarrier Technology

Synthetic Aperture Focusing Technology

\section{5}

Advanced Decontamination Techniques

Dry Cooling Enhancement Program

\section{4}

Neutron Dosimetry Technology

Thermal Hydraulics for Reactors

- Six Energy 100 awards. The Department of Energy awarded 100 discoveries and innovation that have resulted in improvements for American consumers (1977-2000).

1999 - ASHRAE/IESNA Standard 90.1 - 1999. Energy Efficiency for Buildings Except Low Rise Residential

1997 - Biomimetic Coatings for Orthopedic Implants

1995 - Wide Area Measurement System.

1994 - MECcheck: Making Sense of the Model Energy Code.

1990 - Production of the Medical Isotope Yttrium-90 from the Fission Byproduct Strontium-90.

1988 - An Improved Method of Biological Sample Analysis: Capillary Zone Electrophoresis - Mass Spectrometry

PNNL staff have earned a variety of awards during the past four decades. Selected recent awards include

- Five E.O. Lawrence Memorial Awards. The awards are presented to scientists and engineers under age 45 "for especially meritorious contributions" in nuclear research. Those honored were:

Thom Dunning, 1996

Raymond E. Wildung, 1982

Edwin D. McClanahan Jr., 1976

Charles E. Elderkin, 1975

William J. Bair, 1970 
- One DOE Office of Science Young Scientist Award and one Presidential Early Career Science and Engineering (PECASE) Award.

Mari-Lou Balmer, 1994

Other Laboratory highlights for the past four decades include

- Receiving 1043 patents (653 U.S. and 390 foreign) on inventions by staff members. The first patent, issued to Lane Bray in 1967, was for Zirconium removal from Strontium-90.

- Using PNNL technology, executive-level staff, and/or equity investment to form the basis for about 100 businesses nationwide. More than twothirds of them were established in Washington, with about 80 percent of those in the Tri-Cities. More than 40 of these companies used technology developed at PNNL as the basis for a new product. Since 1996, more than 550 businesses - about 90 percent of them in the Northwest - have used PNNL's technical and entrepreneurial assistance to get started or establish new product lines.

- Distributing more than $\$ 11$ million to charitable interests.

- Volunteering over 60,200 hours in the community in the last five years. 

Appendix D
Acronyms 


\section{Appendix D - Acronyms}

\begin{tabular}{|c|c|}
\hline ADM & Archer Daniels Midland Corporation \\
\hline ALD & Associate Laboratory Director \\
\hline AMT & Associate Manager for Science and Technology \\
\hline AMT & Accurate Mass and Time \\
\hline ANL & Argonne National Laboratory \\
\hline ANSTI & Advanced Nuclear Science and Technology \\
\hline APU & Auxiliary Power Units \\
\hline ARM & Atmospheric Radiation Monitoring program \\
\hline ASAT & All the Signal, All the Time \\
\hline ASCR & Advanced Scientific Computing Research \\
\hline ASME & American Society of Mechanical Engineers \\
\hline ASO & Analytical Support Operations \\
\hline BBPI & Bio-Based Products Initiative \\
\hline BECP & Building Energy Codes Project \\
\hline BER & U.S. Department of Energy, Office of Biological and Environmental Research \\
\hline BERAC & BER Advisory Committee \\
\hline BES & U.S. Department of Energy, Office of Basic Energy Science \\
\hline BGC & Biology Grand Challenge \\
\hline BGGC & BioGeochemistry Grand Challenge \\
\hline BMI & Battelle Memorial Institute \\
\hline BSI & Biomolecular Systems Initiative \\
\hline BSS & Business Support Services \\
\hline CAT & Collaborative Access Team \\
\hline $\mathrm{CBC}$ & Columbia Basin College \\
\hline CBP & Bureau of Customs and Border Protection \\
\hline $\mathrm{CD}$ & Critical Decision \\
\hline CDFA & Capability Development Focus Area \\
\hline CEO & Chief Executive Officer \\
\hline $\mathrm{CHG}$ & CH2M HILL \\
\hline CI & PNNL Counterintelligence \\
\hline CIA & Central Intelligence Agency \\
\hline CIDI & Compression Injection Direct Injection \\
\hline $\mathrm{CIO}$ & Counterintelligence Office \\
\hline $\mathrm{CN}$ & U.S. Department of Energy, Office of Counterintelligence \\
\hline COMSEC & Communications Security \\
\hline COTS & Commercial Off The Shelf \\
\hline CPR & Cost Productivity Review \\
\hline $\mathrm{CR}$ & Continuing Resolution \\
\hline CRADA & Cooperative Research and Development Agreement \\
\hline CS\&E & Computational Science \& Engineering \\
\hline CS\&EI & Computational Science \& Engineering Initiative \\
\hline CSM & Customer Service Model \\
\hline CSNF & Commercial Spent Nuclear Fuel \\
\hline CTP & Core Technology Program \\
\hline
\end{tabular}




\begin{tabular}{|c|c|}
\hline DARPA & Defense Advanced Research Projects Agency \\
\hline DART & Days Away, Restricted of Transferred \\
\hline $\mathrm{DC}$ & Direct Current \\
\hline $\mathrm{D} \& \mathrm{D}$ & Decontamination and Decommissioning \\
\hline DEA & Drug Enforcement Agency \\
\hline DHS & Department of Homeland Security \\
\hline DNAPL & Dense Non-Aqueous Phase Liquids \\
\hline DoD & Department of Defense \\
\hline DOE & U.S. Department of Energy \\
\hline DOELAP & Department of Energy Laboratory Accreditation Program \\
\hline DOE-CN & U.S. Department of Energy, Office of Counterintelligence \\
\hline DOE-EH & U.S. Department of Energy, Office of Health, Environment and Safety \\
\hline DOE-EM & U.S. Department of Energy, Office of Environmental Management \\
\hline DOE-FE & U.S. Department of Energy, Office of Fossil Energy \\
\hline DOE-HQ & U.S. Department of Energy, Headquarters \\
\hline DOE-IN & U.S. Department of Energy, Office of Intelligence \\
\hline DOE-NA-22 & U.S. Department of Energy, Office on Nonproliferation Research and Engineering \\
\hline DOE-NABIR & U.S. Department of Energy, Natural and Accelerated Bioremediation \\
\hline DOE-NE & U.S. Department of Energy, Office of Nuclear Energy \\
\hline DOE-NE-50 & U.S. Department of Energy, Office of Space and Defense Power Systems \\
\hline DOE-OBER & U.S. Department of Energy, Office of Biological and Environmental Research \\
\hline DOE-ORP & U.S. Department of Energy, Office of River Protection \\
\hline DOE-RL & U.S. Department of Energy, Richland Operations \\
\hline DOE-RW & U.S. Department of Energy, Office of Civilian Radioactive Waste Management \\
\hline DOE-SC & U.S. Department of Energy, Office of Science \\
\hline DRC & Directorate Review Committee \\
\hline DRP & Disaster Recovery Program \\
\hline DST & Hanford Double-Shell Tank \\
\hline EDO & Economic Development Office \\
\hline EERE & U.S. Department of Energy, Office of Energy Efficiency and Renewable Energy \\
\hline EEOICPA & Energy Employee Occupational Illness Compensation Program Act \\
\hline EIS & Environmental Impact Statement \\
\hline EMSL & Environmental Molecular Science Laboratory \\
\hline EMS & Environmental Management Systems \\
\hline EMSP & Environmental Management Science Program \\
\hline EPA & Environmental Protection Agency \\
\hline EPR & Electronic Prep \& Risk \\
\hline EPR/ESR & Electron Paramagnetic Resonance/Electron Spin Resonance \\
\hline EPS & Essential Power System \\
\hline ERICA & Electronic Records and Information Capture Architecture \\
\hline ERS & EMSL Resource System \\
\hline ES\&H & Environment Safety \& Health \\
\hline ESH\&Q & Environment, Safety, Health and Quality \\
\hline ESnet & Energy Sciences Network \\
\hline ESTD & Energy Science \& Technology Directorate \\
\hline ETD & Environmental Technology Directorate \\
\hline EUS & EMSL User System \\
\hline
\end{tabular}




\begin{tabular}{|c|c|}
\hline Fab & Functional antigen-binding fragment \\
\hline $\mathrm{FE}$ & U.S. Department of Energy, Office of Fossil Energy \\
\hline FEMIS & Federal Emergency Management Information System \\
\hline FH & Fluor Hanford \\
\hline FIR & Field Investigation Report \\
\hline FLC & Federal Laboratory Consortium \\
\hline $\mathrm{F} \& \mathrm{O}$ & Facilities and Operations \\
\hline FSD & Fundamental Sciences Directorate \\
\hline FTE & Full Time Equivalent \\
\hline FTICR & Fourier Transform Ion Cyclotron Resonance \\
\hline FTIR & Fourier Transform Infrared Spectroscopy \\
\hline FT-MS & Fourier Transform ion cyclotron Mass Spectrometer \\
\hline FUA & Facility Use Agreements \\
\hline GAN & Gosatomnadzor - Russian regulatory agency \\
\hline GB & Gigabyte \\
\hline GE & General Electric \\
\hline $\mathrm{GHz}$ & Gigahertz \\
\hline GTL & Genomes to Life \\
\hline GTLA & Government Technology Leadership Awards \\
\hline $\mathrm{H} \& \mathrm{~S}$ & Health and Safety \\
\hline HAMMER & Hazardous Materials Management and Emergency Respons \\
\hline HCMV & Human Cytomegalovirus \\
\hline HEU & Highly Enriched Uranium \\
\hline HiTEC & High Temperature Electrochemistry Center \\
\hline HP & Hewlett-Packard \\
\hline HQ & U.S. Department of Energy, Headquarters \\
\hline $\mathrm{HS}$ & Homeland Security \\
\hline HSW & Hanford Solid Waste \\
\hline IA & Internal Auditing \\
\hline IC & Intelligence Community \\
\hline ICCP & Information Classification and Control Policy \\
\hline IFMA & International Facility Management Association \\
\hline IIR & Information Intelligent Reports \\
\hline IMAC & Inquiry Management and Analysis Capability \\
\hline IN & U.S. Department of Energy, Office of Intelligence \\
\hline INEEL & Idaho National Engineering and Environmental Laboratory \\
\hline I-NERI & International Nuclear Research Initiative \\
\hline INS & Integrated Nuclear Strategy \\
\hline IO & Independent Oversight \\
\hline IOPS & Integrated Operations System \\
\hline IP & Intellectual Property \\
\hline IPA & Interdepartmental Personnel Assignments \\
\hline ISA & In-depth Safety Assessment \\
\hline ISI & Institute for Scientific Information \\
\hline ISMS & Integrated Safety Management System \\
\hline ISO & International Organization for Standardization \\
\hline ISSM & Integrated Safeguards and Security Management \\
\hline IT & Information Technology \\
\hline IWFO & Intelligence Work for Others \\
\hline
\end{tabular}


JIMO Jupiter Icy Moons Orbiter

$\begin{array}{ll}\text { LA } & \text { License Application } \\ \text { LASER } & \text { Leadership and Assistance for Science Education Reform } \\ \text { LAW } & \text { Low Activity Waste } \\ \text { LC } & \text { Liquid Chromatography } \\ \text { LDRD } & \text { Laboratory Directed Research and Development } \\ \text { LRR } & \text { Linking Regional Resources } \\ \text { LSL } & \text { Life Science Laboratory } \\ \text { LWR } & \text { Light Water Reactor } \\ & \\ \text { MHz } & \text { Megahertz } \\ \text { MIT } & \text { Massachusetts Institute of Technology } \\ \text { M\&O } & \text { Managing and Operating } \\ \text { MPI } & \text { Message Passing Interface } \\ \text { MPS } & \text { Minimum Performance Standards } \\ \text { MS } & \text { Mass Spectrometry } \\ \text { MSO } & \text { Management System Owner } \\ \text { MSU } & \text { Montana State University } \\ \text { MYPP } & \text { Multi-Year Program Plan }\end{array}$

NA-20 U.S. Department of Energy, Office of Defense Nuclear Nonproliferation

NA-22

NA-23

NA-24

NA-25

NA-26

NABIR

NAF

NAPL

NASA

NBC

NBC

NCRR

NDA

NDE

NE

NE-50

NEPA

Office on Nonproliferation Research and Engineering

Office of International Nuclear Safety and Cooperation

Office of Nonproliferation and International Security

Office of International Material Production and Cooperation

Office of Fissile Materials Disposition

Natural and Accelerated Bioremediation

Nipple Aspirate Fluid

Nonaqueous Phase Liquid

National Aeronautics and Space Administration

Nuclear Biological Chemical

National Bioenergy Center

National Center for Research Resources

Non-Destructive Analyses

Non-Destructive Examination

U.S. Department of Energy, Office of Nuclear Energy

NERAC Nuclear Energy Research Advisory Committee

NERI Nuclear Energy Research Initiative

NETL National Energy Technology Laboratory

NIC

$\mathrm{NIH}$

National Intelligence Council

National Institute of Health

NIOSH

NITRD

NNSA

NMR

National Institute for Occupational Safety and Health

National Coordination Office for Information Technology Research and Development

National Nuclear Security Administration

National Magnetic Resonance

NORM

Naturally Occurring Radioactive Material 


\begin{tabular}{|c|c|}
\hline $\mathrm{NO}_{x}$ & Nitrogen Oxides \\
\hline NREL & National Renewable Energy Laboratory \\
\hline NRC & Nuclear Regulatory Commission \\
\hline NSD & National Security Directorate \\
\hline NSTI & Nanoscience \& Technology Initiative \\
\hline NTP & Non Thermal Plasma \\
\hline NWECT & Northwest Energy Technology Collaborative \\
\hline $\mathrm{OA}$ & Independent Oversight and Performance Assurance \\
\hline $\mathrm{OAC}$ & Operational Analysis Center \\
\hline OBER & U.S. Department of Energy, Office of Biological and Environmental Research \\
\hline OBP & Office of the Business Program \\
\hline OBP & Office of Biomass Programs \\
\hline $\mathrm{OC}$ & Optical Carrier \\
\hline OETD & Office of Electricity Transmission and Distribution \\
\hline OFCVT & Office of Freedom Car and Vehicle Technologies \\
\hline OHSU & Oregon Health Sciences University \\
\hline OMB & Office of Management and Budget \\
\hline ORNL & Oakridge National Laboratory \\
\hline ORPS & Occurrence Reporting and Processing System \\
\hline OSL & Optically Stimulated Luminescence \\
\hline OSU & Oregon State University \\
\hline PASS & Product Acoustic Signature System \\
\hline PDA & Personal Digital Assistant \\
\hline PEFA & Performance Evaluation and Fee Agreement \\
\hline PFP & Plutonium Finishing Plant \\
\hline PI & Principal Investigator \\
\hline PiCEIS & Parallel Computational Environment for Imaging Science \\
\hline PM & Particulate Matter \\
\hline PNNL & Pacific Northwest National Laboratory \\
\hline PNWGP & Pacific Northwest Gigapop \\
\hline PNWRC & Pacific Northwest Regional Collaboratory \\
\hline PNSO & Pacific Northwest Site Office \\
\hline PROX & Preferential Oxidation Reactor \\
\hline RAM & Random Access Memory \\
\hline $\mathrm{R} \& \mathrm{D}$ & Research and Development \\
\hline RBAC & Role Based Access Control \\
\hline $\mathrm{RCP}$ & Regional Carbon Partnership \\
\hline RCSP & Remediation and Closure Science Project \\
\hline RDAC & Radiation Detection Analysis Center \\
\hline RHEED & Reflection High Energy Electron Diffraction \\
\hline RICC & Residential IECC Code Change \\
\hline RL & U.S. Department of Energy, Richland Operations \\
\hline ROD & Record of Decision \\
\hline RONDE & Remotely Operated Nondestructive Evaluation \\
\hline RPL & Radiochemical Processing Laboratory \\
\hline RPM & Radiation Portal Monitor \\
\hline RW & U.S. Department of Energy, Office of Civilian Radioactive Waste Manag \\
\hline
\end{tabular}




\begin{tabular}{|c|c|}
\hline S\&T & Science and Technology \\
\hline SAMMS & Self-Assembled Monolayers on Mesoporous Supports \\
\hline SAS & Safeguards and Security \\
\hline SASS & Safegaurds and Security Services \\
\hline SC & U.S. Department of Energy, Office of Science \\
\hline SCADA & Supervisory Control and Data Acquisition \\
\hline SCIF & Sensitive Compartmentalized Information Facility \\
\hline SHEBA & Surface Heat Budget of the Arctic Ocean \\
\hline SECA & Solid Energy Conversion Alliance \\
\hline SEN & Single Enzyme Nanoparticle \\
\hline SEM & Scanning Electron Microscope \\
\hline SGI & Silicon Graphics Inc. \\
\hline SOFC & Solid Oxide Fuel Cells \\
\hline STOMP & Subsurface Transport over Multiple Phases \\
\hline SW-EIS & Solid Waste Environmental Impact Statement \\
\hline SWOT & Strengths, Weaknesses, Opportunities, Threats \\
\hline TB & Terabytes \\
\hline Tc & Technetium \\
\hline TEM & Transmission Electron Microscope \\
\hline TEMPEST & Emissions Security \\
\hline TRCR & Total Recordable Case Rate \\
\hline TRLINX & Three Rivers Internet Exchange \\
\hline TRU & Transuranic \\
\hline TTIC & Terrorist Threat Integration Center \\
\hline USCAR & United States Council for Automotive Research \\
\hline USDA & United States Department of Agriculture \\
\hline USSOCOM & United States Special Operations Command \\
\hline UT & University of Texas \\
\hline UW & University of Washington \\
\hline VPP & Voluntary Protection Program \\
\hline WASL & Washington Assessment for Student Learning \\
\hline WEA & Workplace Engagement Assessment \\
\hline WISHA & Washington Industrial Safety and Health Act \\
\hline WMD & Weapons of Mass Destruction \\
\hline WSSX & Warhead Safety and Security Exchange \\
\hline WSU & Washington State University \\
\hline WSU-TC & Washington State University - Tri-Cities \\
\hline ZERT & Zero Emissions Research and Technology \\
\hline
\end{tabular}


